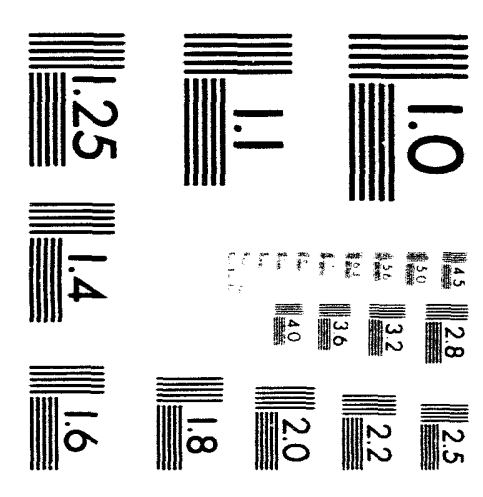



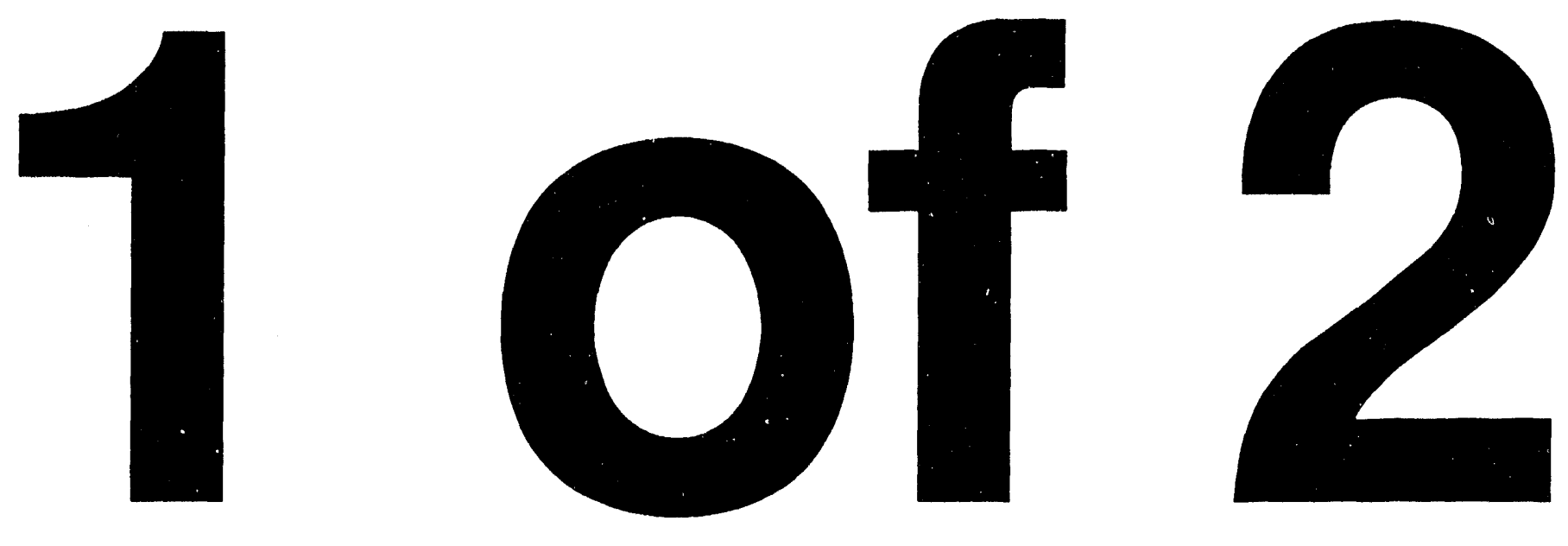


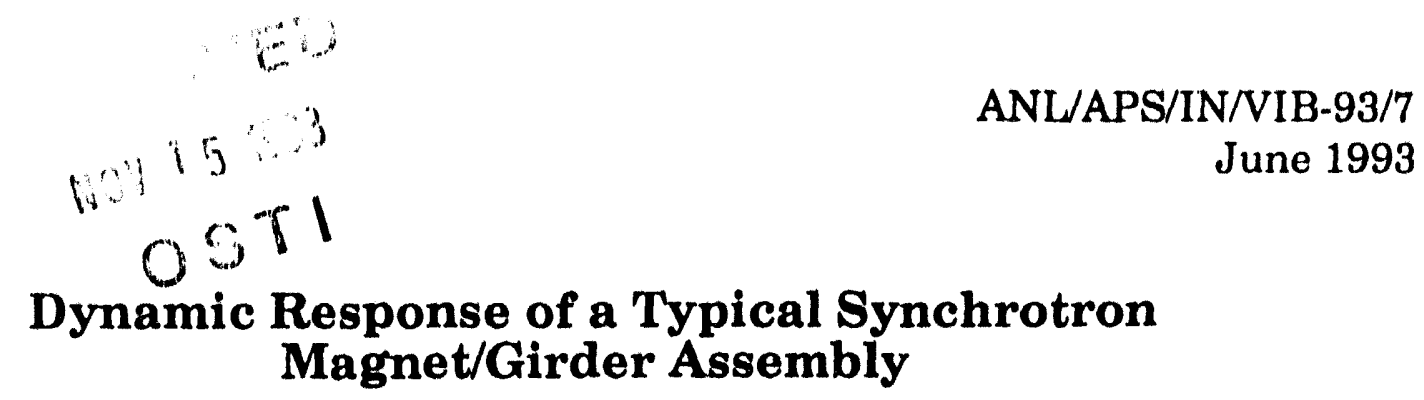

by

\author{
J. A. Jendrzejczyk, R. K. Smith, and M. E. Vogt \\ Argonne National Laboratory
}

\title{
Introduction
}

In the Advanced Photon Source, the synchrotron booster ring accelerates positrons to the required energy level of $7 \mathrm{GeV}$. The positrons are then injected into the storage ring where they continue to orbit for 10-15 h. The storage ring quadrupoles have very stringent vibration criteria that must be satisfied to ensure that beam emittance growth is within acceptable limits, viz., $<10 \%$. Because the synchrotron booster ring is not operated after particle insertion into the storage ring, its vibration response is not a critical issue relative to the performance of the storage ring beam. Nevertheless, the synchrotron pulses at a frequency of $2 \mathrm{~Hz}$, and if a vibration response frequency of the synchrotron magnet/girder assembly were to coincide with the pulsation frequency or its near harmonics, large-amplitude motion could result, with the effect that it could compromise the operation of the synchrotron. Due to the complex dynamics of the synchrotron magnet/girder assembly, it is necessary to measure the dynamic response of a prototypic assembly and its components to ensure that the inherent dynamic response frequencies are not equal to $2 \mathrm{~Hz}$ or any near harmonics. Dynamic-response measurement of the synchrotron girder assembly and component magnets is the subject of this report.

\section{Technical Approach}

The main objective of this study is to measure the dynamic response frequencies of the major component magnets on the synchrotron girder assembly and the transfer functions of the response at $2 \mathrm{~Hz}$ or any close harmonics. To accomplish this, a small electrodynamic exciter was mounted to the top surface of the girder, and, in turn, oriented to excite motion in three different directions: $\mathrm{X}$ horizontal, transverse to the beam; Y - vertical; and $\mathrm{Z}$ - horizontal in the beam direction. The exciter shown in Fig. 1 is oriented to provide force excitation in the $\mathrm{X}$ direction. A signal generator and amplifier were used to control and provide power

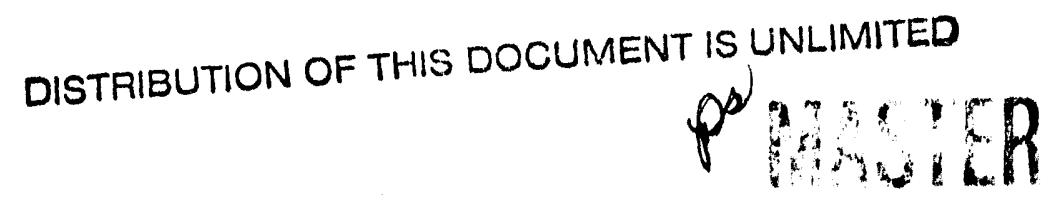


to drive the exciter. Typically, the force excitation range was $1-100 \mathrm{~Hz}$ random force at $<1 \mathrm{lb}$ total RMS. If a large low-frequency response were to be observed, sinusoidal force excitation can be provided at that specific frequency to measure transfer functions. Impulse excitation, provided by a slight tap with a finger or hand, was also used to determine response frequencies of specific components.

The low-frequency response of the girder assembly is controlled by the large massive components, that is, the magnets. A series of triaxial (acceleration measurements in three axes, $\mathrm{X}, \mathrm{Y}$, and $\mathrm{Z}$ ) dynamic response measurements was made on each of the four magnets: the bending magnet, the horizontal corrector, the quadrupole, and the sextupole. Simultaneous measurements were also made at a specific location on the girder. A single accelerometer oriented vertically ( $Y$ direction) was mounted to the floor near the center leg of the girder. A typical measurement setup, showing one triaxial accelerometer group on the girder and the other on the sextupole magnet, is shown in Fig. 1.

To obtain the required low-frequency sensitivity, acceleration must be used because typical analog double-integration techniques would limit the low-frequency response of the measurement system. Small PCB 336 B accelerometers were used to measure acceleration; the small size ensures that the mass of the measuring devices will not affect the system dynamics. The acceleration signals were amplified and recorded on an FM tape recorder. The signals were then analyzed for spectral content with a Zonic FFT analyzer. To get an indication of system response amplitudes, the acceleration signals were double integrated to obtain displacement and used to approximate transfer functions.

In conducting the measurement test series, a specific magnet was instrumented as described above. The exciter was positioned in three orthogonal directions with reference to the positron beam. Acceleration data from all seven accelerometers was recorded for each exciter position for a period of $\approx 5 \mathrm{~min}$. This process was continued until the dynamic responses of all the magnets were measured.

\section{Discussion}

\section{Bending (dipole) magnet/girder assembly}

A schematic representation of the bending-magnet/girder assembly that describes the measurement locations and directions is shown in Fig. 2. A series of power spectral density (PSD) plots was generated for the three excitation directions; see Figs. A1-28 of Appendix A. Although dynamic responses were observed at specific frequencies, none appeared to be excessively large. A compilation of the 
response frequencies is given in Table 1 . Measured damping factors were $0.7 \%$ and $0.5 \%$ in the $\mathrm{X}$ and $\mathrm{Y}$ directions, respectively.

\section{Horizontal corrector-magnet/girder assembly}

A schematic representation of the horizontal corrector-magnet/girder assembly that describes the measurement locations and directions is shown in Fig. 3. A series of PSD plots was generated for the three excitation directions; see Figs. B1-28 of Appendix B. A compilation of the response frequencies is given in Table 2. Several fairly large frequency response peaks were observed at 36,40 , and $57 \mathrm{~Hz}$. These frequencies are considerably above the frequency range of concern. However, impact excitation indicated a large response at $12 \mathrm{~Hz}$ that was not observed with random excitation to the girder, possibly the result of the system mode shapes relative to the sensor location. Measured damping factors were $\approx 0.75 \%$ for all three directions.

\section{Quadrupole magnet/girder assembly}

A schematic representation of the horizontal corrector-magnet/girder assembly that describes the measurement locations and directions is shown in Fig. 4. A series of PSD plots was generated for the three excitation directions; see Figs. C1-28 of Appendix C. A compilation of the response frequencies is given in Table 3. The primary frequency response peaks were observed at $17.9,19,21$, and $54 \mathrm{~Hz}$, which, with the exception of the $17.9 \mathrm{~Hz}$ peak, correspond to the set of frequencies obtained with impact excitation. Measured damping factors were $0.6 \%$ and $1.2 \%$ for the $\mathrm{X}$ and $\mathrm{Z}$ directions, respectively.

\section{Sextupole-magnet/girder assembly}

A schematic representation of the horizontal corrector-magnet/girder assembly that describes the measurement locations and directions is shown in Fig. 5. A series of PSD plots was generated for the three excitation directions; see Figs. D1-28 of Appendix D. A compilation of the response frequencies is given in Table 4. The primary response peaks were observed at 35,43 , and $89 \mathrm{~Hz}$, which correspond to the frequencies observed with impact excitation. Because of a large coupled-mode effect, the damping-factor values, which could only be approximated, were $\approx 1 \%$, $0.9 \%$, and $0.8 \%$ in the $\mathrm{X}, \mathrm{Y}$, and $\mathrm{Z}$ directions, respectively.

\section{Impact excitation tests}

Impact excitation is useful in determining the response frequencies of components; however, in a mechanical system as complex as the synchrotron magnet/girder assembly, coupling between components can cause confusion in 
accurately determining frequencies. If the response amplitude and mode shapes are considered, a reasonable frequency determination can be made. A compilation of the observed frequencies is given in Table 5.

\section{Girder/floor coupling}

A series of measurements was made to determine the coupling between the girder and floor. One triaxial accelerometer unit was mounted to the floor adjacent to the single vertical accelerometer and the other triaxial accelerometer unit remained on the girder, near the exciter, in the same position as when the magnet responses were measured. Because of time constraints, random excitation was only in the $\mathrm{X}$ direction, which appeared to be the most "flexible" during the previous testing. The PSD plots are given in Figs. E1-7 of Appendix E. At frequencies $>10 \mathrm{~Hz}$, there appeared to be very little coupling between the girder assembly and the floor. The majority of the frequencies $<10 \mathrm{~Hz}$ are present on both the floor and the girder assembly, indicating that they are due to floor motion and are coupled to the girder assembly.

\section{RMS displacements}

The acceleration signals were double integrated with a Zonic FFT analyzer to obtain RMS displacements within the frequency range of $1-100 \mathrm{~Hz}$. The resultant displacements are given in Table 6. The measured displacements consist of a summation of those produced by the exciter and those due to ambient ground motion. Because the exciter force was small (to avoid system nonlinearities), the resultant displacements were also small and the values shown represent a summation of both the exciter-generated and ambient displacements. Considerable activity was associated with personnel working in the area of the synchrotron girder, which, at times, resulted in a large ambient displacement. An example can be seen in the displacement level on the floor during the sextupole magnet measurement in the Y-excitation direction. Therefore, Table 6 should be used as a guide, to provide a superficial look at the system displacement, rather than as absolute response data.

\section{Comments/Conclusions}

Basic system response frequencies, obtained from Tables 1-5, are summarized in Table 7, where they are associated with their most probable source. The frequencies below $1.2 \mathrm{~Hz}$ are the result of ambient floor vibration coupling to the girder assembly. From the measurement data, the following conclusions can be drawn: 
- No large frequency responses were observed at $<12 \mathrm{~Hz}$.

- All observed frequencies below $12 \mathrm{~Hz}$ appear to be the result of ambient floor vibration

- Although measured damping was low, no large transfer function was observed.

In conclusion, the design of the synchrotron magnet support is shown to be satisfactory from the standpoint of not possessing inherent dynamic response frequencies that would have a potential for coupling with the $2-\mathrm{Hz}$ synchrotron pulsation frequency or near harmonics. 
Table 1. Frequencies $(\mathrm{Hz})$ at which peaks were measured for bending (dipole)-magnet/girder assembly under various conditions

\begin{tabular}{|c|c|c|c|c|c|c|c|c|c|c|c|c|c|c|c|c|c|c|c|c|}
\hline $\begin{array}{l}\text { Exc. } \\
\text { Dir. }^{\mathrm{a}}\end{array}$ & $\begin{array}{c}\text { Meas. } \\
\text { Dir. and } \\
\text { Location }\end{array}$ & & & & & & & & & $\begin{array}{l}\text { quenc } \\
(\mathrm{Hz})\end{array}$ & & & & & & & & & & \\
\hline$x$ & $x, m$ & 2.75 & 5.6 & & 8.5 & 11.2 & 14.1 & 17 & 18.9 & & & & 31 & & 35.9 & & & 57.9 & & \\
\hline$x$ & $\mathrm{y}, \mathrm{m}$ & 2.75 & 5.6 & & 8.5 & 11.2 & 14.1 & & & & & & 31 & 33.5 & 35.9 & 38.1 & & 57.9 & & \\
\hline$x$ & $\mathrm{z}, \mathrm{m}$ & 2.75 & 5.6 & & 8.5 & 11.2 & 14.1 & & & & & & 31 & 33.5 & & & & & & \\
\hline $\mathbf{x}$ & $x, g$ & 2.75 & 5.6 & & 8.5 & 11.2 & 13.8 & 17.5 & & & & & & 33.5 & & & 39.8 & 57.7 & 61.1 & 87.3 \\
\hline $\mathbf{x}$ & $y, g$ & 2.75 & & & 8.5 & 11.2 & & & & & & & & & & & & & & 87.3 \\
\hline$x$ & $\mathrm{z}, \mathrm{g}$ & 2.75 & & & 8.5 & 11.2 & & & & & & & & & & & & & 70 & 84.8 \\
\hline$x$ & $y, f l r$ & 3.2 & & 6.4 & 8.5 & & & & & & & & & & & & & & & 87.2 \\
\hline $\mathbf{y}$ & $\mathrm{x}, \mathrm{m}$ & 3.2 & & & 8.5 & 11.4 & 14.8 & & & & & & & & & & & & & \\
\hline $\mathbf{y}$ & $\mathrm{y}, \mathrm{m}$ & 3.2 & & & 8.5 & 11.4 & 14.8 & 17.9 & & & & & & & & & & & & \\
\hline$y$ & $\mathrm{z}, \mathrm{m}$ & 3.2 & & & 8.5 & 11.4 & 14.8 & 17.9 & & & & & & & & & & & & \\
\hline $\mathbf{y}$ & $x, g$ & 3.2 & & & 8.5 & 11.4 & 14.8 & & & & & 30.1 & & & & & & & & \\
\hline$y$ & $y, g$ & 3.2 & & & 8.5 & 11.4 & 14.8 & 17.9 & & & & & & & & & & & & \\
\hline $\mathbf{y}$ & $\mathrm{z}, \mathrm{g}$ & 3.2 & & & 8.5 & 11.4 & 14.8 & 17.9 & & & & & & & & & & & 72.5 & \\
\hline $\mathbf{y}$ & $y, f l r$ & 3.2 & & 6.6 & 8.5 & & & & & & & & & & & & & & & \\
\hline z & $\mathrm{x}, \mathrm{m}$ & 2.95 & 5.6 & & 8.5 & 11.2 & 14.6 & 17 & 19.9 & & 25.3 & & & & & & & & & \\
\hline$z$ & $y, m$ & 2.95 & 5.6 & & 8.5 & 11.2 & 14.6 & 17 & 19.9 & 22.5 & 25.3 & & & & & 37.9 & & & 75.9 & \\
\hline $\mathrm{z}$ & $\mathrm{z}, \mathrm{m}$ & 2.95 & 5.6 & & 8.5 & 11.2 & 14.6 & 17.5 & 19.9 & 22.5 & 25.6 & & & & & & & & & \\
\hline 2 & $x, g$ & 2.95 & 5.6 & & 8.5 & 11.2 & 14.6 & 17.2 & 19.9 & 22.5 & 25.3 & & & & & & & & & \\
\hline z & $y, g$ & 2.95 & 5.6 & & 8.5 & 11.2 & 14.6 & 17.2 & 19.9 & 22.5 & 25.3 & & & & & & & & & \\
\hline $\mathbf{z}$ & $\mathrm{z}, \mathrm{g}$ & 2.95 & 5.6 & & 8.5 & 11.2 & 14.6 & 17.2 & 19.9 & 22.5 & 25.3 & & & & & & & & & \\
\hline $\mathbf{z}$ & $y, f l r$ & 2.95 & & 6.3 & 8.05 & & & & & & & & & & & & & & & \\
\hline Amb.c & $\mathrm{x}, \mathrm{m}$ & 3.2 & & 6.1 & 8.05 & 11.2 & 14.6 & & & & & & & & & & & & & \\
\hline $\mathrm{Amb}$ & $y, m$ & & & 6.1 & 8.05 & 11.2 & & & & & & & & & & & & & & \\
\hline $\mathrm{Amb}$ & $\mathrm{z}, \mathrm{m}$ & & & 6.1 & 8.05 & 11.2 & & & & & & & & & & & & & & \\
\hline $\mathrm{Amb}$ & $x, g$ & & & 6.1 & 8.05 & 11.2 & & & & & & & & & & & & & & \\
\hline $\mathrm{Amb}$ & $\mathrm{y}, \mathrm{g}$ & & & 6.1 & 8.05 & 11.2 & & & & & & & & & & & & & & \\
\hline Amb. & $\mathrm{z}, \mathrm{g}$ & & & 6.1 & 8.05 & 11.2 & & & & & & & & & & & & & & \\
\hline Amb. & $y, f \mid r$ & 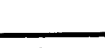 & 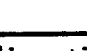 & 6.4 & 8.05 & & & & & & & & & & & & & & & \\
\hline
\end{tabular}


Table 2. Frequencies $(\mathrm{Hz})$ at which peaks were measured for horizontal corrector-magnet/girder assembly under various conditions

\begin{tabular}{|c|c|c|c|c|c|c|c|c|c|c|c|c|c|c|c|c|c|c|c|c|}
\hline $\begin{array}{l}\text { Exc. } \\
\text { Dir. } 2\end{array}$ & $\begin{array}{l}\text { Meas. } \\
\text { Dir. and } \\
\text { Locatio }\end{array}$ & & & & & & & & Fre & $\begin{array}{r}\text { quen } \\
(\mathrm{Hz})\end{array}$ & & & & & & & & & & \\
\hline$x$ & $x, m$ & 2.95 & & 5.87 & & 8.5 & 11.2 & 13.6 & & 17.5 & & 36.1 & 40 & & 48.5 & 57.9 & 62.3 & & & \\
\hline $\mathbf{x}$ & $y, m$ & 2.72 & & 5.87 & & 8.3 & 11 & 14.1 & 16.5 & & & 36.1 & 40 & & 48.5 & 57.9 & 62.3 & 72.5 & & \\
\hline$x$ & $\mathrm{z}, \mathrm{m}$ & 2.72 & & 5.87 & & 8.3 & 11 & 14.6 & & & & & & & & 57.9 & & & & 88.7 \\
\hline$x$ & $x, g$ & 2.72 & & 5.87 & & 8.3 & 11 & 13.8 & & 17.5 & & 35.9 & 39.8 & & & 57.9 & 62.3 & & & 87.2 \\
\hline$x$ & $y, g$ & 2.72 & & 5.87 & & 8.3 & 11 & 14.3 & & & & & & & & & & & & 87.2 \\
\hline$x$ & $2, \mathrm{~g}$ & 2.72 & & 5.87 & & 8.3 & 11 & 14.3 & & & & & & & & & & 73.5 & & 84.6 \\
\hline$x$ & $y, f l r$ & 2.72 & & & 6.1 & 8.05 & & & & & & & & & & & & & & 87.2 \\
\hline$y$ & $x, m$ & 2.7 & & 5.87 & & 8.5 & 11 & 14.6 & & & 33.4 & 36.4 & 40 & 43 & & & & & & 89.2 \\
\hline$y$ & $\mathrm{y}, \mathrm{m}$ & 2.7 & & 5.87 & & 8.5 & 11 & 14.6 & & & 33.4 & 36.4 & 40 & 43 & & & 62.4 & & & 89.2 \\
\hline$y$ & $\mathrm{z}, \mathrm{m}$ & 2.7 & & 5.87 & & 8.5 & 11 & 14.6 & & 18 & & & & & & & & & & 89.2 \\
\hline$y$ & $\mathrm{x}, \mathrm{g}$ & 2.7 & & 5.87 & & 8.5 & 11.2 & 14.1 & & & 33.5 & 36.1 & & & & & & & & \\
\hline$y$ & $y, g$ & 2.7 & & 5.87 & & 8.5 & 11 & 14.1 & & 18.2 & & & & & & & & & & 88.8 \\
\hline$y$ & $\mathrm{z}, \mathrm{g}$ & 2.7 & & 5.87 & & 8.5 & 11 & 14.1 & & 18.2 & & & & & & & & & & \\
\hline$y$ & $y, f l r$ & 3.2 & & & 6.35 & 8.05 & 11 & & & & & & & & & & & & & \\
\hline z & $\mathbf{x}, \mathrm{m}$ & 2.72 & & 5.87 & & 8.5 & 11.2 & 14.3 & 16.7 & & & 36.1 & & 43.4 & & 58.2 & & & 79.5 & 90.2 \\
\hline 2 & $y, m$ & 2.72 & & 5.87 & & 8.5 & 11.2 & 14.3 & & 17.7 & & & & 43.1 & & & 64.2 & & 79.5 & 82.2 \\
\hline$z$ & $\mathrm{z}, \mathrm{m}$ & 2.72 & 4.4 & 5.87 & & 8.5 & 11.2 & 14.3 & & 17.7 & & & & & & & & & & 82.2 \\
\hline z & $x, g$ & 2.72 & & 5.87 & & 8.5 & 11.2 & 14.3 & 16.7 & & & & & & & 58 & & & 79.5 & \\
\hline $\mathbf{z}$ & $y, g$ & 2.72 & & 5.87 & & 8.5 & 11.2 & 14.3 & 16.7 & & & & & & & & & & & 88.8 \\
\hline z & $\mathrm{z}, \mathrm{g}$ & 2.95 & 4.4 & 5.87 & & 8.5 & 11.2 & 14.3 & & 17.7 & & & & & & & & 74.2 & 79.3 & 82.4 \\
\hline z & $y, f l r$ & 3.2 & & & 6.4 & 8.05 & & & & & & & & & & & & & & \\
\hline Amb.c & $\mathrm{x}, \mathrm{m}$ & 3.2 & & & 6.1 & 8.05 & & & & & & & & & & & & & & \\
\hline Amb. & $y, m$ & 3.2 & & & 6.1 & 8.05 & & & & & & & & & & & & & & \\
\hline $\mathrm{Amb}$ & $\mathrm{z}, \mathrm{m}$ & 3.2 & & & 6.1 & 8.05 & & & & & & & & & & & & & & \\
\hline $\mathrm{Amb}$ & $x, g$ & 2.95 & & & 6.4 & & 11.2 & & & & & & & & & & & & & \\
\hline Amb. & $y, g$ & 2.95 & & & 6.4 & 8.3 & 11.2 & & & & & & & & & & & & & \\
\hline Amb. & $\mathrm{z}, \mathrm{g}$ & & & & & 8.3 & 11.2 & & & & & & & & & & & & & \\
\hline A mb. & $y, f 1 r$ & 3.2 & & & 6.35 & 8.05 & & & & & & & & & & & & & & \\
\hline
\end{tabular}

axc. Dir. = excitation direction.

$\mathrm{b}_{\mathrm{m}}=$ magnet, $\mathrm{g}=$ girder, flr. = floor.

cAmb. = ambient. 
Table 3. Frequencies $(\mathrm{Hz})$ at which peaks were measured for quadrupole-magnet/girder assembly under various conditions

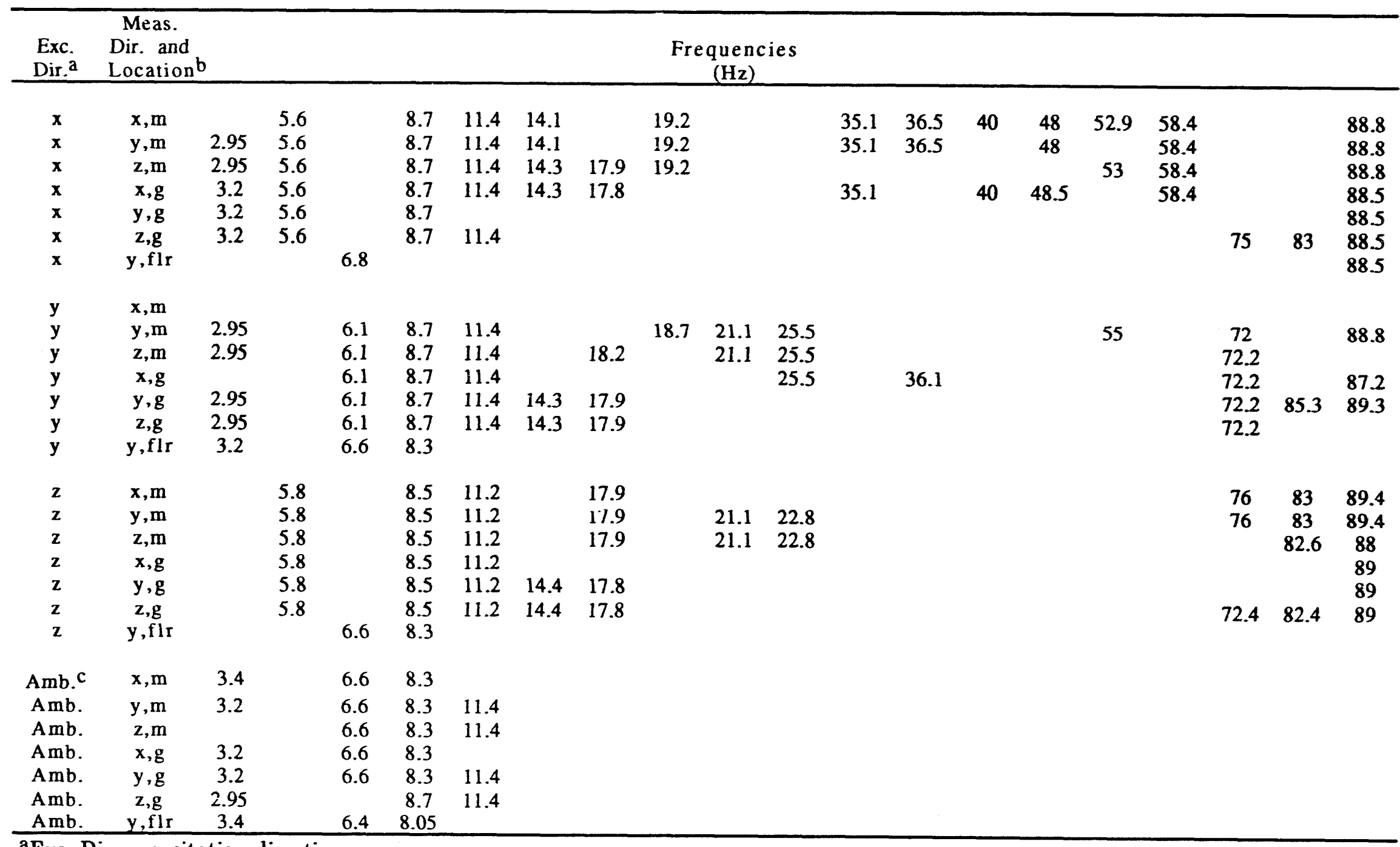

aExc. Dir. = excitation direction.

$b_{m}=$ magnet, $g=$ girder, flr. $=$ floor.

cAmb. = ambient. 
Table 4. Frequencies $(\mathrm{Hz})$ at which peaks were measured for sextupole-magnet/girder assembly under various conditions

\begin{tabular}{|c|c|c|c|c|c|c|c|c|c|c|c|c|c|c|c|c|c|c|c|c|}
\hline $\begin{array}{l}\text { Exc. } \\
\text { Dir. }^{\mathrm{a}}\end{array}$ & $\begin{array}{l}\text { Meas. } \\
\text { Dir. and } \\
\text { Location }\end{array}$ & & & & & & & & Fre & $\begin{array}{c}\text { quenc } \\
(\mathrm{Hz})\end{array}$ & ies & & & & & & & & & \\
\hline$x$ & $\begin{array}{l}y, \mathrm{~m} \\
\mathrm{z}, \mathrm{m}\end{array}$ & & & 6.1 & & & $\begin{array}{l}8.5 \\
8.5\end{array}$ & $\begin{array}{l}11.6 \\
11.6\end{array}$ & & & $\begin{array}{l}35.4 \\
35.4\end{array}$ & $\begin{array}{l}39 \\
39\end{array}$ & 43.4 & & 52 & $\begin{array}{l}58 \\
58\end{array}$ & 62.3 & 86 & $\begin{array}{l}89 \\
89\end{array}$ & \\
\hline $\mathbf{x}$ & $x, g$ & & & & & & 8.5 & 11.6 & 18.2 & & 35.4 & 39 & & & & 58 & & 86 & & 95 \\
\hline$x$ & $y, g$ & & & 61 & & & 8.5 & 11.6 & & & & & & & & & & & 89 & \\
\hline $\begin{array}{l}\mathbf{x} \\
\mathbf{x}\end{array}$ & $\begin{array}{c}z, g \\
y, f l r\end{array}$ & 3.21 & 4.63 & 0.1 & 6.6 & & 8.5 & & & & & & & & & & 74 & 85 & & 94 \\
\hline $\mathbf{y}$ & $\mathrm{x}, \mathrm{m}$ & & 4.4 & & & 7.5 & 8.5 & 11.4 & & 29.6 & 36.1 & 40 & & 49 & 54.5 & & & 84 & & 95.2 \\
\hline $\mathbf{y}$ & $y, m$ & & 4.4 & & & 7.5 & 8.5 & 11.4 & & & 36.1 & 40 & & & & & & & 89 & 95.2 \\
\hline $\mathbf{y}$ & $\mathrm{z}, \mathrm{m}$ & & 4.4 & & & 7.5 & 8.5 & 11.4 & & & 36.1 & 40 & 43 & & 54.5 & & & 84 & & 95.2 \\
\hline y & $x, g$ & & & & & & & & 17.5 & 29.5 & 36 & & & & & & & 85 & & 95.2 \\
\hline $\begin{array}{l}y \\
y\end{array}$ & $\begin{array}{l}y, g \\
z, g\end{array}$ & & & & & 75 & & 117 & 17.5 & & & & & & & & 81 & & 88 & 95.2 \\
\hline $\begin{array}{l}y \\
y\end{array}$ & $\begin{array}{c}z, g \\
y, f ! r\end{array}$ & & 4.65 & & & 7.5 & 8.5 & 11.1 & & & & & & & & & & 83 & & 95 \\
\hline $\mathbf{z}$ & $\mathbf{x}, \mathbf{m}$ & & & & & & & 114 & & & & & & & & & & & & \\
\hline z & $y, m$ & 2.95 & 4.4 & & & $\begin{array}{l}7.5 \\
7.5\end{array}$ & $\begin{array}{l}8.3 \\
8.3\end{array}$ & $\begin{array}{l}11.4 \\
11.4\end{array}$ & $\begin{array}{l}17.8 \\
17.8\end{array}$ & 30.3 & 35.9 & & 42.7 & 49 & 53 & & & 83.4 & 87.3 & 95.8 \\
\hline $\mathbf{z}$ & $\mathrm{z}, \mathrm{m}$ & 2.95 & 4.4 & & & $\begin{array}{l}7.5 \\
7.5\end{array}$ & $\begin{array}{l}8.3 \\
8.3\end{array}$ & 11.4 & 17.8 & & 36.6 & & & & & & 80.9 & & 89.2 & \\
\hline $\mathbf{z}$ & $x, g$ & 2.95 & & & & 7.5 & 8.3 & 11.4 & & 30.3 & & & 42.7 & & 52.8 & & & 85.3 & & \\
\hline $\mathbf{z}$ & $y, g$ & & & & & 7.5 & 8.3 & 11.4 & & & & & & & & & & 82.2 & 87 & 95.5 \\
\hline $\mathbf{z}$ & $\mathrm{z}, \mathrm{g}$ & 2.95 & & & & 7.5 & 8.3 & 11.4 & 17.8 & & & & & & & & 80.2 & & 88 & \\
\hline $\mathbf{z}$ & $y, f l r$ & 3.2 & & 6.3 & & & & & & & & & & & & & & & 84 & 96 \\
\hline$A m b$ & & & & & & & & & & & & & & & & & & & & \\
\hline & $\mathrm{x}, \mathrm{m}$ & 2.9 & & 6.2 & & & 8 & $\begin{array}{l}12.1 \\
11.2\end{array}$ & & & & & & & & & & & & \\
\hline $\begin{array}{l}\text { Amb. } \\
\text { Amb. }\end{array}$ & $y, m$ & & & 6.2 & & & 8.3 & $\begin{array}{l}11.2 \\
11.7\end{array}$ & & & & & & & & & & & & \\
\hline $\begin{array}{l}\text { Amb. } \\
\text { Amb. }\end{array}$ & $\begin{array}{l}\mathrm{z}, \mathrm{m} \\
\mathrm{x}, \mathrm{g}\end{array}$ & 2.9 & & 6.1 & & & 8.3 & 11.7 & & & & & & & & & & & & \\
\hline Amb. & $\begin{array}{l}x, g \\
y, g\end{array}$ & $\begin{array}{l}2.9 \\
2.9\end{array}$ & & 6.1 & & & & 11.2 & & & & & & & & & & & & \\
\hline Amb. & $\mathrm{z}, \mathrm{g}$ & & & & & 7.5 & 8.3 & & & & & & & & & & & & & \\
\hline Amb. & $y, f l r$ & 2.9 & & 6.2 & & & 8 & 12.1 & & & & & & & & & & & & \\
\hline
\end{tabular}

Amb. $\quad$ y, fir $\quad 2.9 \quad 6.2$

$\mathrm{b}_{\mathrm{m}}=$ magnet, $\mathrm{g}=$ girder, fl $\mathrm{r}=$ floor.

$c_{\text {Amb. }}=$ ambient. 
Table 5. Frequencies $(\mathrm{Hz})$ at which peaks were measured for various magnet assemblies and excitation directions under impact excitation

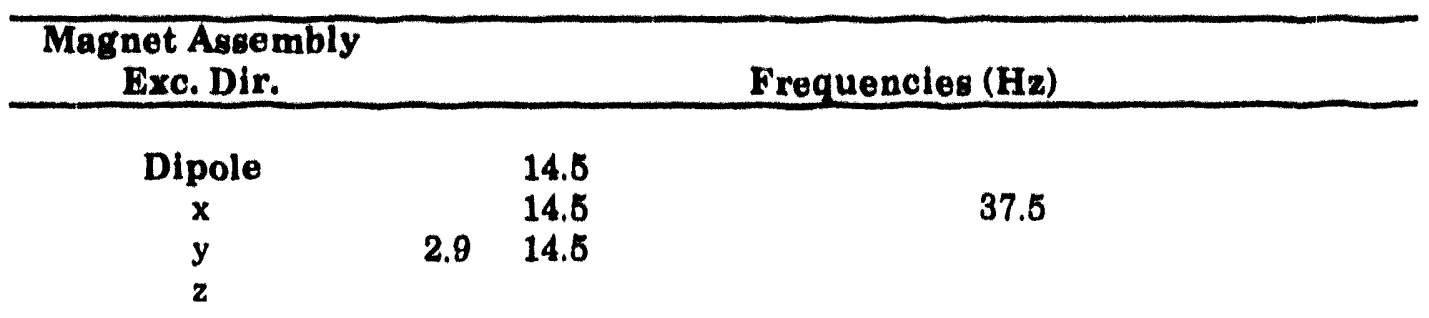

Horizontal corrector

$\begin{array}{ll}\mathrm{x} & 12 \\ \mathrm{y} & 12 \\ \mathrm{z} & 12\end{array}$

Quadrupole

$\begin{array}{llll}\mathrm{x} & 19 & & 63 \\ \mathrm{y} & 19 & & 54 \\ \mathrm{z} & 19 & 20.5 & 53\end{array}$

Sextupole

$x$

35.9

y

35.9 
Table 6. Measured RMS displacements $(\mu)$, within the frequency range $1.100 \mathrm{~Hz}$

\begin{tabular}{|c|c|c|c|c|c|c|c|}
\hline $\begin{array}{l}\text { Assembly } \\
\text { Exc. Dir. }\end{array}$ & $\begin{array}{c}X, \\
\text { magnet }\end{array}$ & $\begin{array}{c}Y, \\
\text { marnet }\end{array}$ & $\begin{array}{c}\mathbf{Z} \\
\text { mapuet } \\
\end{array}$ & $\begin{array}{c}\mathbf{X}, \\
\text { firder }\end{array}$ & $\begin{array}{c}\text { Y, } \\
\text { pirder }\end{array}$ & $\begin{array}{c}z_{1} \\
\text { irder }\end{array}$ & $\begin{array}{c}\mathbf{Y} \\
\text { noor }\end{array}$ \\
\hline \multicolumn{8}{|l|}{ Dipole } \\
\hline $\mathbf{x}$ & 0.164 & 0.17 & 0.141 & 0.197 & 0.167 & 0.14 & 0.17 \\
\hline $\mathbf{Y}$ & 0.153 & 0.146 & 0.124 & 0.197 & 0.148 & 0.0974 & 0.183 \\
\hline $\mathbf{z}$ & 0.206 & 0.198 & 0.178 & 0.263 & 0.188 & 0.164 & 0.171 \\
\hline Ambient & 0.122 & 0.116 & 0.0908 & 0.161 & 0.11 & 0.0610 & 0.161 \\
\hline \multicolumn{8}{|c|}{ Horizontal corrector } \\
\hline $\mathbf{x}$ & 0.167 & 0.171 & 0.142 & 0.223 & 0.291 & 0.126 & 0.207 \\
\hline $\mathbf{Y}$ & 0.208 & 0.202 & 0.188 & 0.237 & 0.248 & 0.182 & 0.166 \\
\hline $\mathbf{z}$ & 0.188 & 0.201 & 0.187 & 0.835 & 1.68 & 0.182 & 0.268 \\
\hline Ambient & 0.106 & 0.101 & 0.0814 & 0.238 & 0.462 & 0.0531 & 0.274 \\
\hline \multicolumn{8}{|c|}{ Quadrupole } \\
\hline $\mathbf{x}$ & 0.185 & 0.191 & 0.174 & 0.42 & 0.80 & 0.161 & 0.179 \\
\hline $\mathbf{Y}$ & $\mathbf{x x x}$ & 0.165 & 0.139 & 0.203 & 0.160 & 0.126 & 0.166 \\
\hline $\mathbf{z}$ & 0.207 & 0.117 & 0.112 & 0.663 & 1.27 & 0.0866 & 0.274 \\
\hline Ambient & 0.105 & 0.1 & 0.0782 & 0.178 & 0.12 & 0.0611 & 0.147 \\
\hline \multicolumn{8}{|l|}{ Sextupole } \\
\hline $\mathbf{x}$ & 0.142 & 0.133 & 0.138 & 0.466 & 1.56 & 0.217 & 0.291 \\
\hline $\mathbf{Y}$ & 0.789 & 0.32 & 0.638 & 2.74 & 3.26 & 0.426 & 1.106 \\
\hline $\mathbf{Z}$ & 0.44 & 0.244 & 0.337 & 0.458 & 1.02 & 0.196 & 0.223 \\
\hline Ambient & 0.138 & 0.0907 & 0.0977 & 0.252 & 0.127 & 0.208 & 0.108 \\
\hline
\end{tabular}


Table 7. Frequencies at which responses were measured and response source for a synchrotron magnet/ girder assembly

\begin{tabular}{cc}
\hline $\begin{array}{c}\text { Reopones } \\
\text { Rrequenoy (H) }\end{array}$ & Regponse Source \\
\hline $2.7-3.2$ & Ambient \\
$\mathbf{5 . 6 - 6 . 1}$ & $?$ \\
$6.1-6.6$ & Ambient \\
7.5 & Ambient \\
$8-8.7$ & Ambient \\
$11-11.4$ & Ambient \\
12 & Horizontal corrector magnet \\
$14.1-14.13$ & Dipole magnet \\
$17-18.2$ & Horizontal corrector magnet \\
$19.2-19.9$ & Quadrupole magnet \\
$21-21.2$ & Quadrupole magnet \\
22.5 & Dipole magnet \\
25.3 & Dipole magnet \\
$35.4-36.6$ & Sextupole magnet \\
$39-40$ & Horizontal corrector magnet \\
$42-43$ & Sextupole magnet \\
48 & Sextupole/horizontal corrector magnets \\
\hline
\end{tabular}




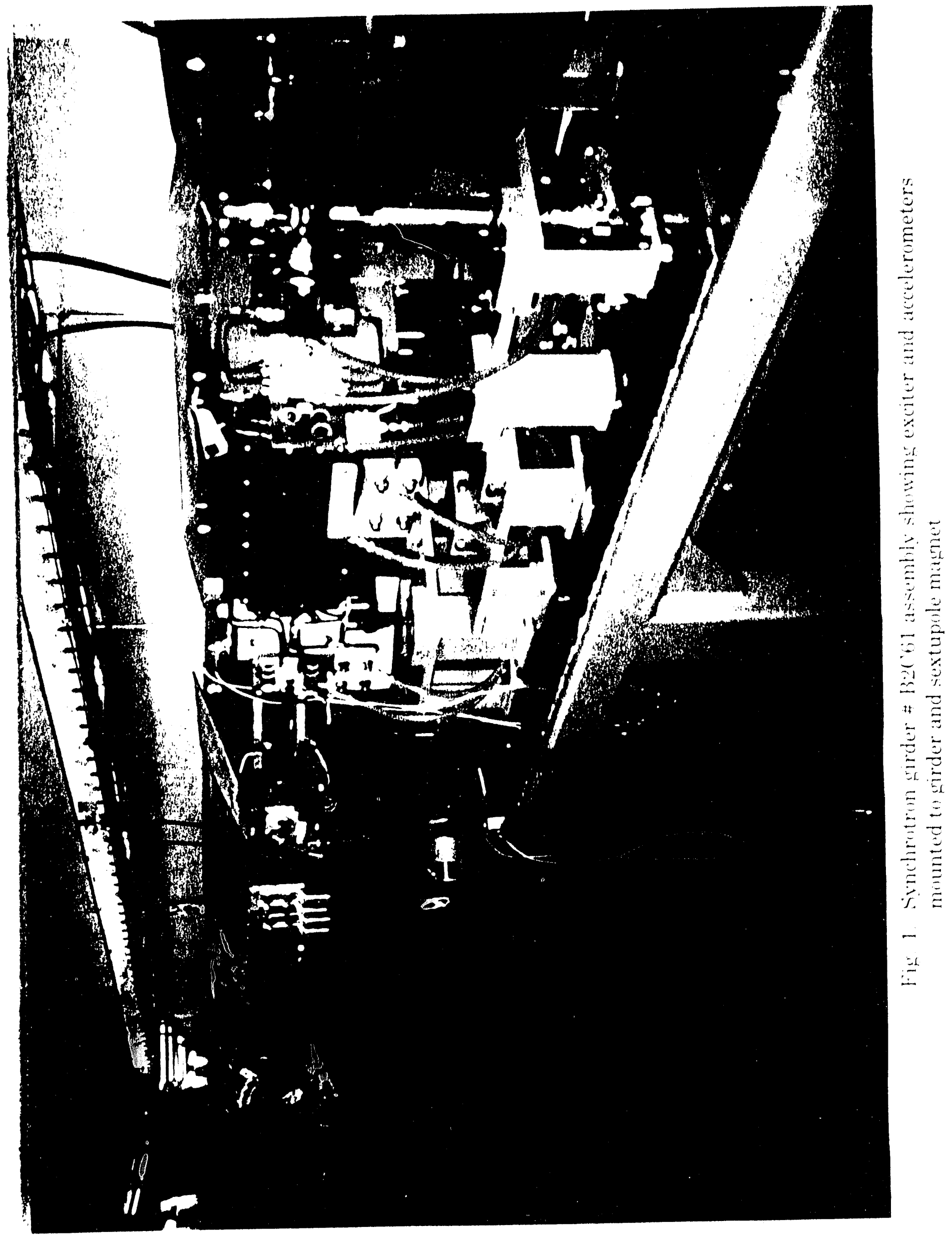


Triaxial accelerometer mount
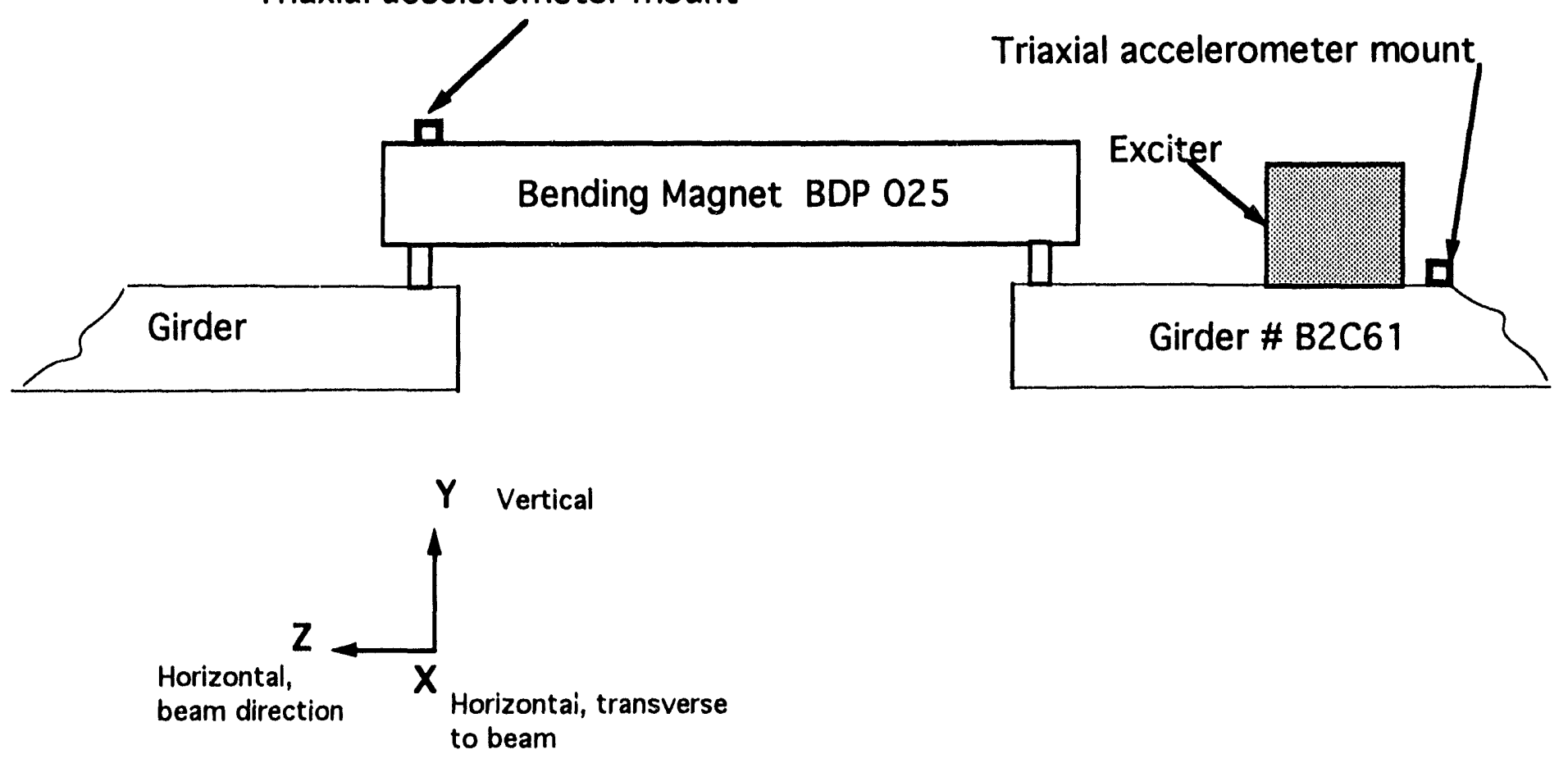

Measurement directions

Fig. 2. Accelerometer location on bending-magnet/girder assembly 

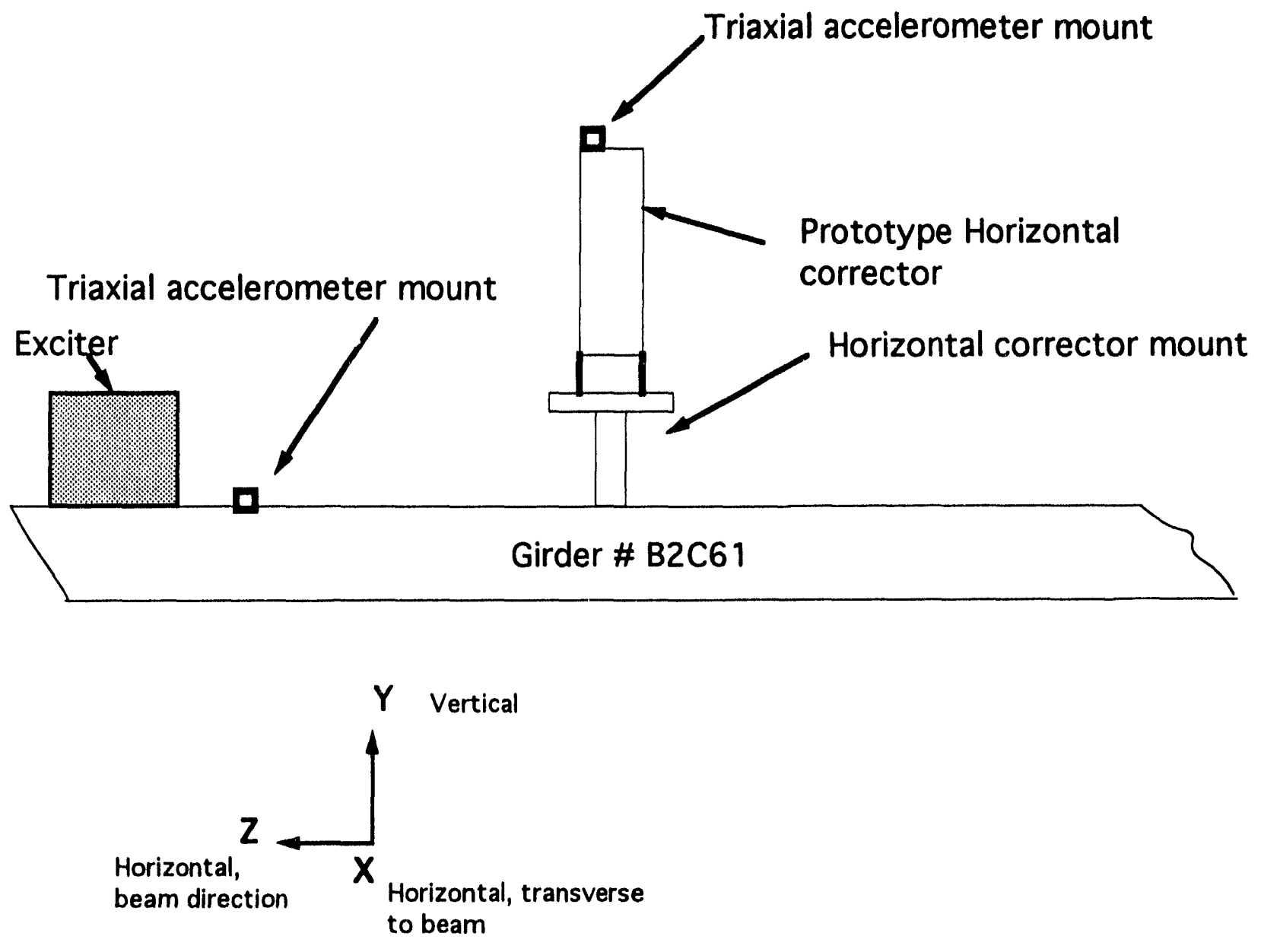

Measurement directions

Fig. 3. Accelerometer location on horizontal corrector-magnet/girder assembly 

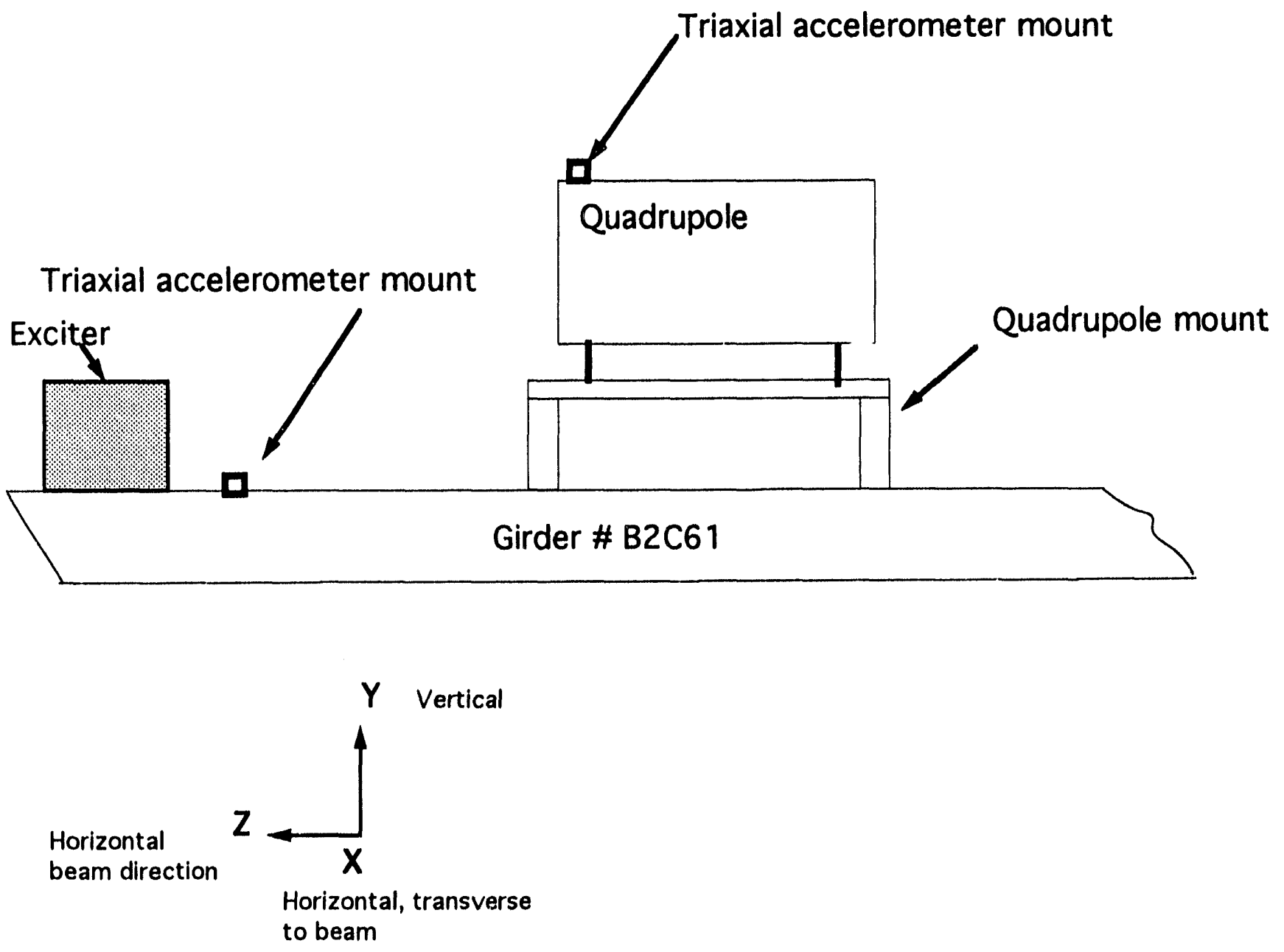

Measurement directions

Fig. 4. Accelerometer location on quadrupole-magnet/girder assembly 

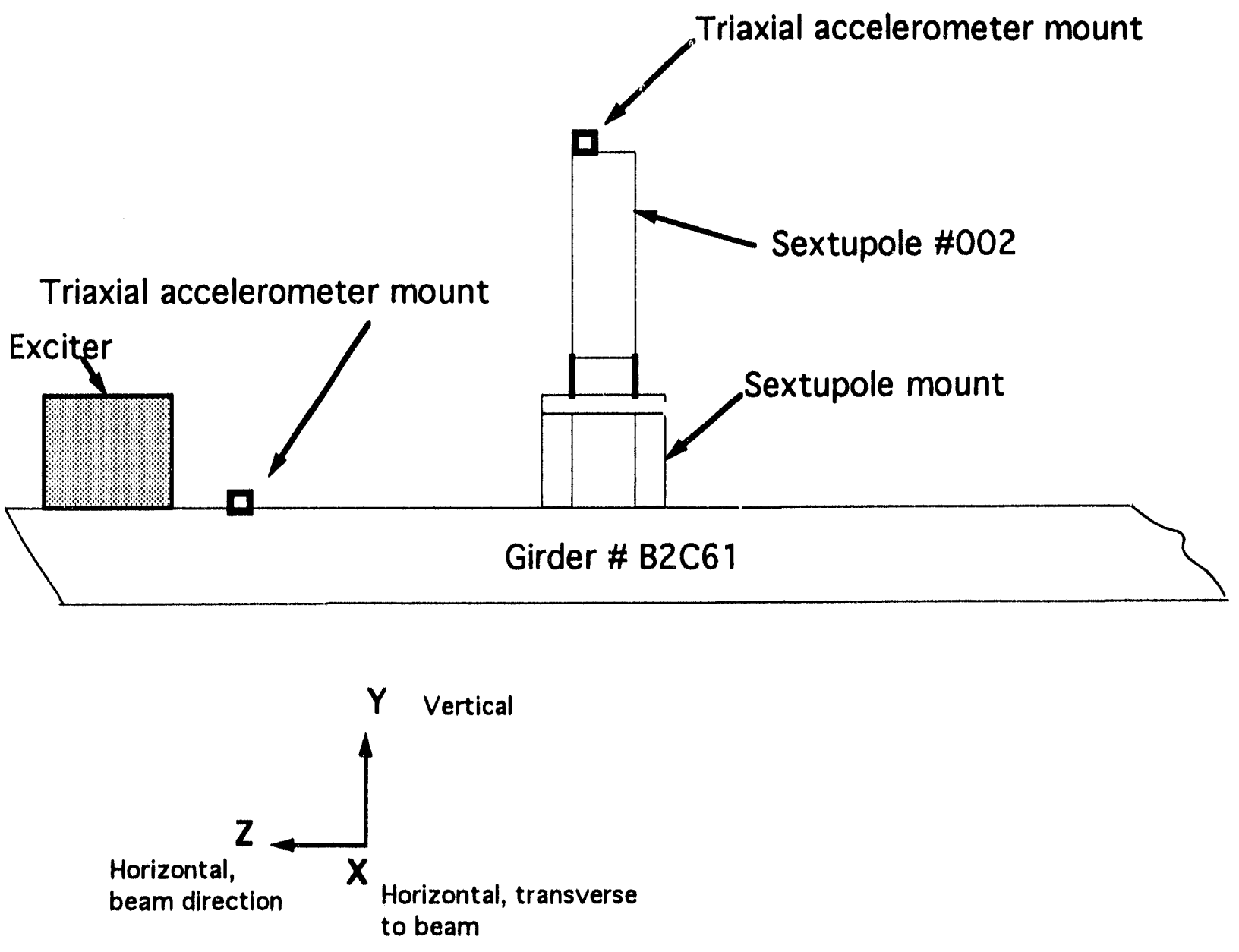

Measurement directions

Fig. 5. Accelerometer location on sextupole-magnet/girder assembly 
Appendix A: Bending (dipole) magnet response 
A-1

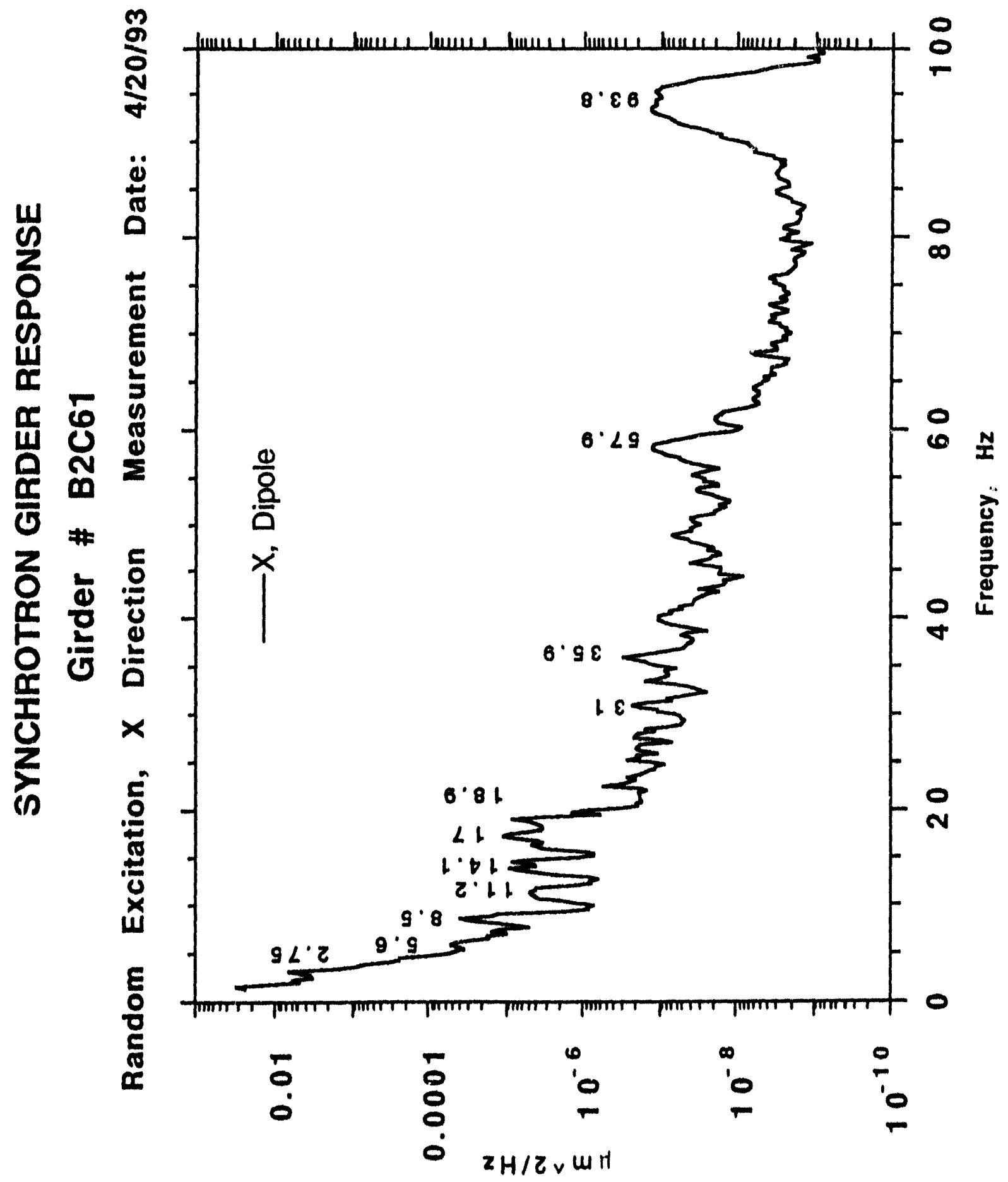




\section{A-2}

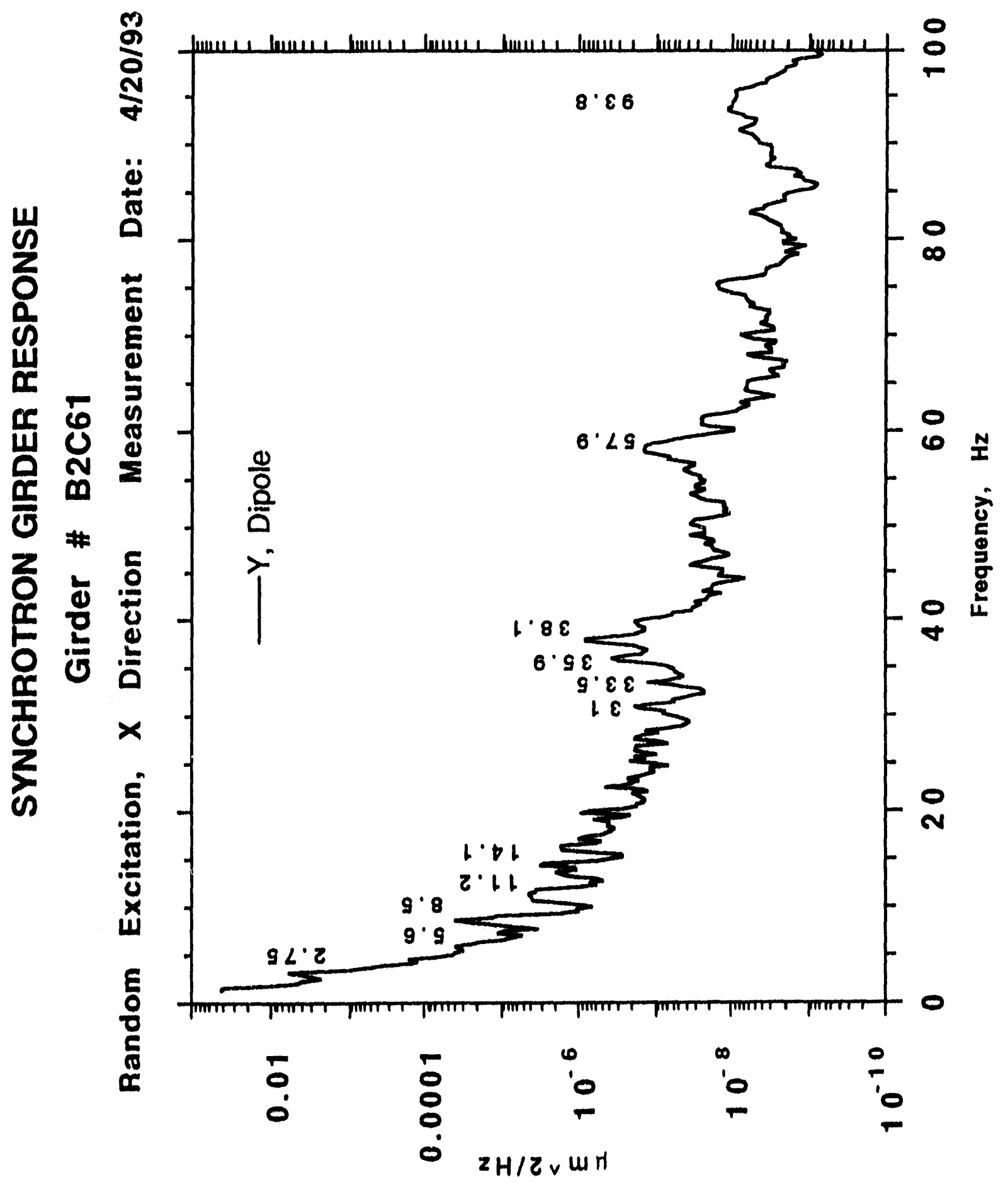




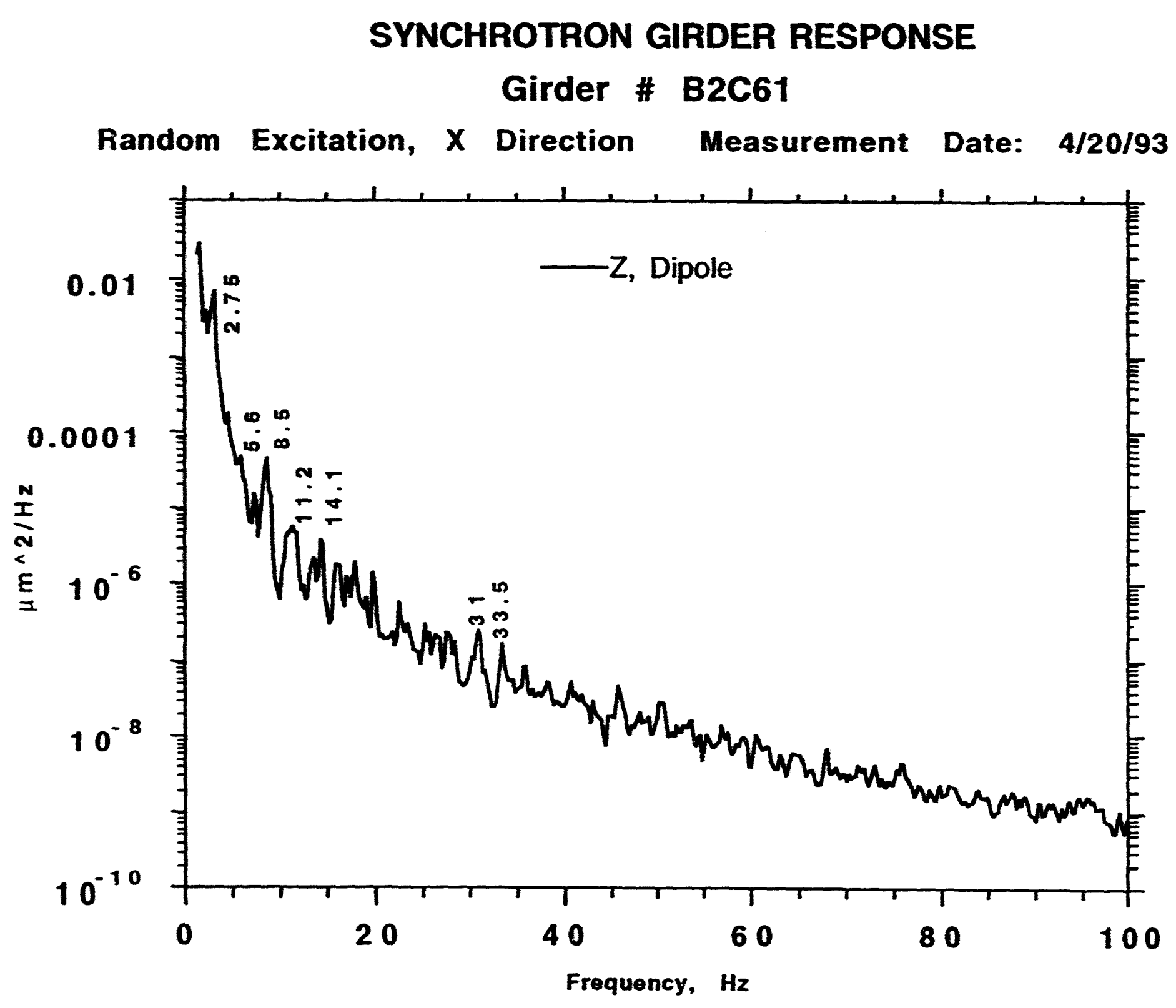




\section{A-4}

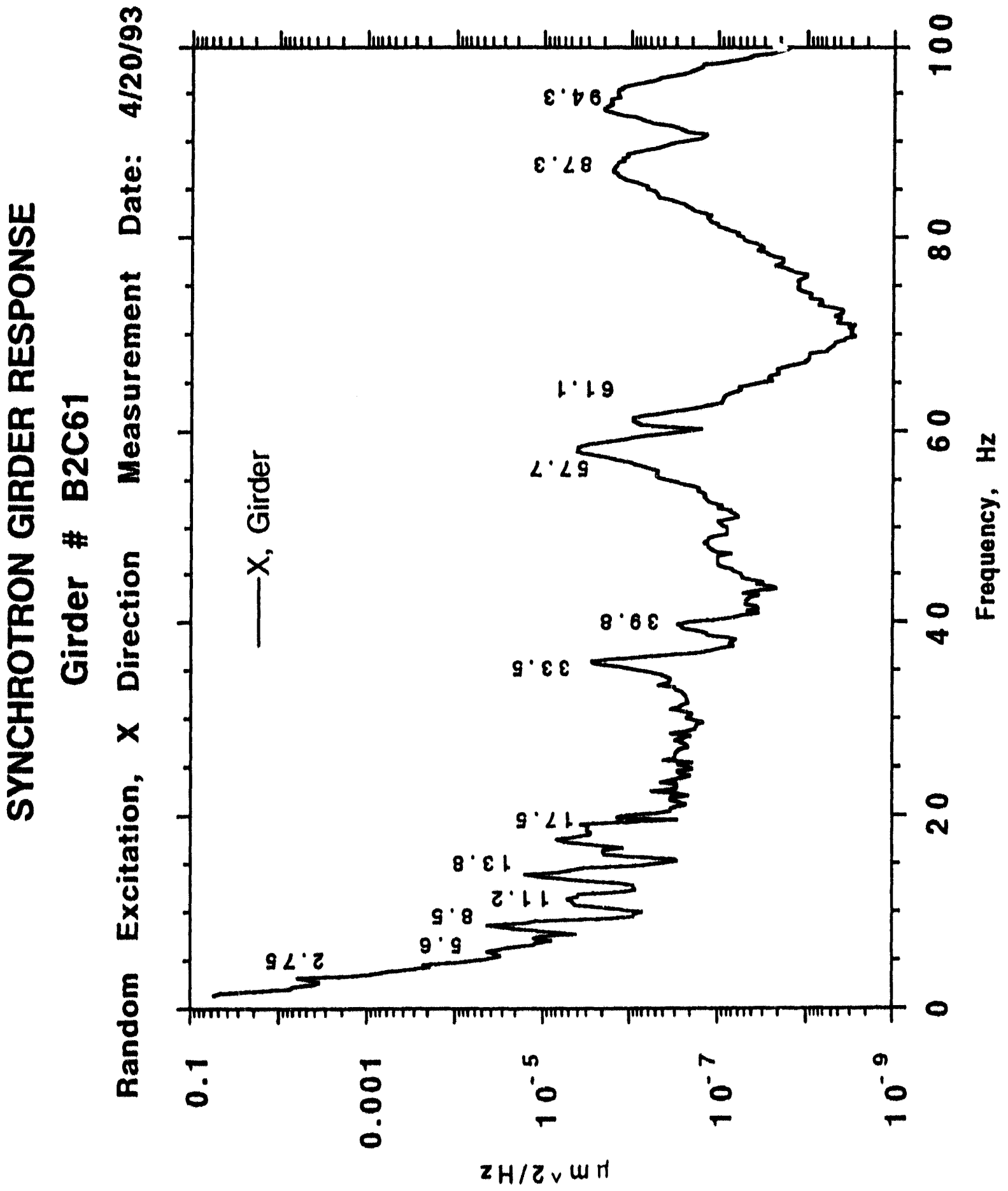




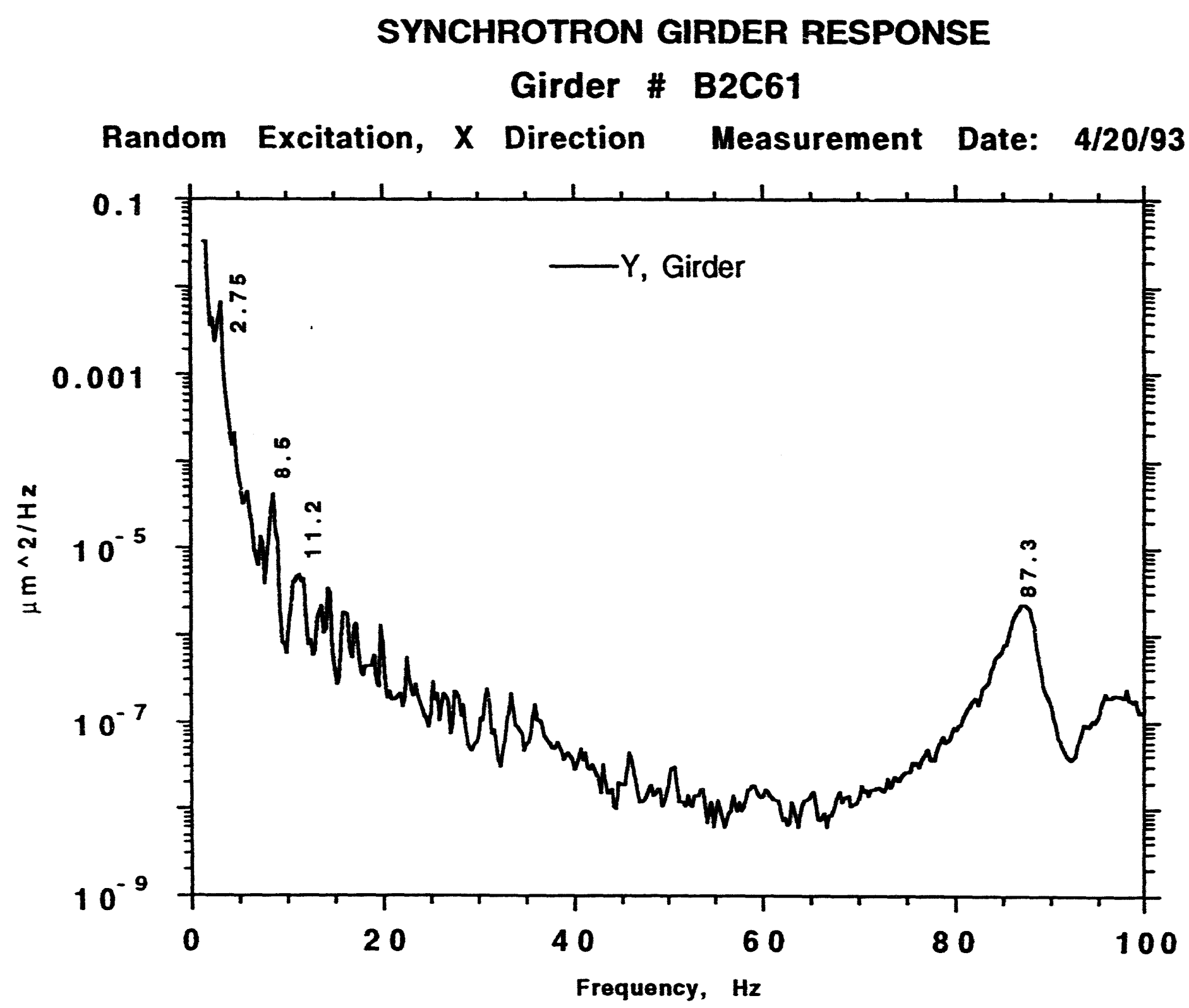




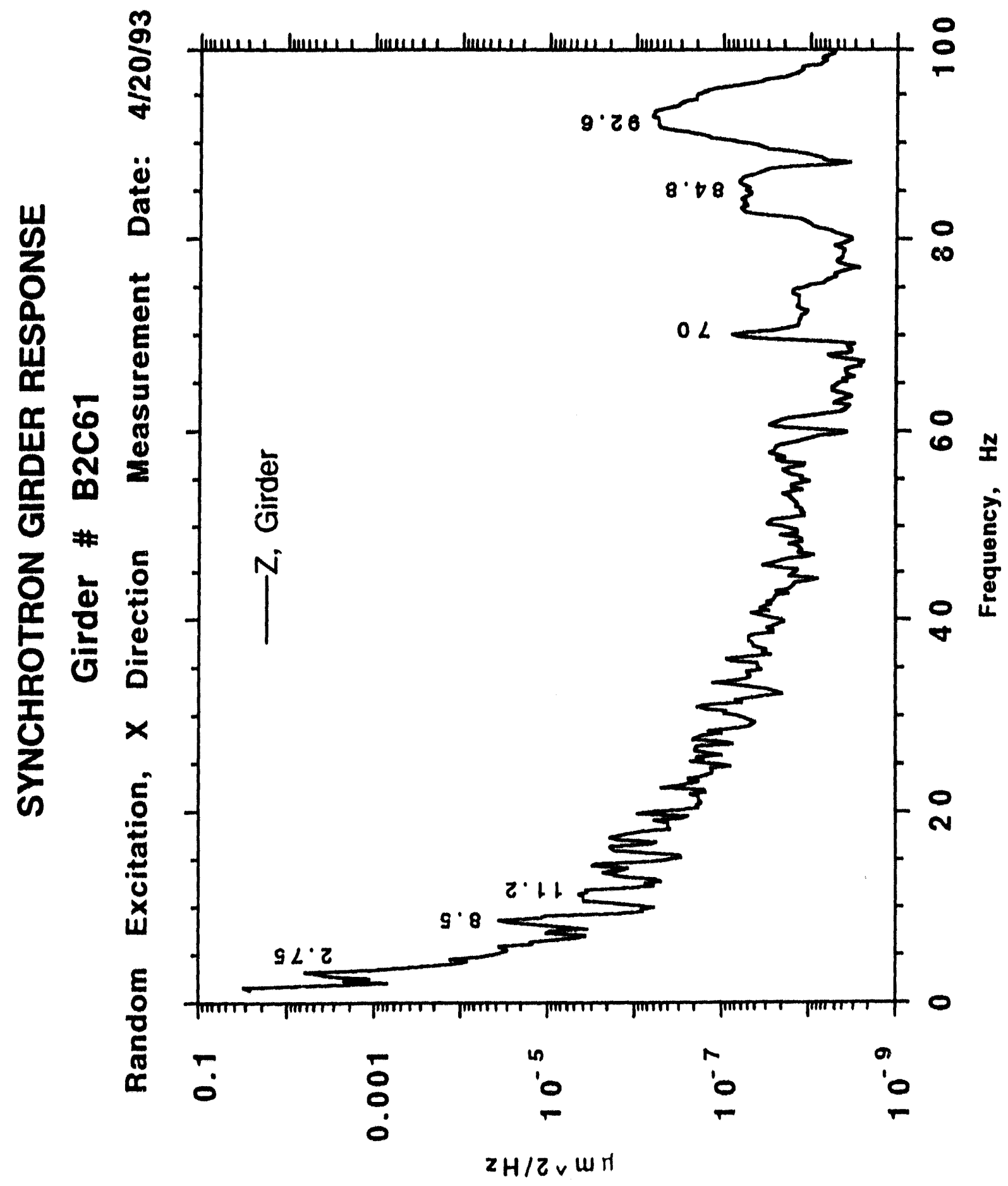




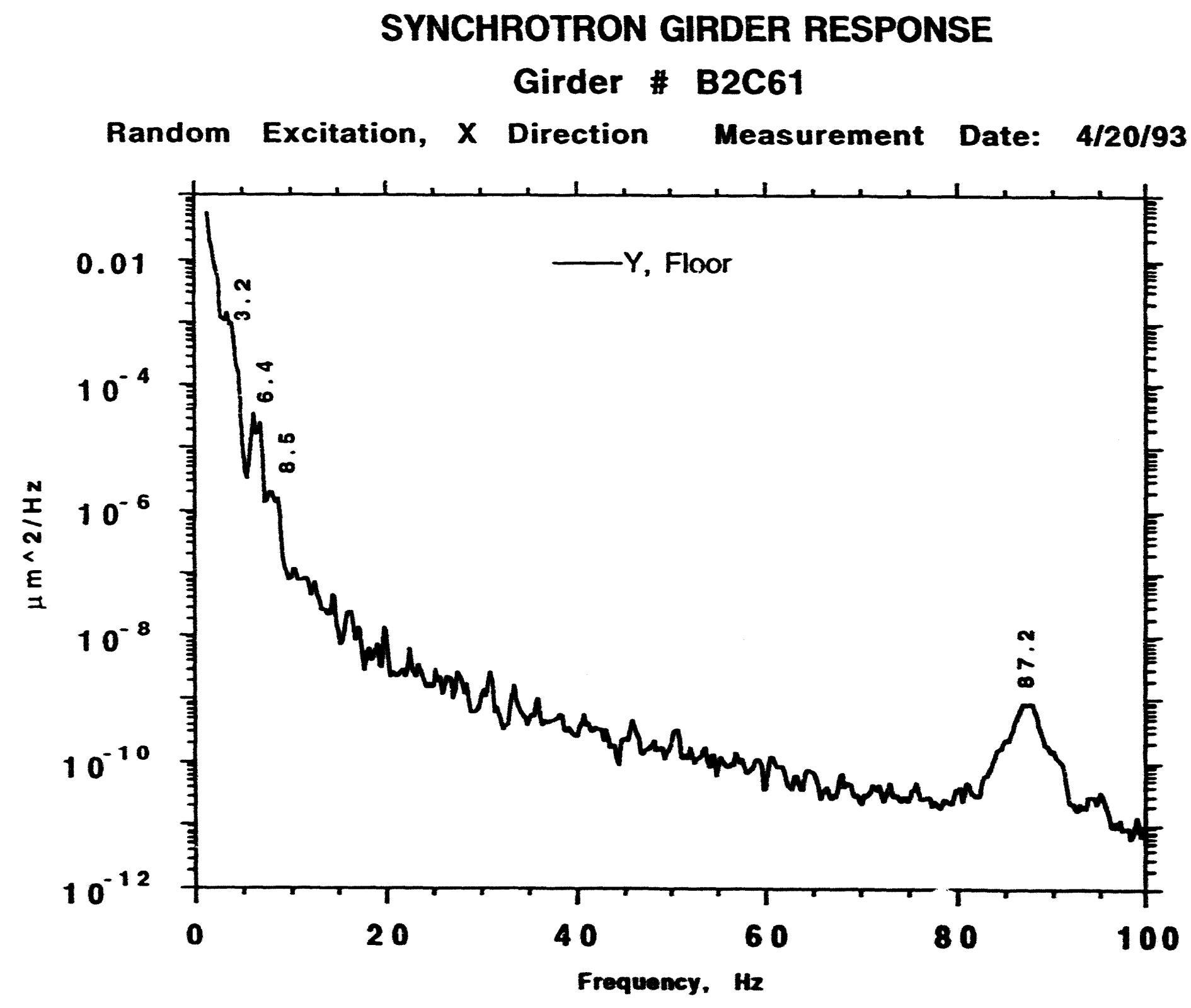




\section{SYNCHROTRON GIRDER RESPONSE}

Girder \# B2C61

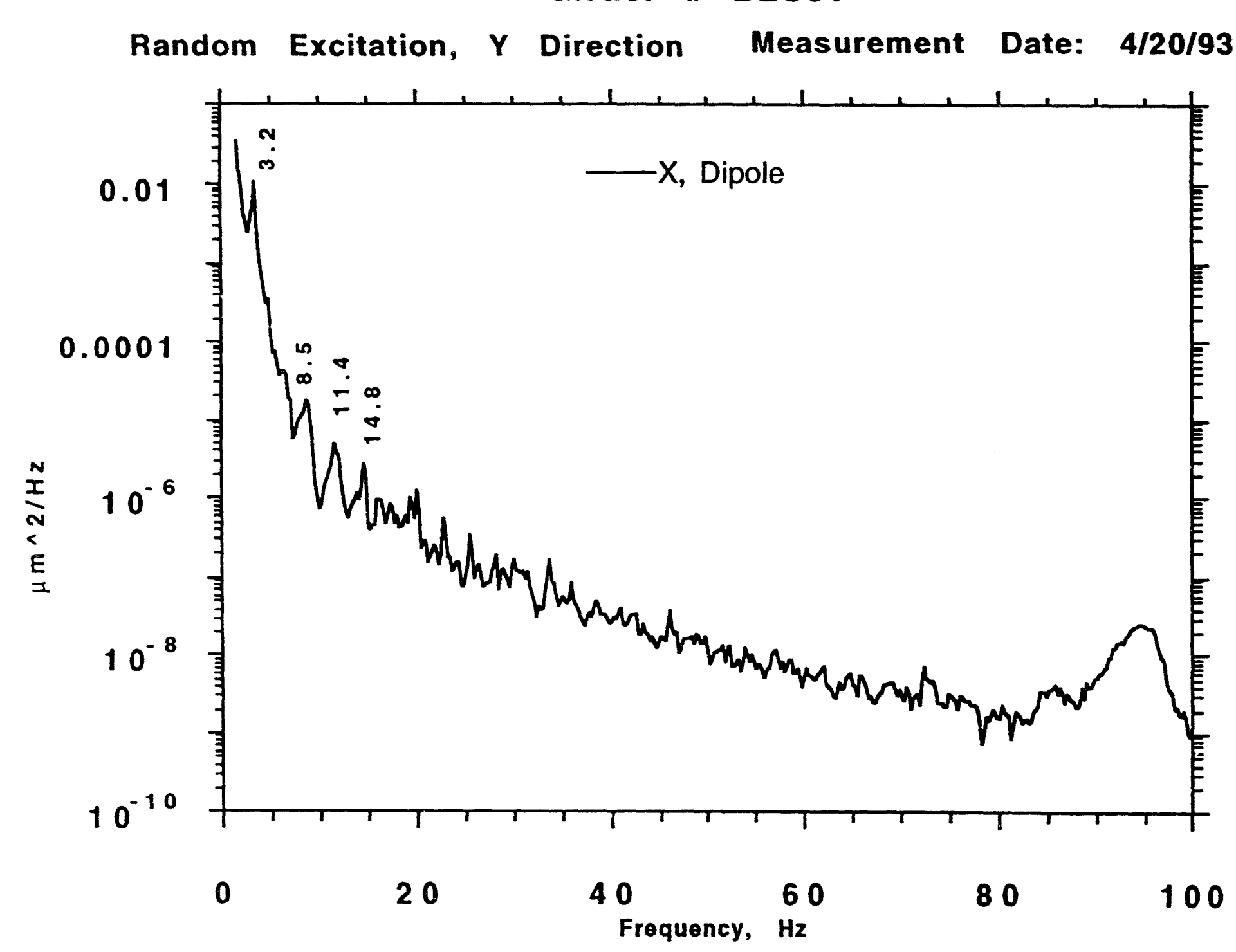




\section{SYNCHROTRON GIRDER RESPONSE}

Girder \# B2C61

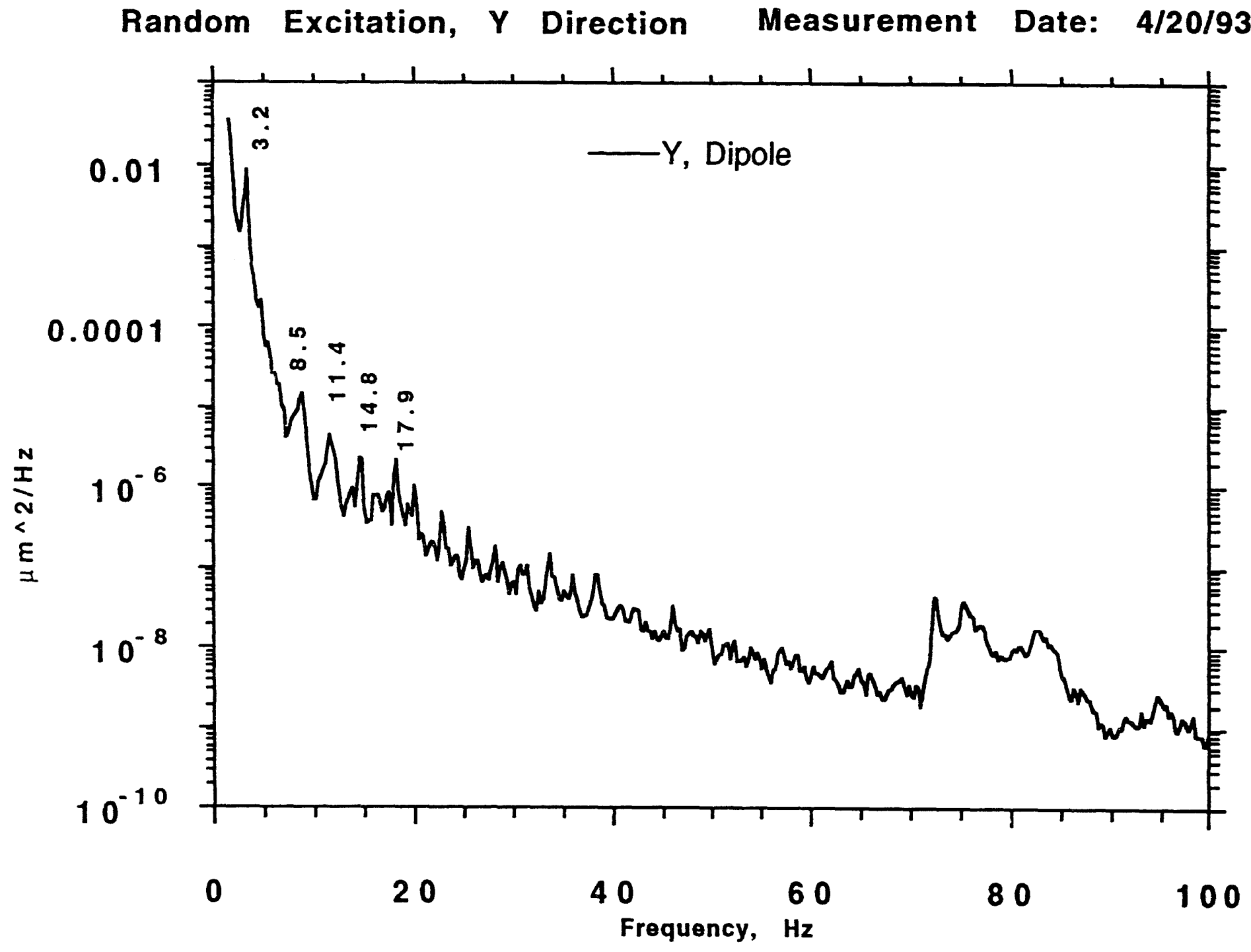




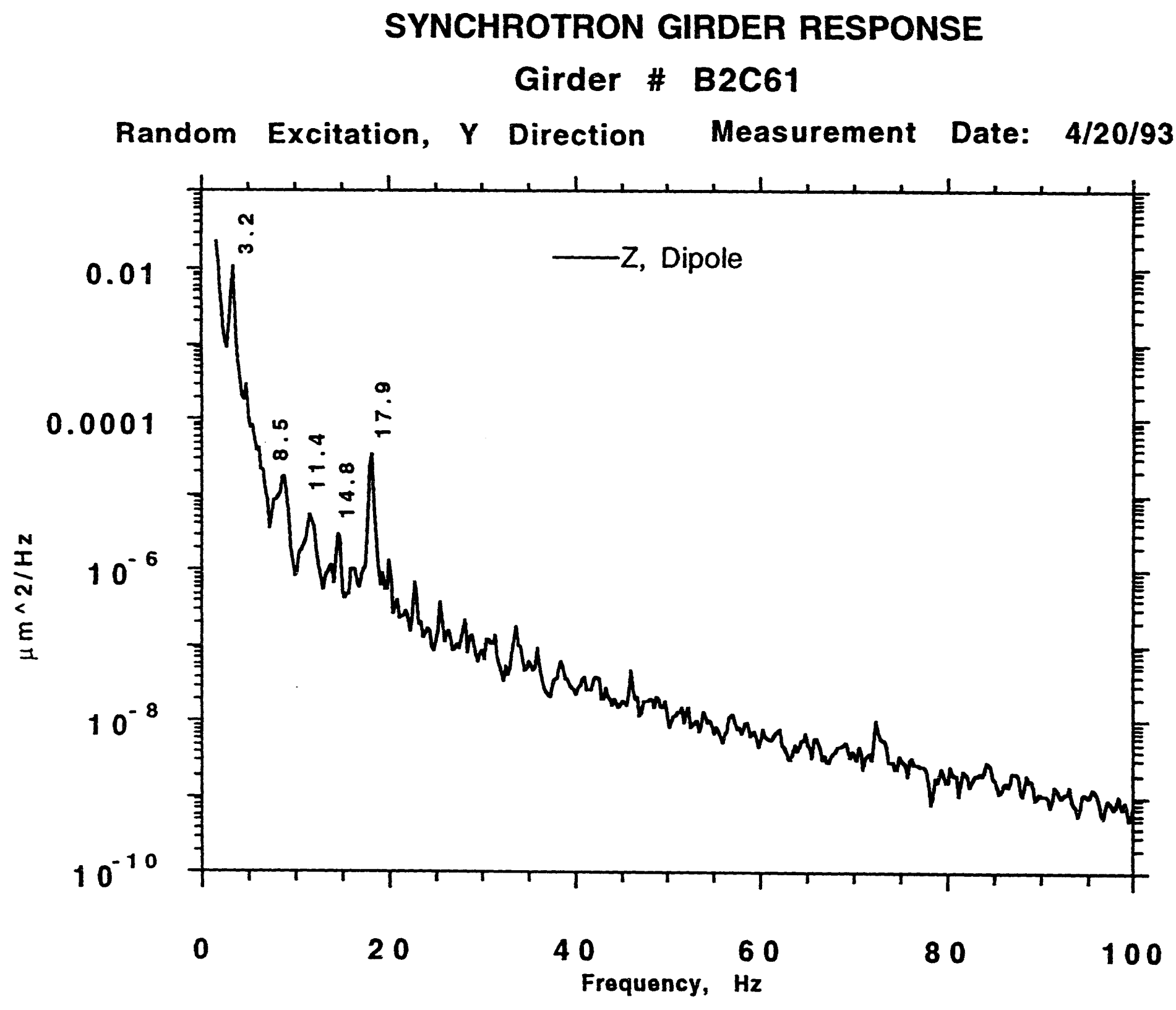




$$
\text { A-11 }
$$

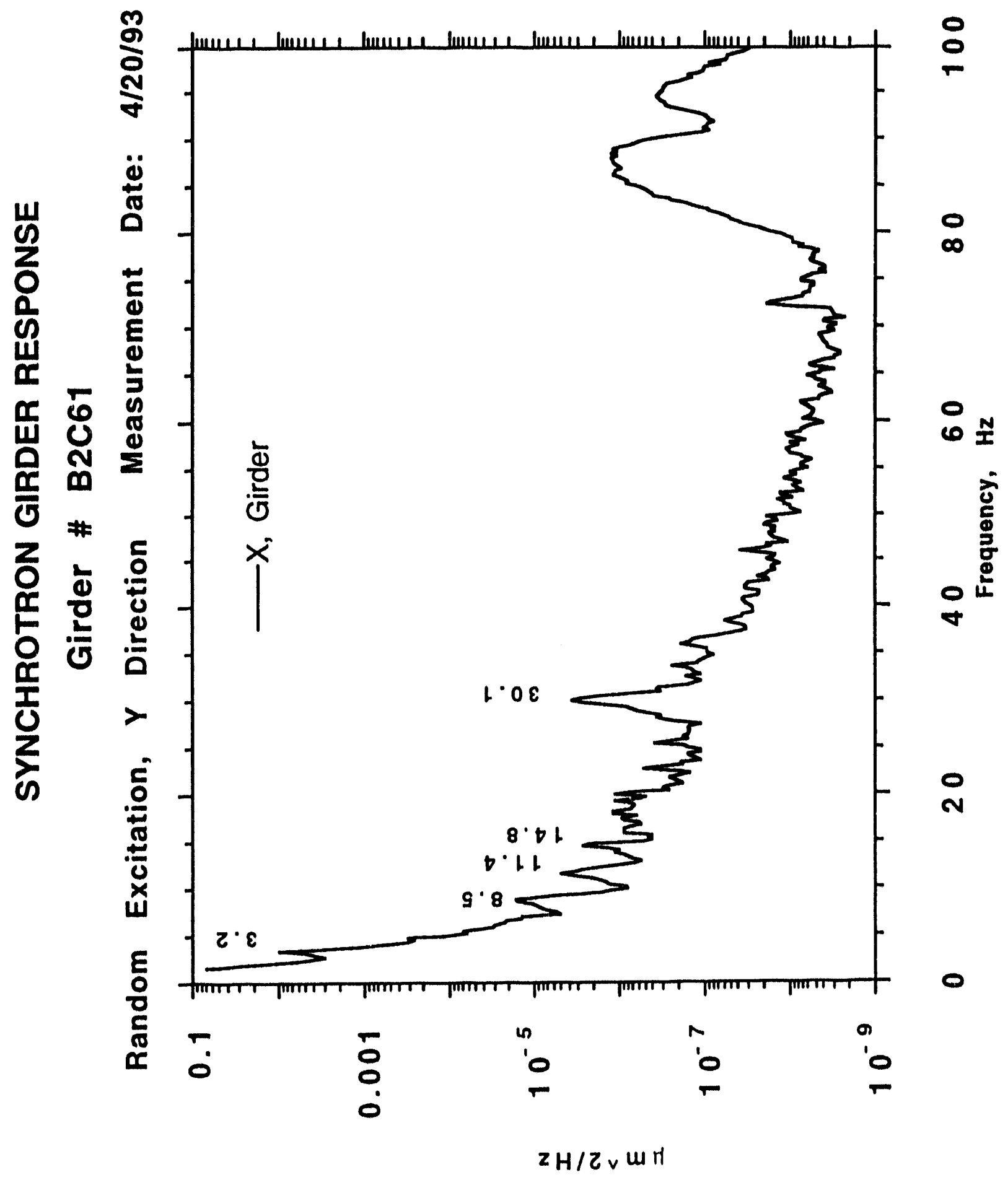




\section{SYNCHROTRON GIRDER RESPONSE}

Girder \# B2C61

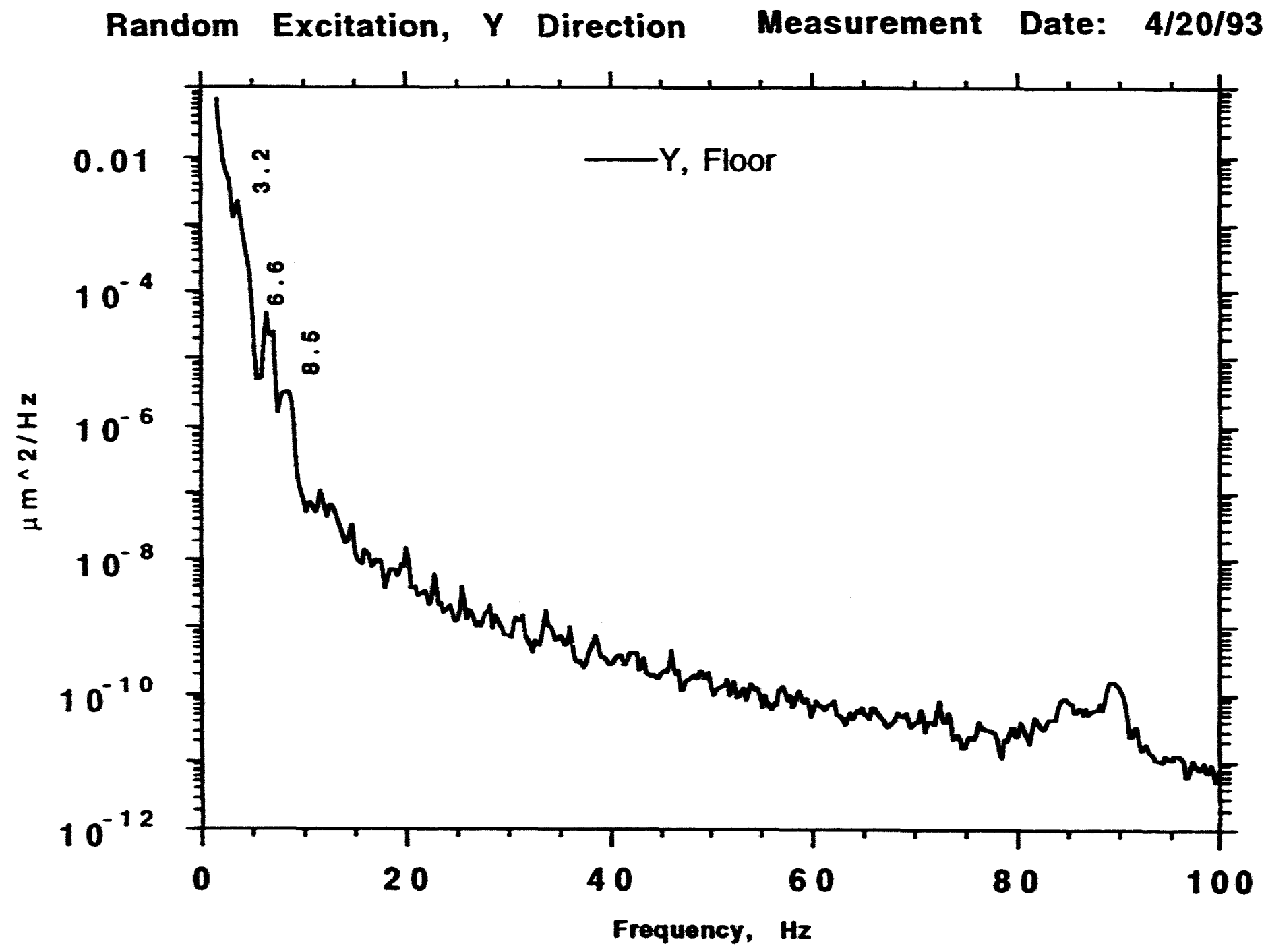




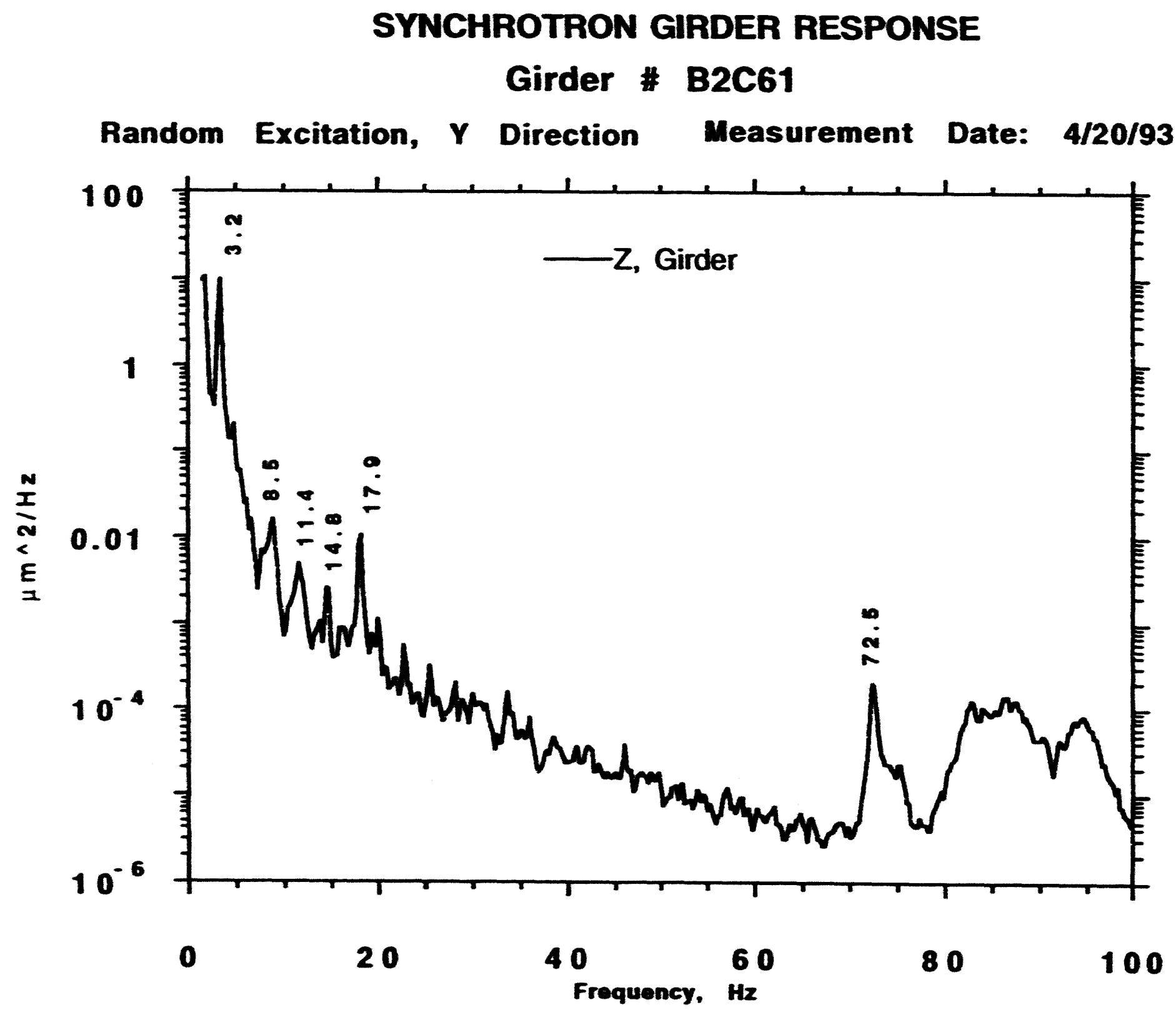




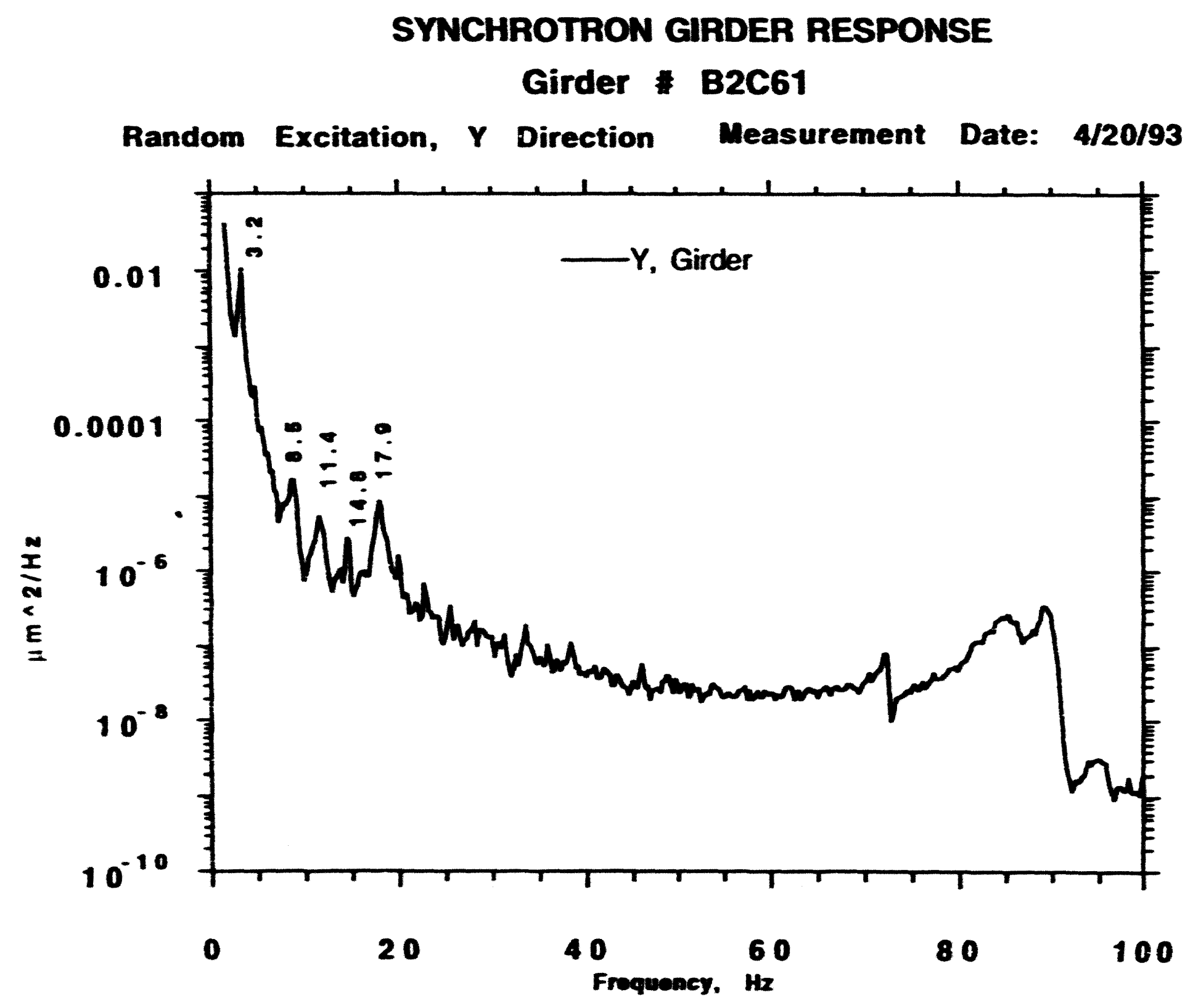




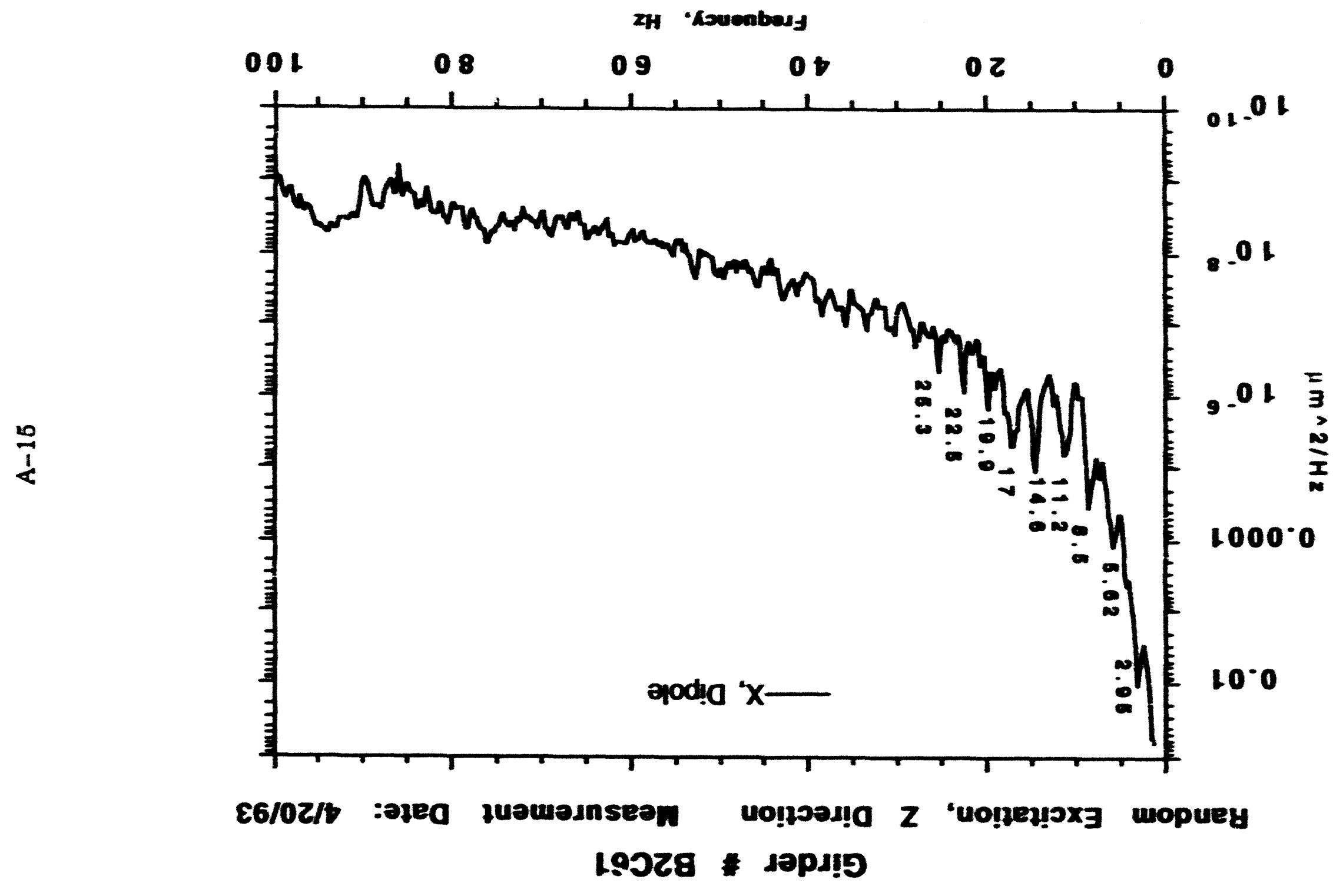

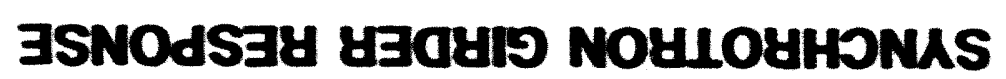




\section{SYNCHROTRON GIRDER RESPONSE}

Girder B2C61

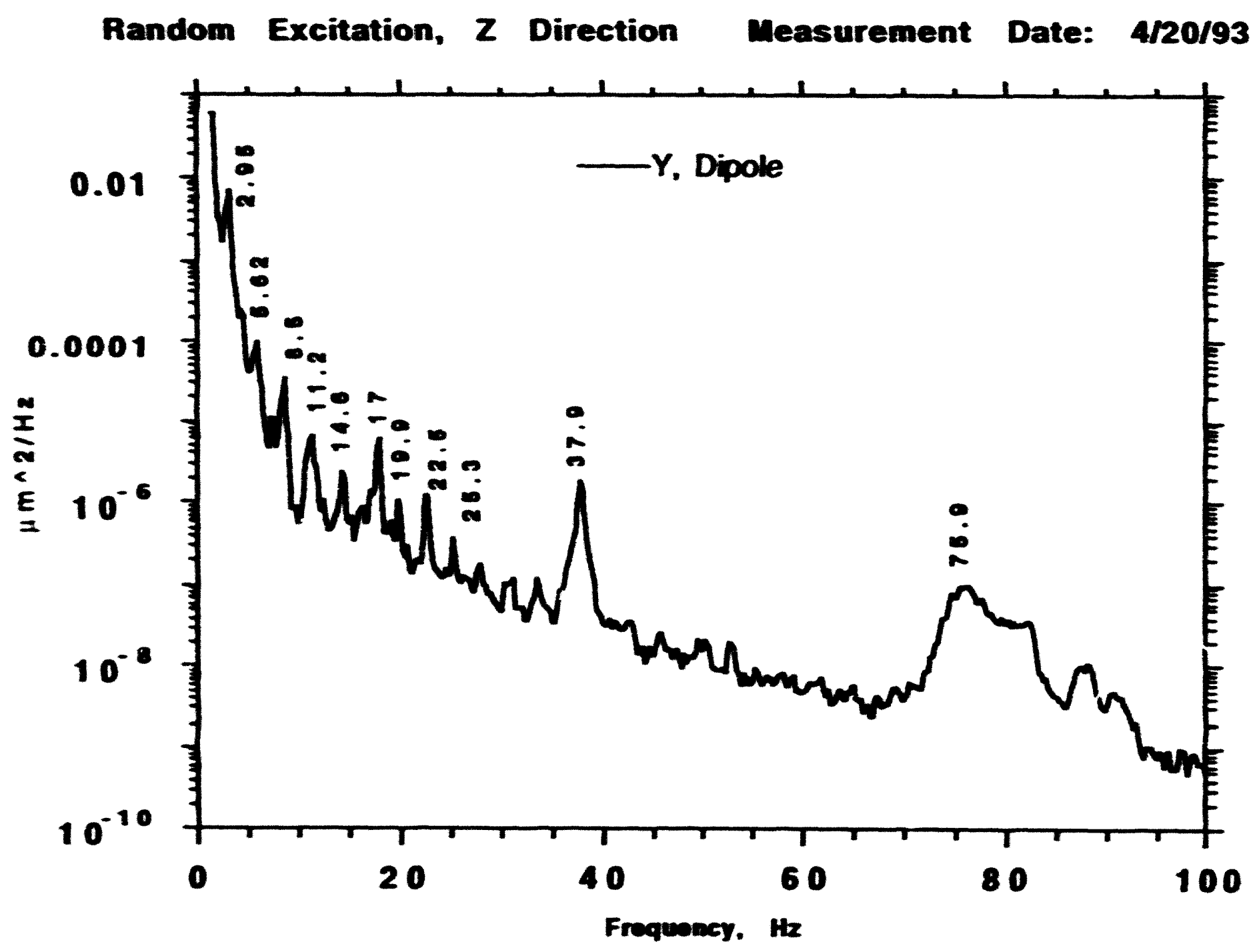

$\frac{1}{0}$ 


\section{SYNCHROTRON GIRDER RESPONSE}

Girder B2C61

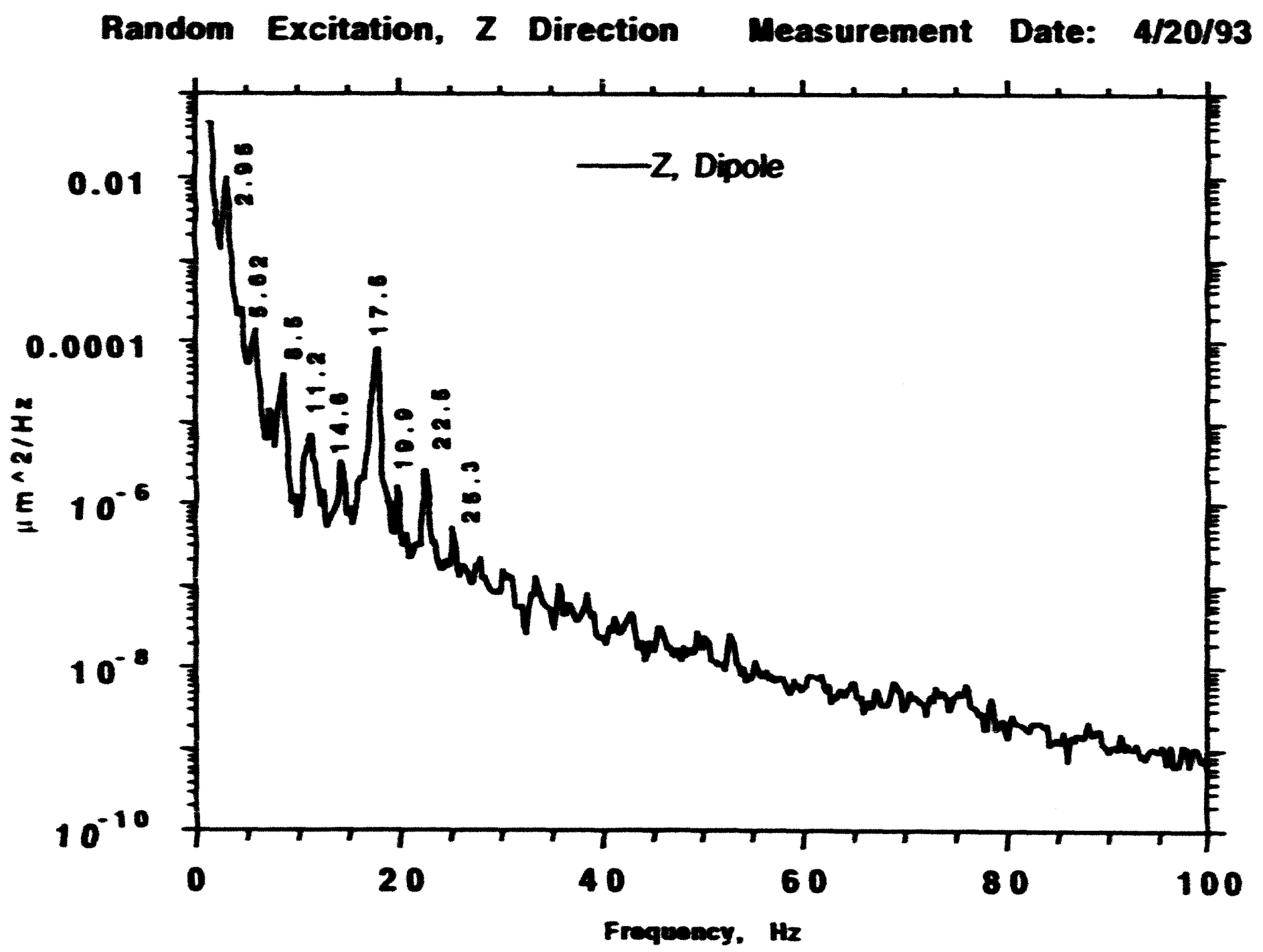




\section{SWNCHROTRON GIRDER RESPONSE}

Girder B2C61

Random Excitation, $Z$ Direction Measurement Date: 4/20/93

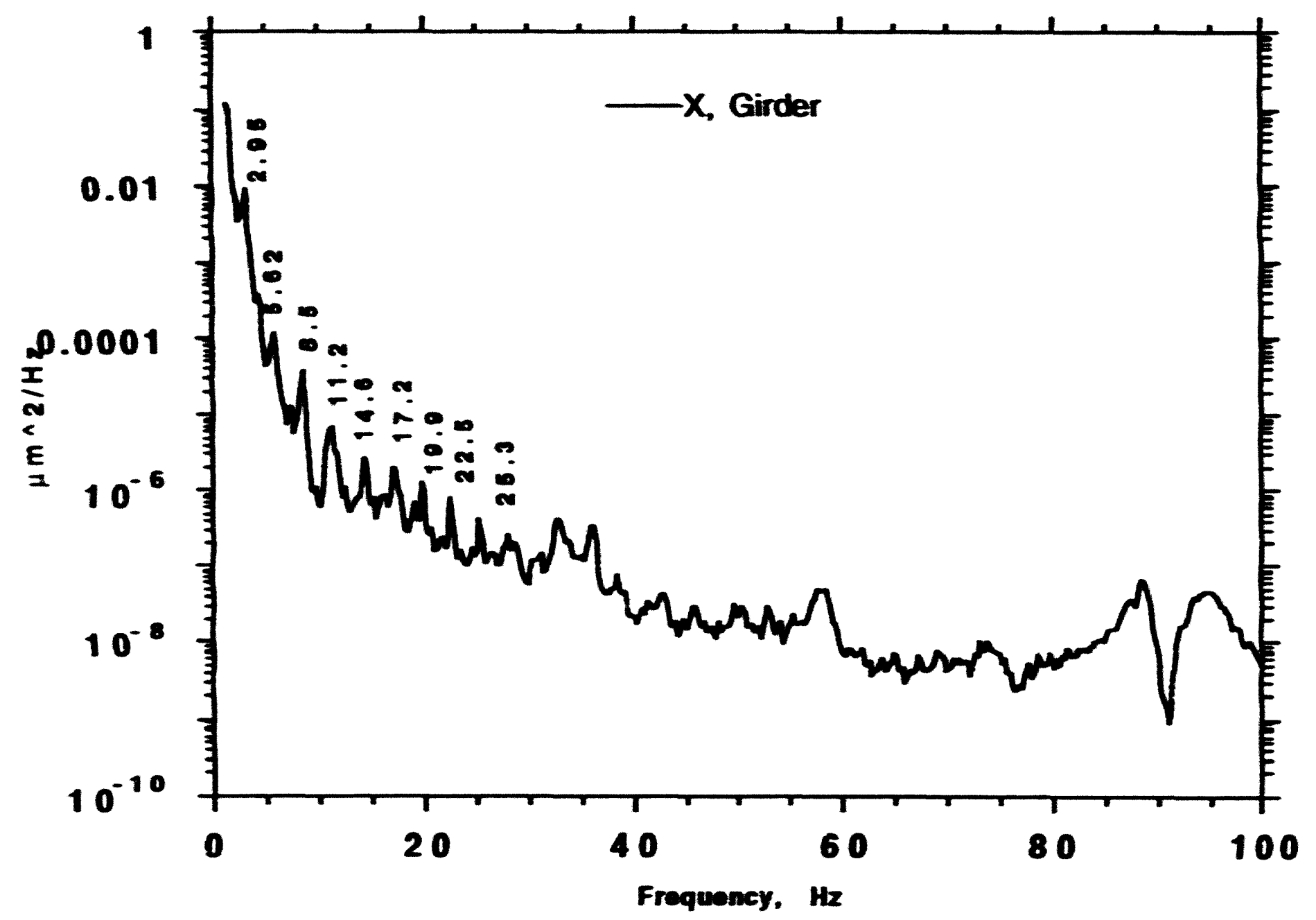




\section{SYNCHROTRON GIRDER RESPONSE}

Girder \# B2C61

Random Excitation, $Z$ Direction Measurement Date: 4/20/93

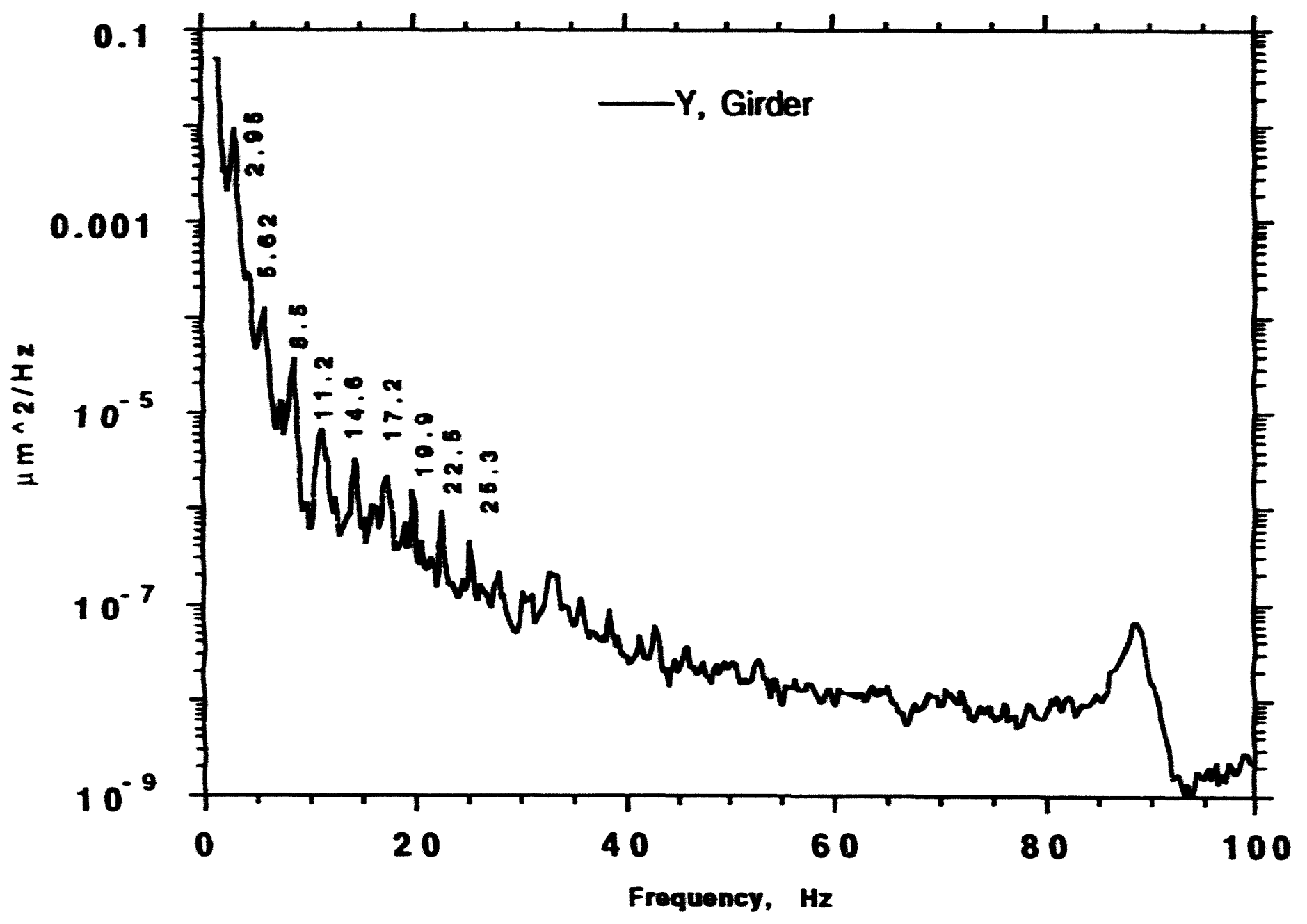




\section{SYNCHROTRON GIRDER RESPONSE}

Girder \# B2C61

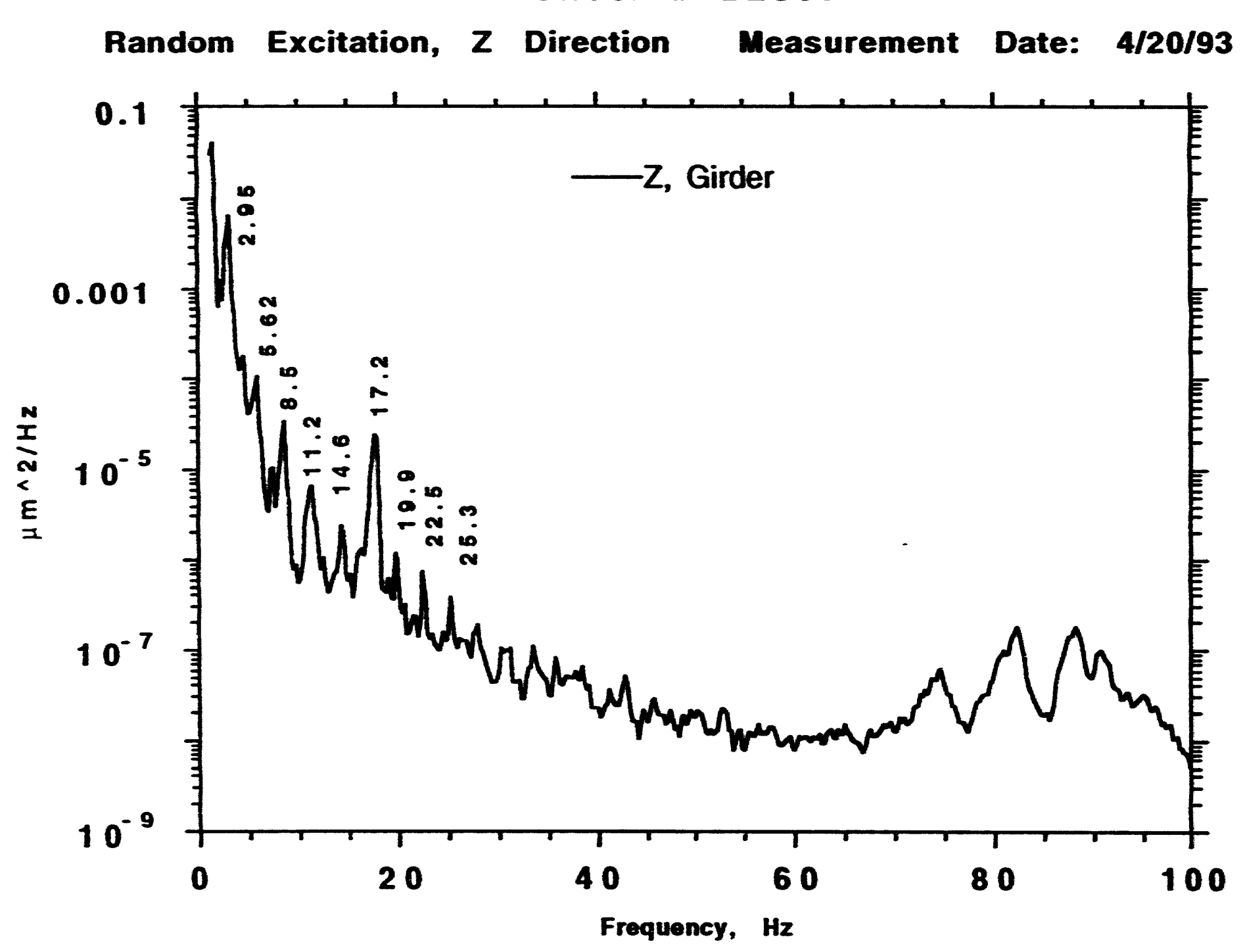




\section{SYNCHROTRON GIRDER RESPONSE}

Girder \# B2C61

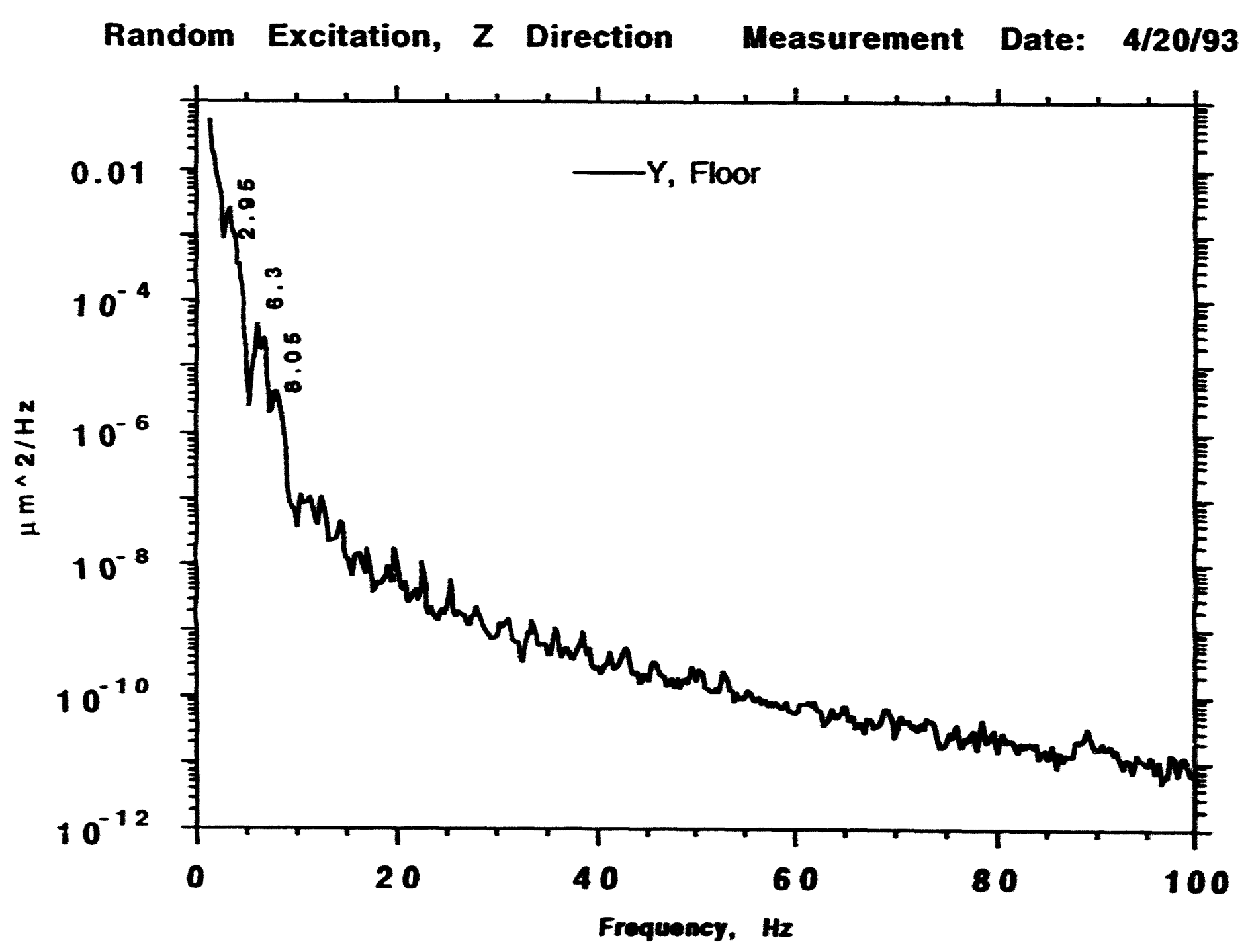




\section{SYNCHROTRON GIRDER RESPONSE}

Girder \# B2C61

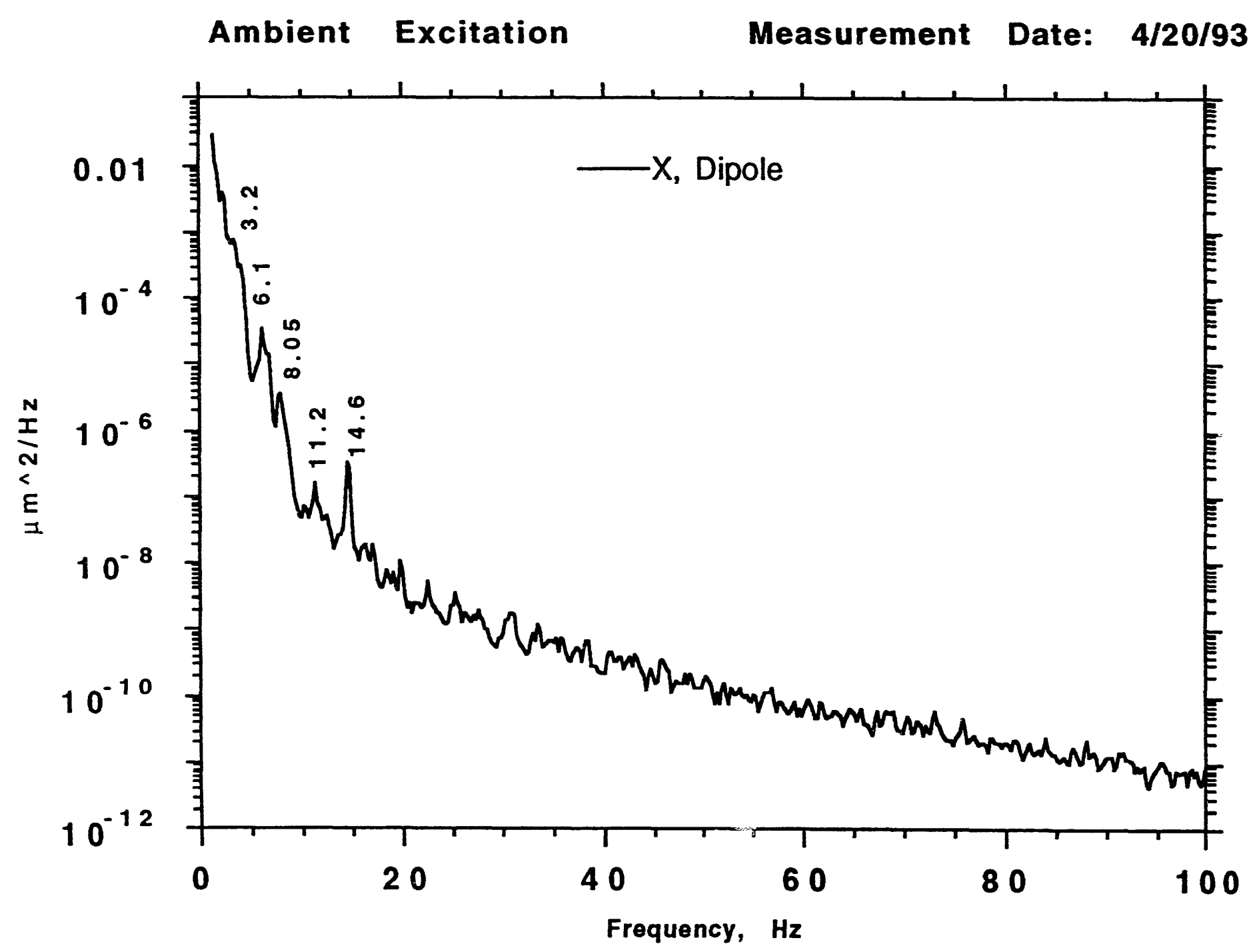


SYNCHROTRON GIRDER RESPONSE

Girder \# B2C61

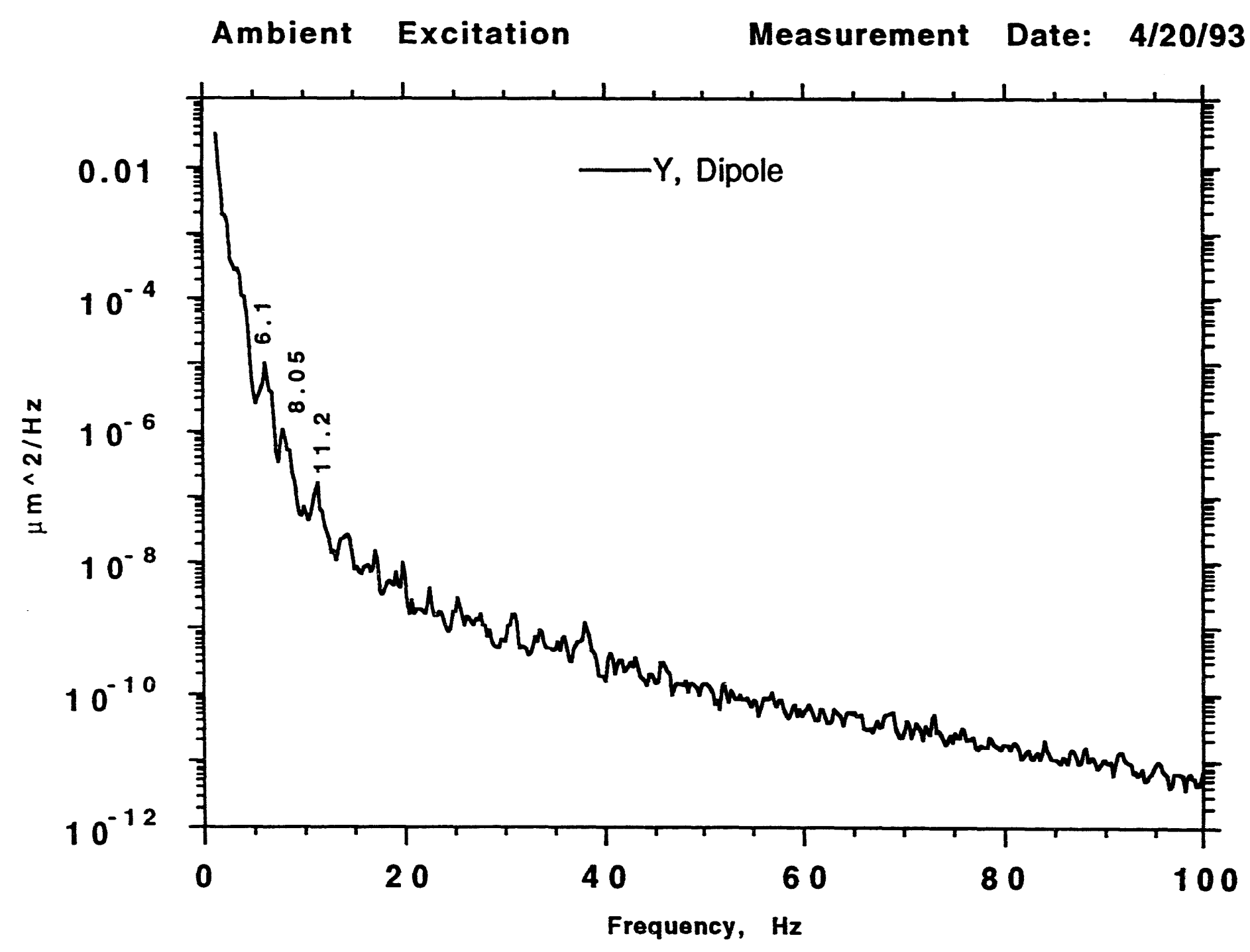


SYNCHROTRON GIRDER RESPONSE

Girder \# B2C61

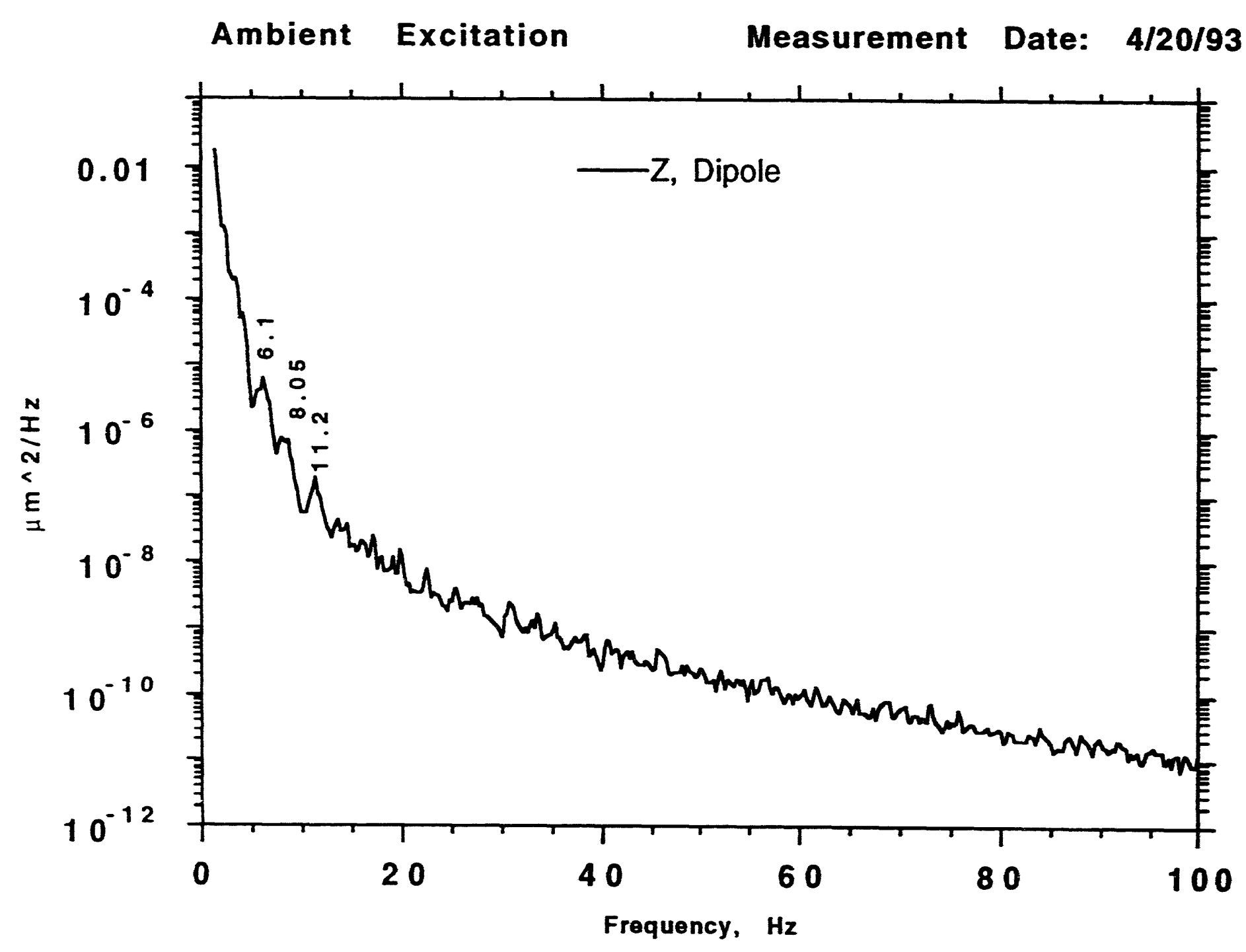




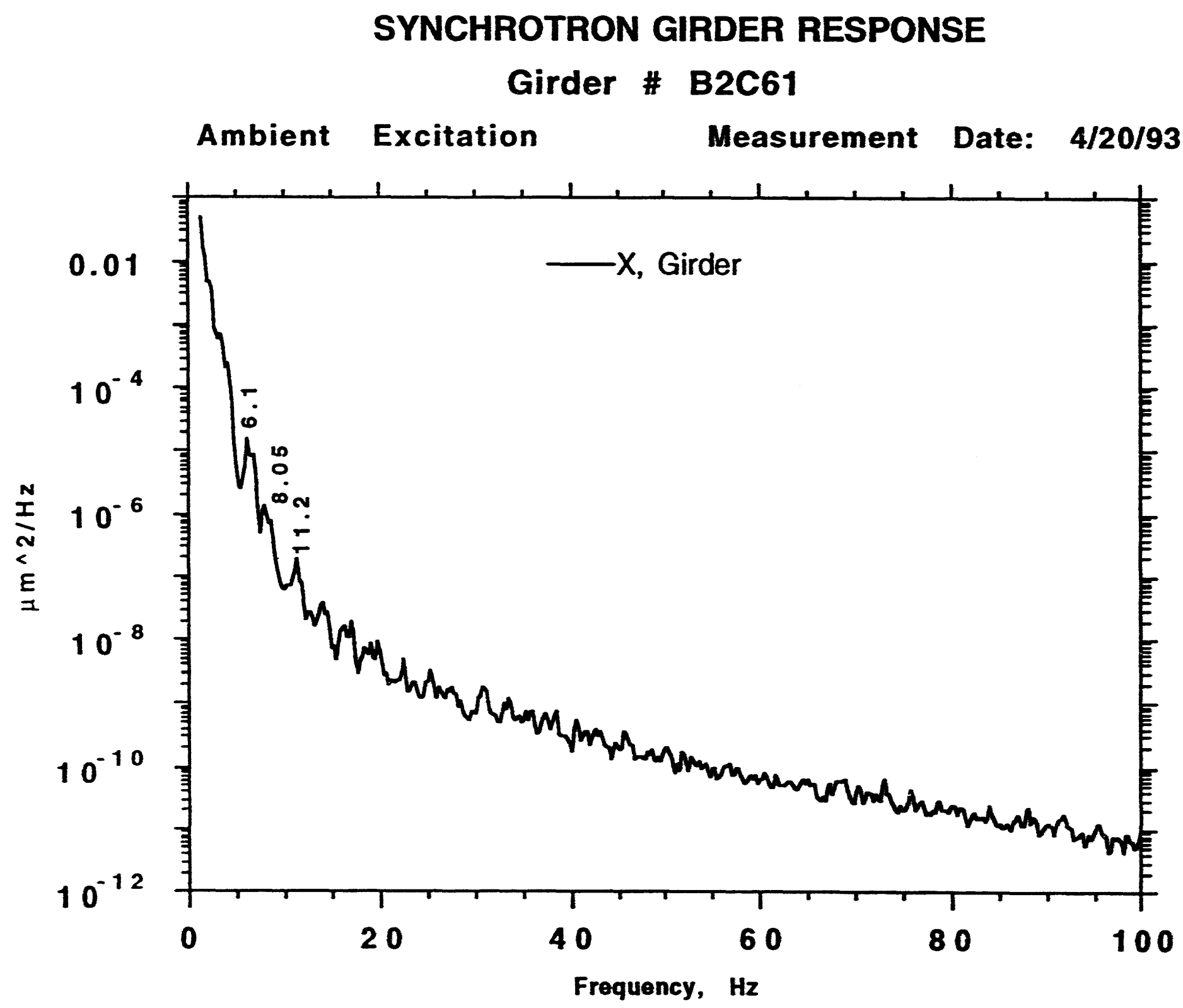




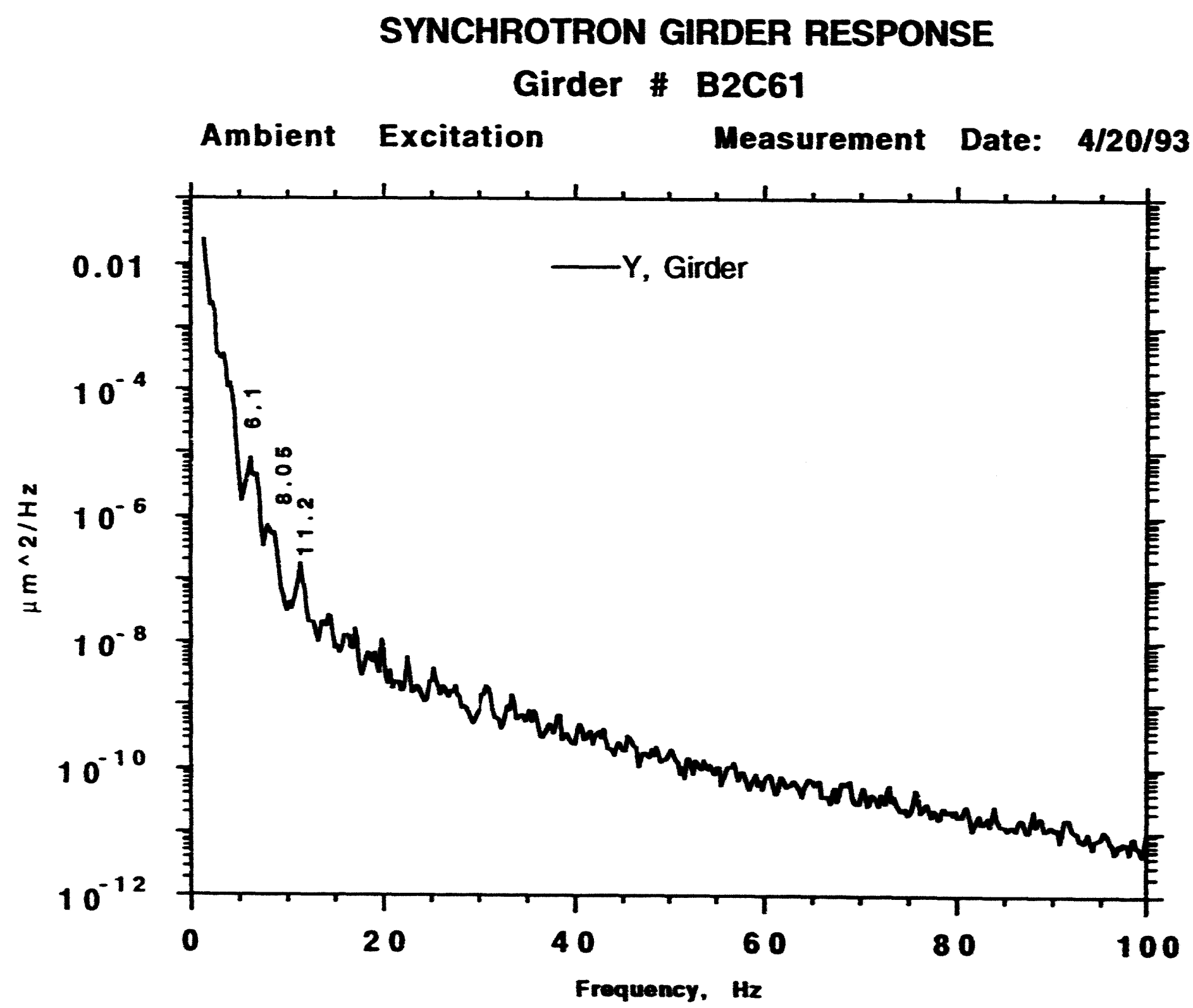




\section{SYNCHROTRON GIRDER RESPONSE}

\section{Girder B2C61}

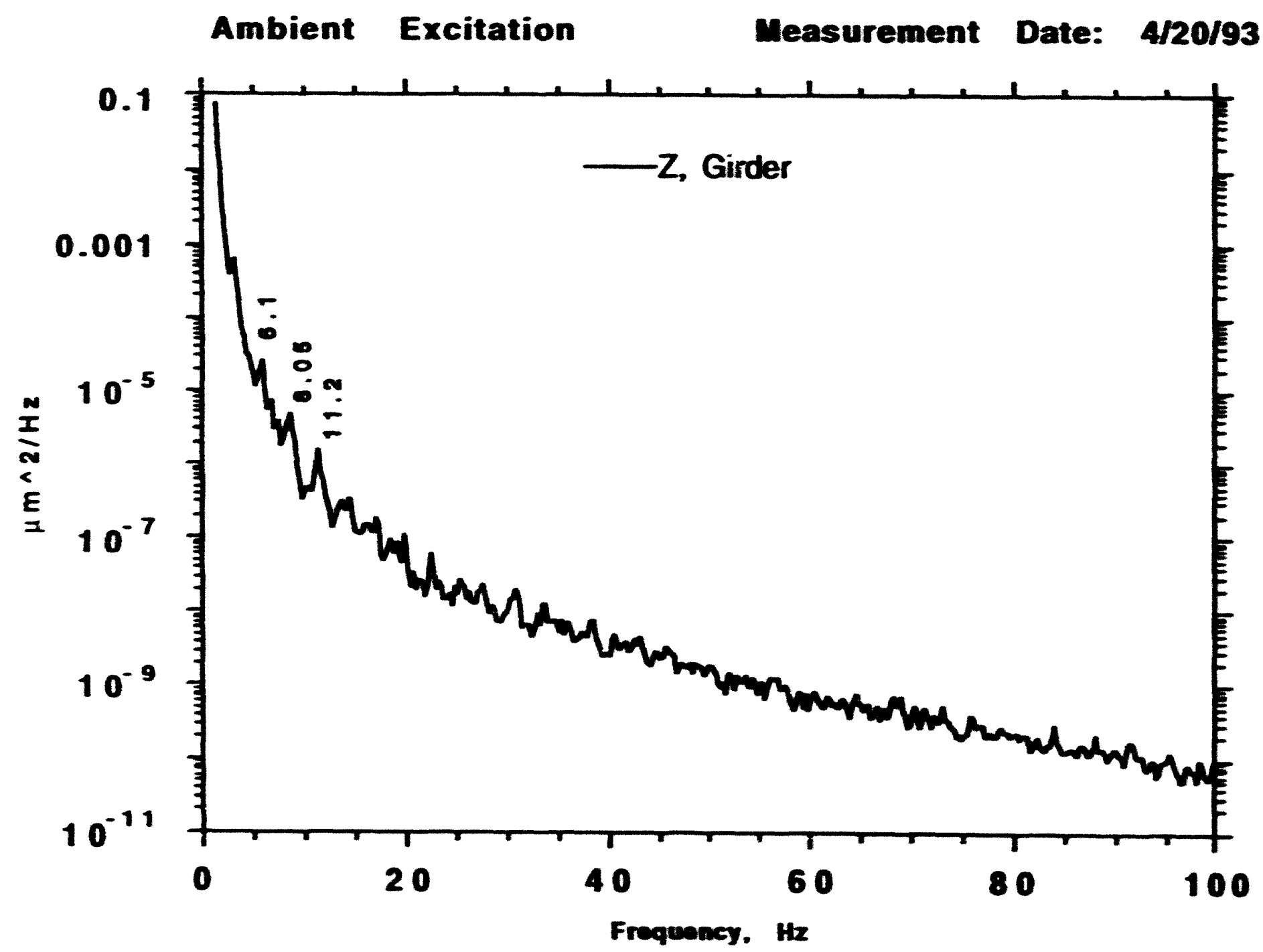


SYNCHROTRON GIRDER RESPONSE

Girder * B2C61

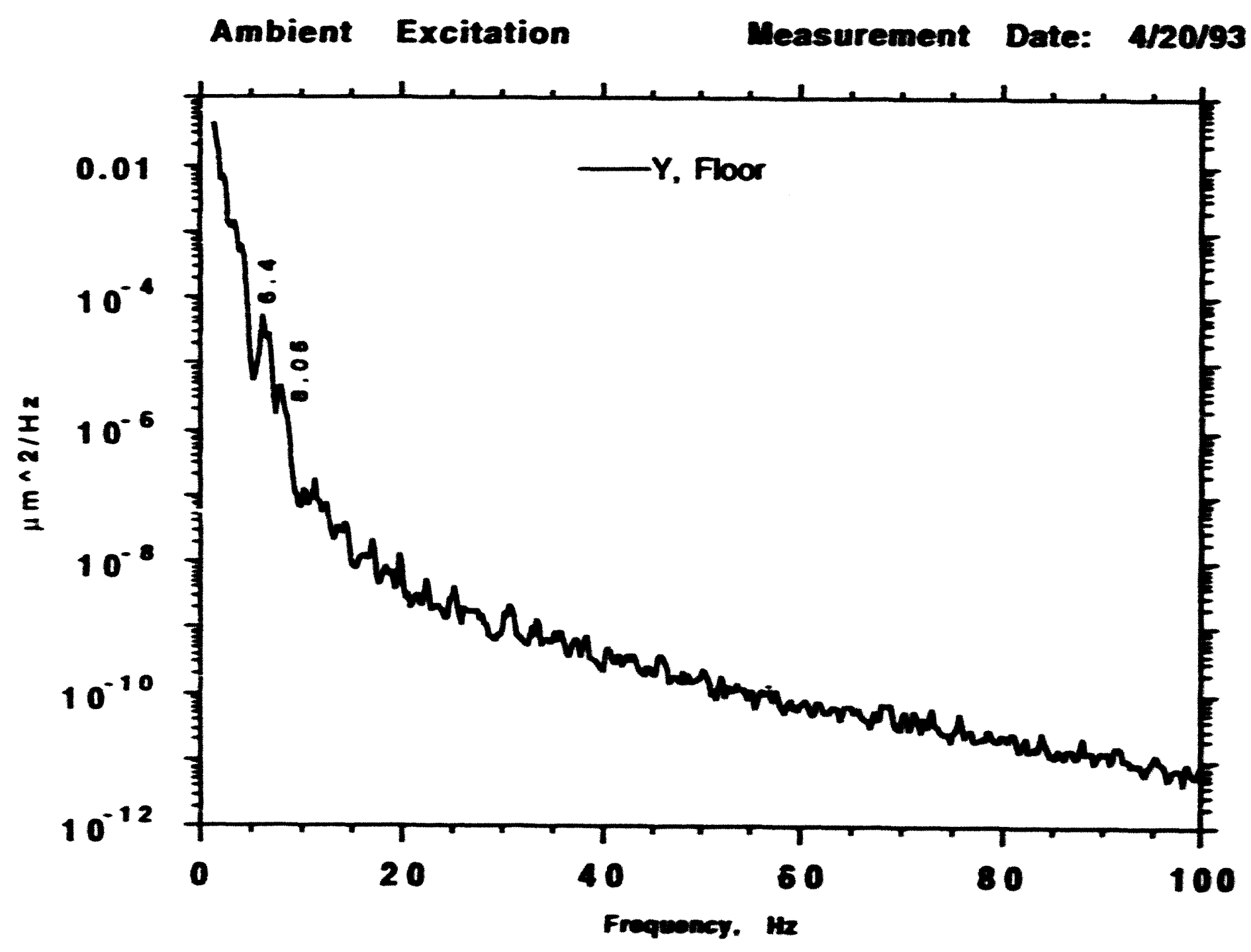




\section{Appendix Bi Horizontal correotor-magnet reoponse}


B-1

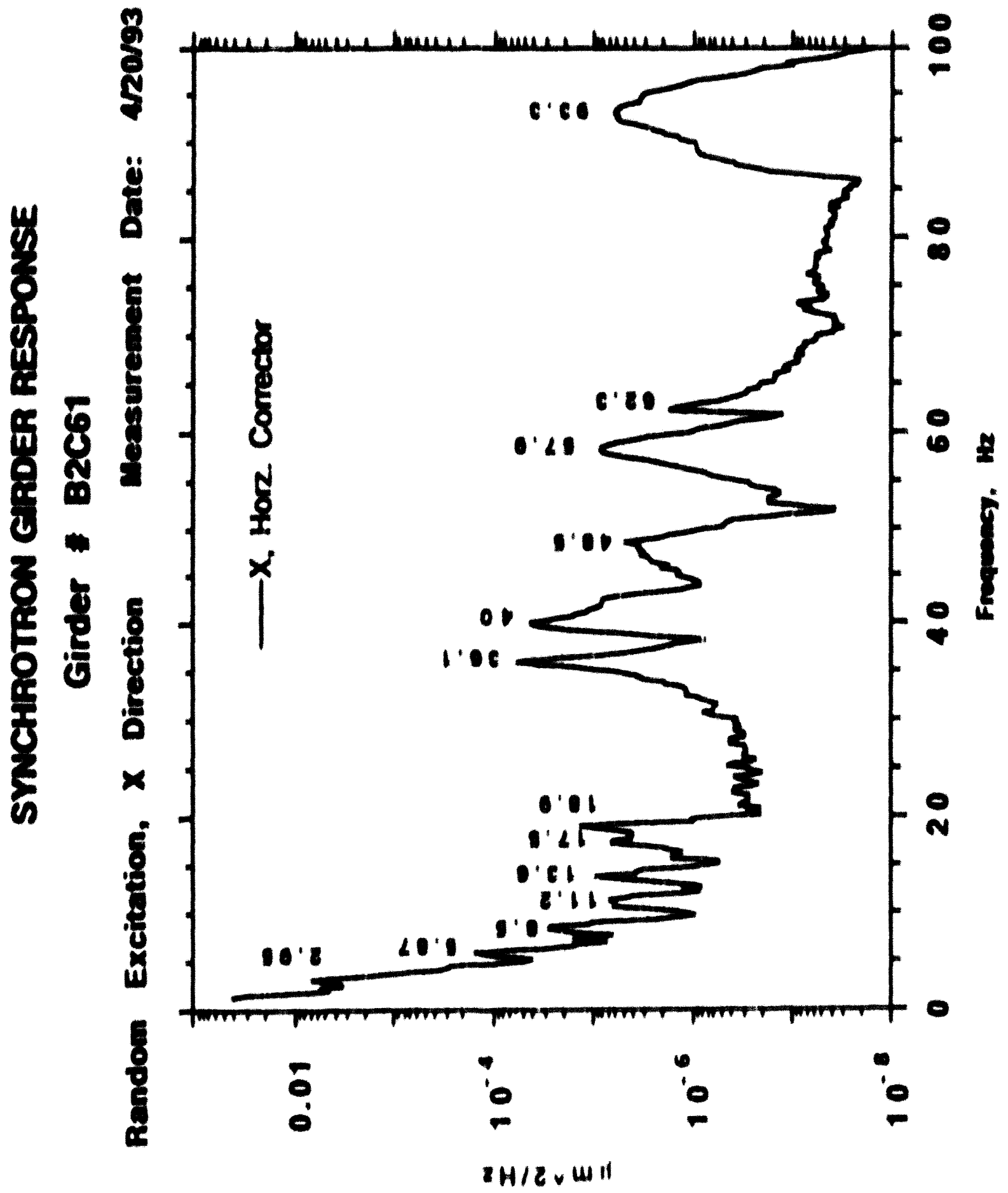




\section{SYNCHROTRON GRDER RESPONSE}

\section{Girder B2C61}

Random Excitation, $X$ Direction Measurement Date: 4/20/93

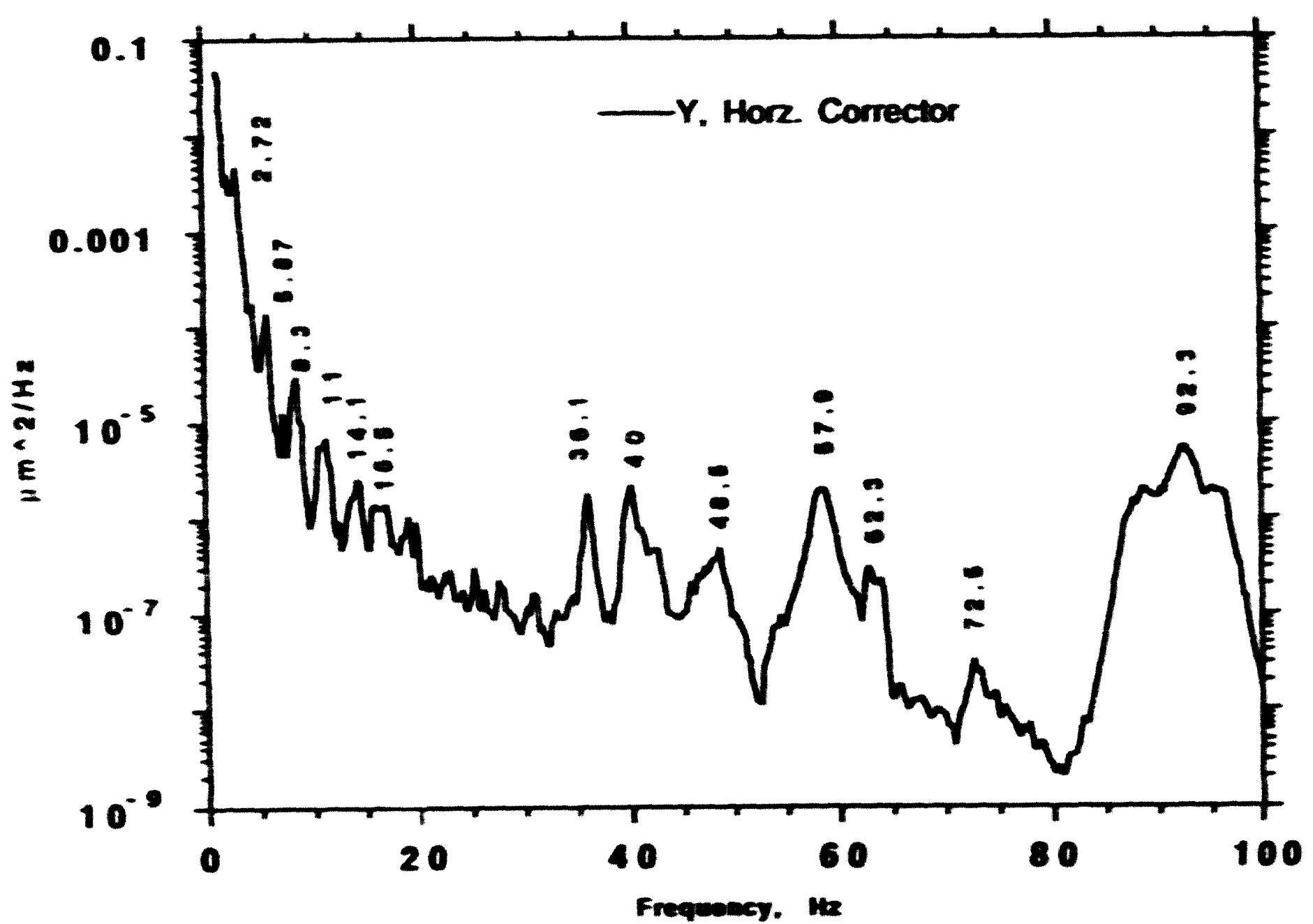

I 


\section{SYNCHROTHON GIRDER RESPOUSE}

Girder B2C61

Random Excitation, X Direction Measurement Date: 4/20/93

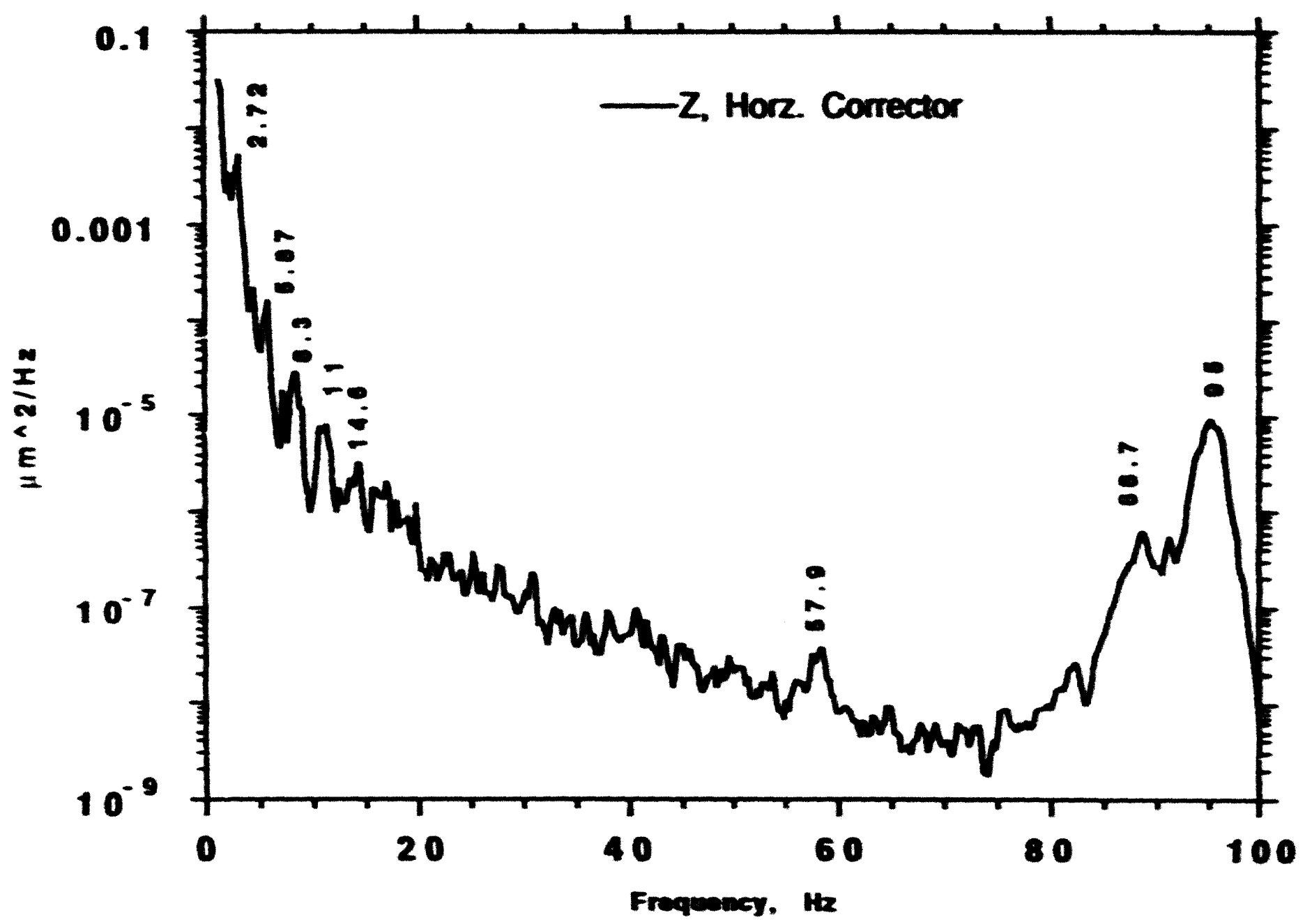

点 


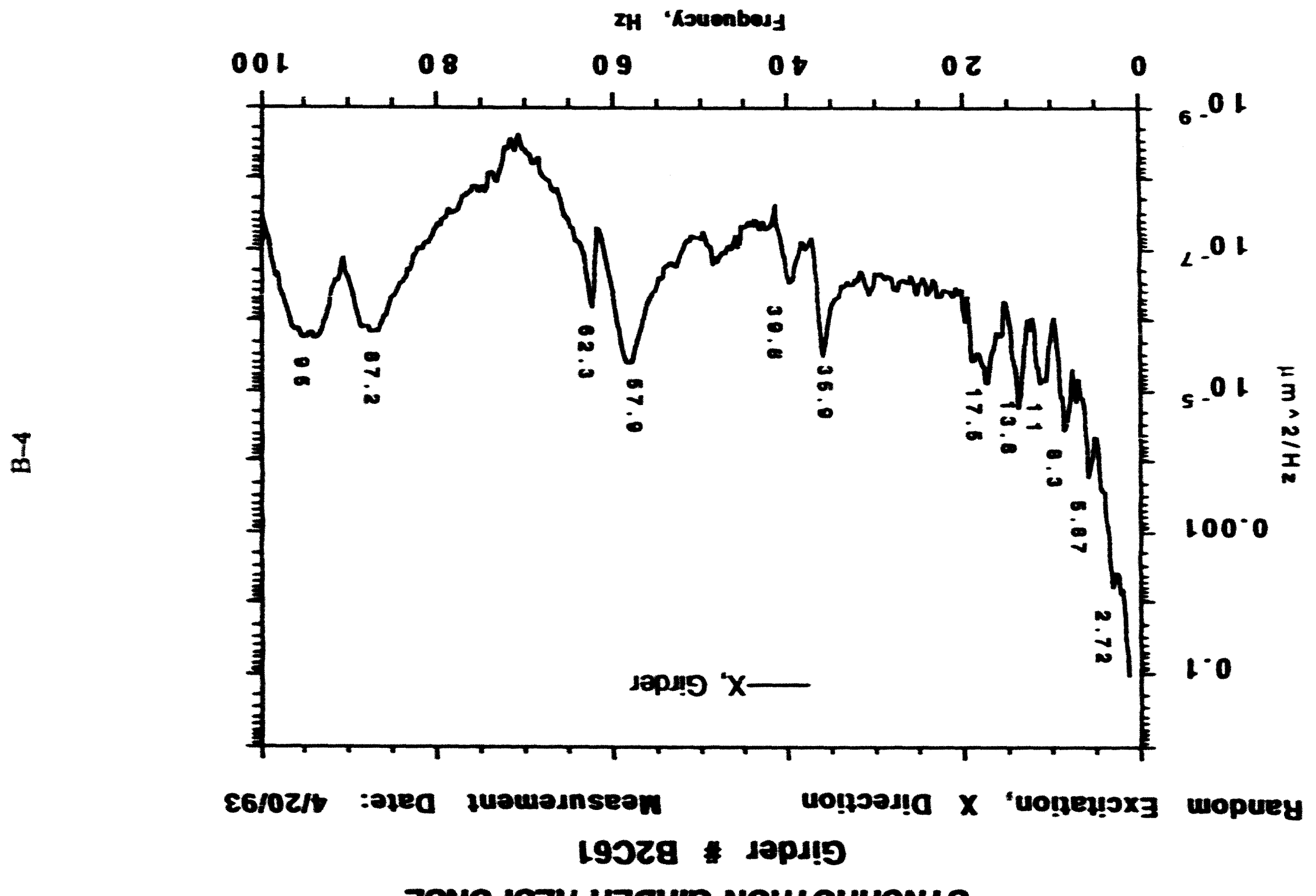

3SNOdSzY y 
SYNCHROTRON GIRDER RESPONSE

Girder B2C61

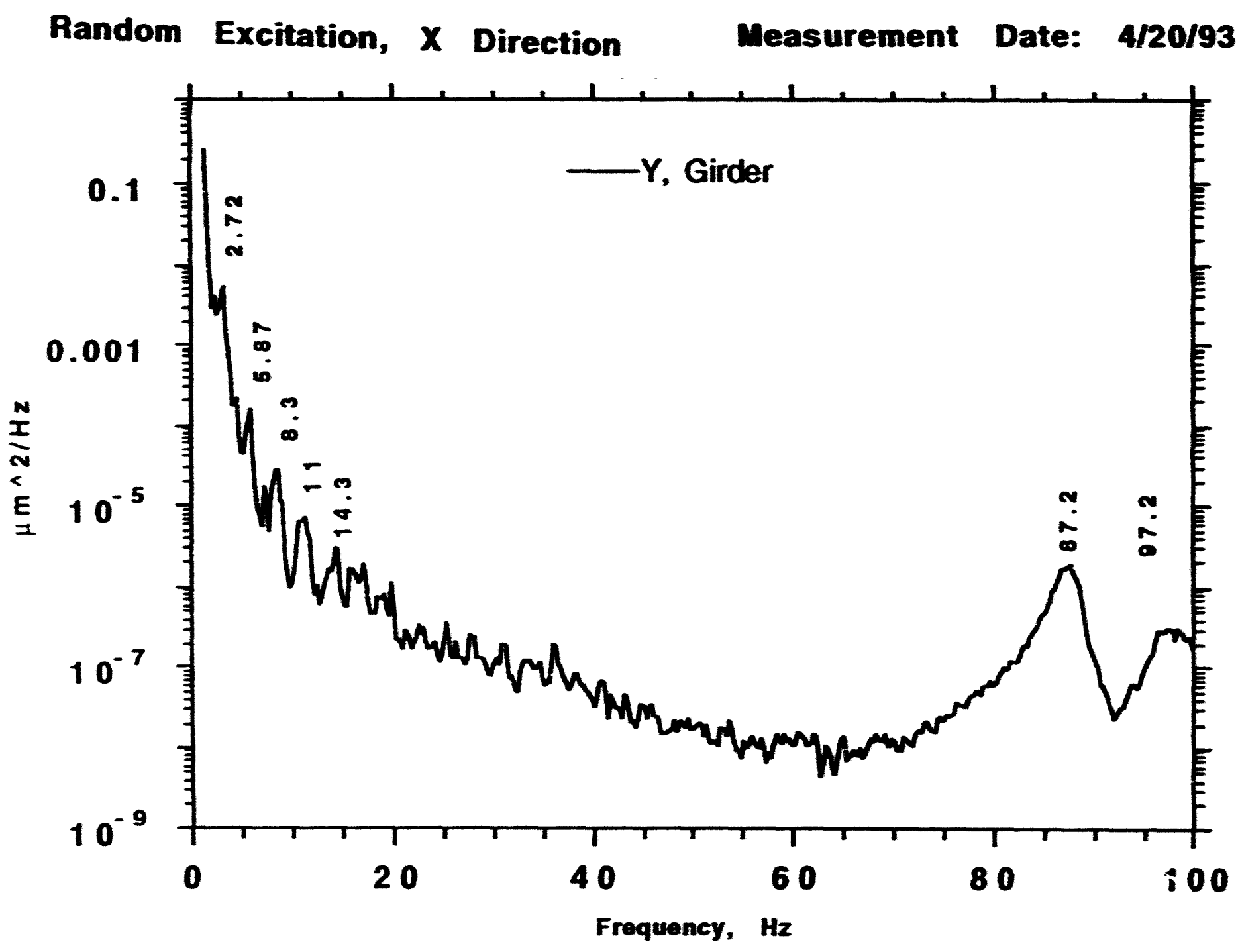




\section{SYNCHROTRON GIRDER RESPONSE}

Girder \# B2C61

Random Excitation, $X$ Direction Measurement Date: 4/20/93

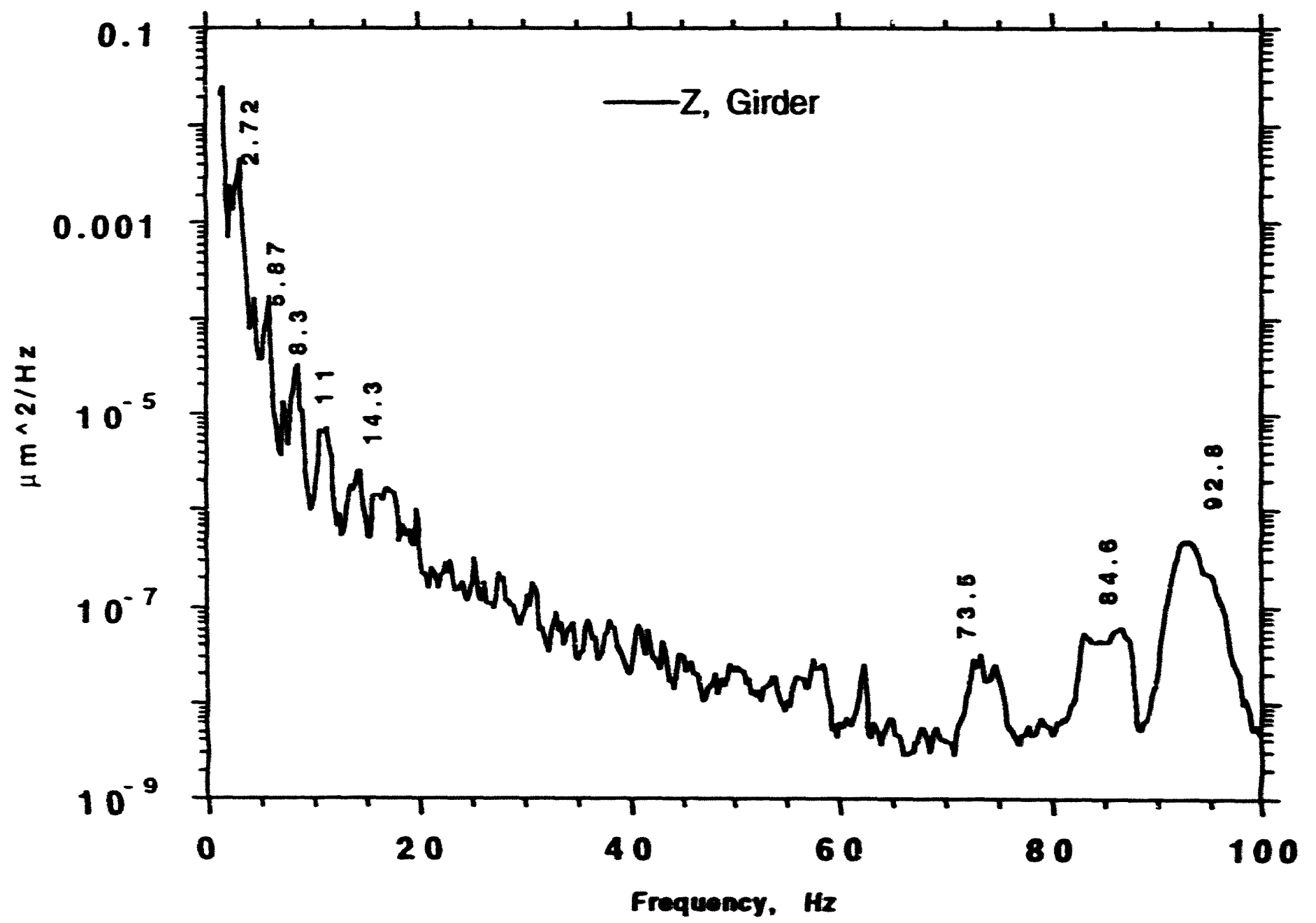




\section{SYNCHROTRON GIRDER RESPONSE}

\section{Girder \# B2C61}

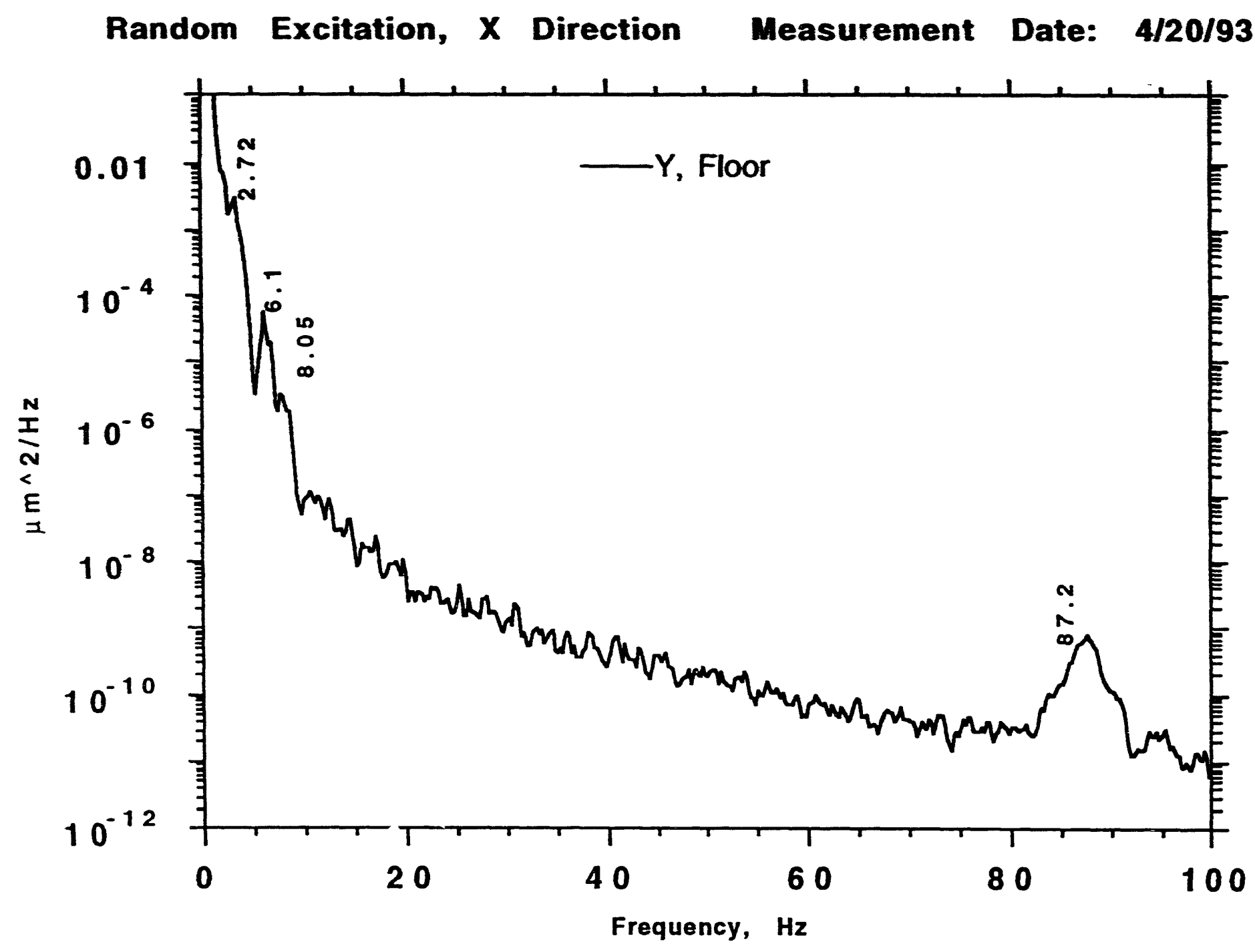


B- 8

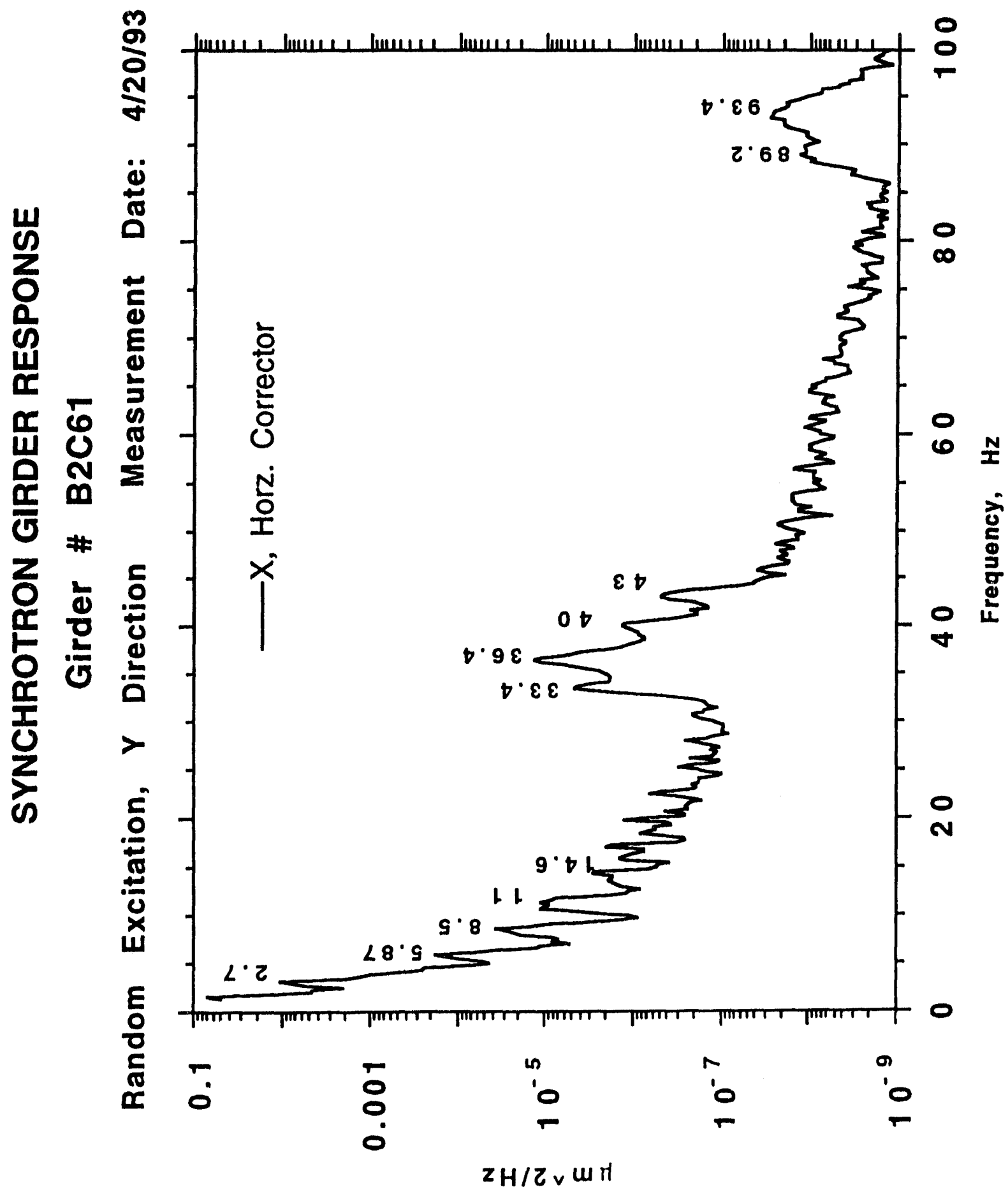




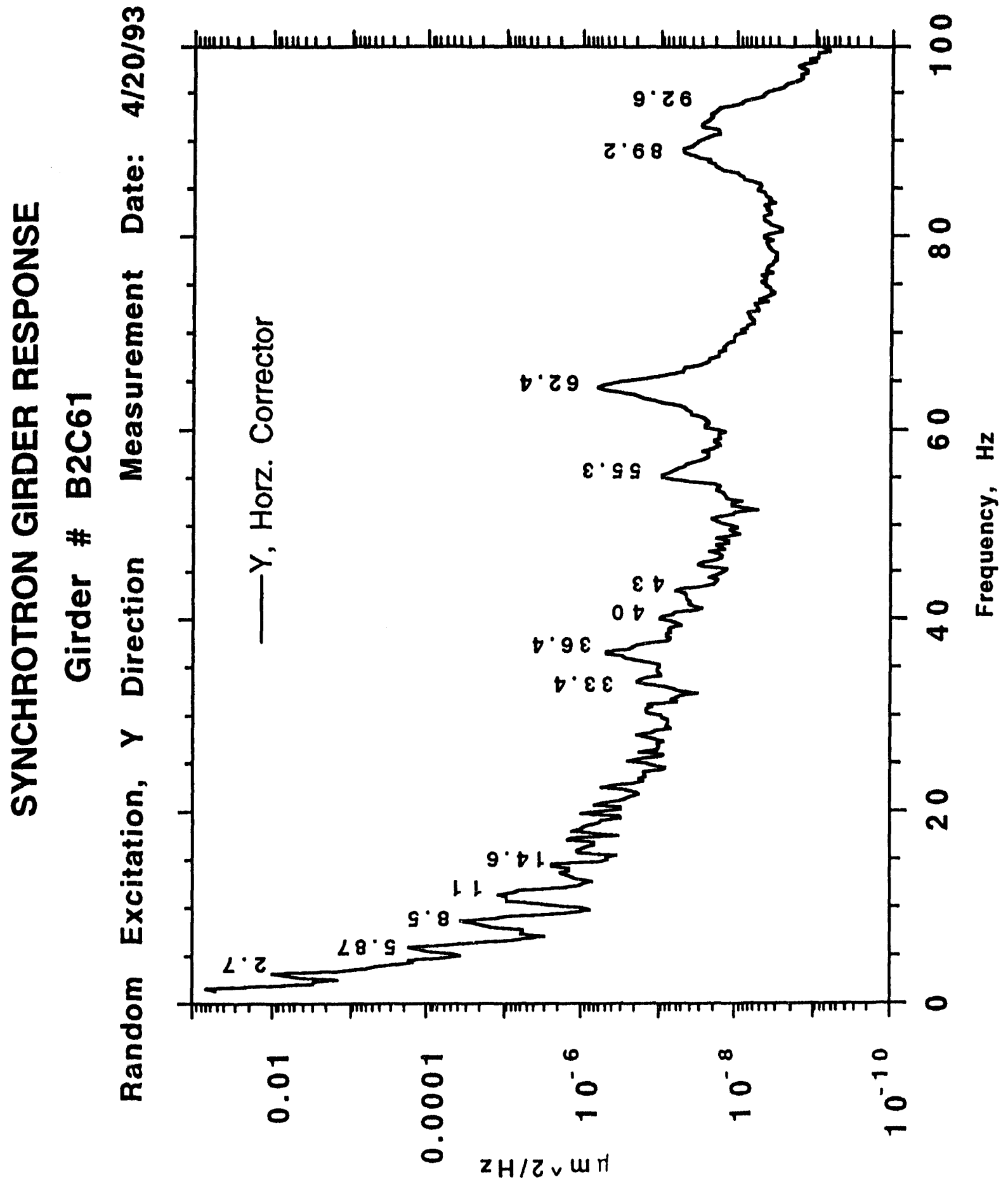




\section{SYNCHROTRON GIRDER RESPONSE}

Girder \# B2C61

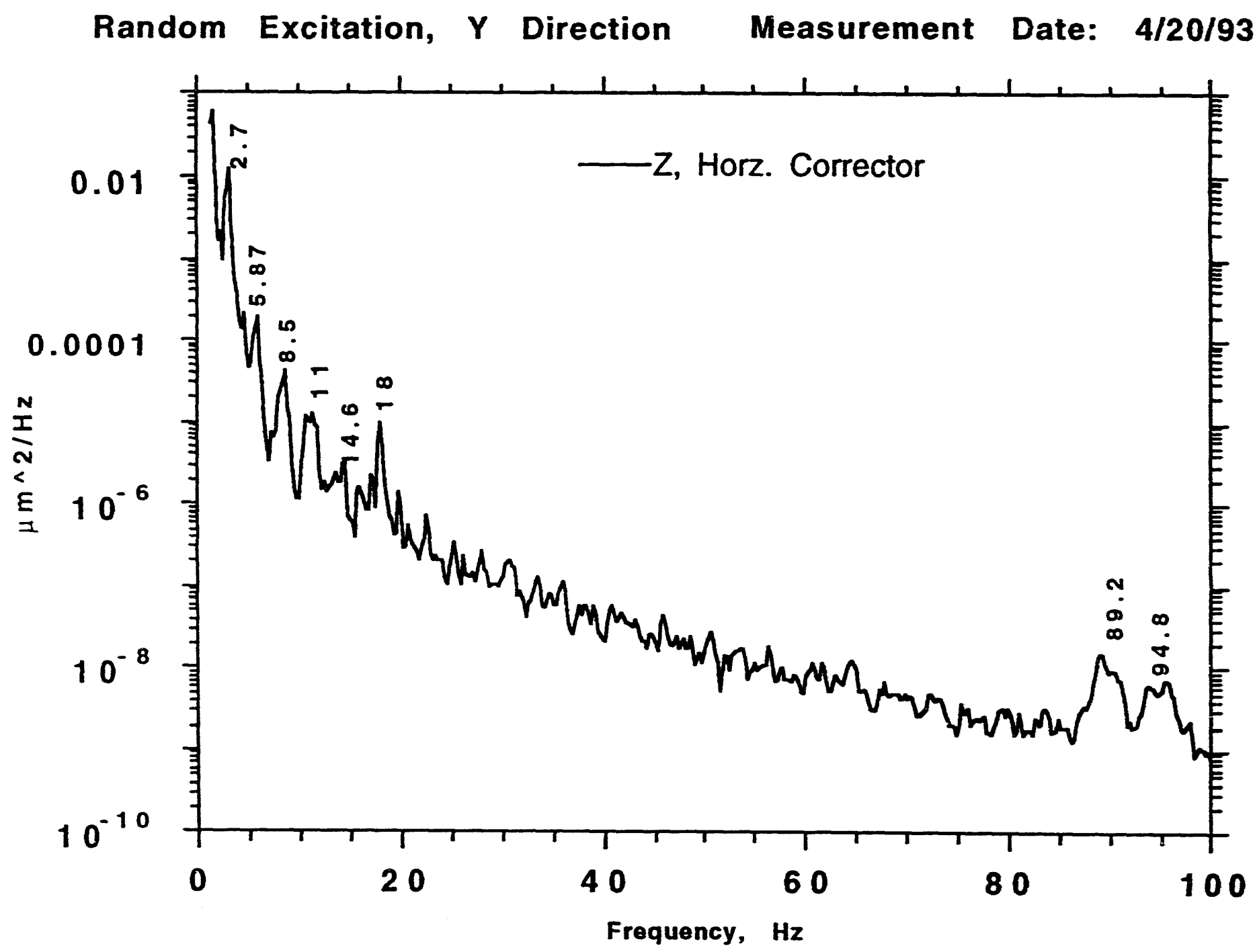




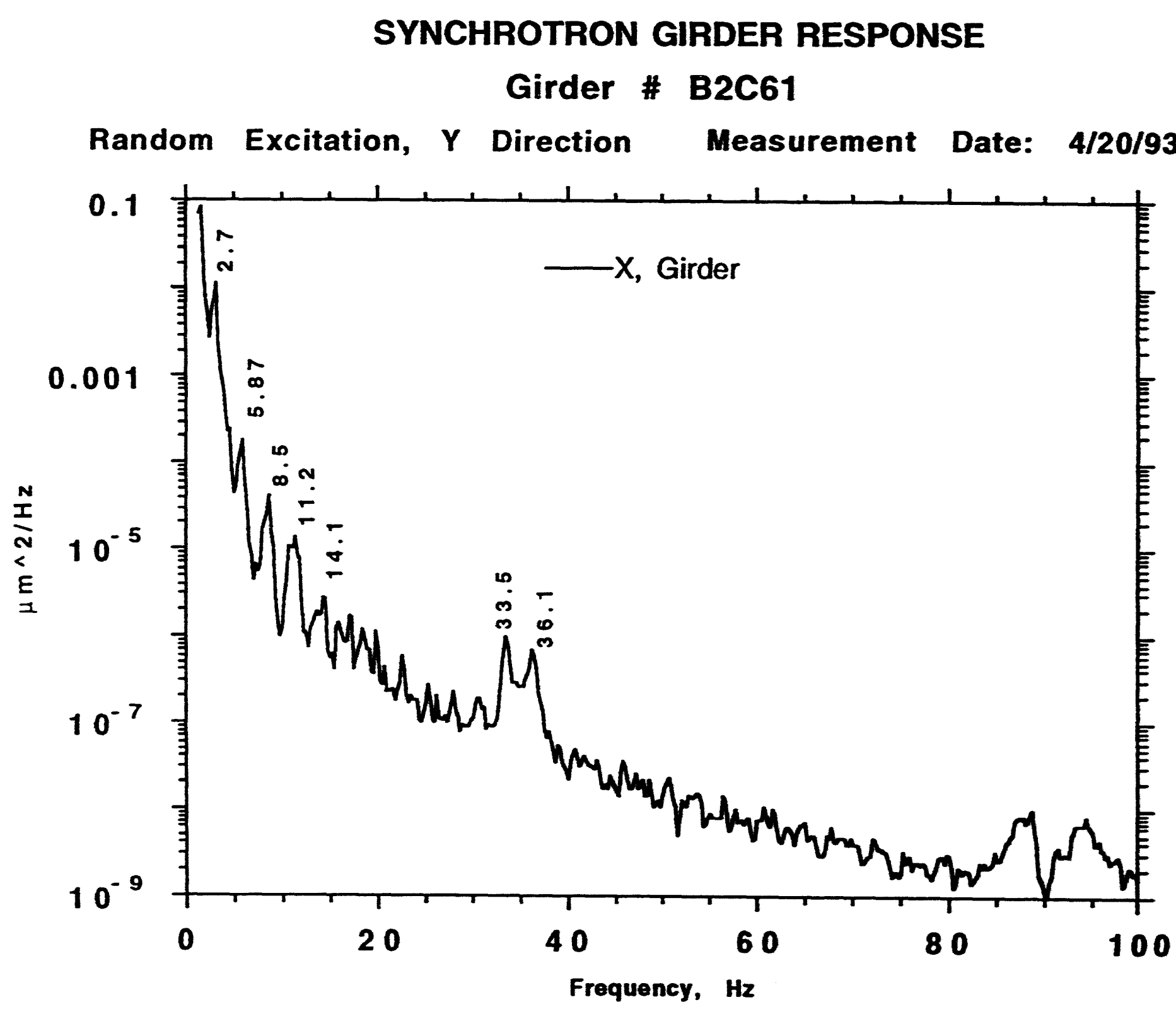




\section{SYNCHROTRON GIRDER RESPONSE}

\section{Girder \# B2C61}

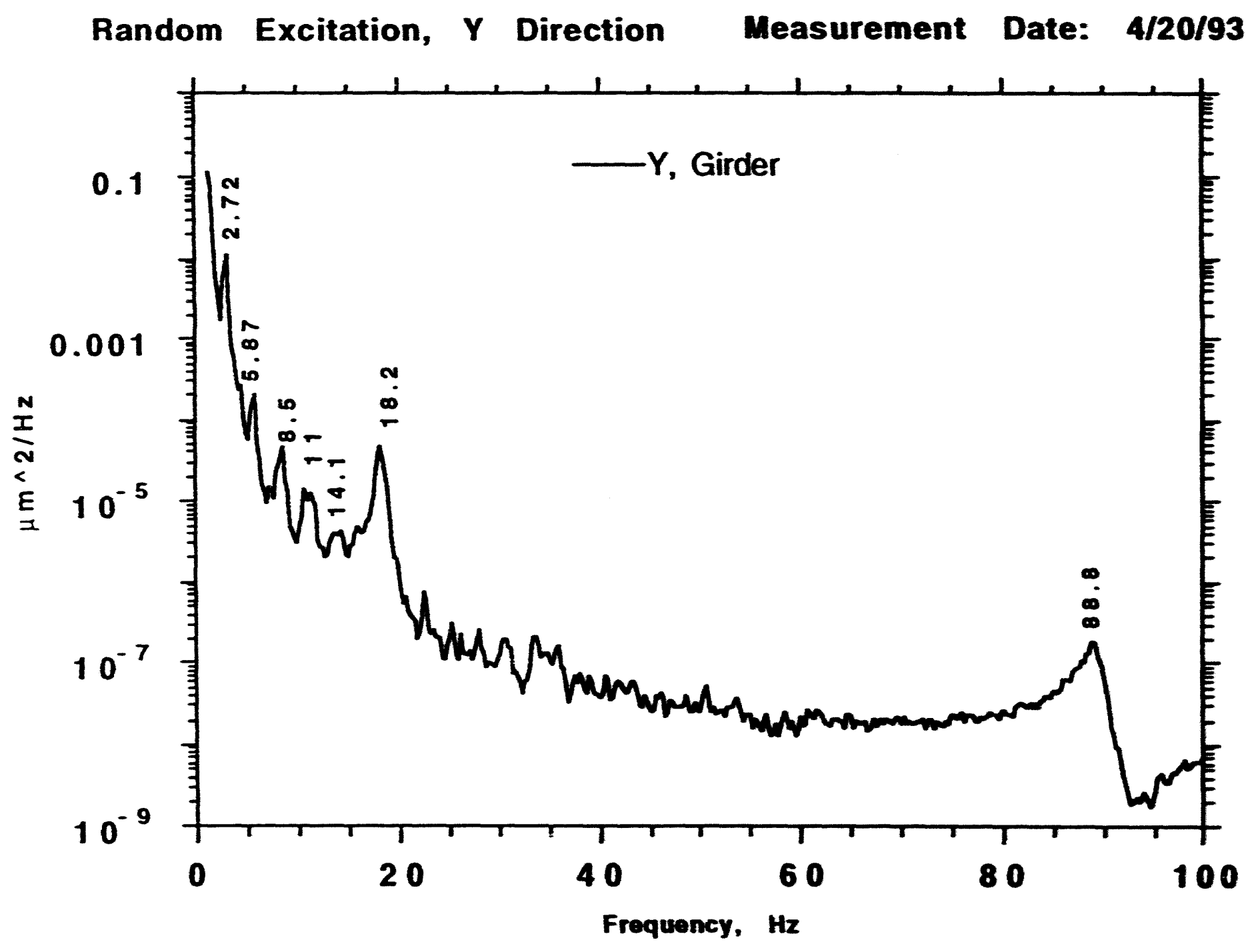


SYNCHROTRON GIRDER RESPONSE Girder \# B2C61

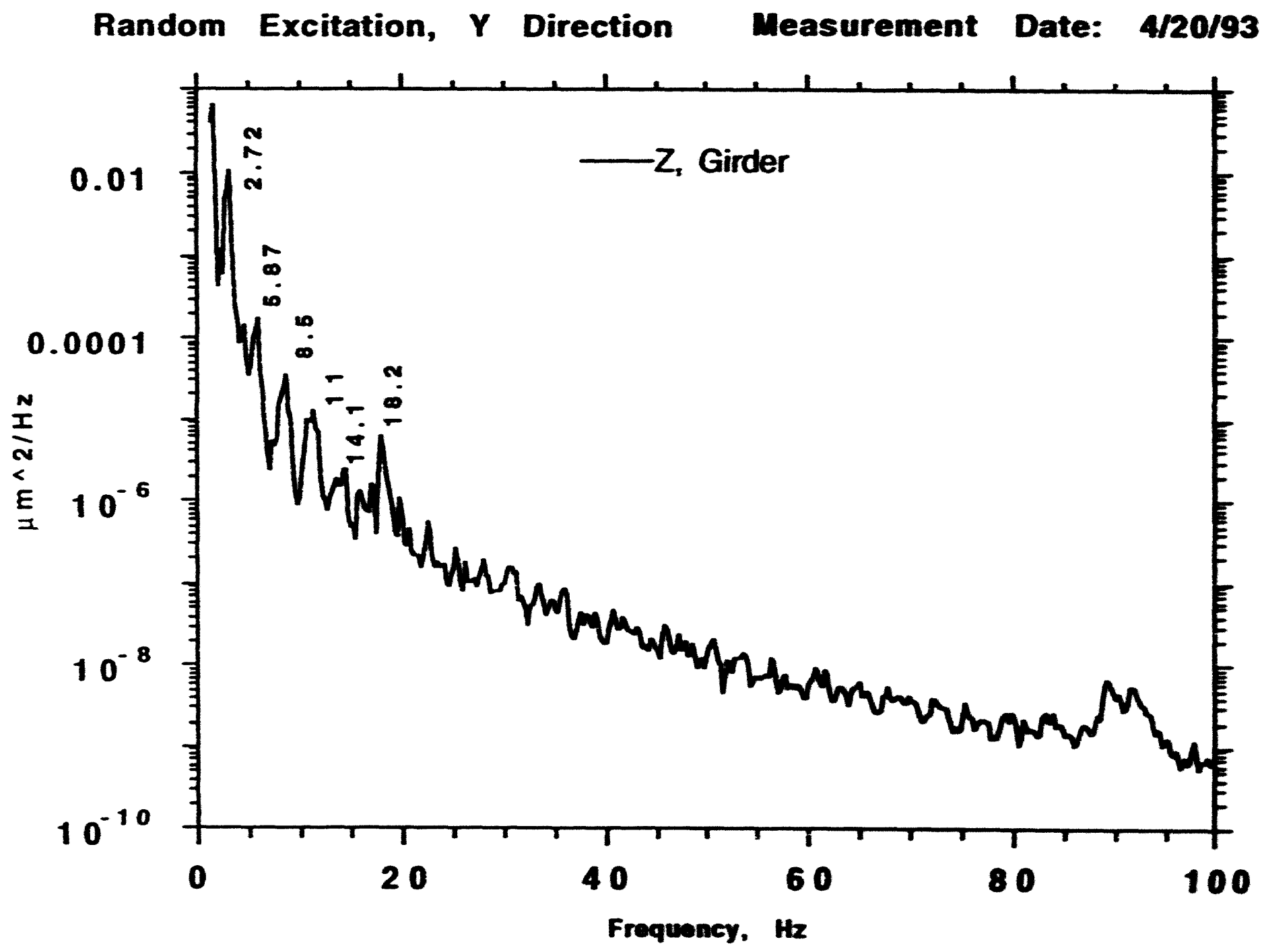




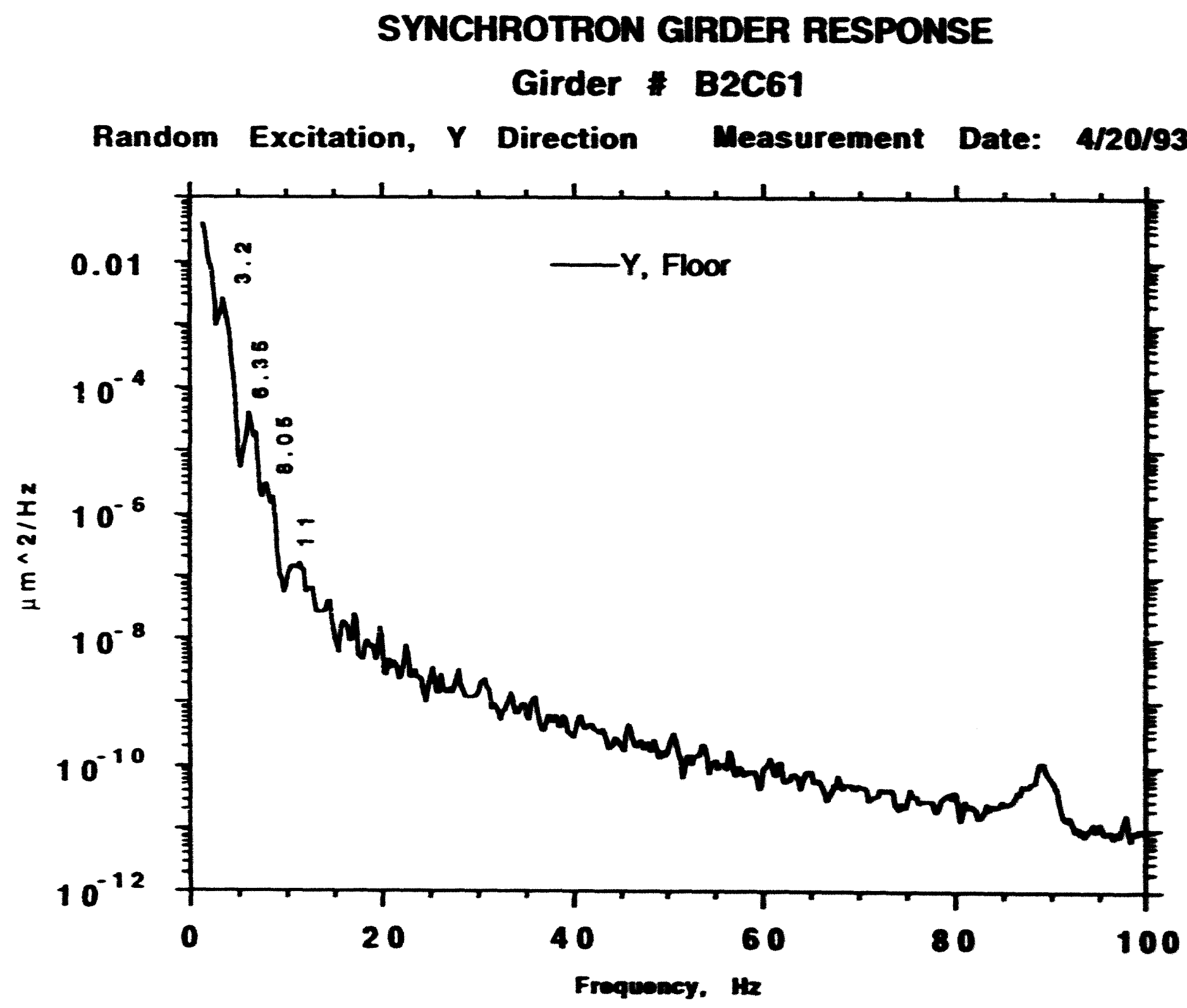


SYNCHROTRON GARDER RESPONSE

Girder B2C61

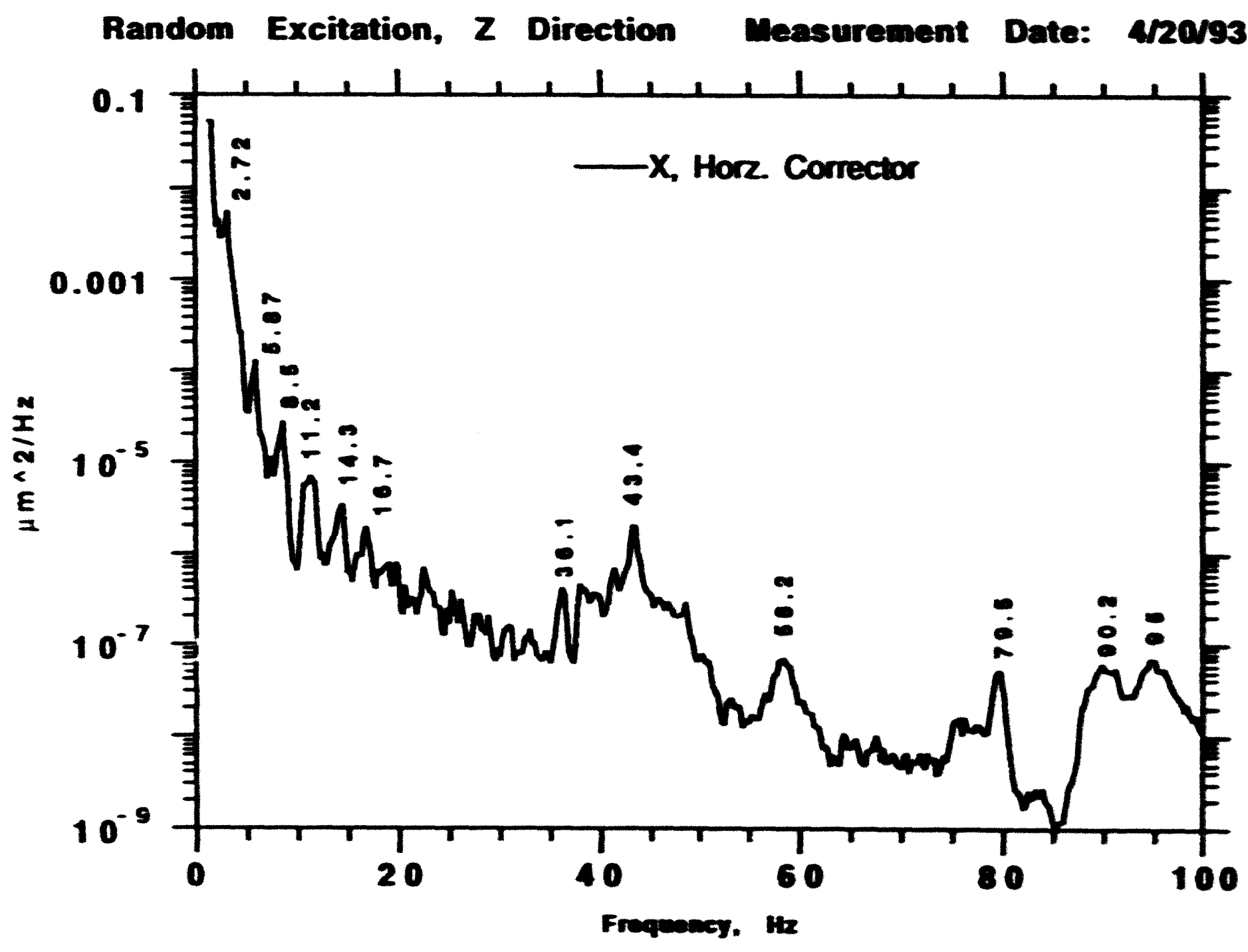




\section{SYNCHROTRON GIRDER RESPONSE}

Girder B2C61

Random Excitation, $Z$ Direction Measurement Date: 4/20/93

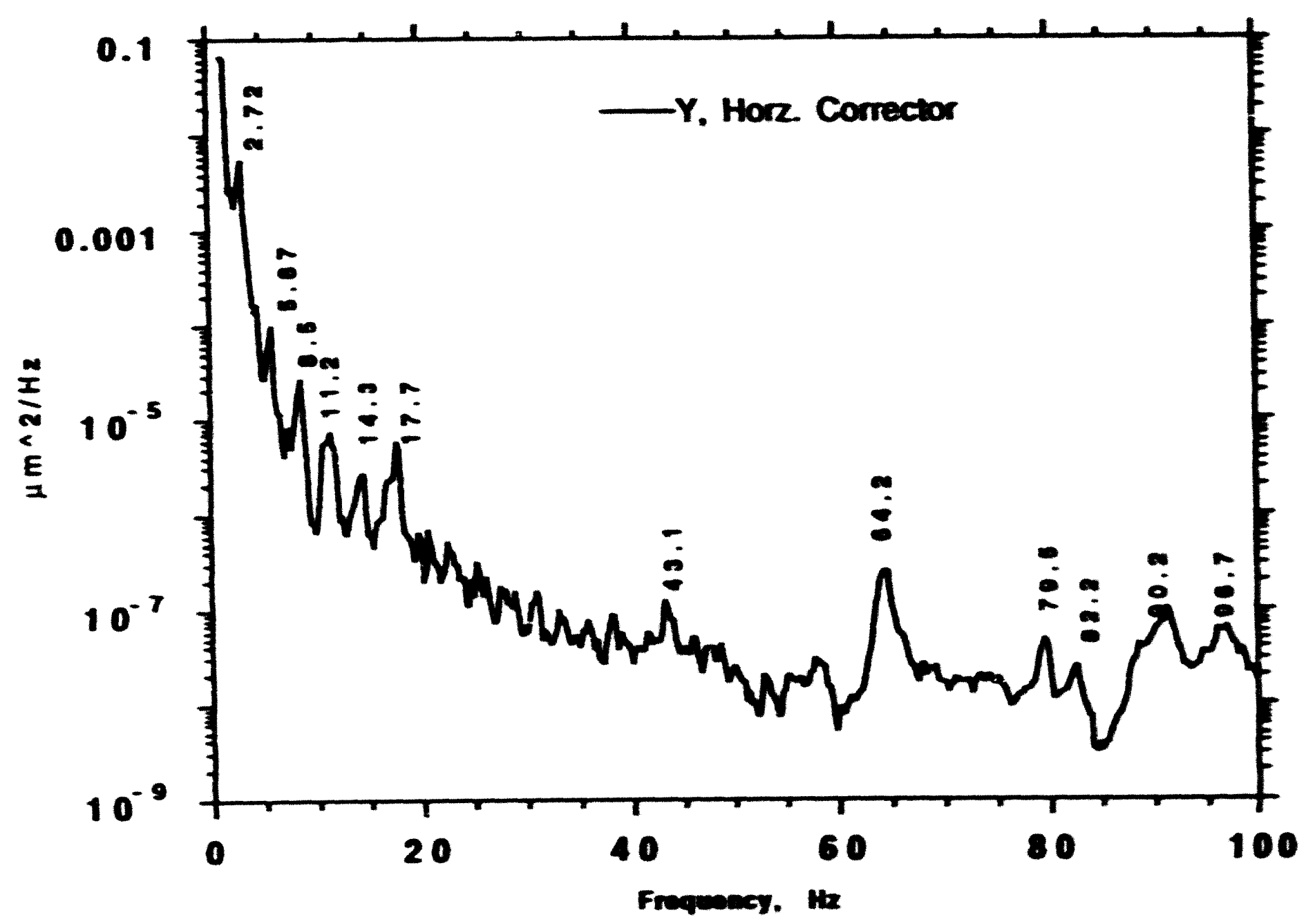


SYNCHROTRON GIRDER RESPONSE

Girder B2C61

Random Excitation, Z Direction Measurement Date: $1 / 20 / 93$

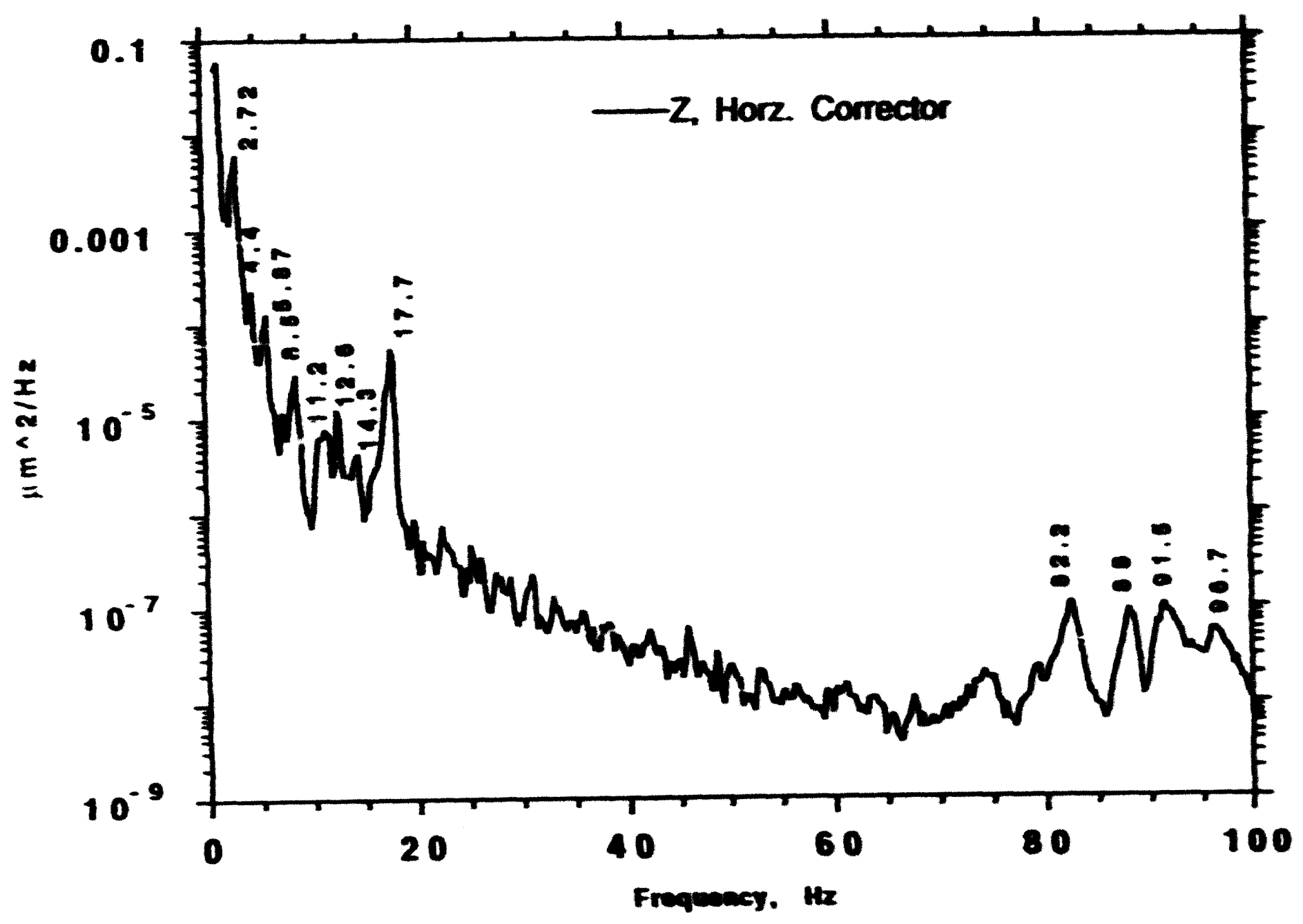




\section{SYNCHROTRON GIRDER RESPONSE}

\section{Girder B2C61}

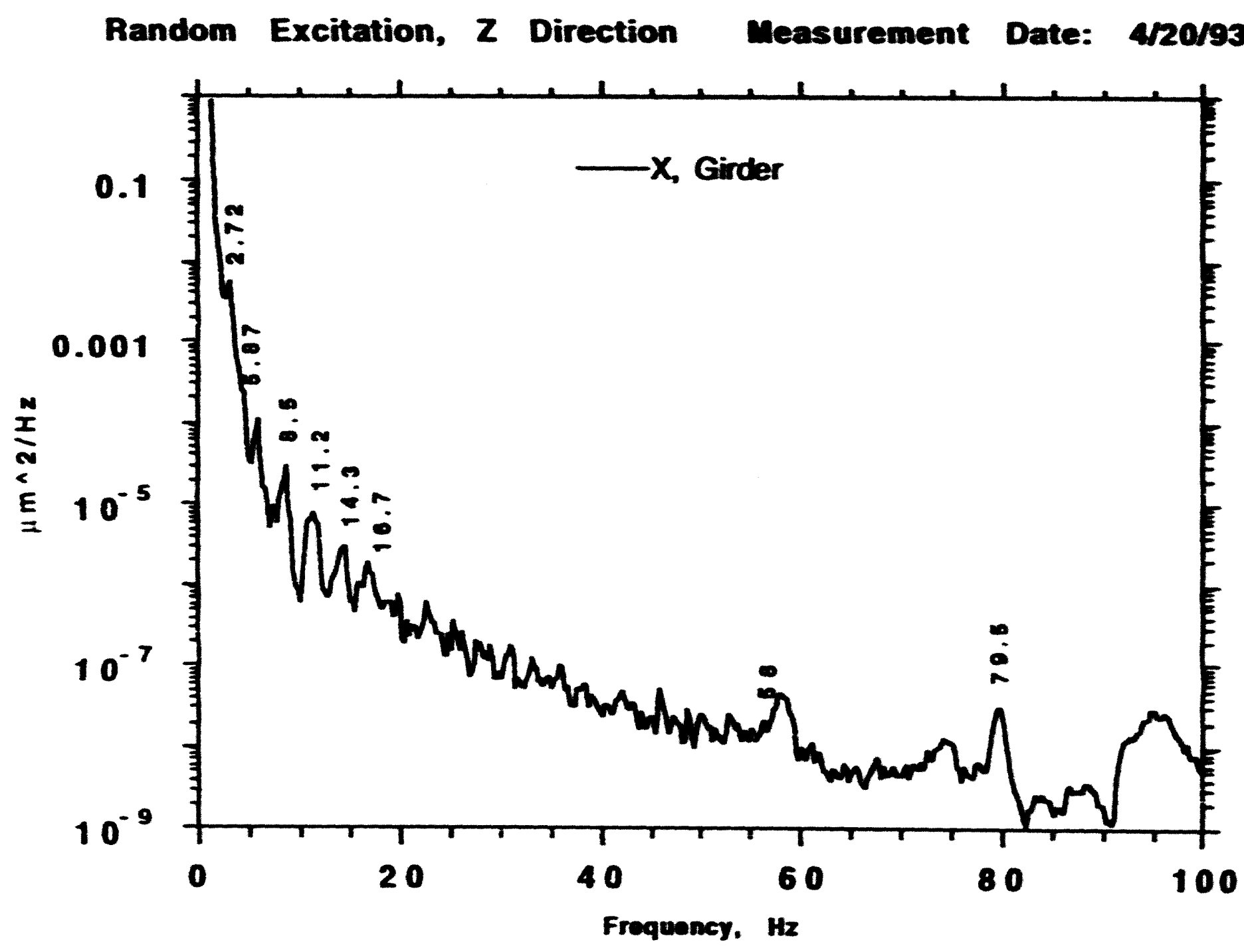




\section{SYNCHROTRON GIRDER RESPONSE}

Girder \# B2C61

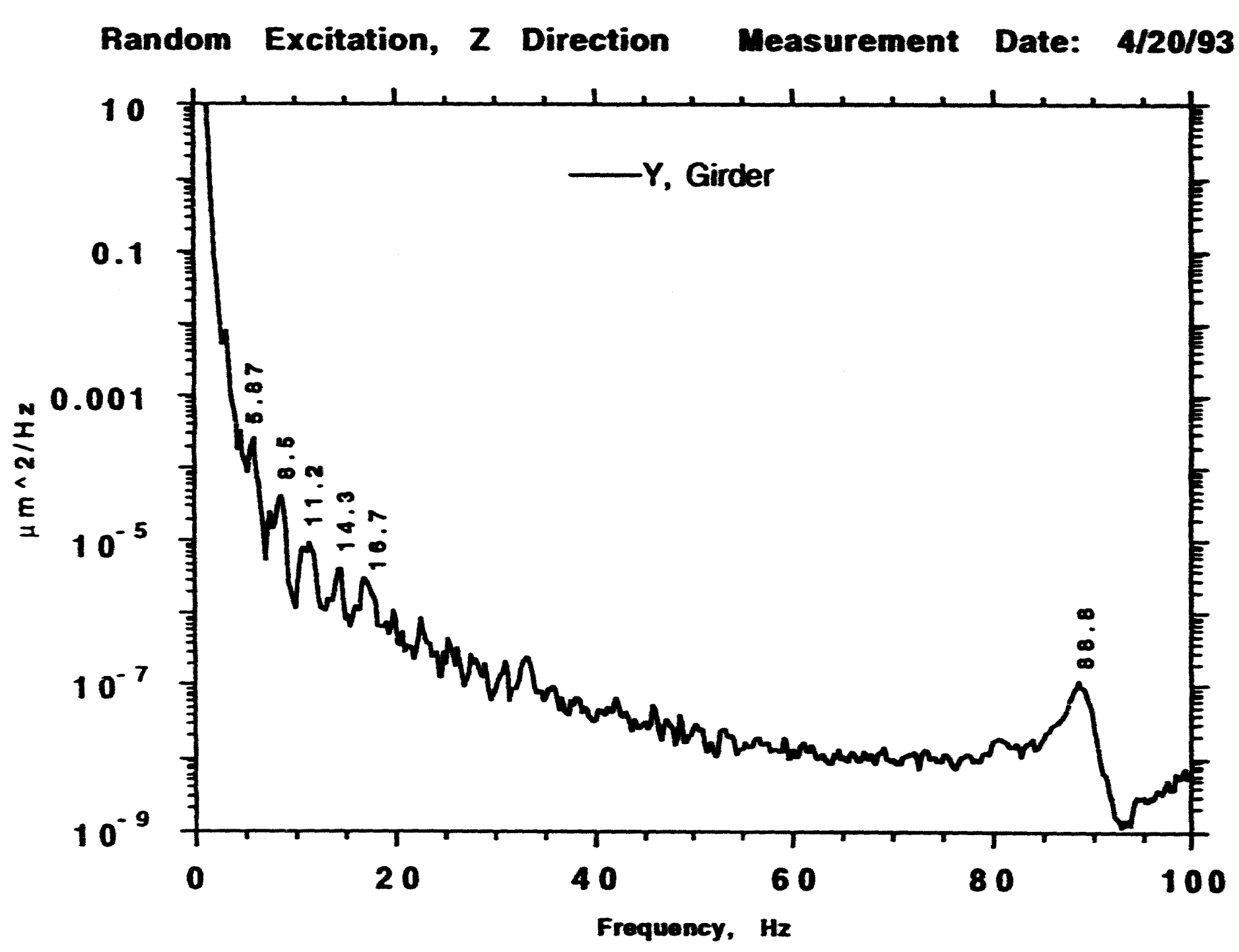




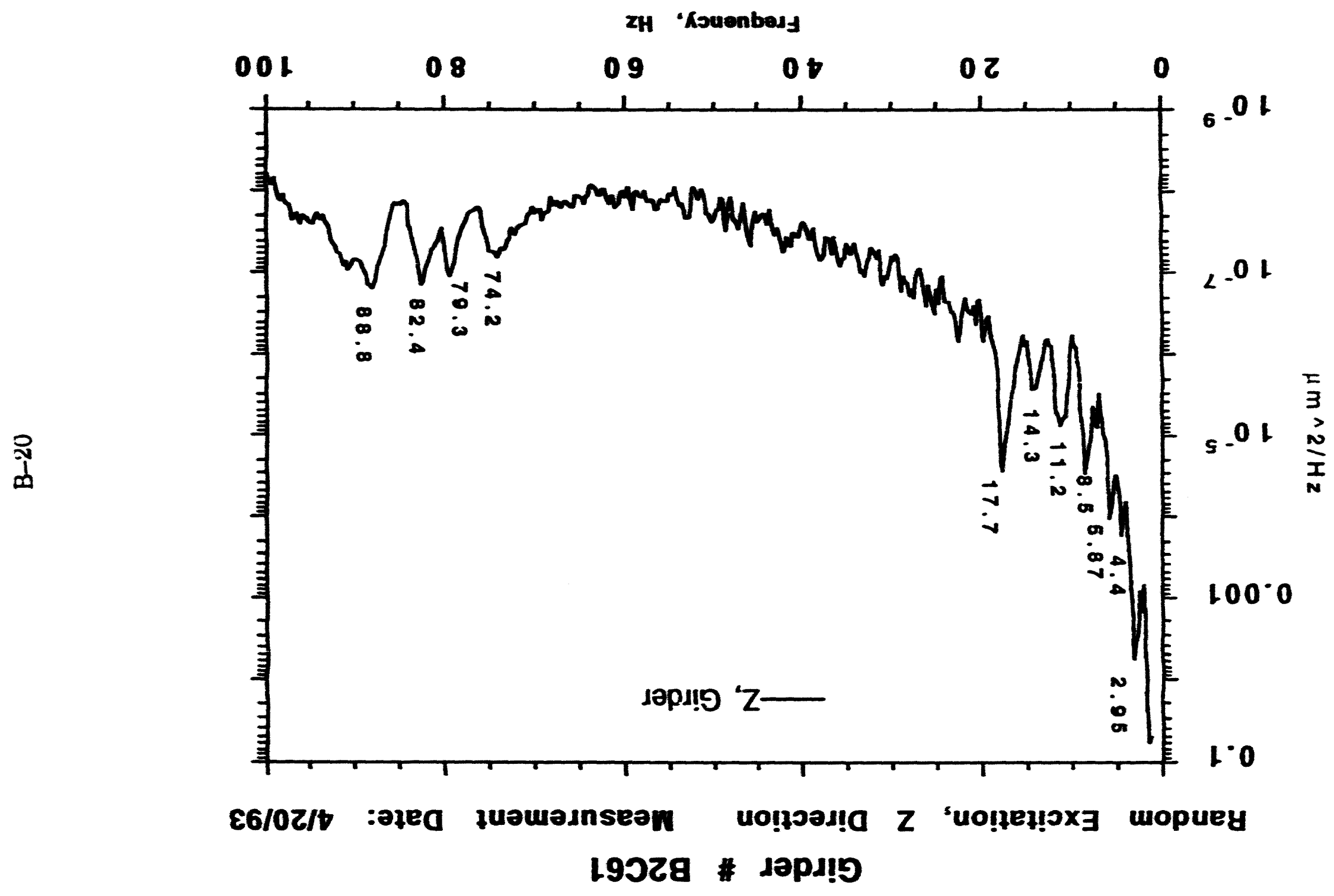

ISNOdS 


\section{SYNCHROTRON GIRDER RESPONSE}

Girder \# B2C61

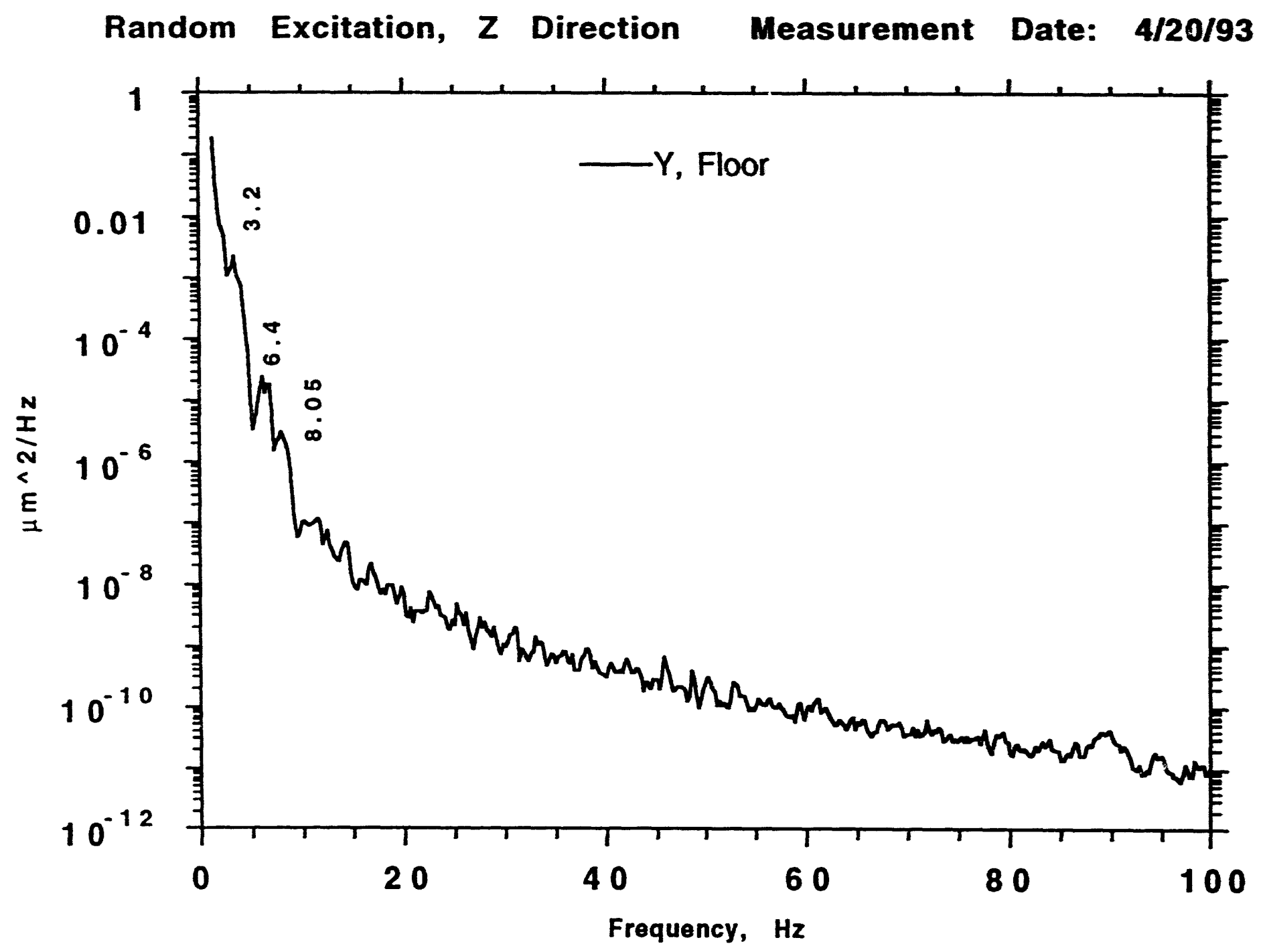




\section{SYNCHROTRON GIRDER RESPONSE}

Girder \# B2C61

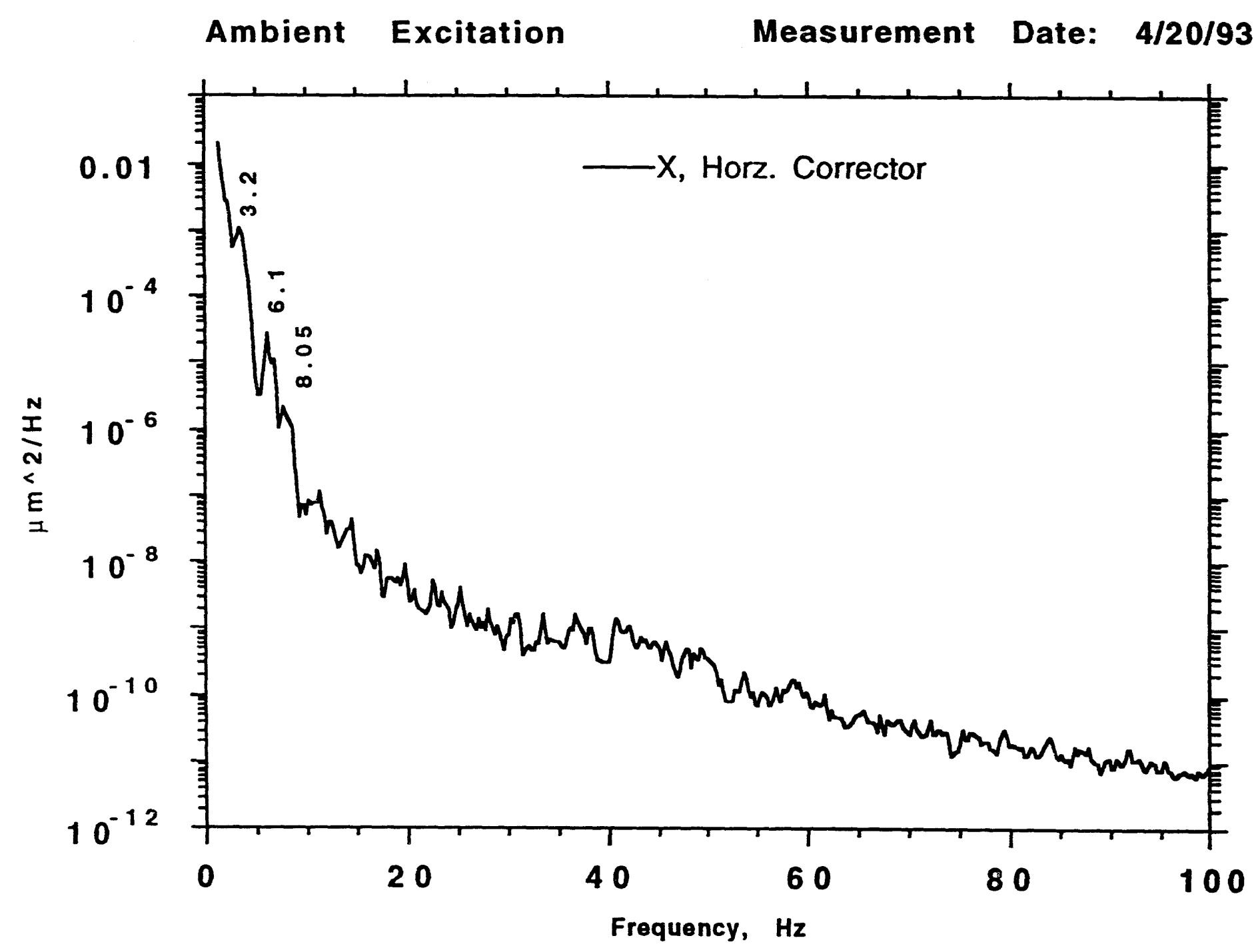




\section{SYNCHROTRON GIRDER RESPONSE}

Girder \# B2C61

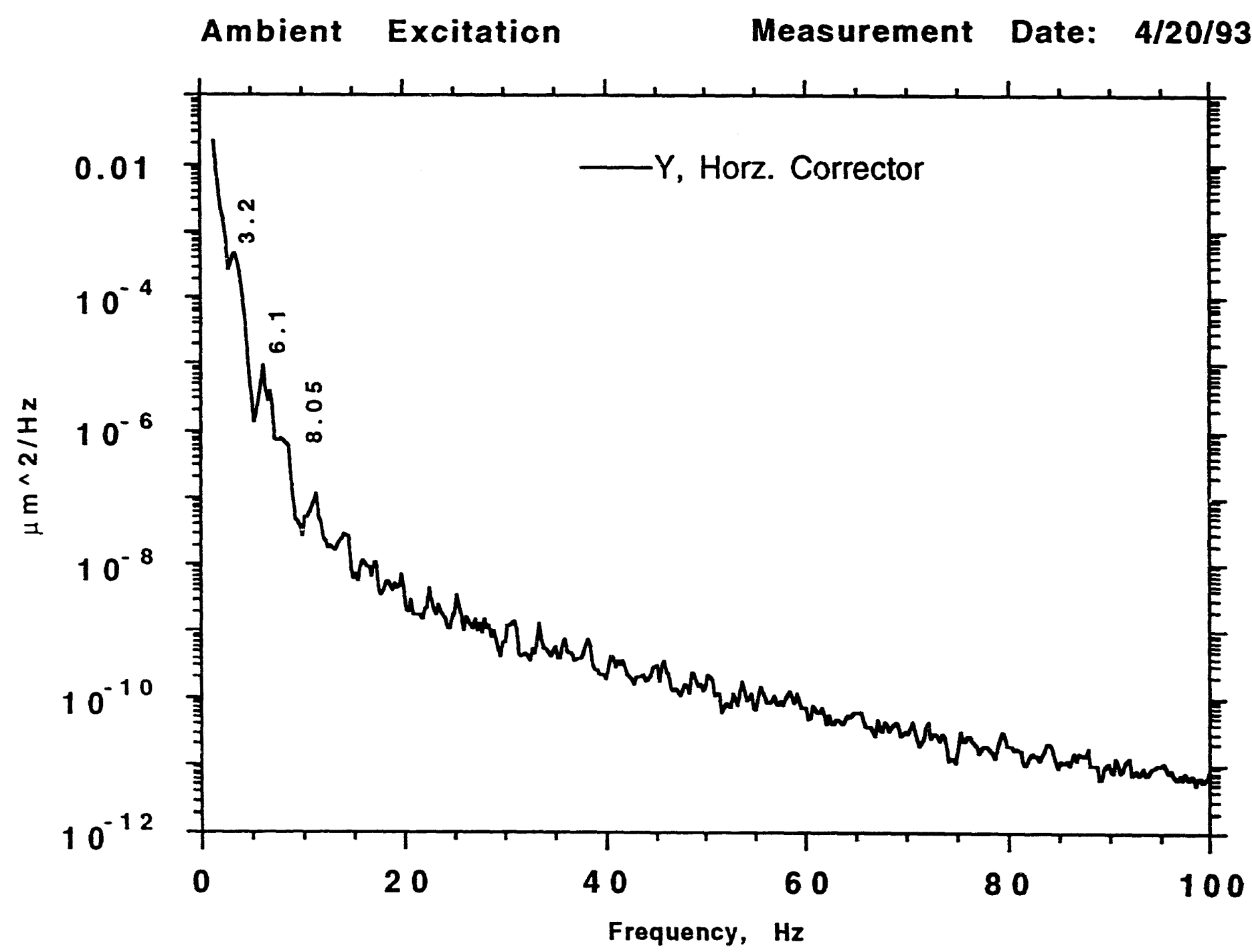




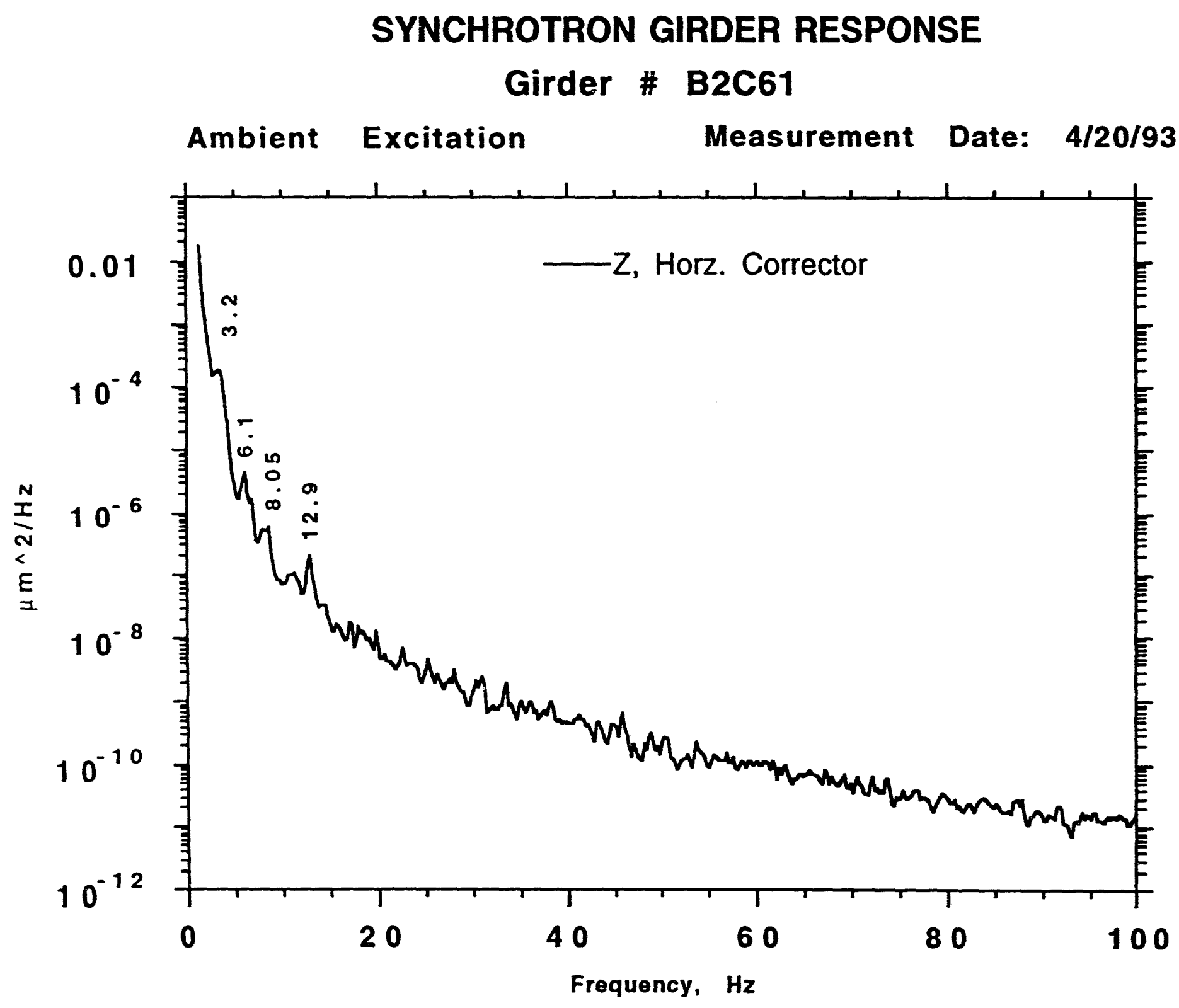




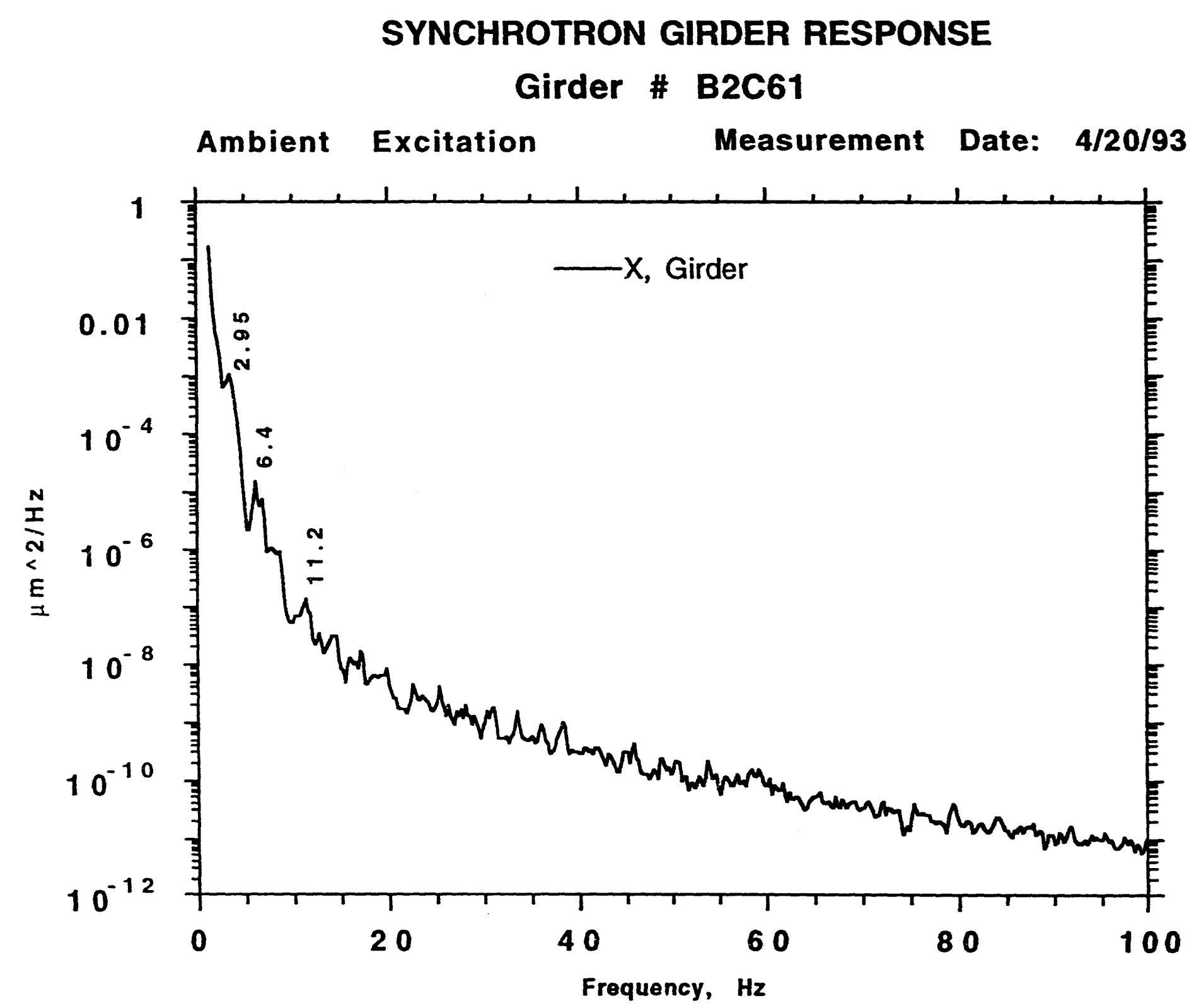




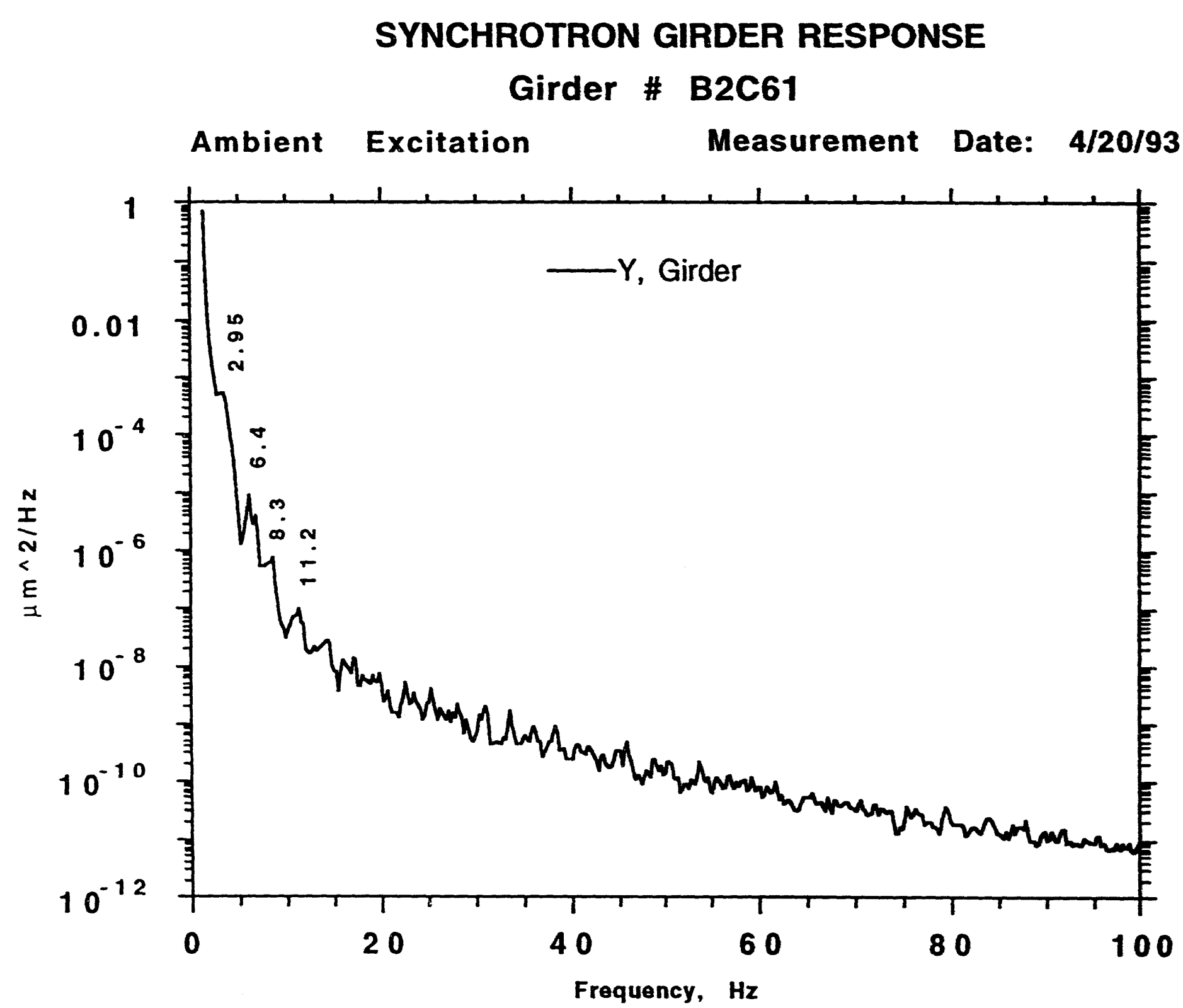




\section{SYNCHROTRON GIRDER RESPONSE}

\section{Girder \# B2C61}

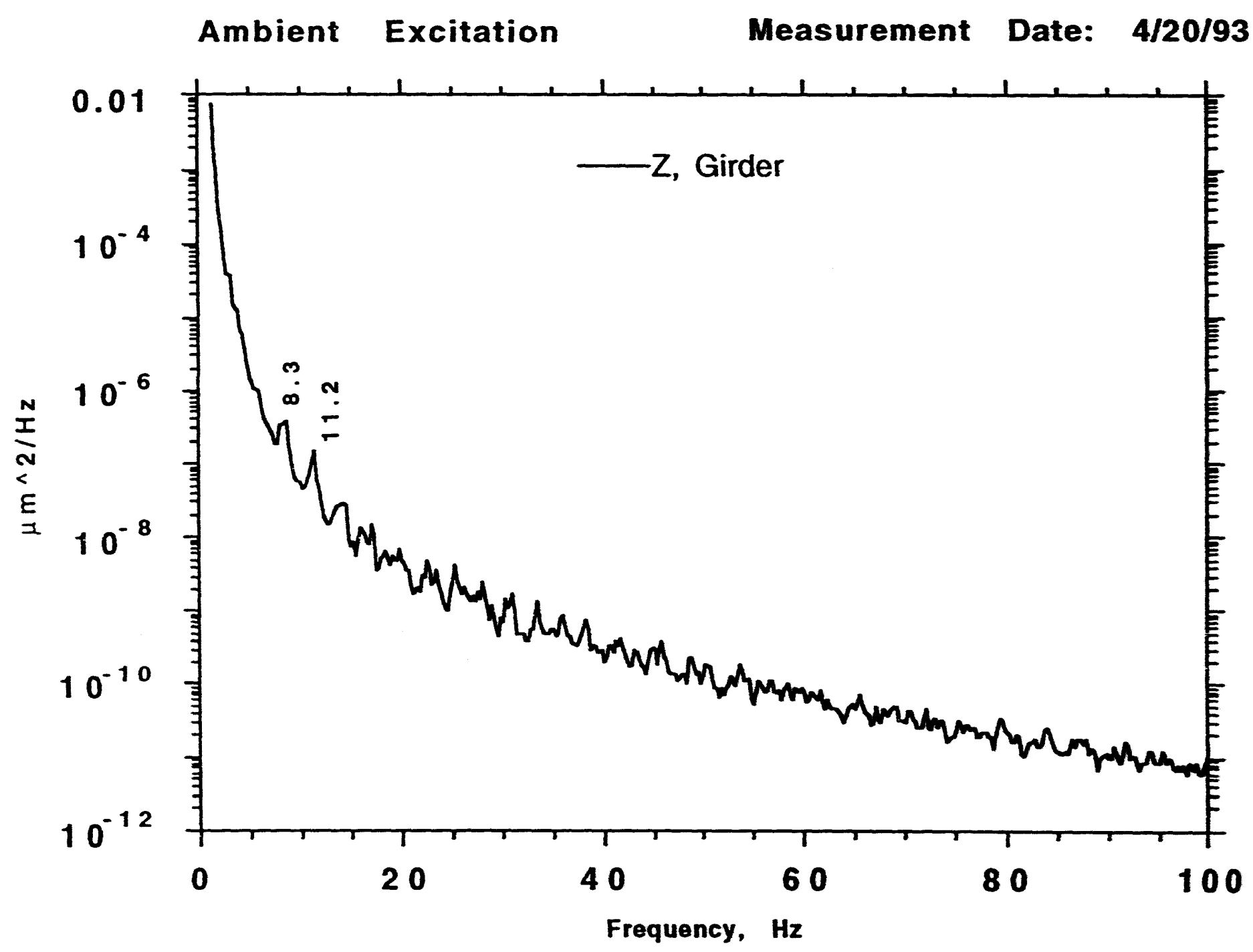




\section{SYNCHROTRON GIRDER RESPONSE}

Girder \# B2C61

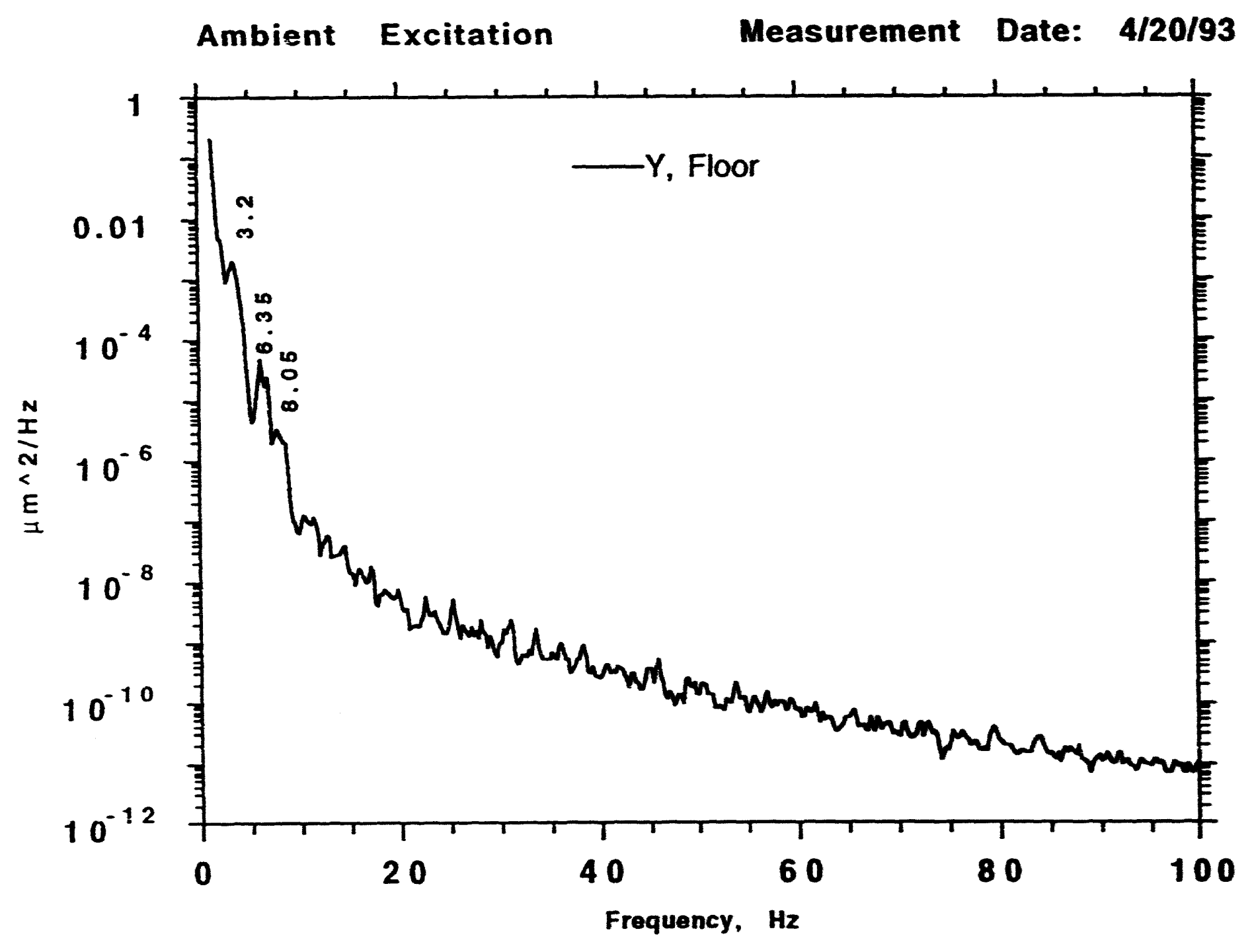


Appendix C: Quadrupole magnet response 


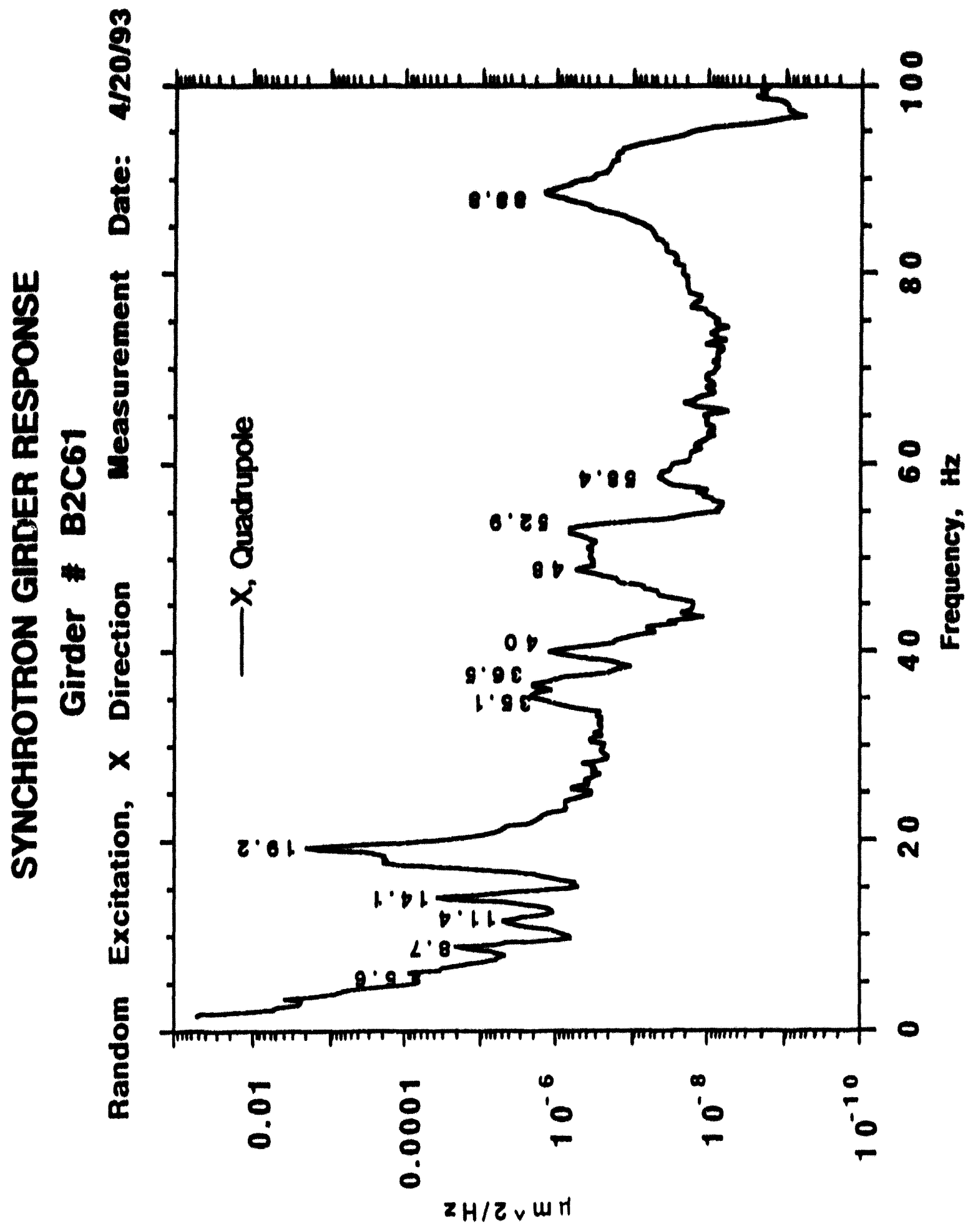




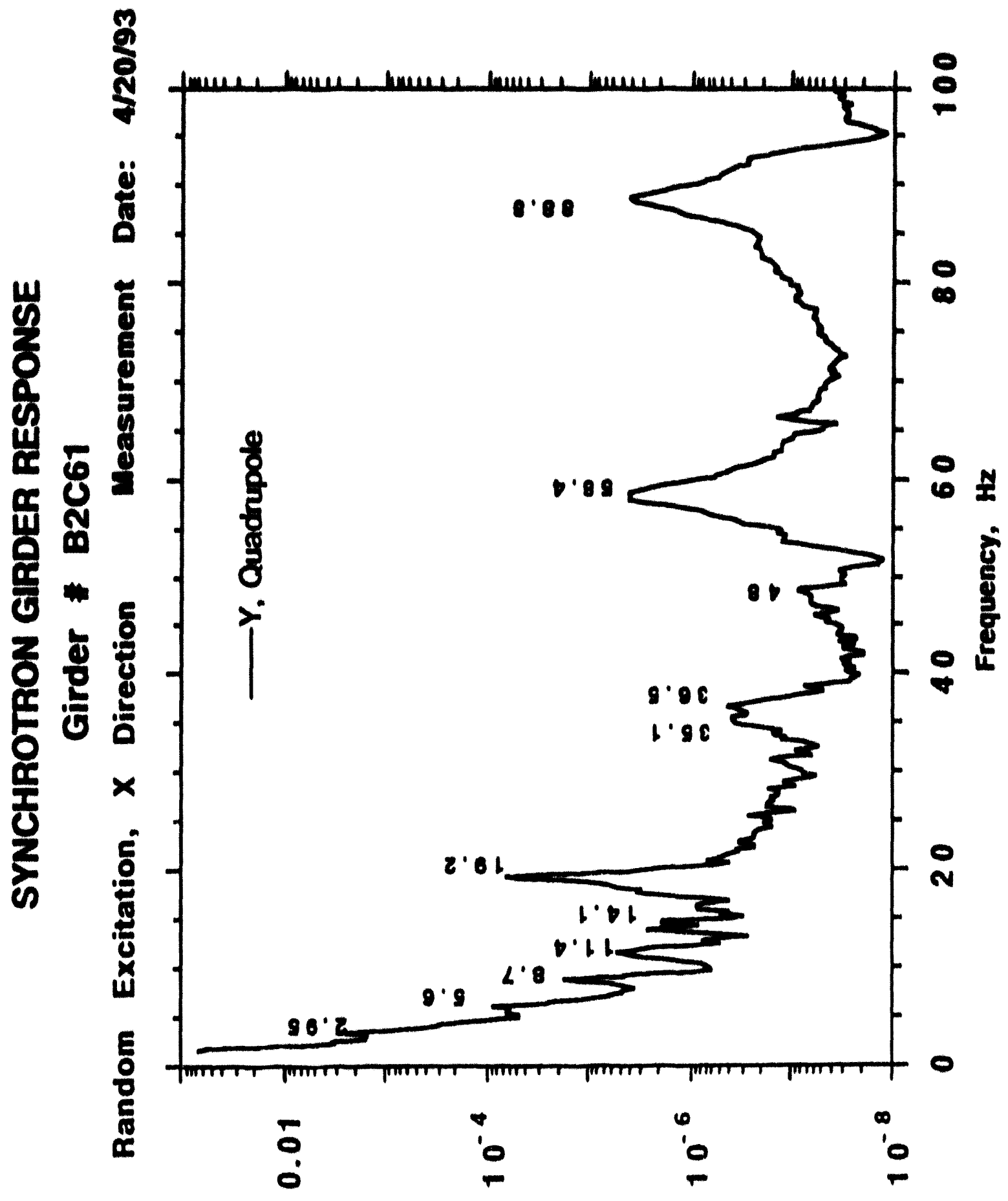

$2 H / 2 \vee W H$ 


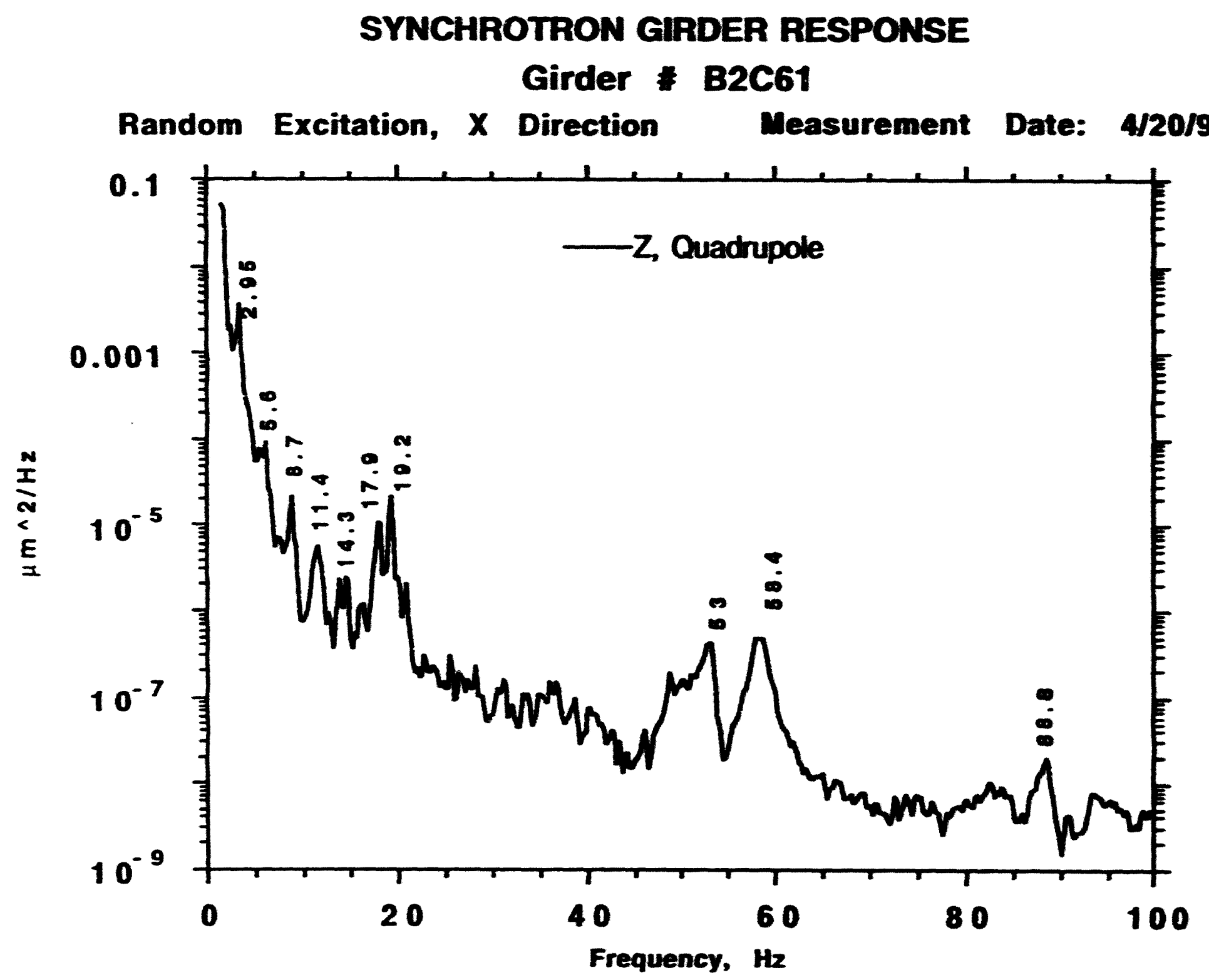

$\stackrel{\rho}{\infty}$ 


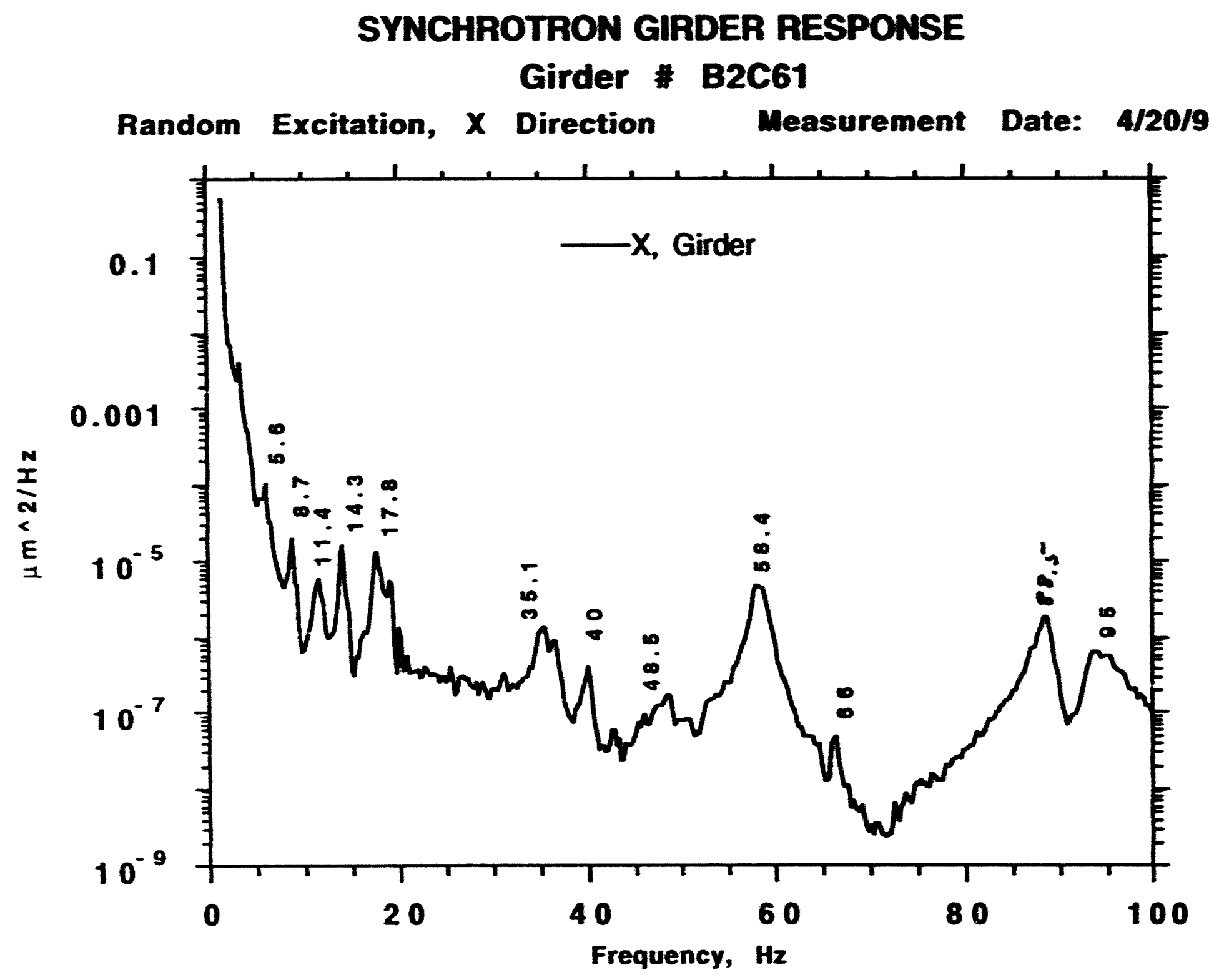




\section{SYNCHROTRON GIRDER RESPONSE}

\section{Girder \# B2C61}

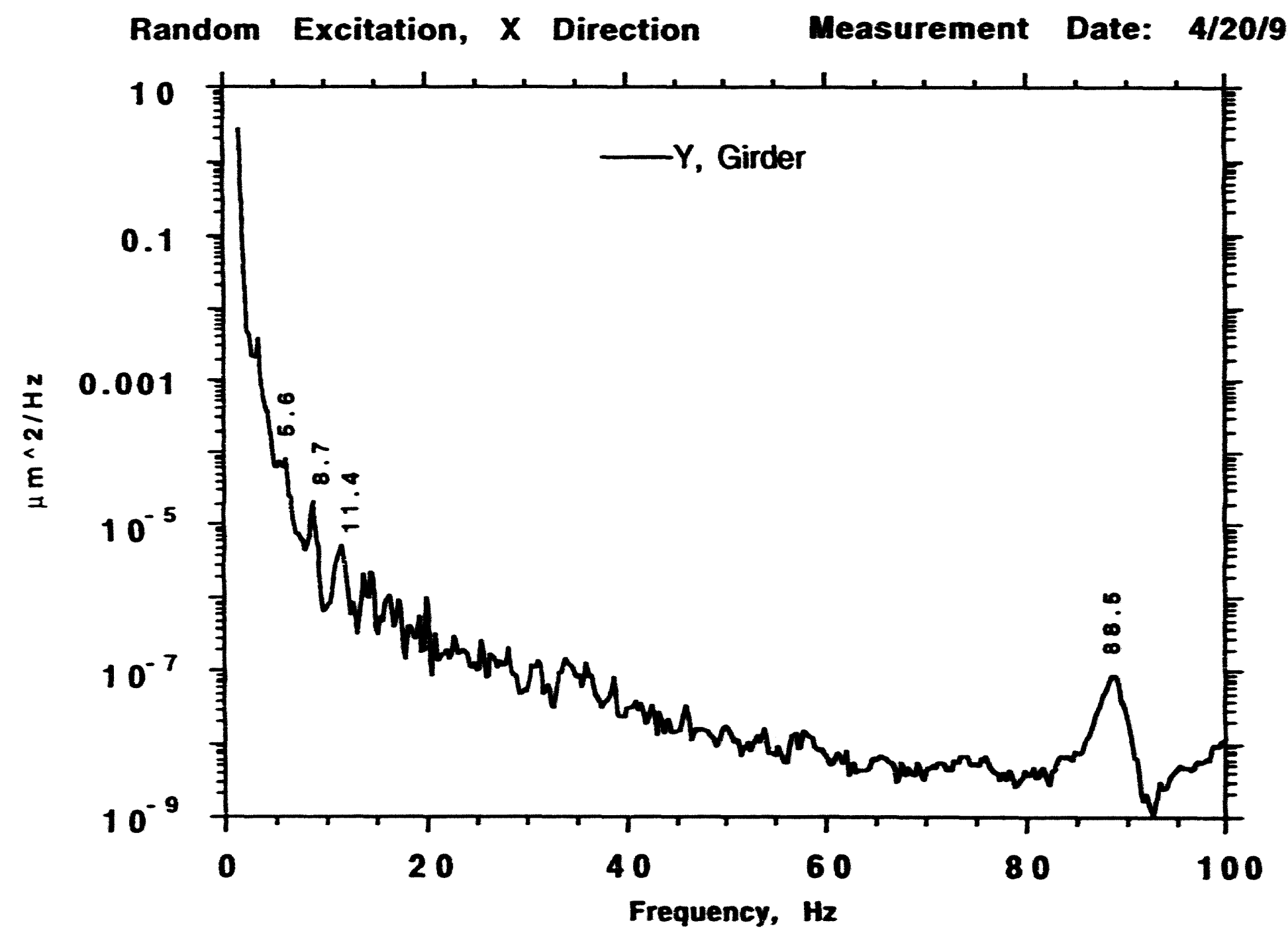




\section{SYNCHROTRON GIRDER RESPONSE}

Girder \# B2C61

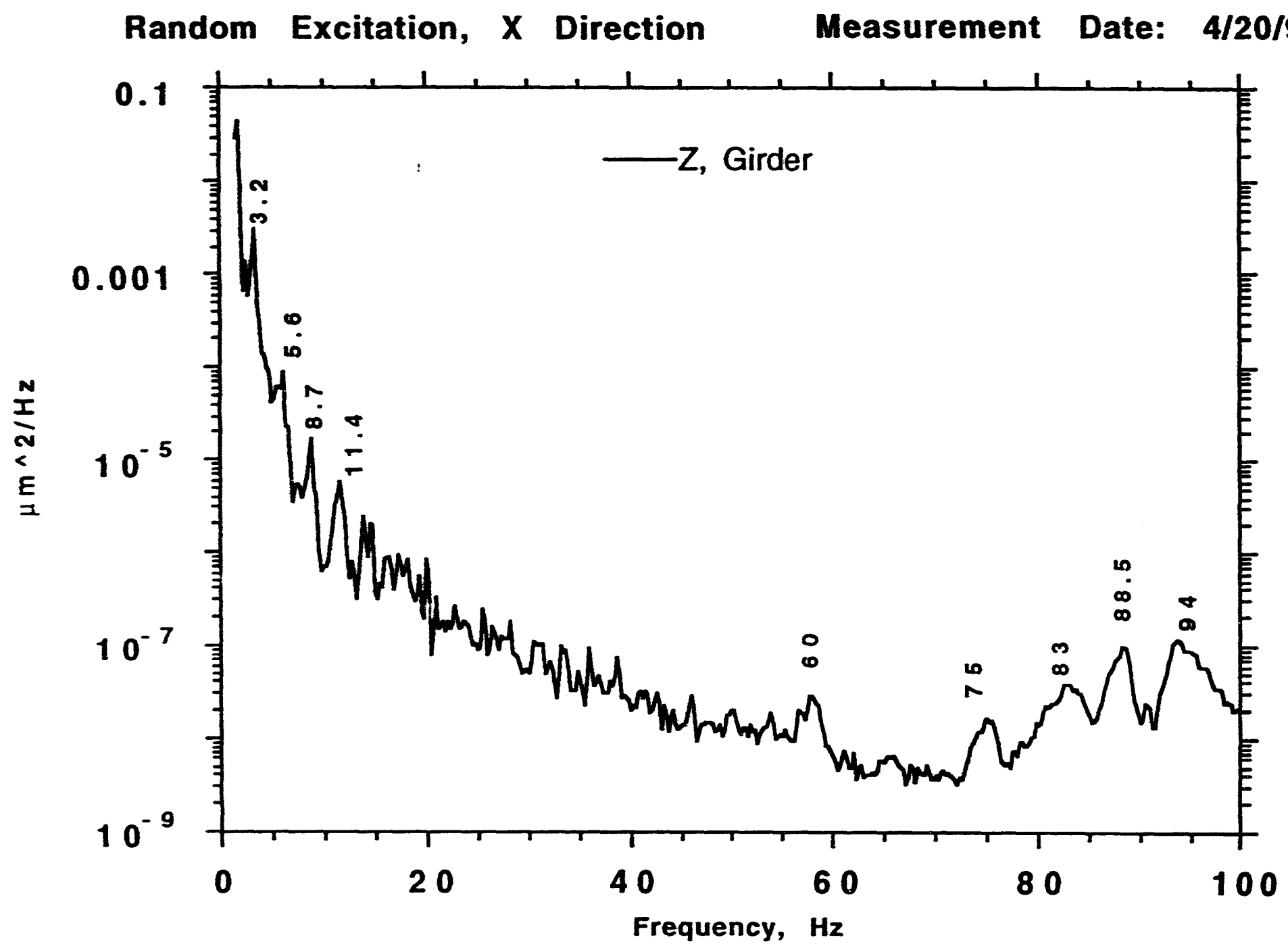




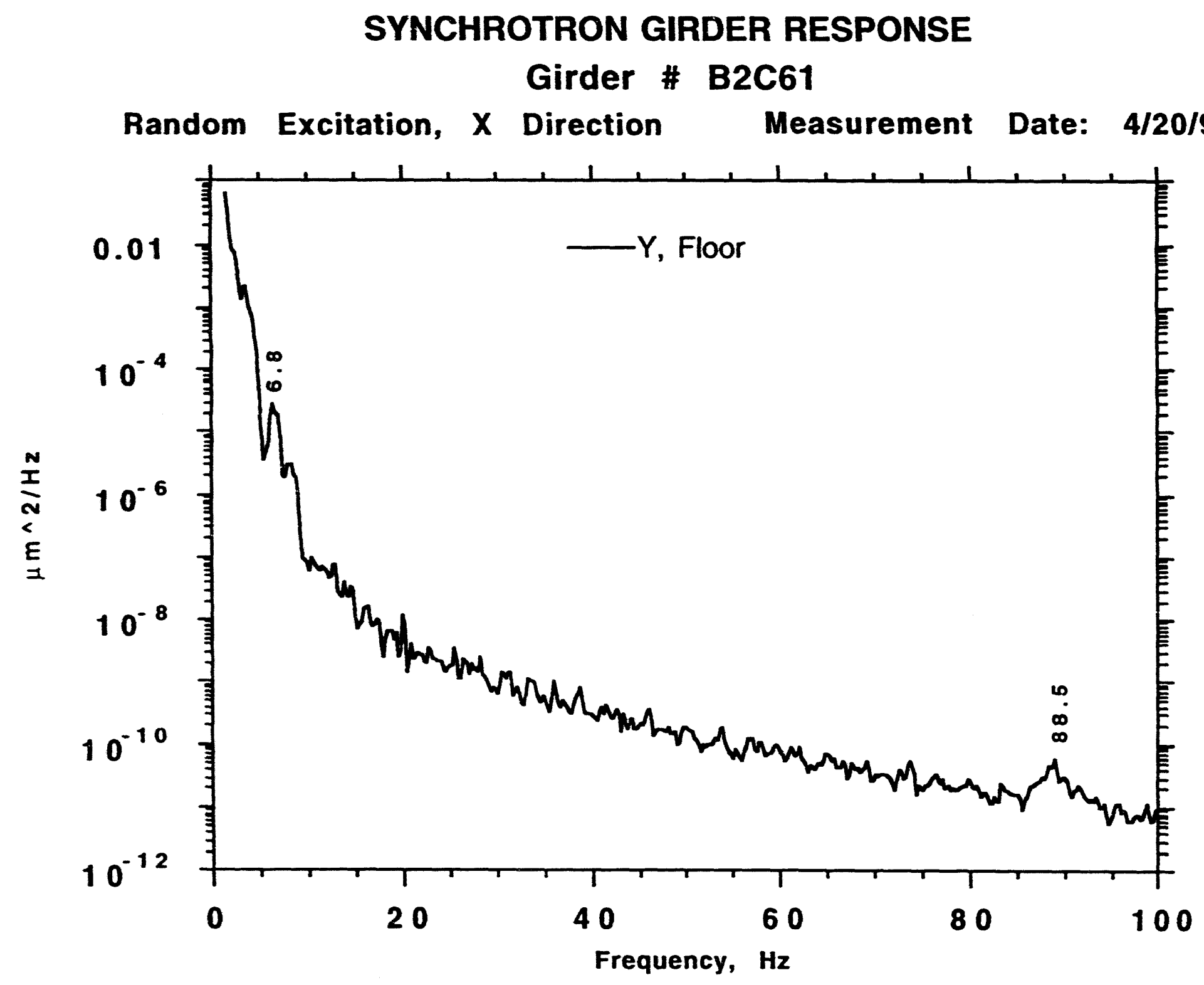




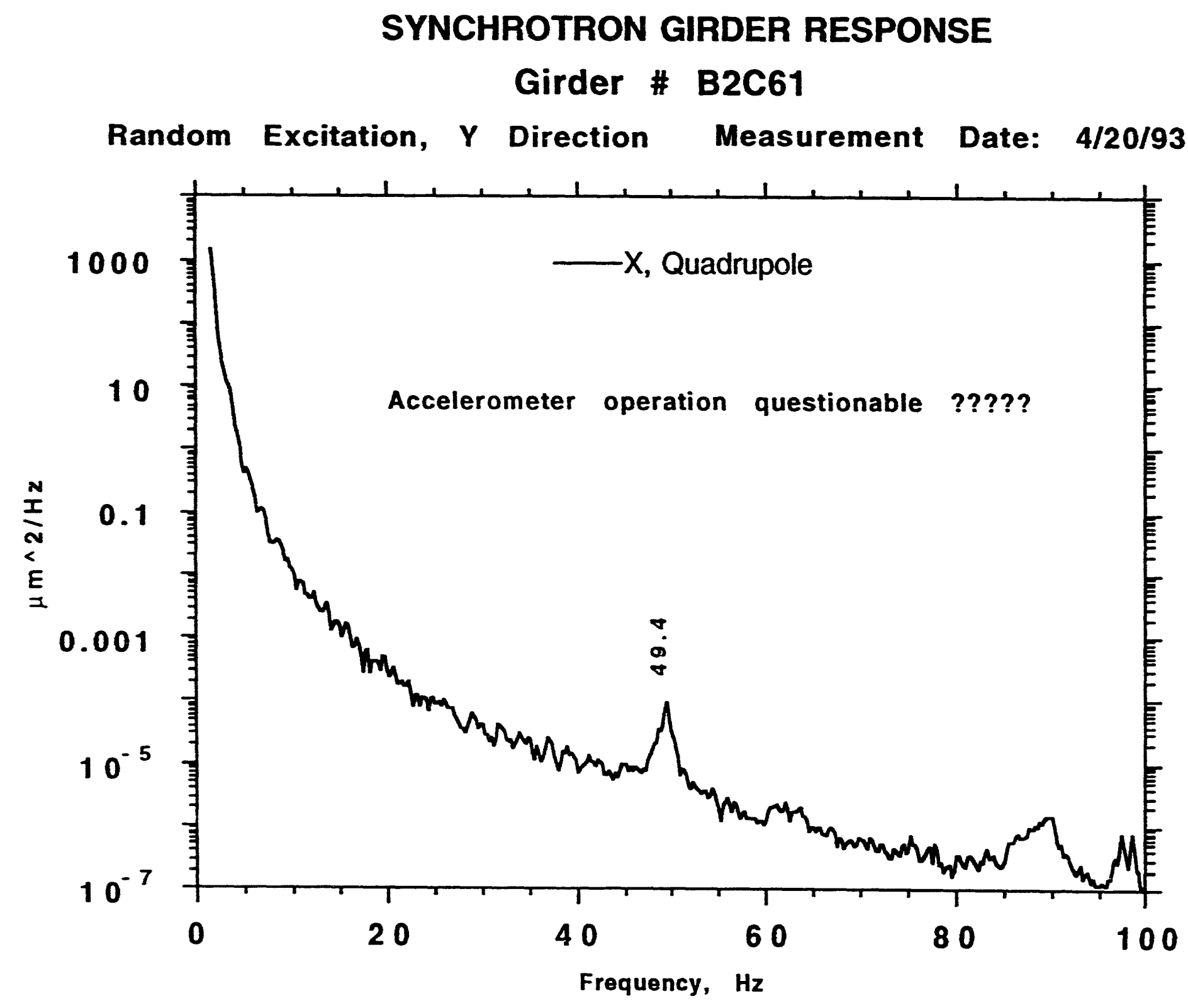




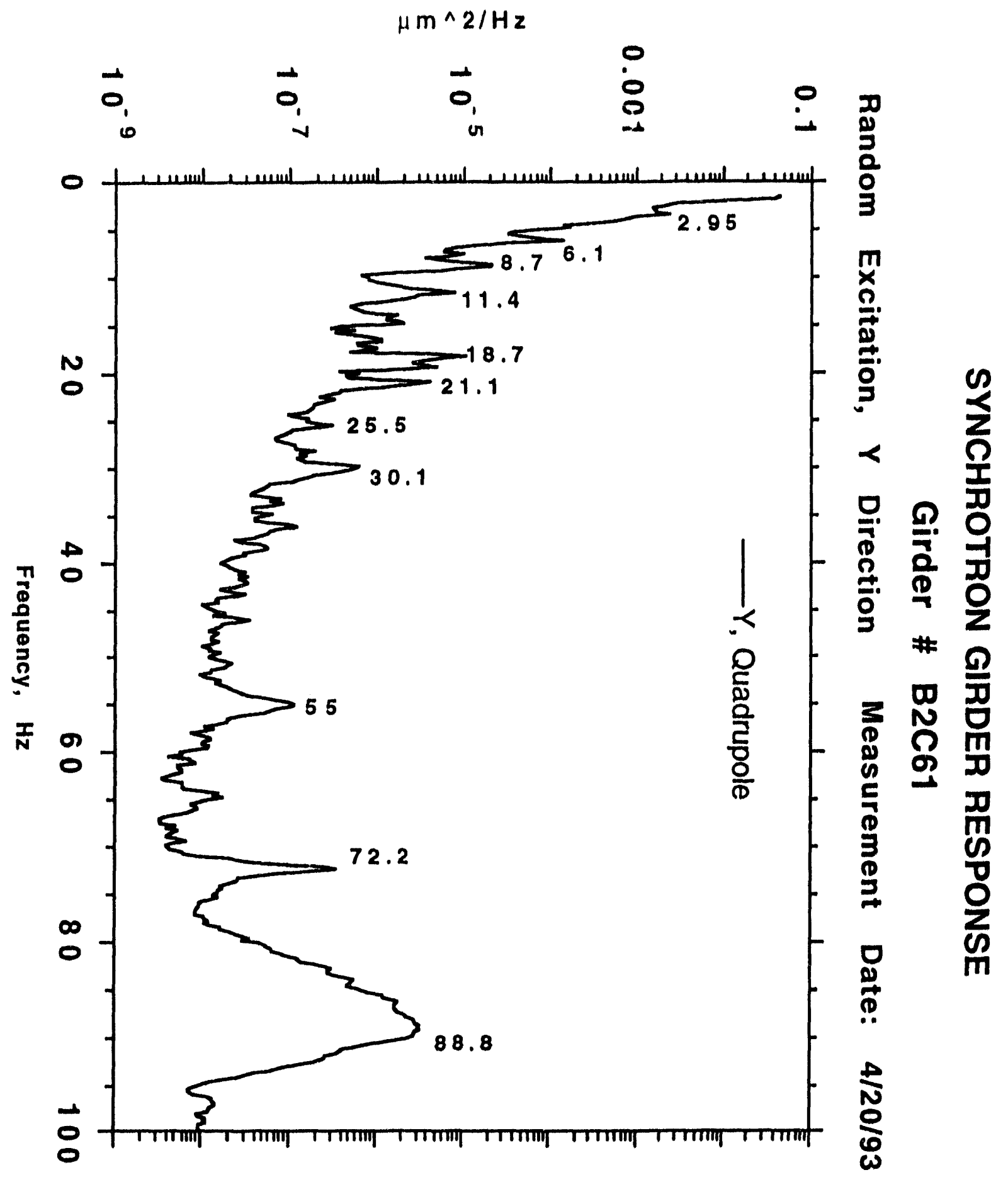




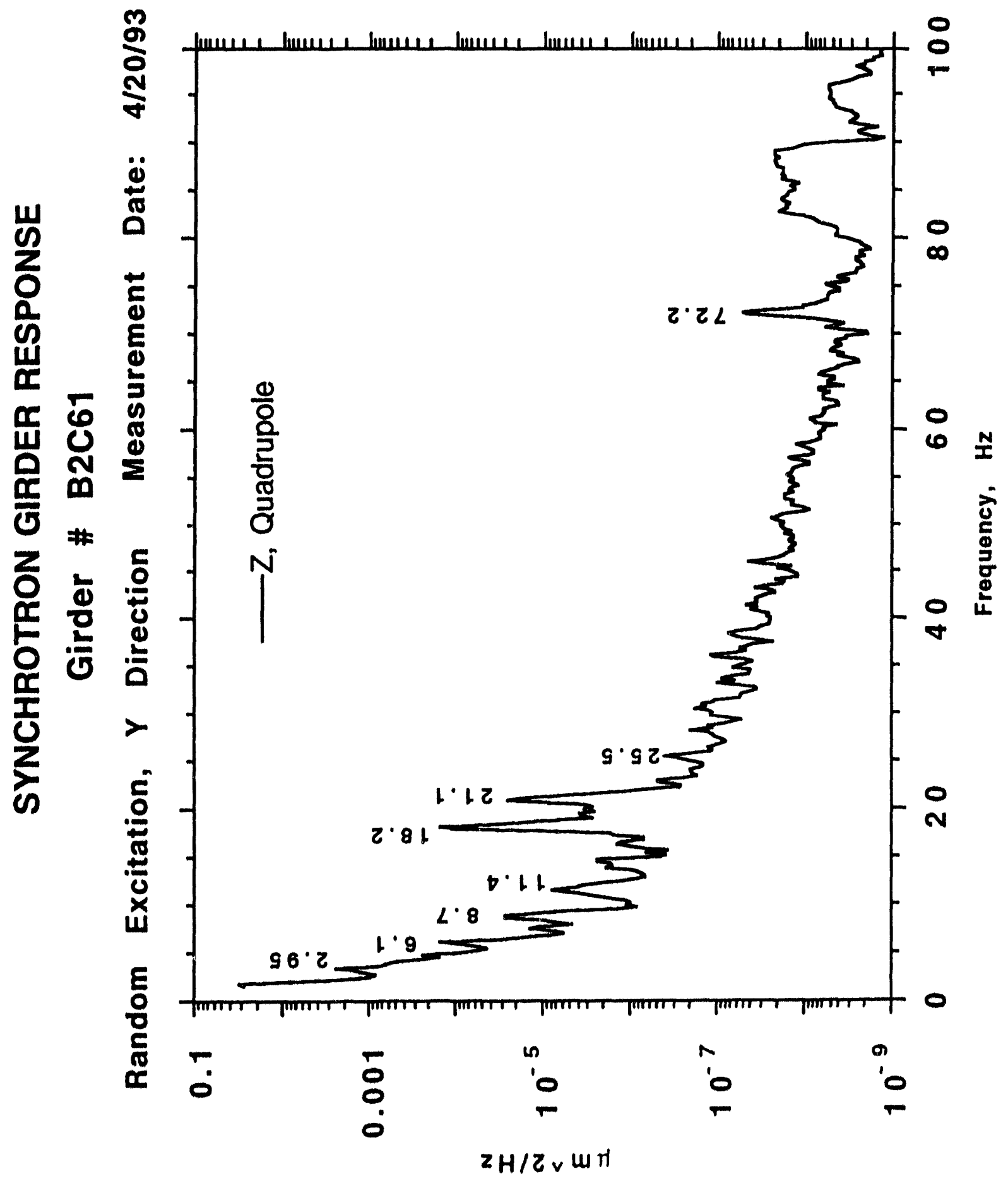




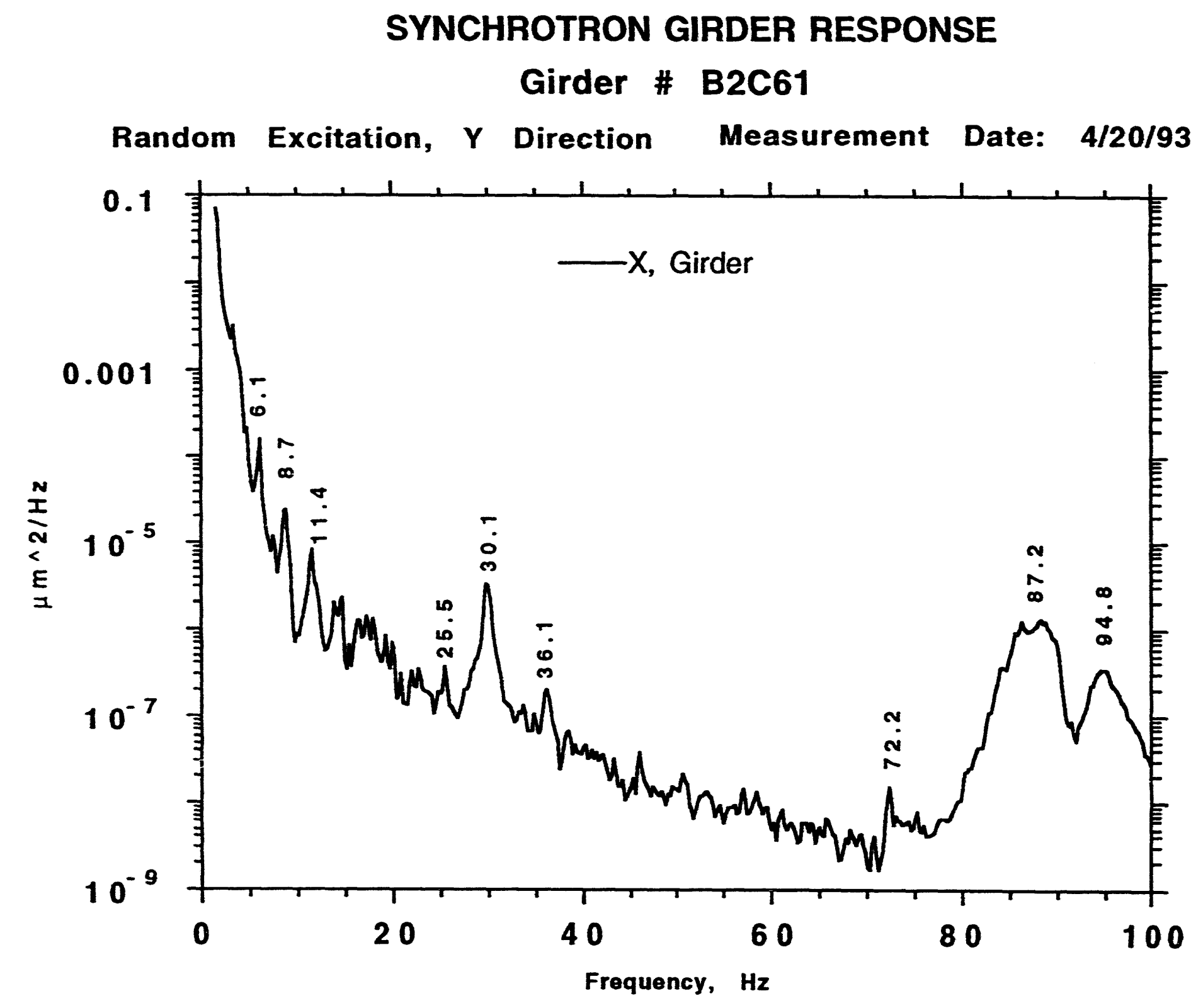




\section{SYNCHROTRON GIRDER RESPONSE}

\section{Girder \# B2C61}

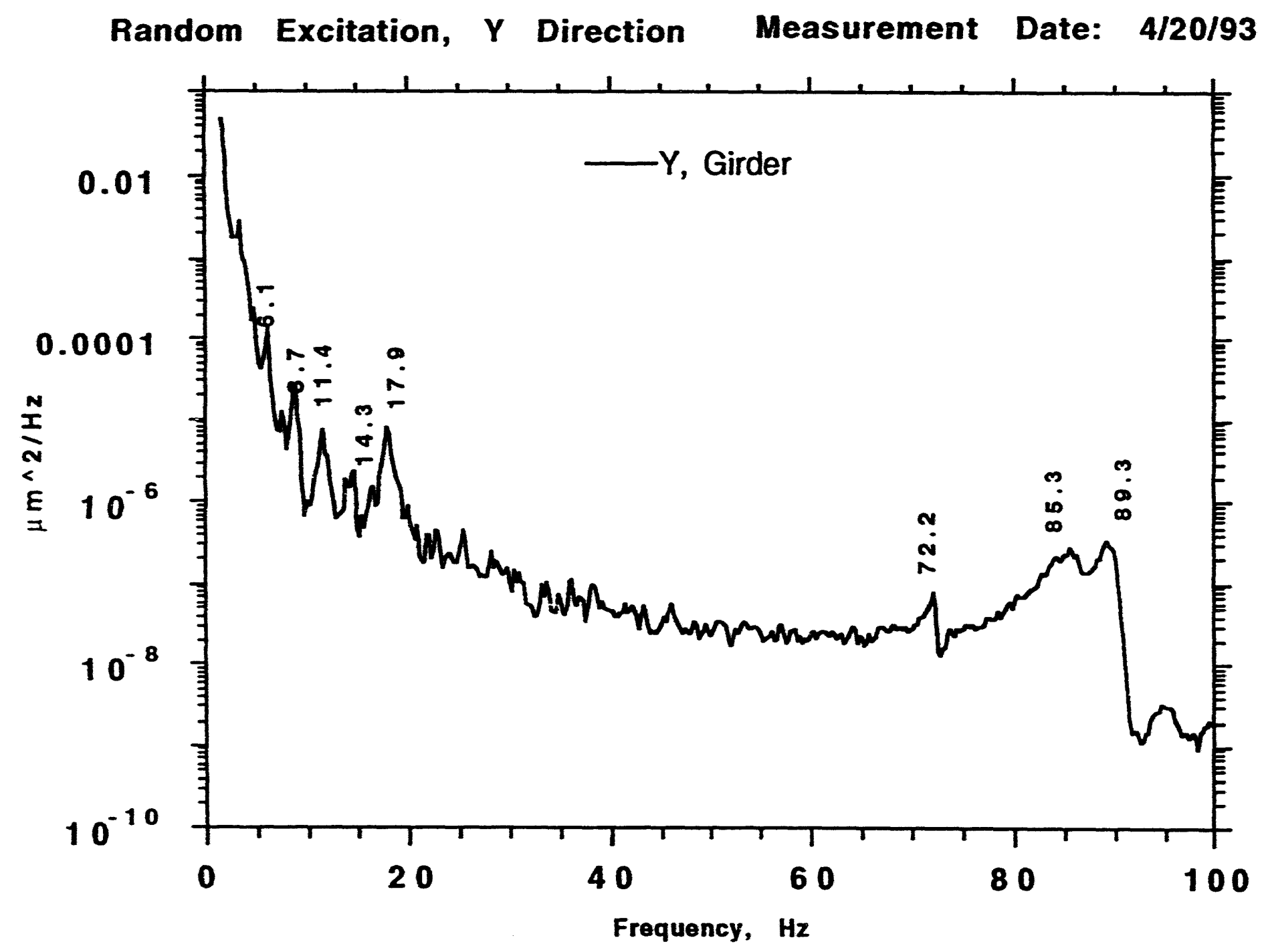




\section{SYNCHROTRON GIRDER RESPONSE}

\section{Girder \# B2C61}

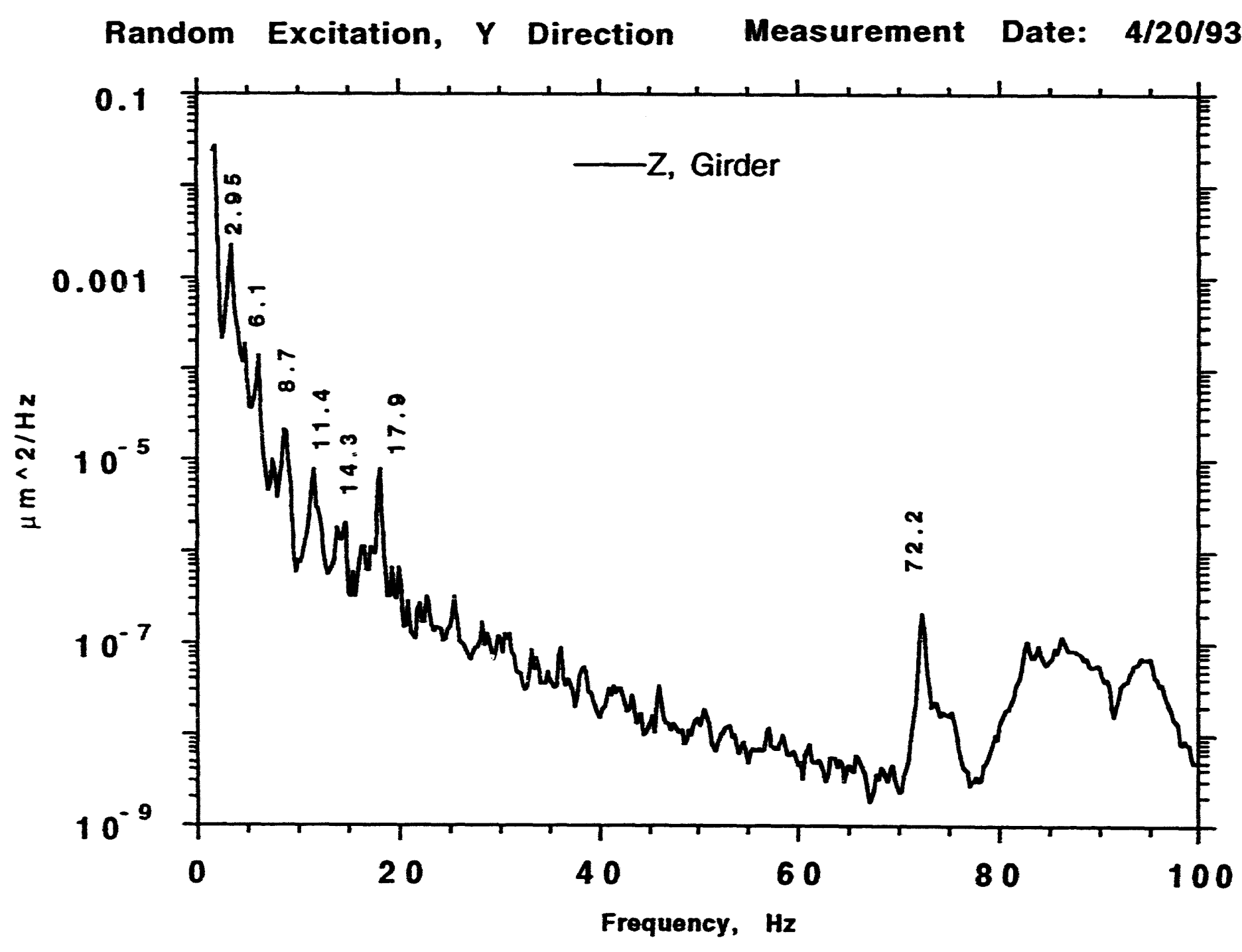




\section{SYNCHROTRON GIRDER RESPONSE}

Girder \# B2C61

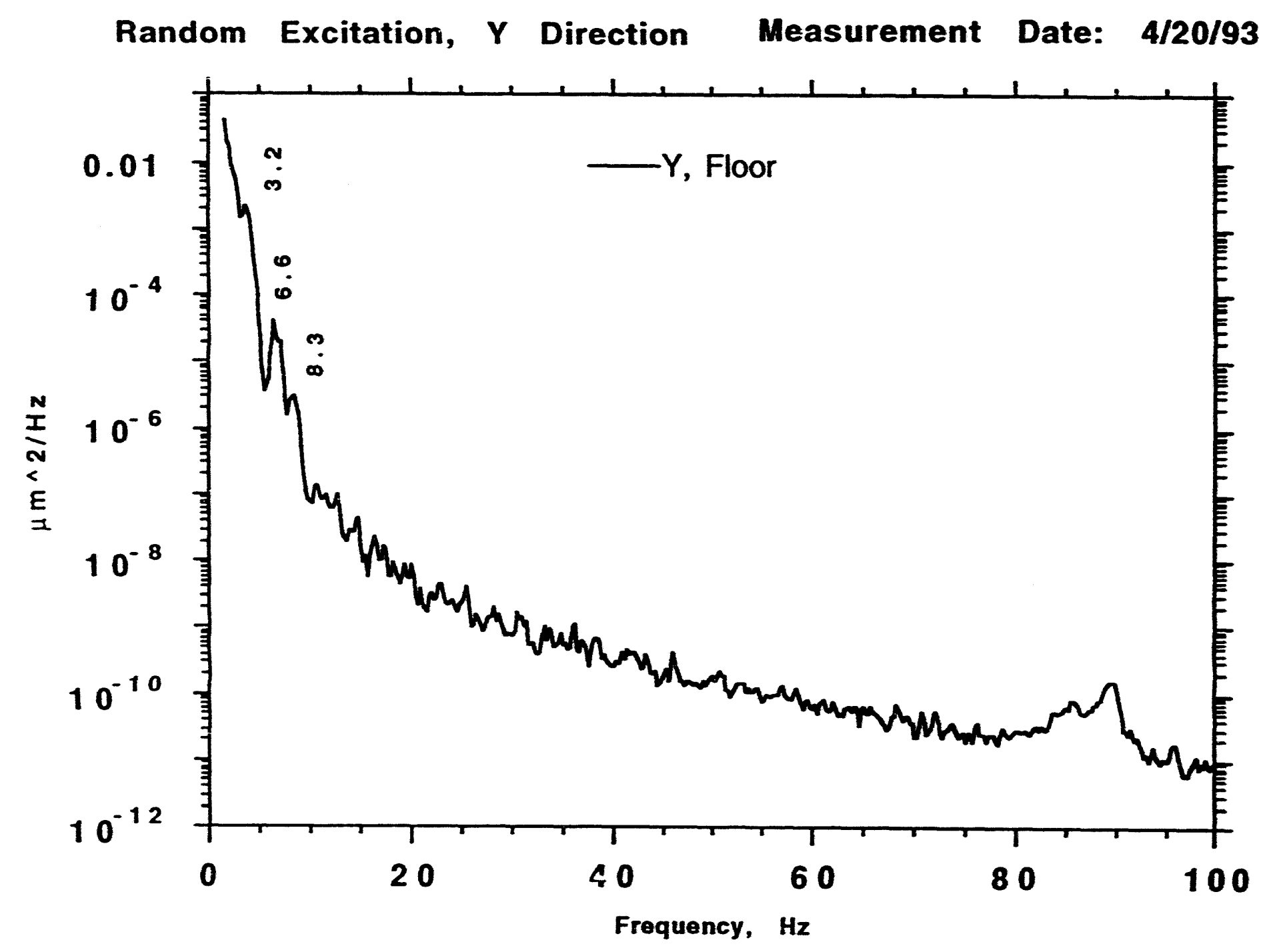




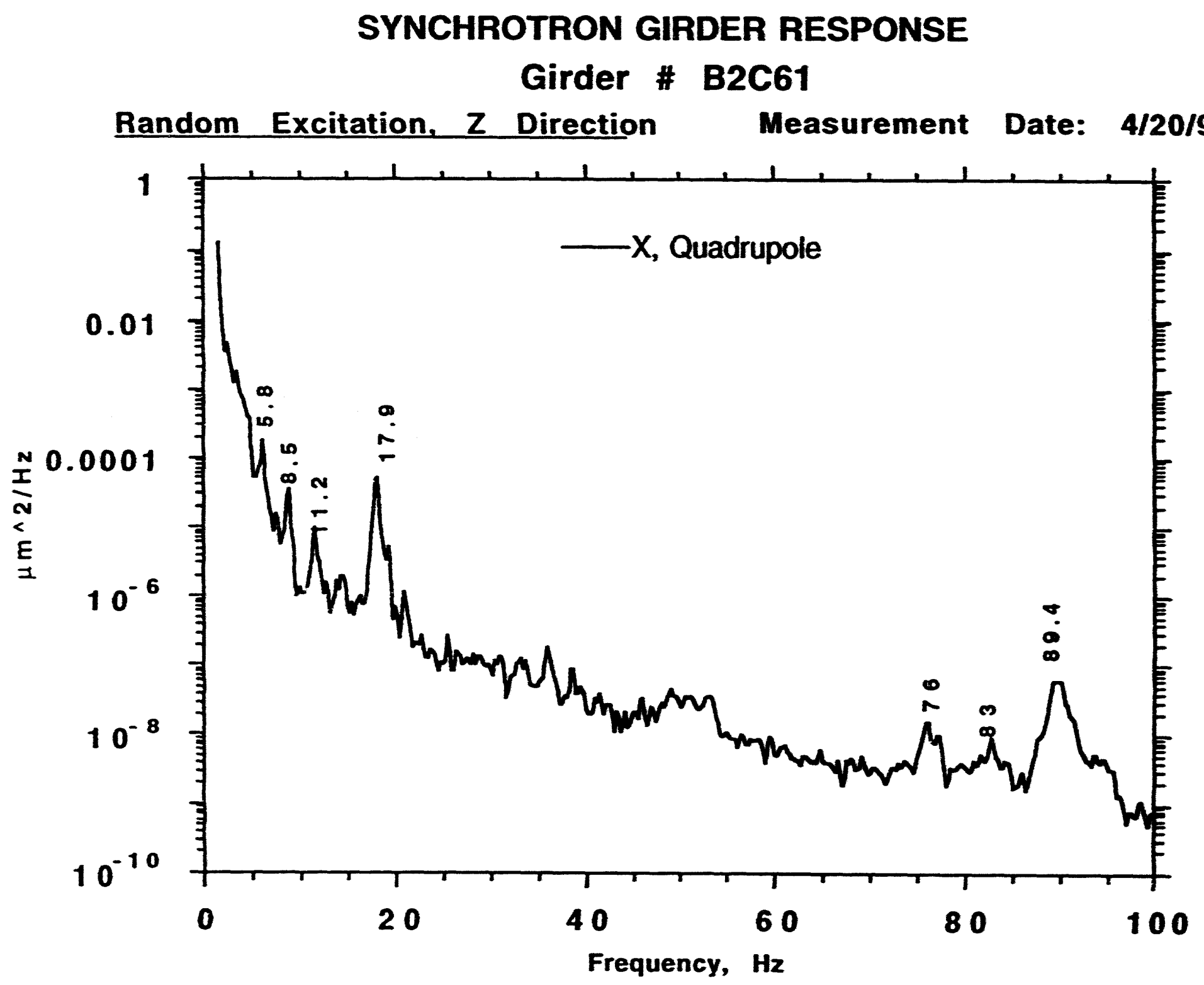




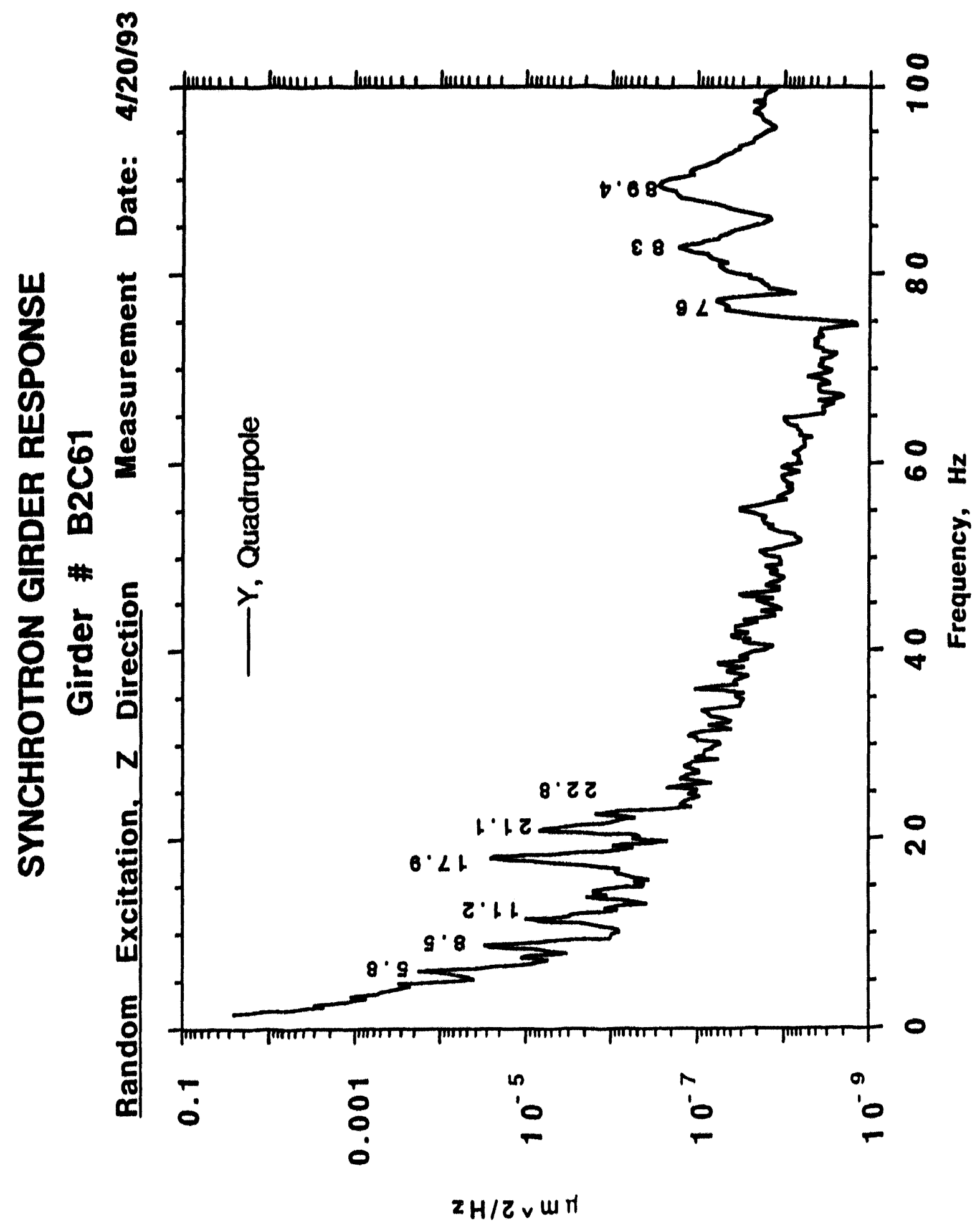




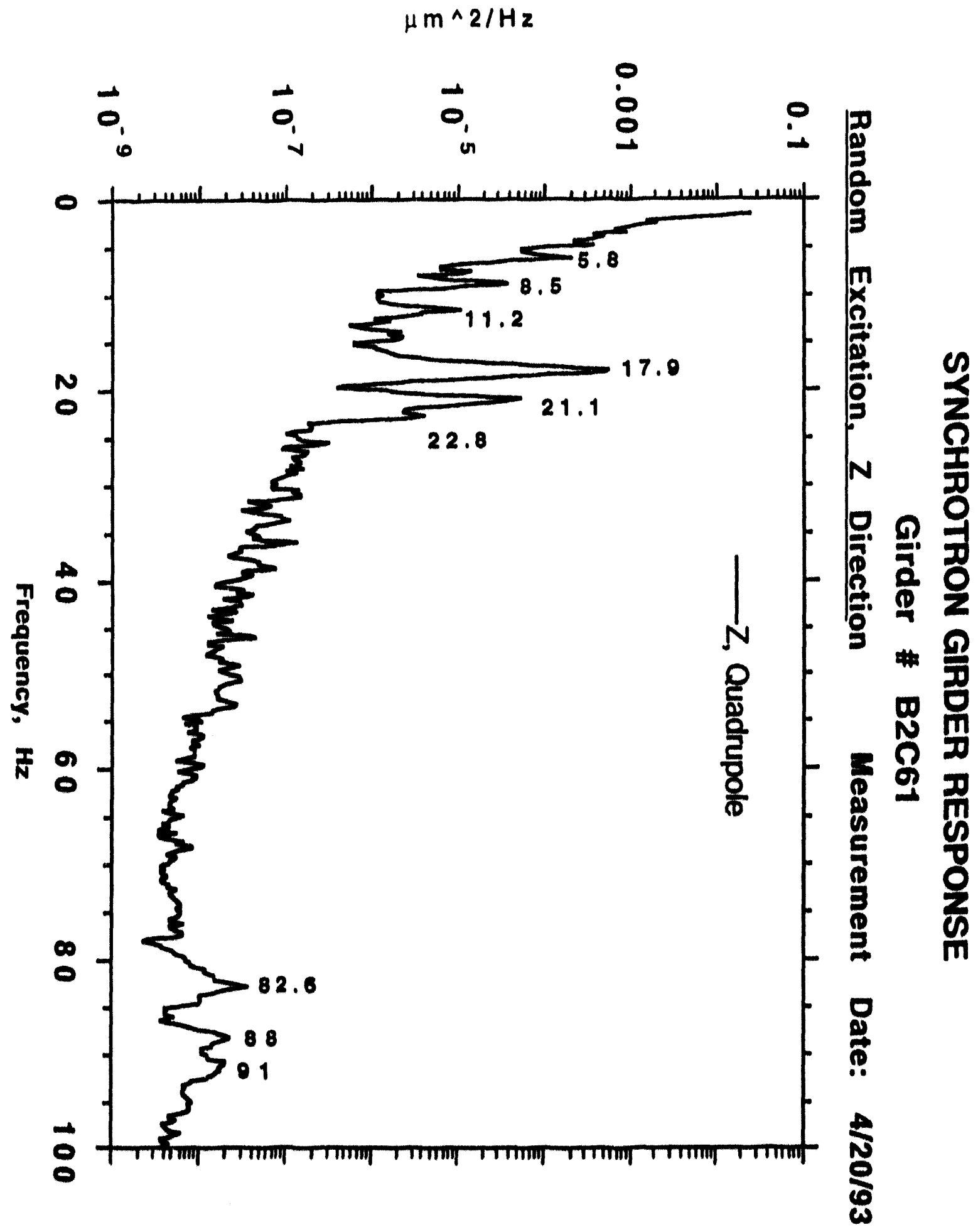




\section{SYNCHROTRON GIRDER RESPONSE}

\section{Girder \# B2C61}

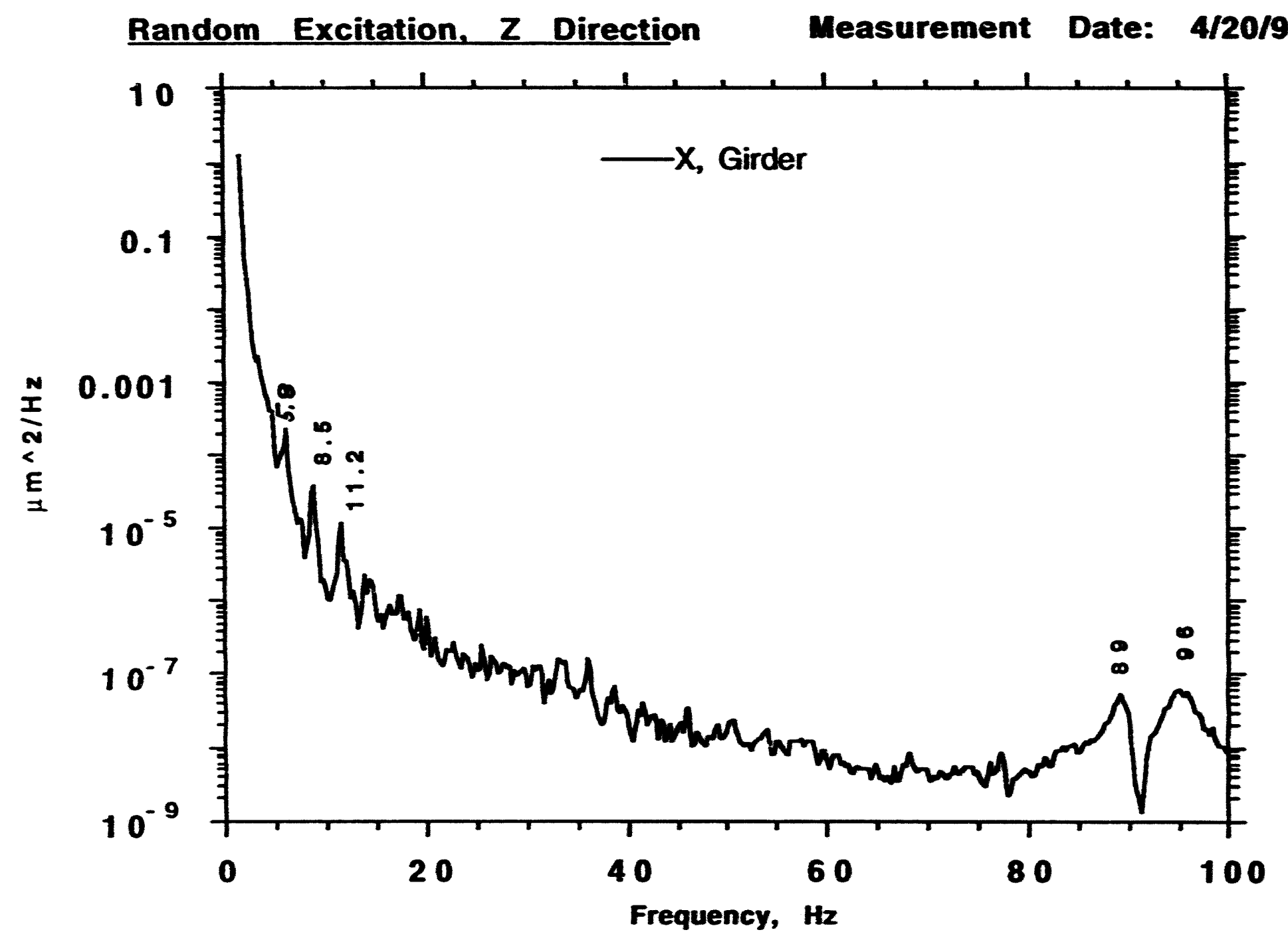




\section{SYNCHROTRON GIRDER RESPONSE}

\section{Girder \# B2C61}

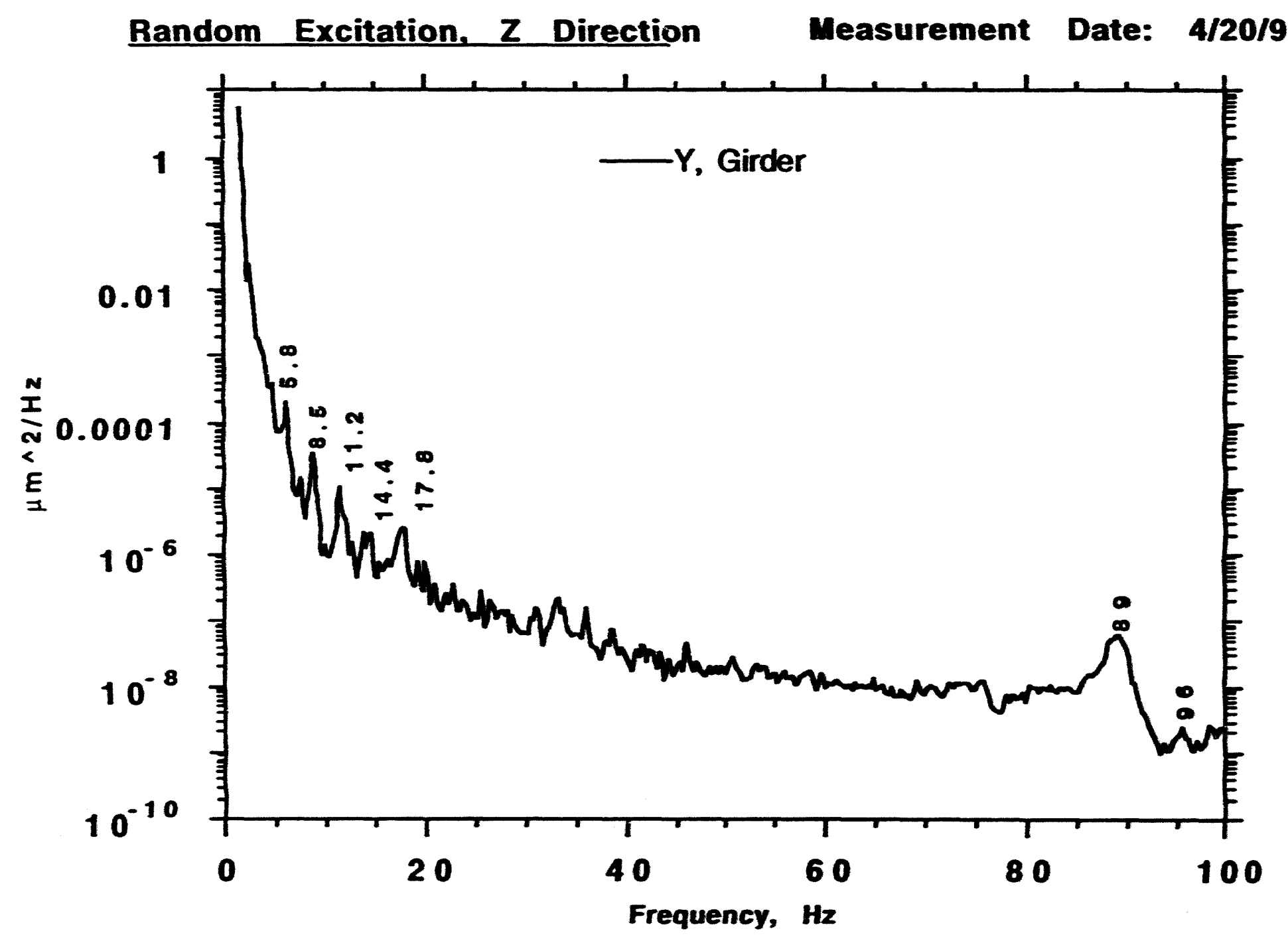




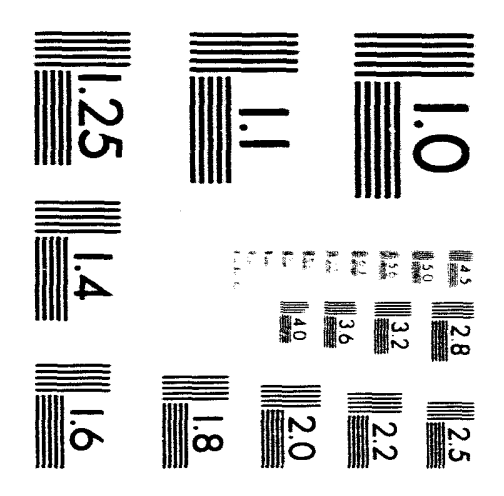



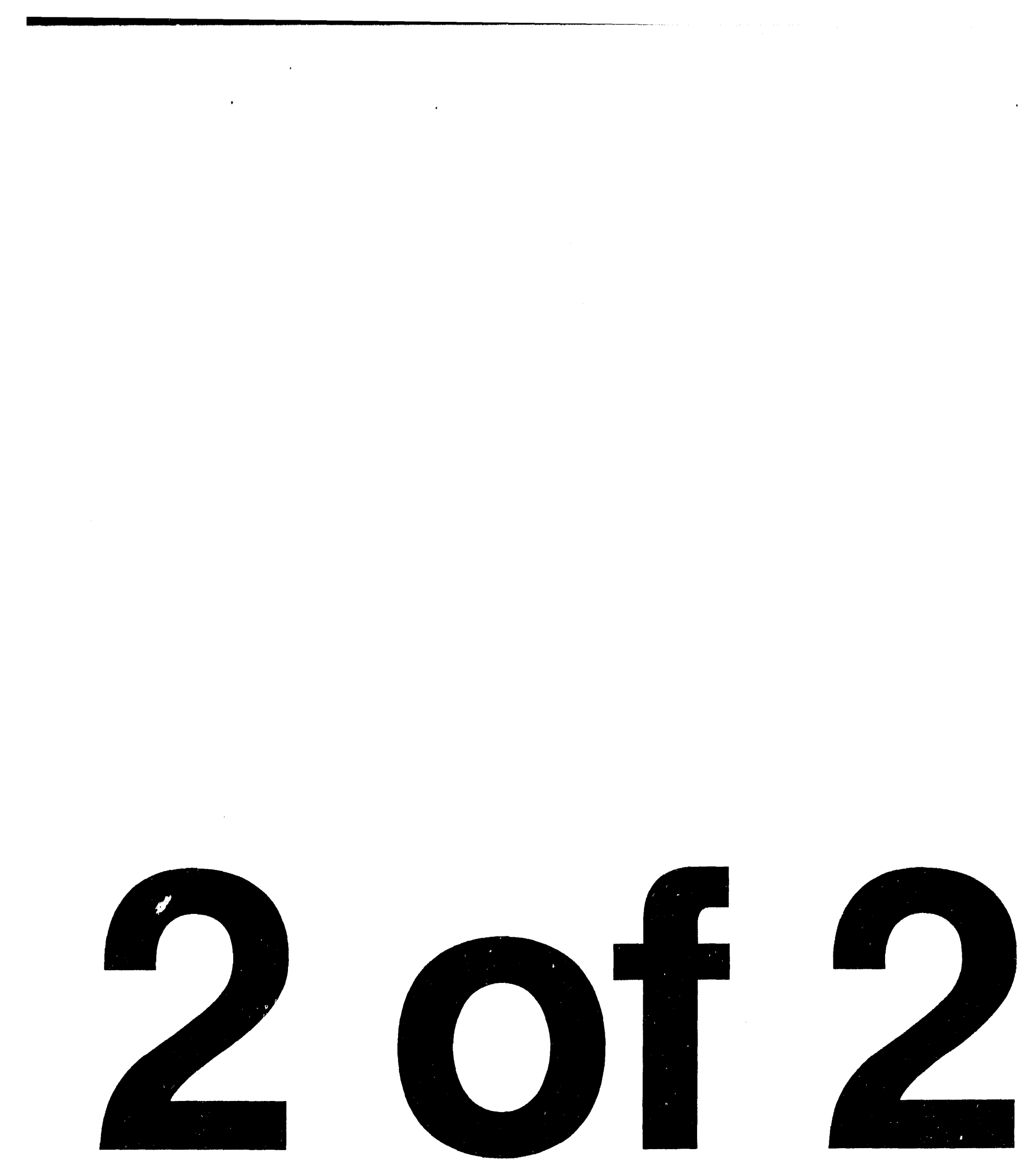


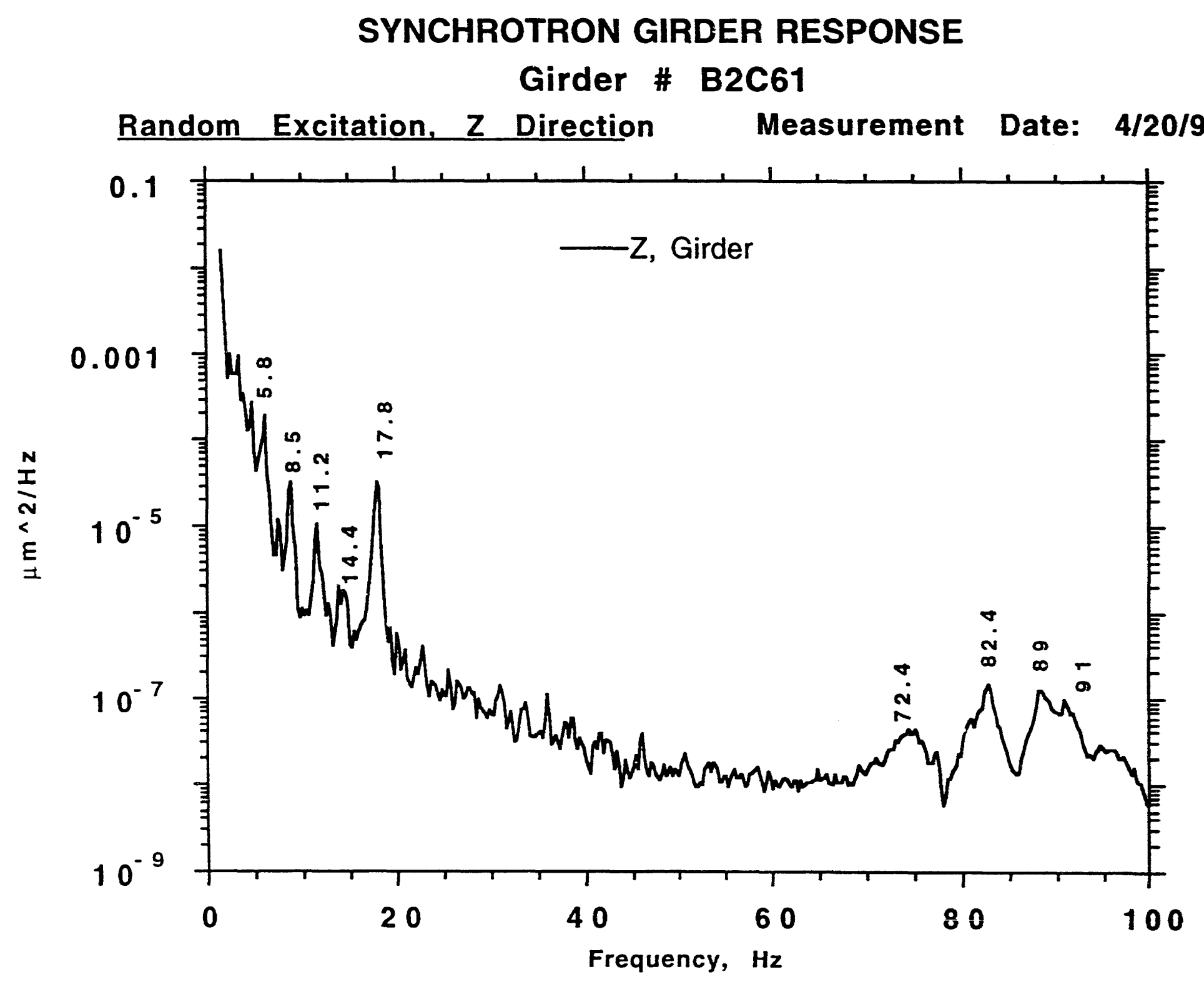




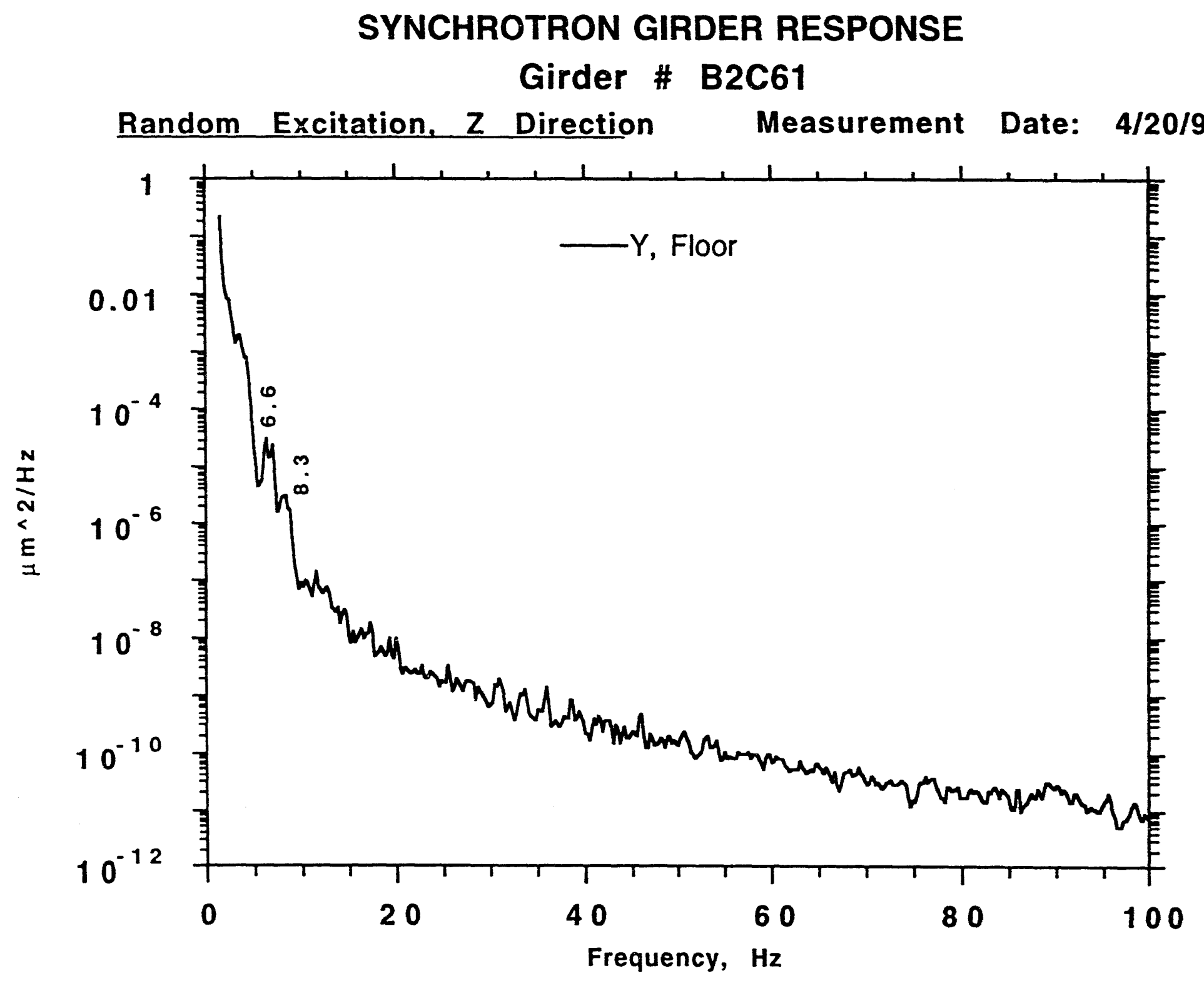




\section{SYNCHROTRON GIRDER RESPONSE}

\section{Girder \# B2C61}

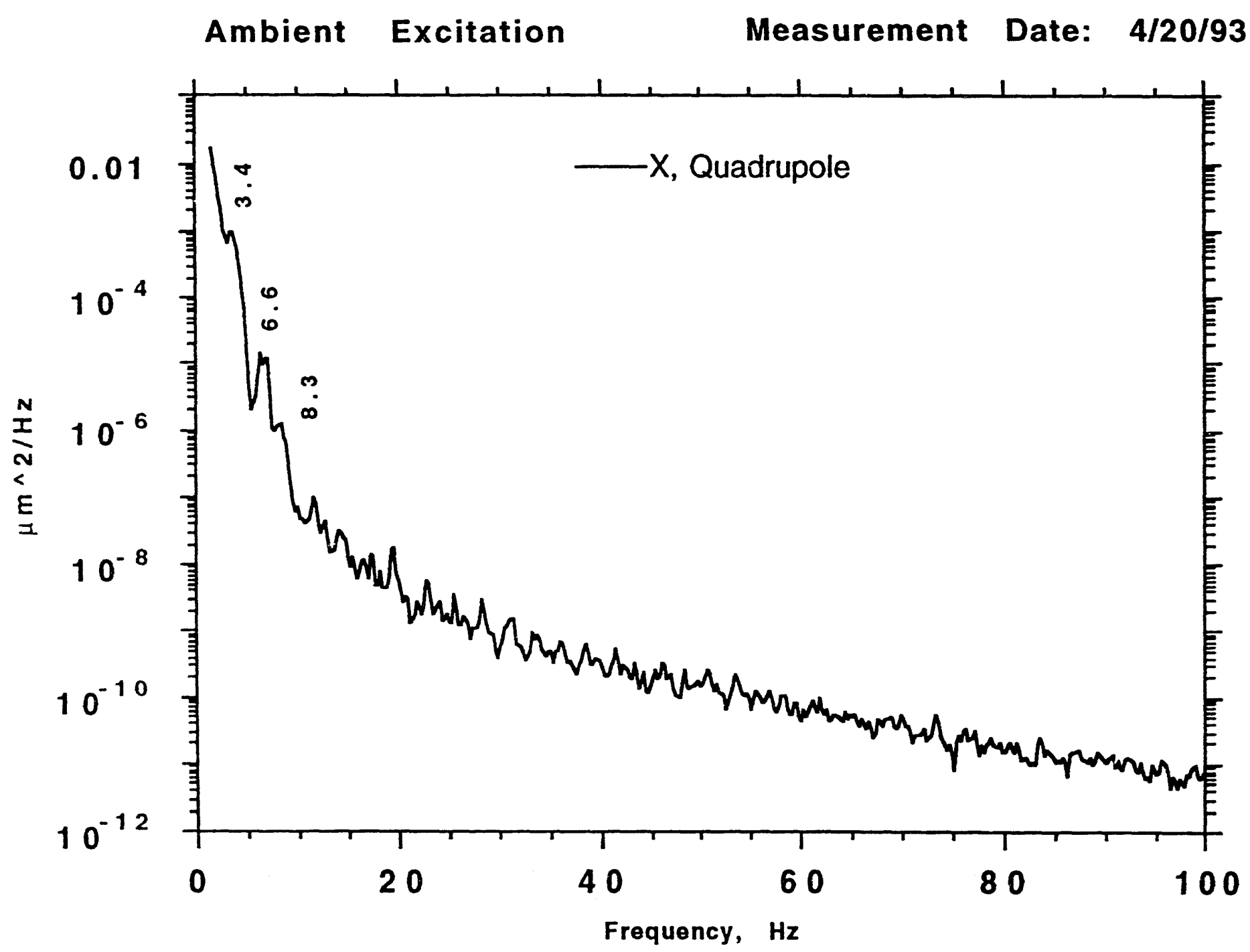




\section{SYNCHROTRON GIRDER RESPONSE}

Girder \# B2C61

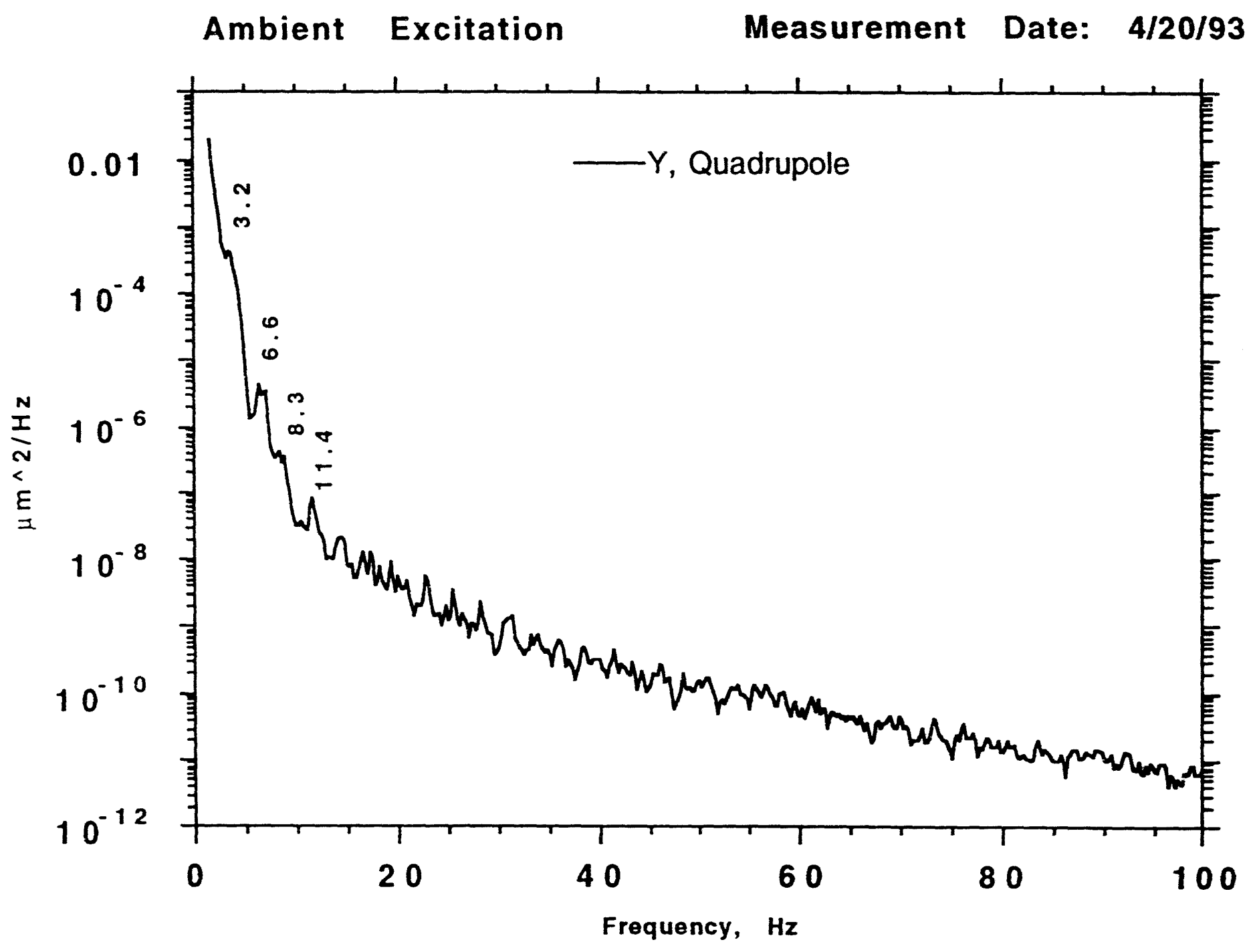




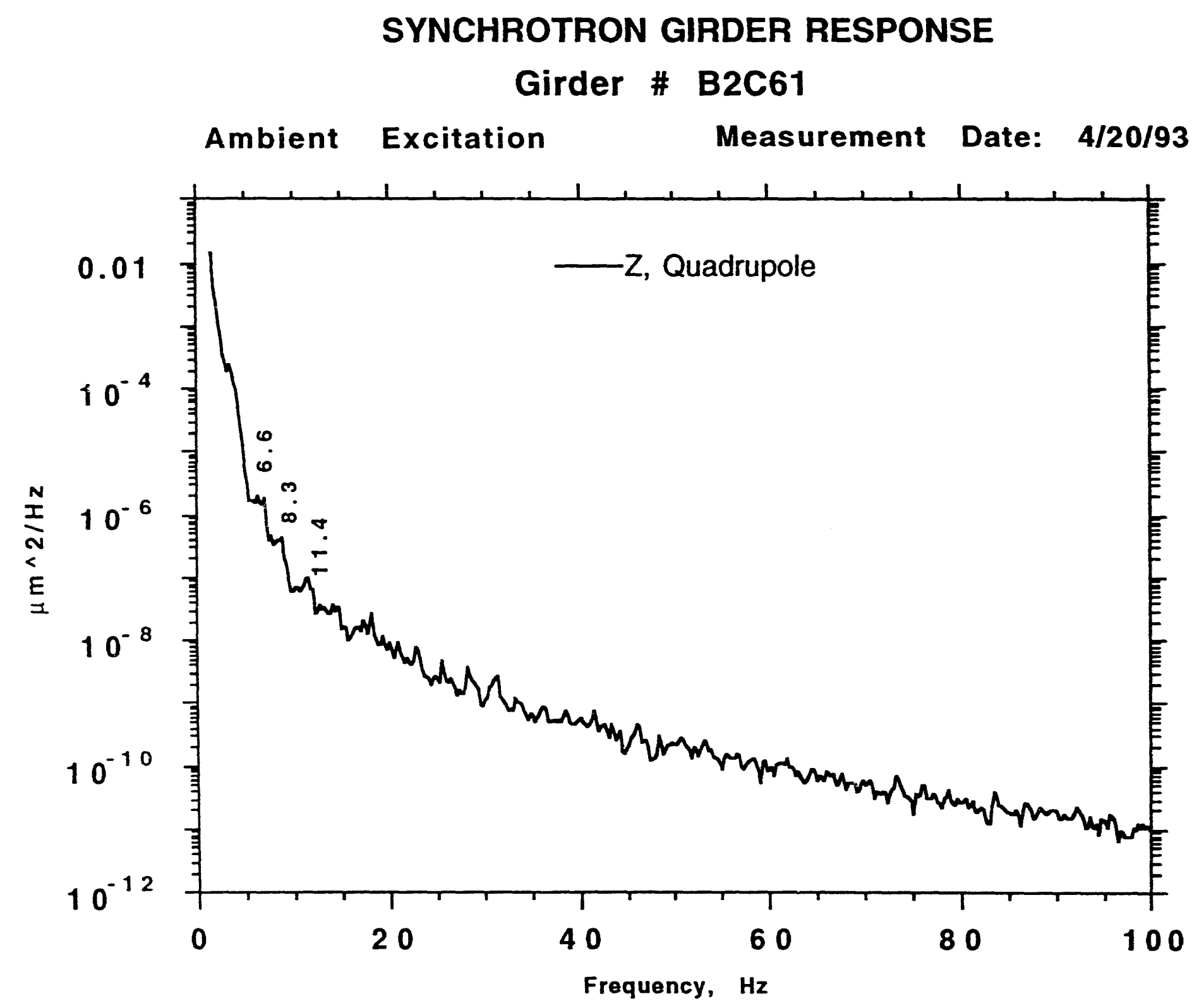




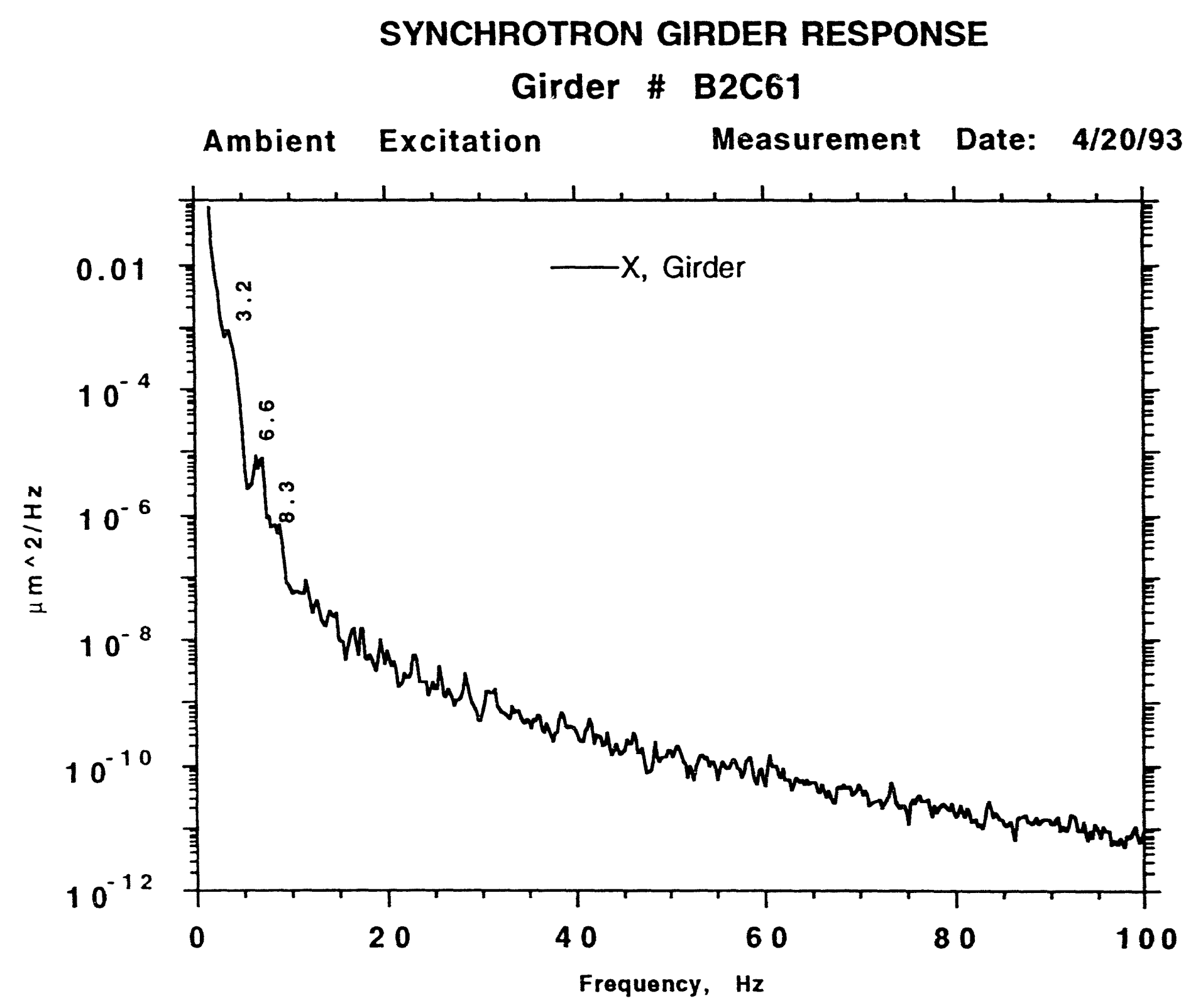




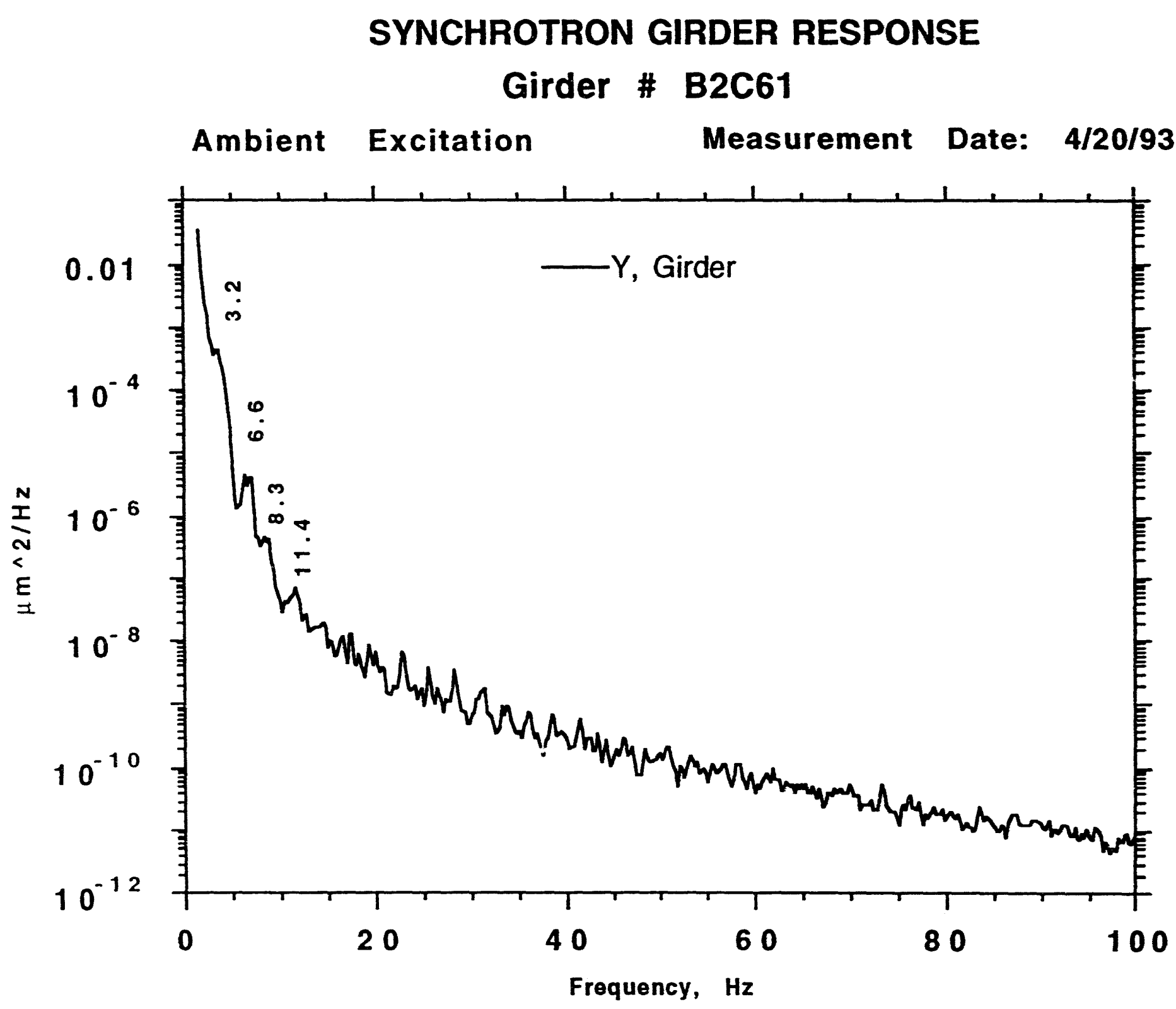




\section{SYNCHROTRON GIRDER RESPONSE}

Girder \# B2C61

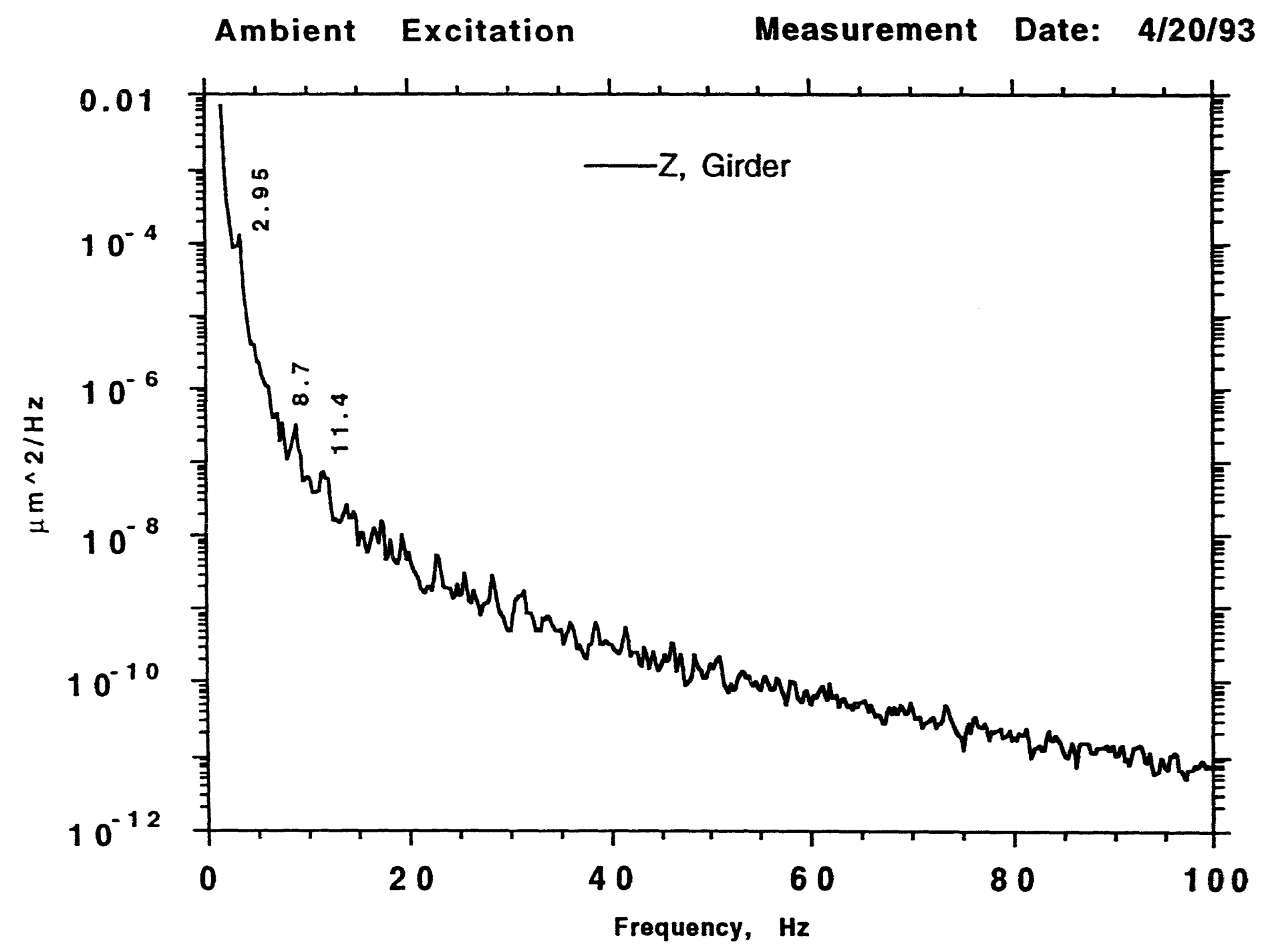




\section{SYNCHROTRON GIRDER RESPONSE}

\section{Girder \# B2C61}

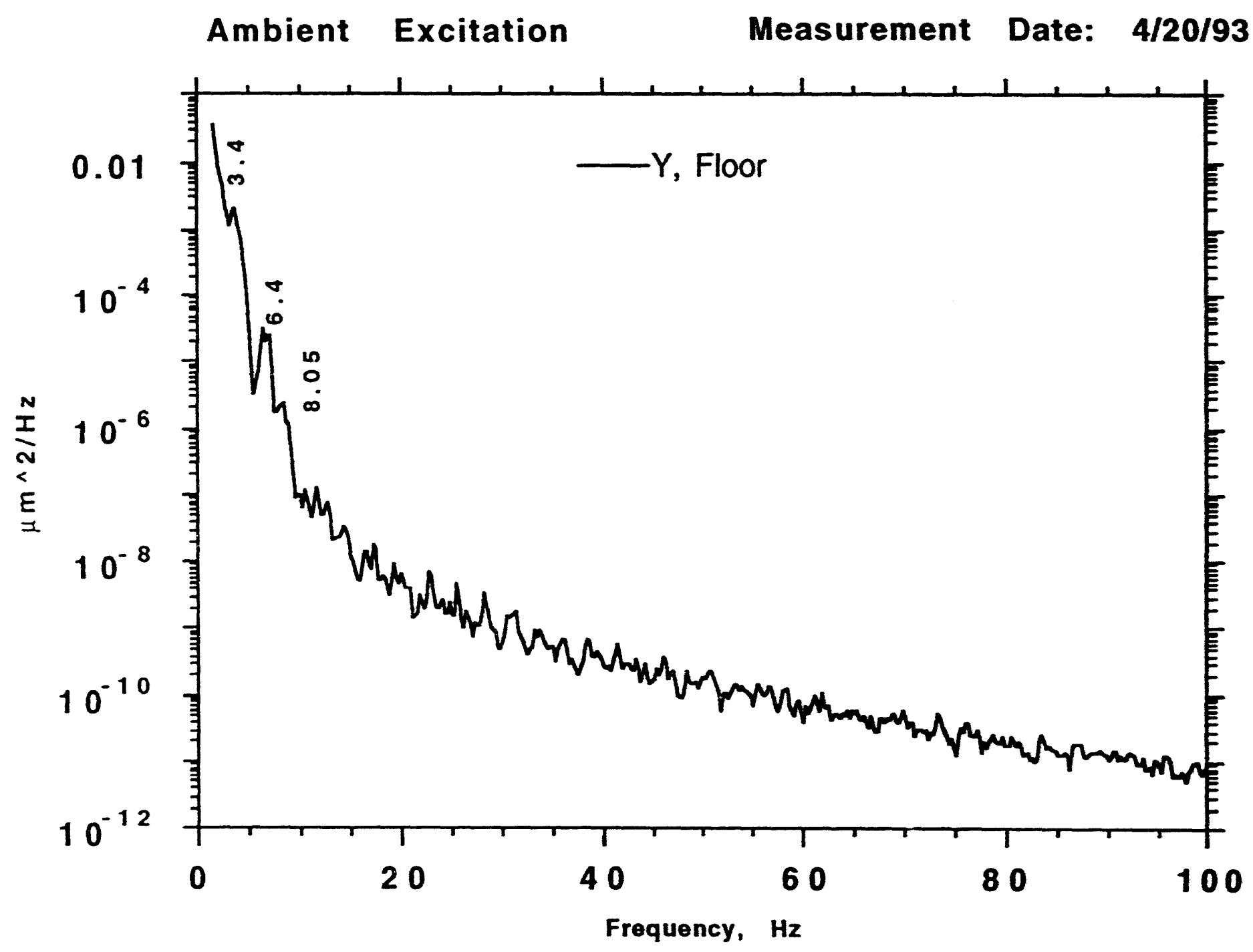


Appendix D: Sextupole magnet response 


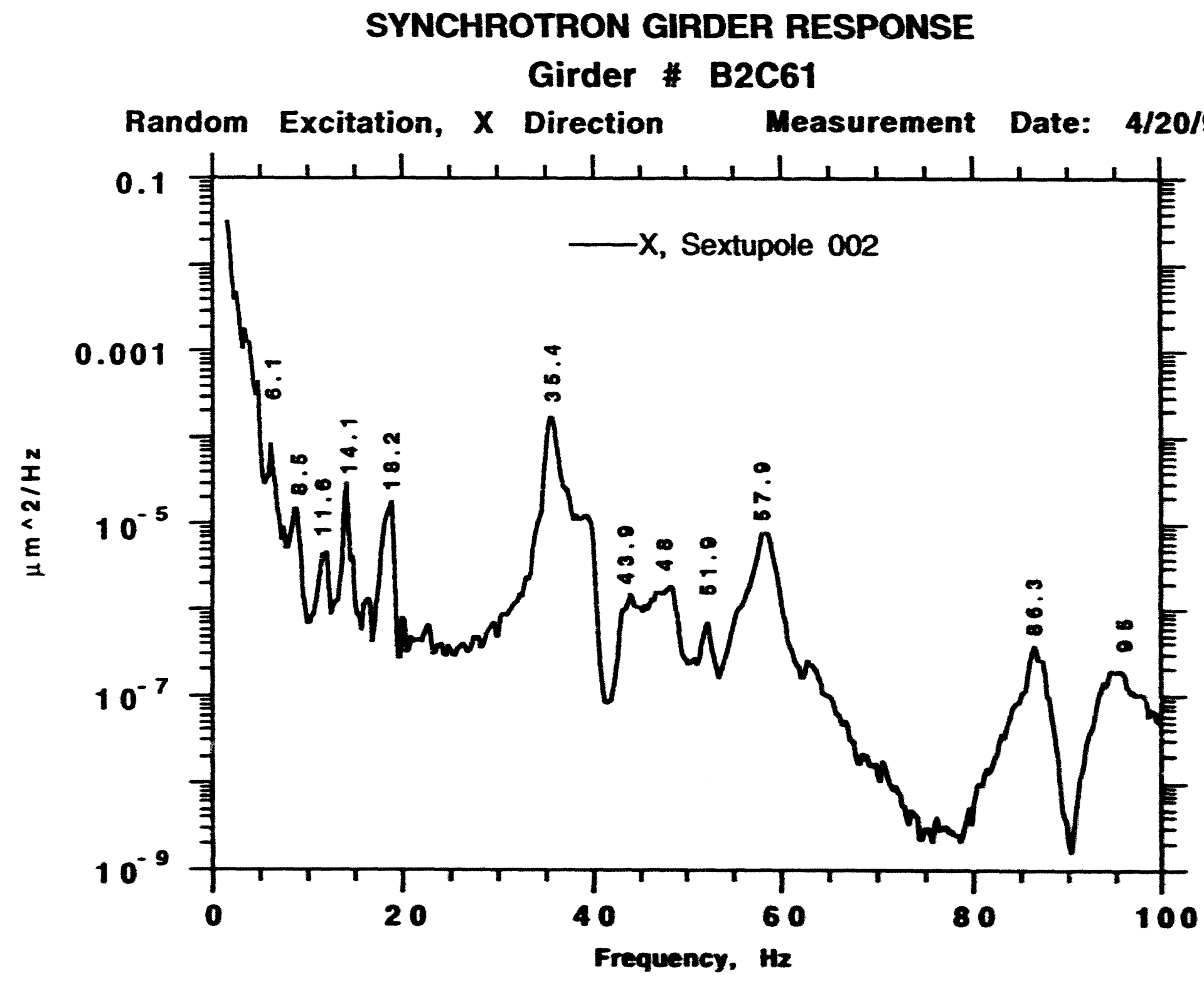




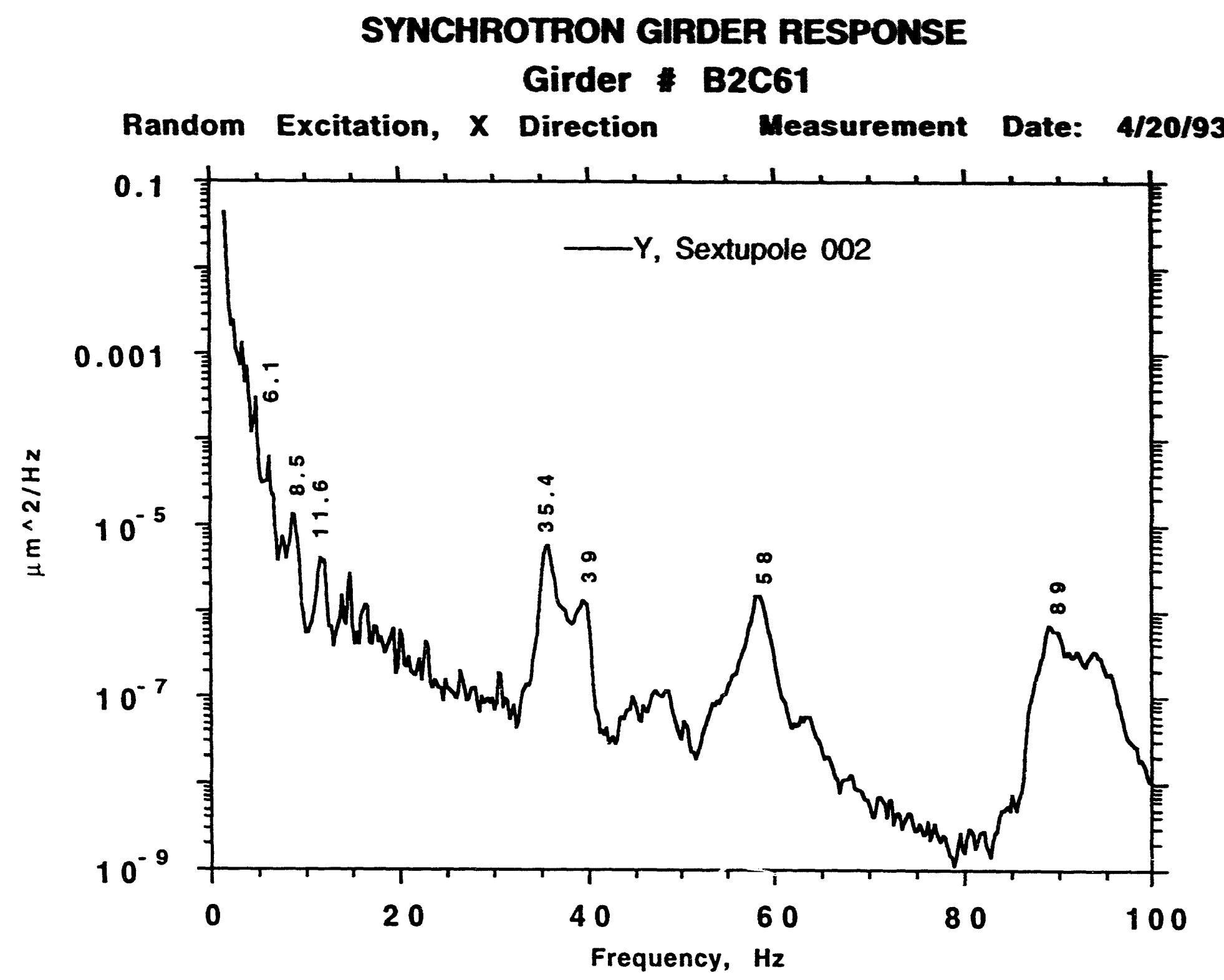

0 


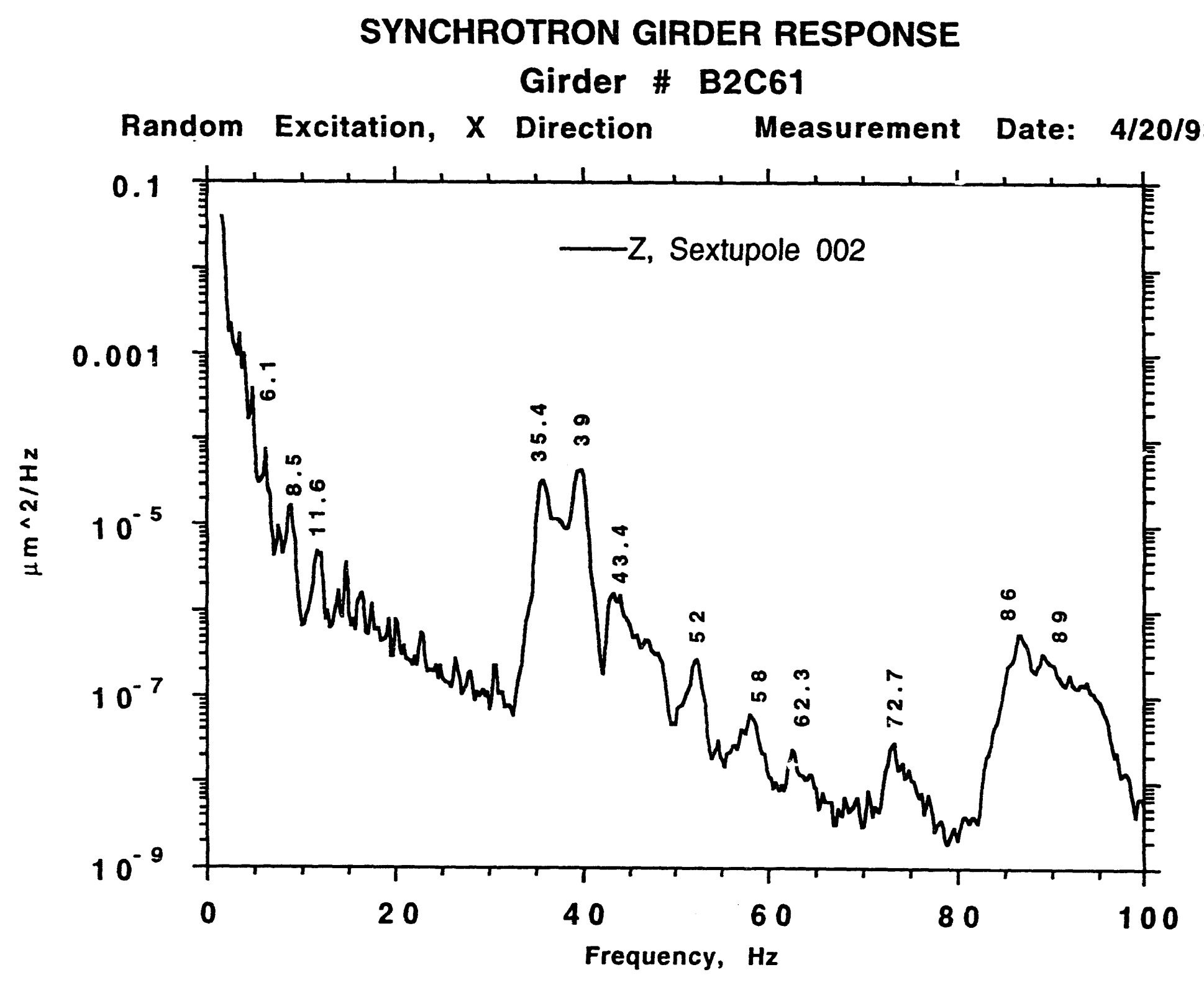




\section{SYNCHROTRON GIRDER RESPONSE}

Girder \# B2C61

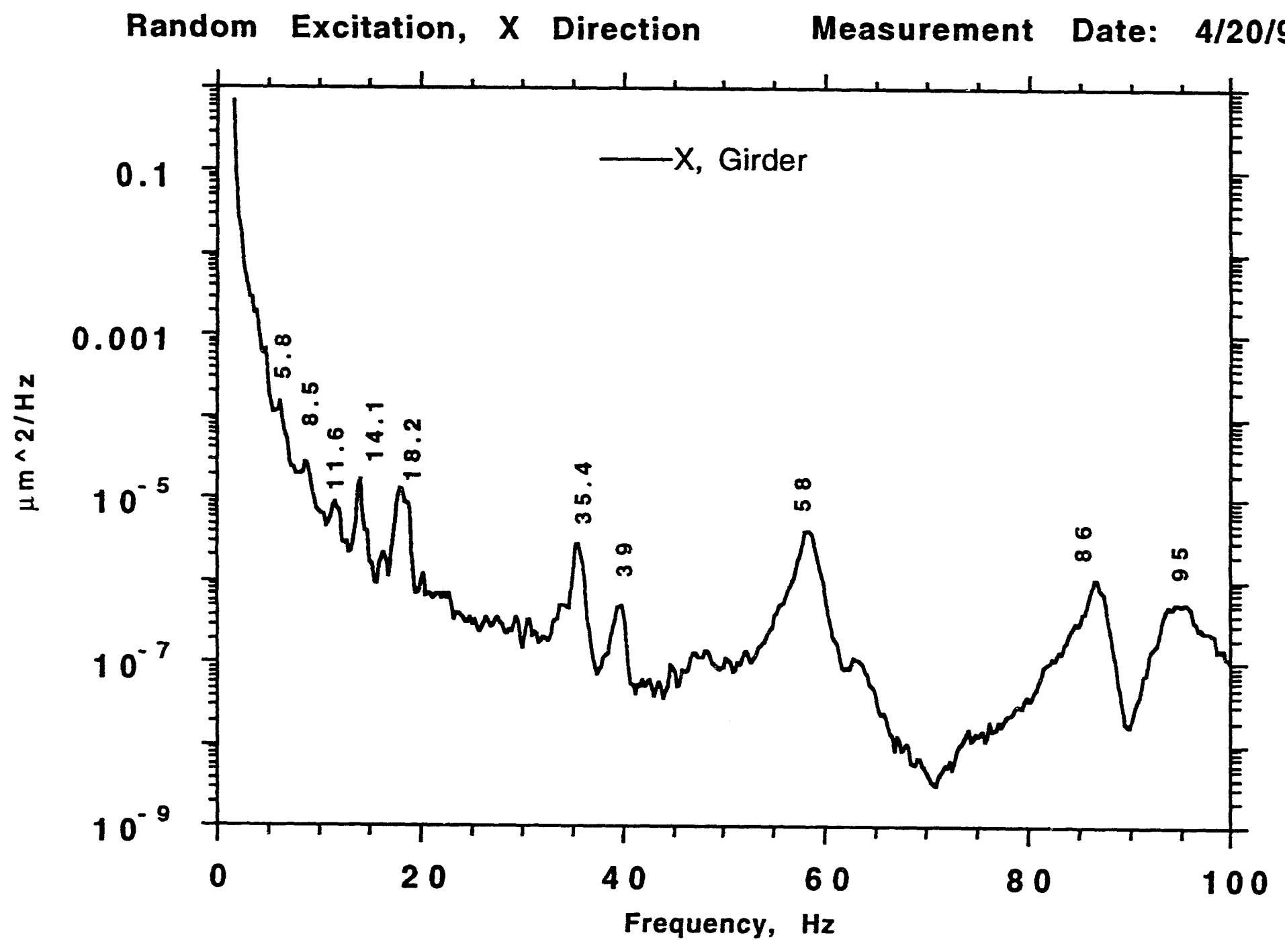




\section{SYNCHROTRON GIRDER RESPONSE}

\section{Girder \# B2C61}

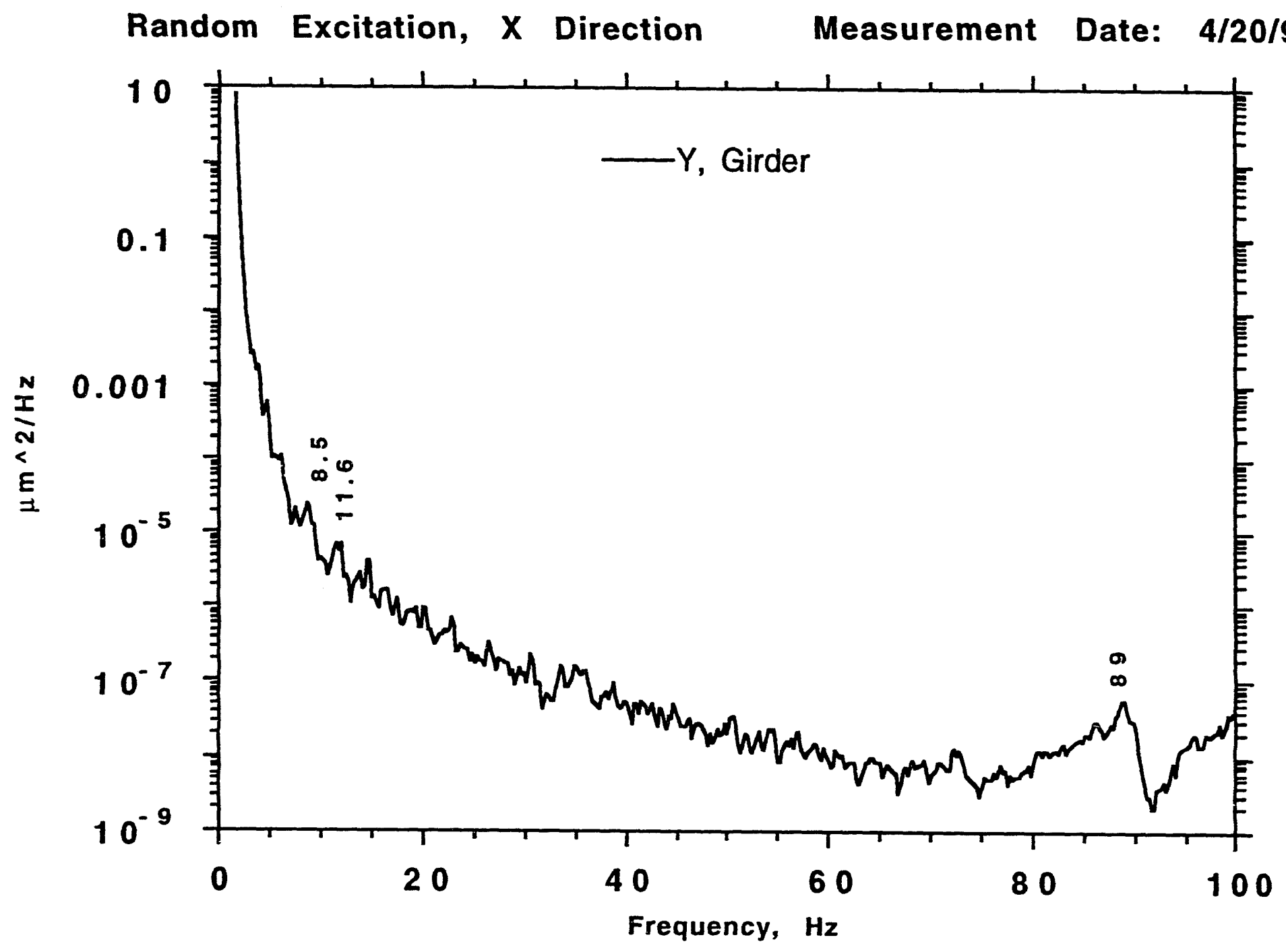




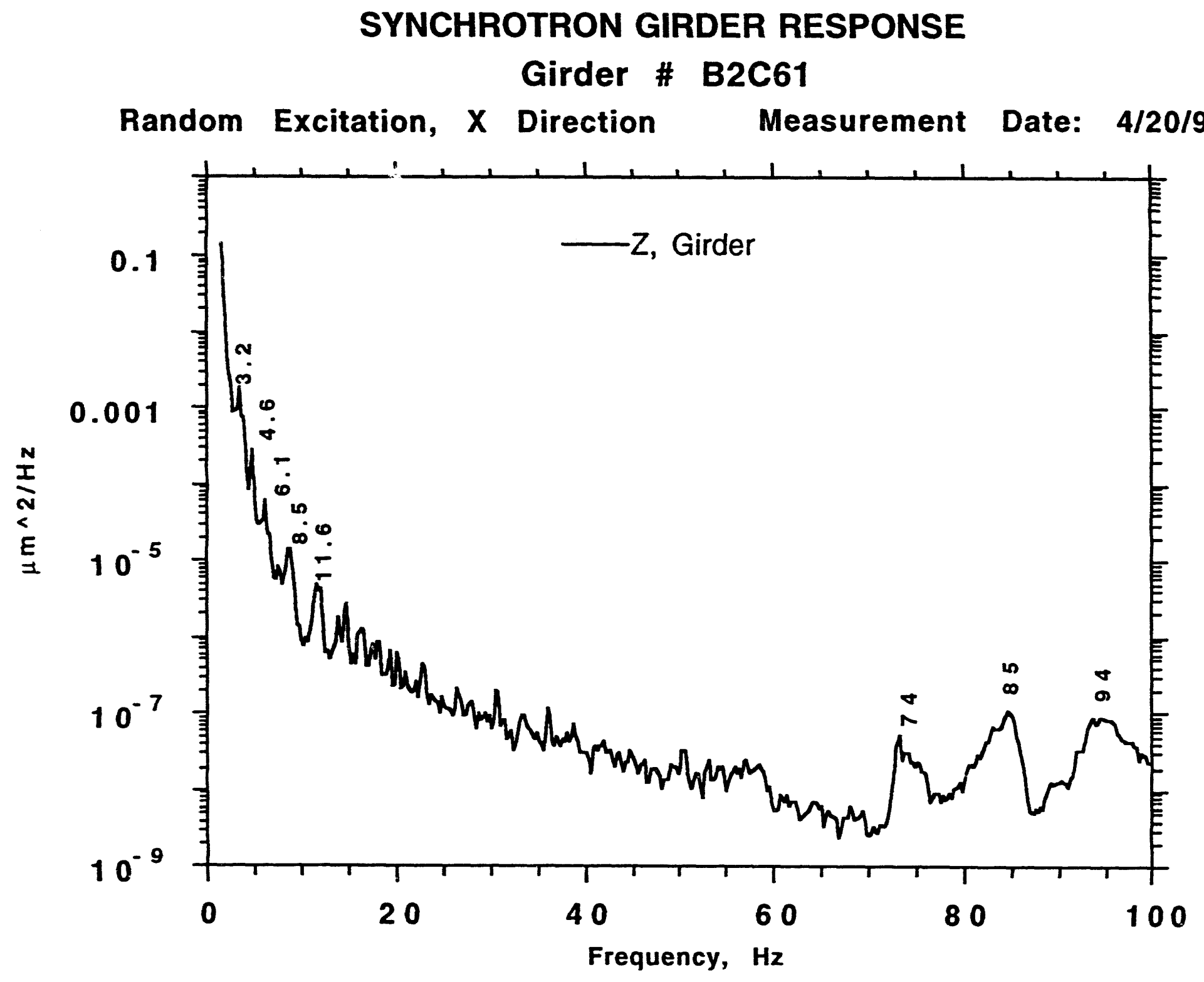




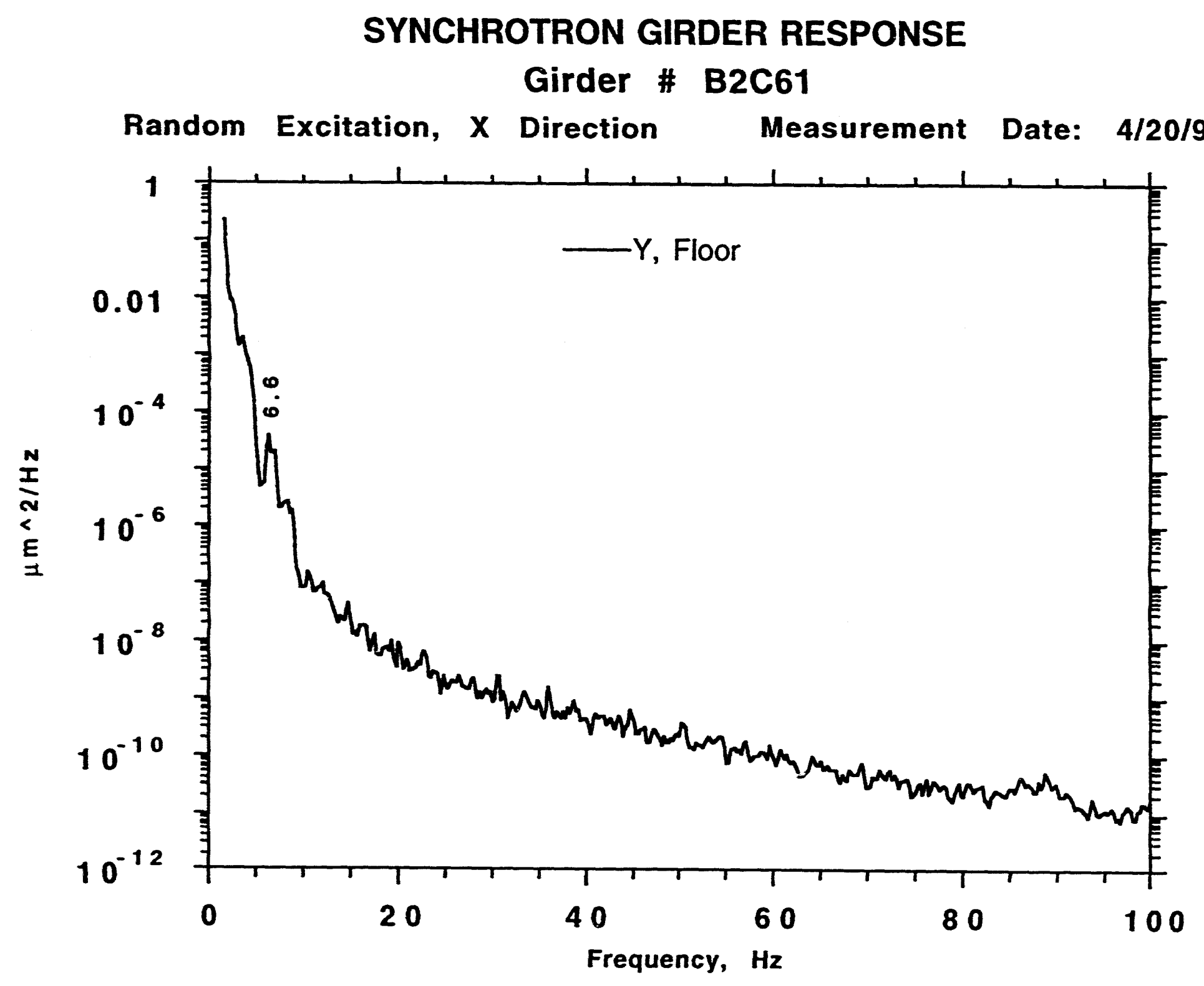




\section{SYNCHROTRON GIRDER RESPONSE}

\section{Girder \# B2C61}

Random Excitation, $Y$ Direction Measurement Date: 4/20/93

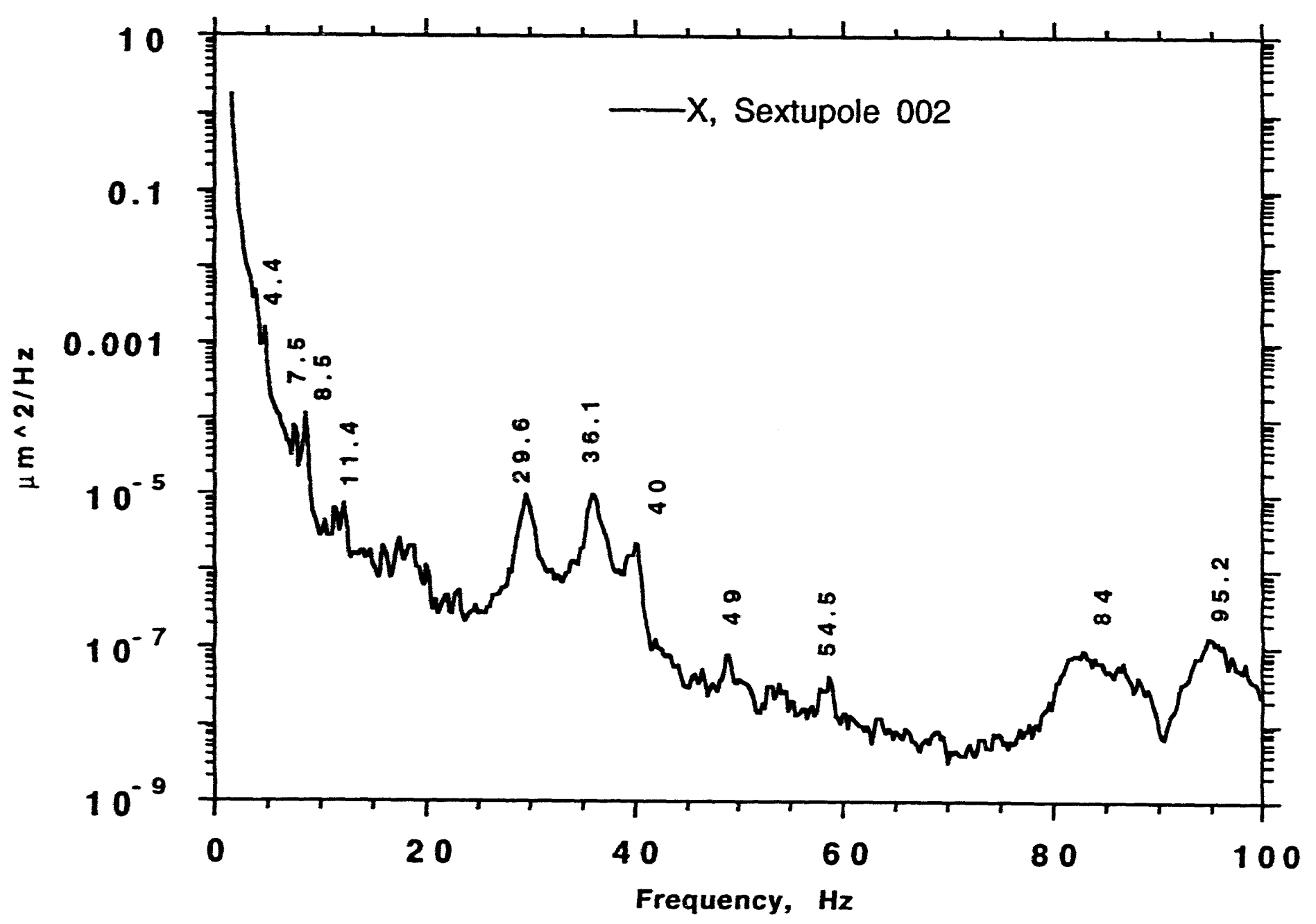




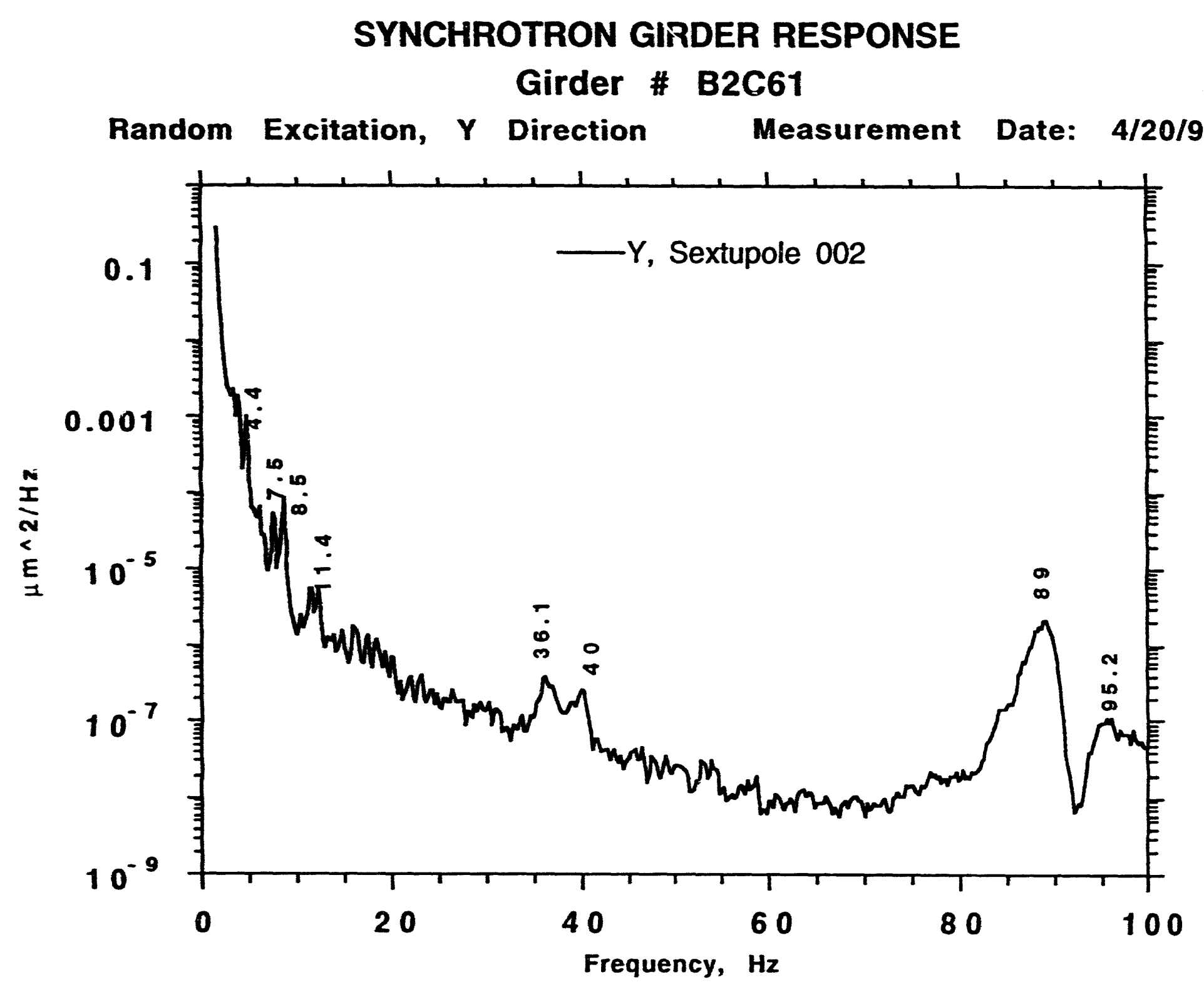

b 


\section{SYNCHROTRON GIRDER RESPONSE}

Girder \# B2C61

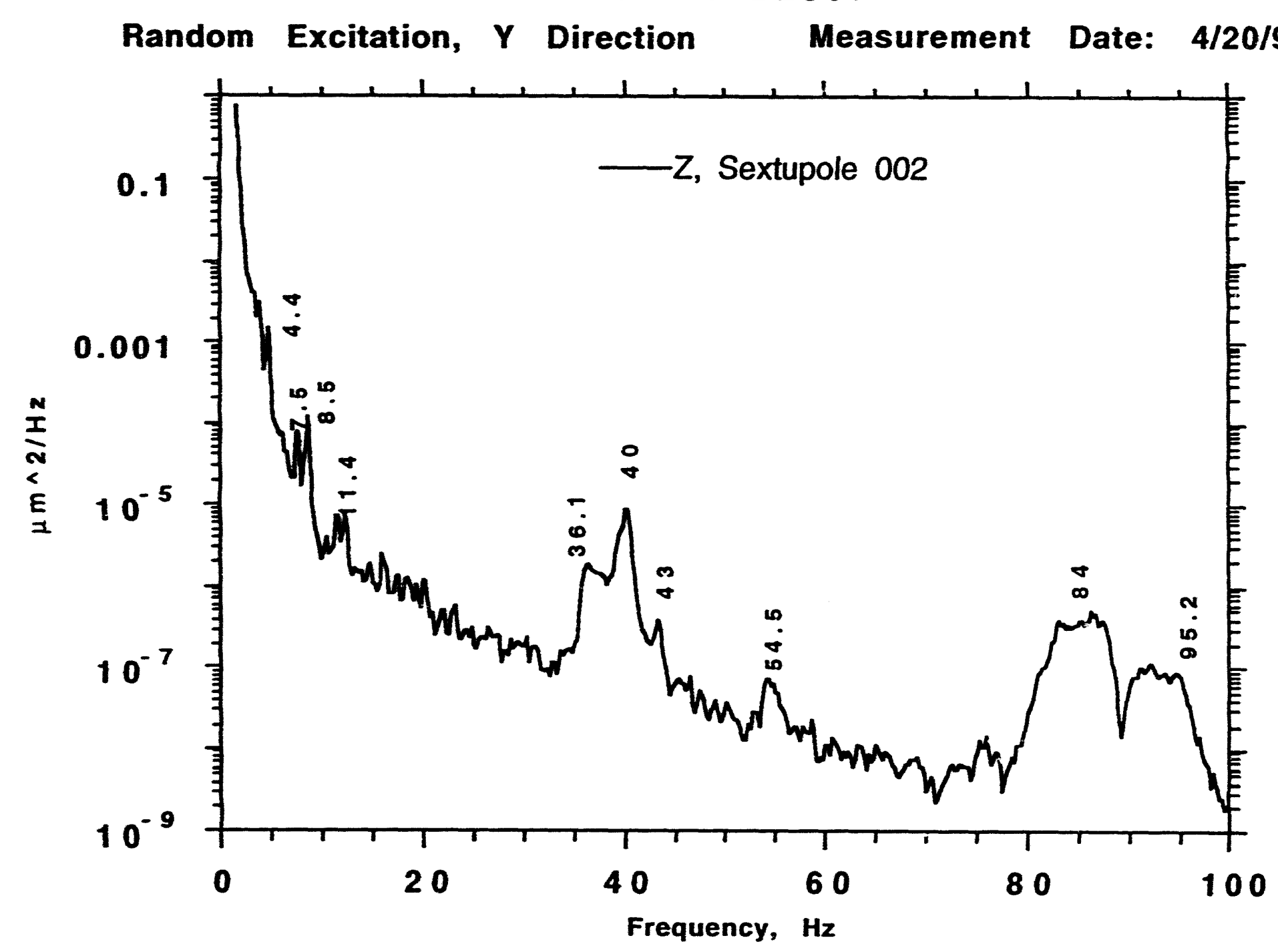




\section{SYNCHROTRON GIRDER RESPONSE}

\section{Girder \# B2C61}

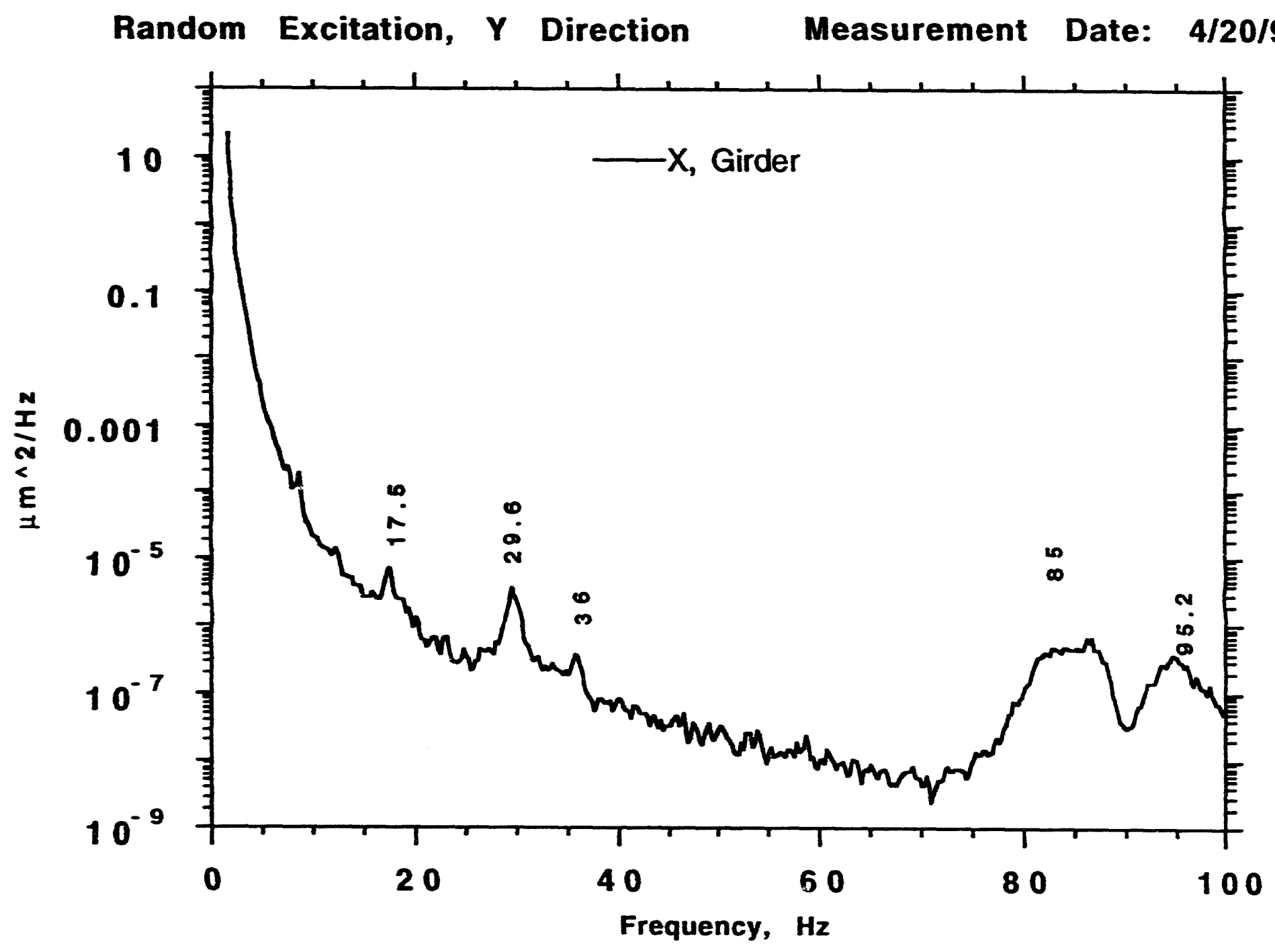




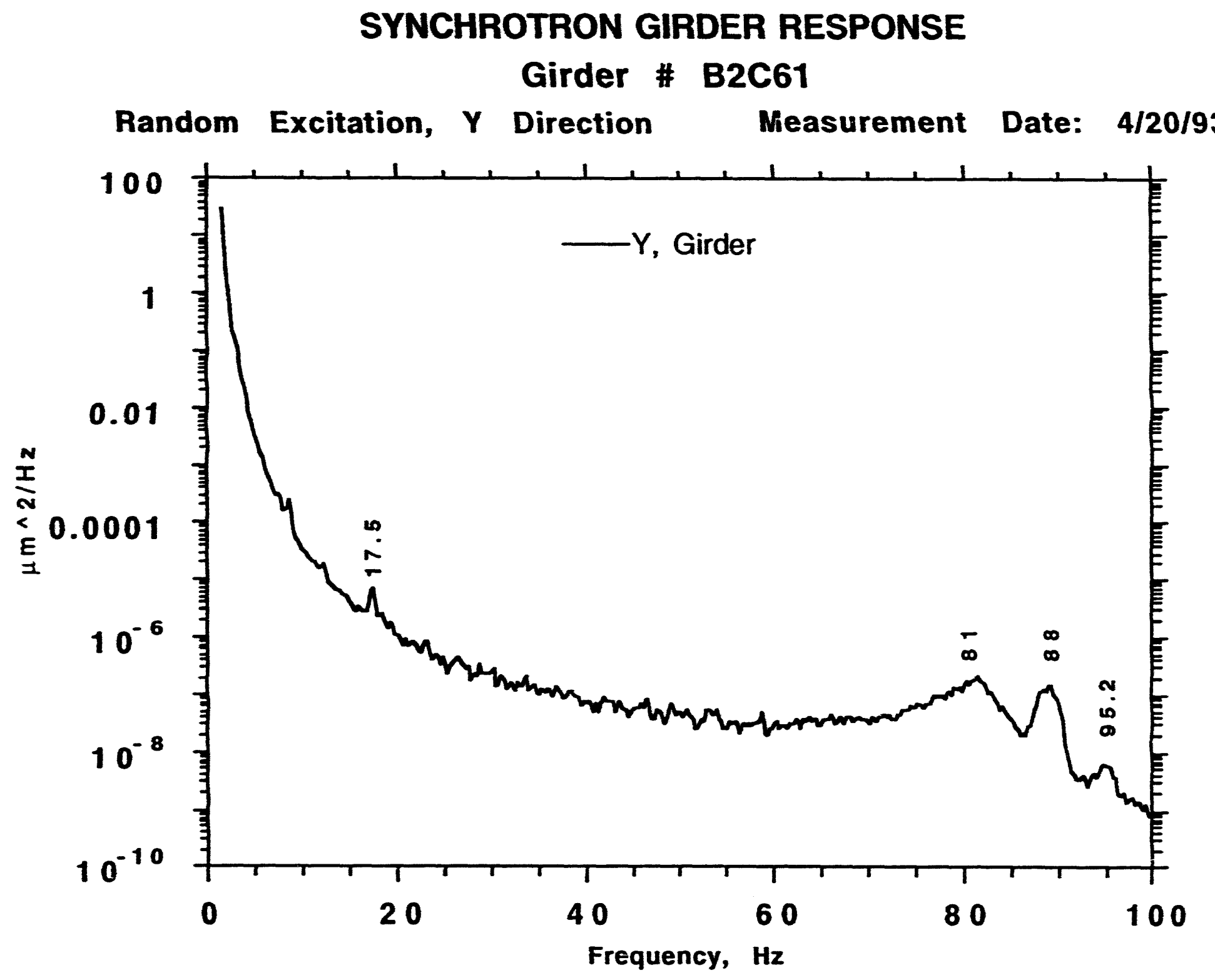




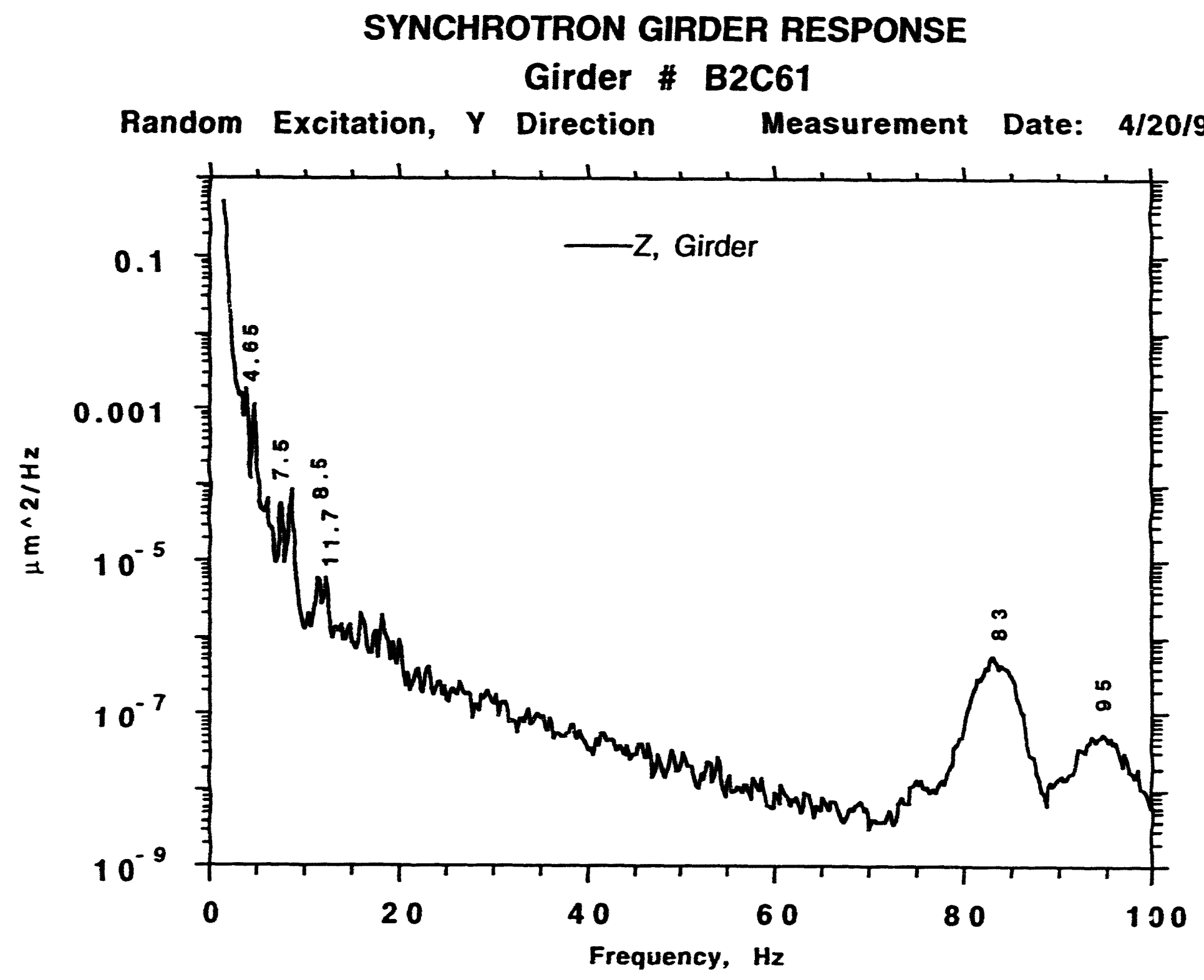




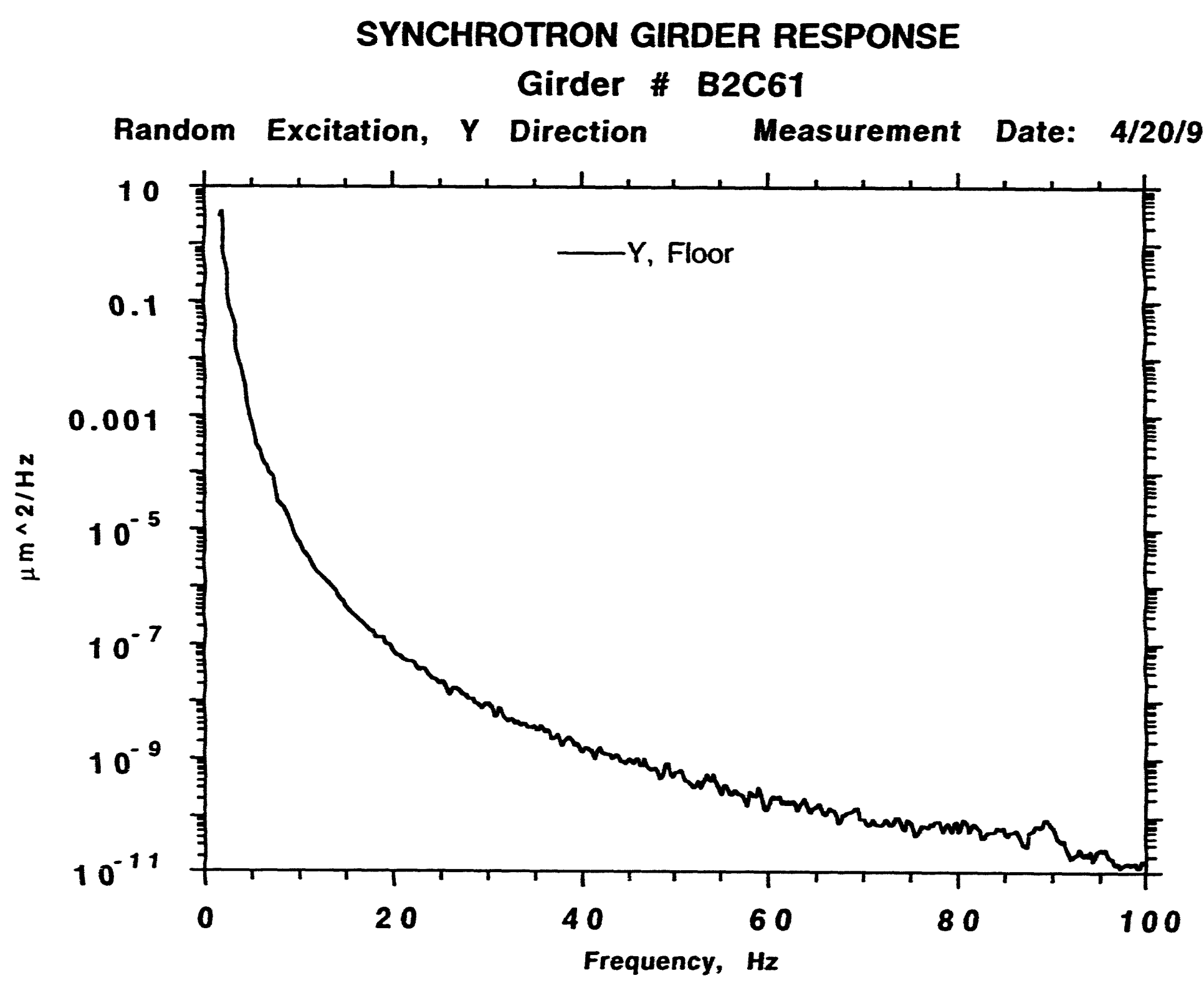




\section{SYNCHROTRON GIRDER RESPONSE \\ Girder \# B2C61}

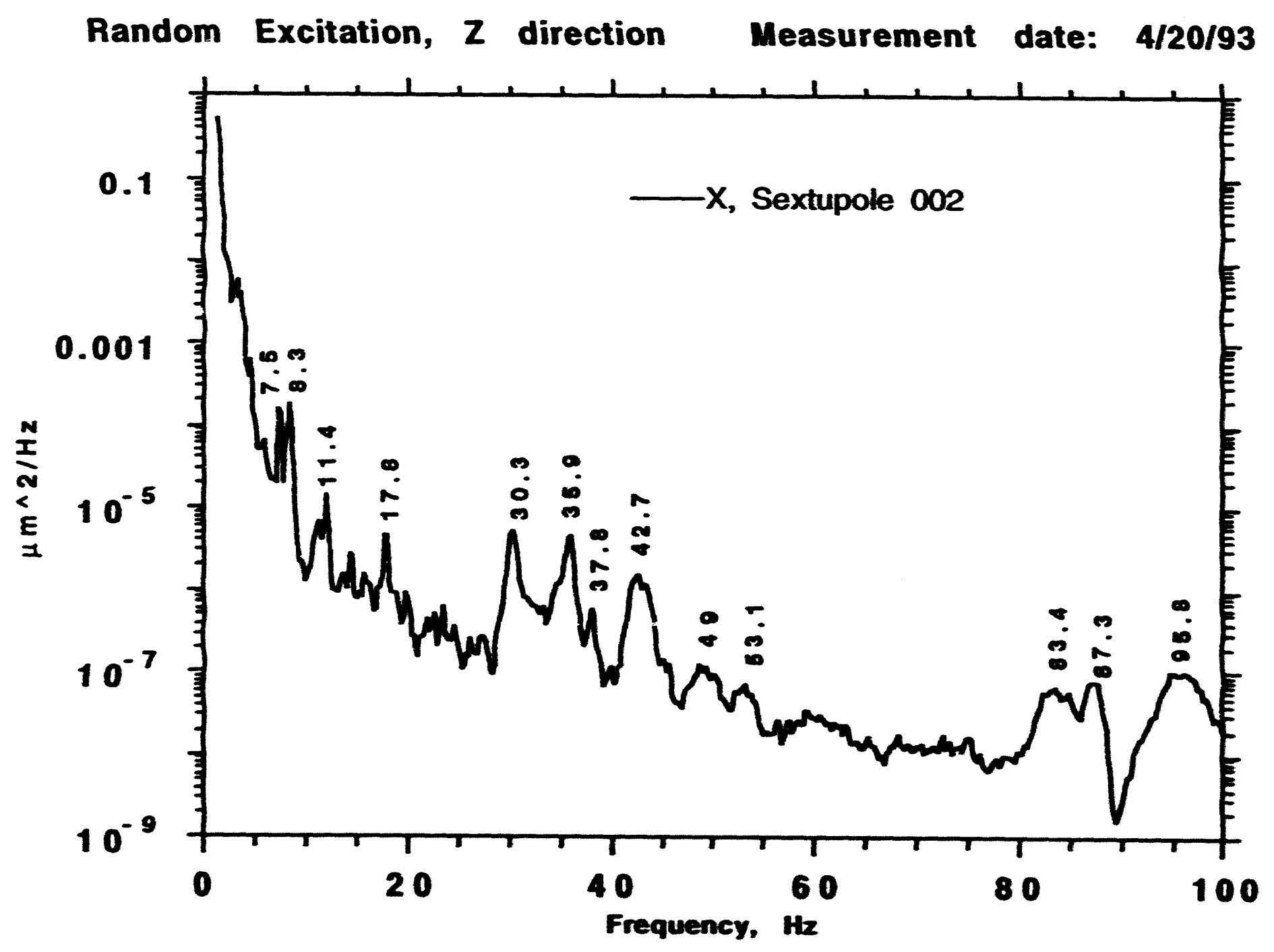

是 


\section{SYNCHROTRON GIRDER RESPONSE \\ Girder \# B2C61}

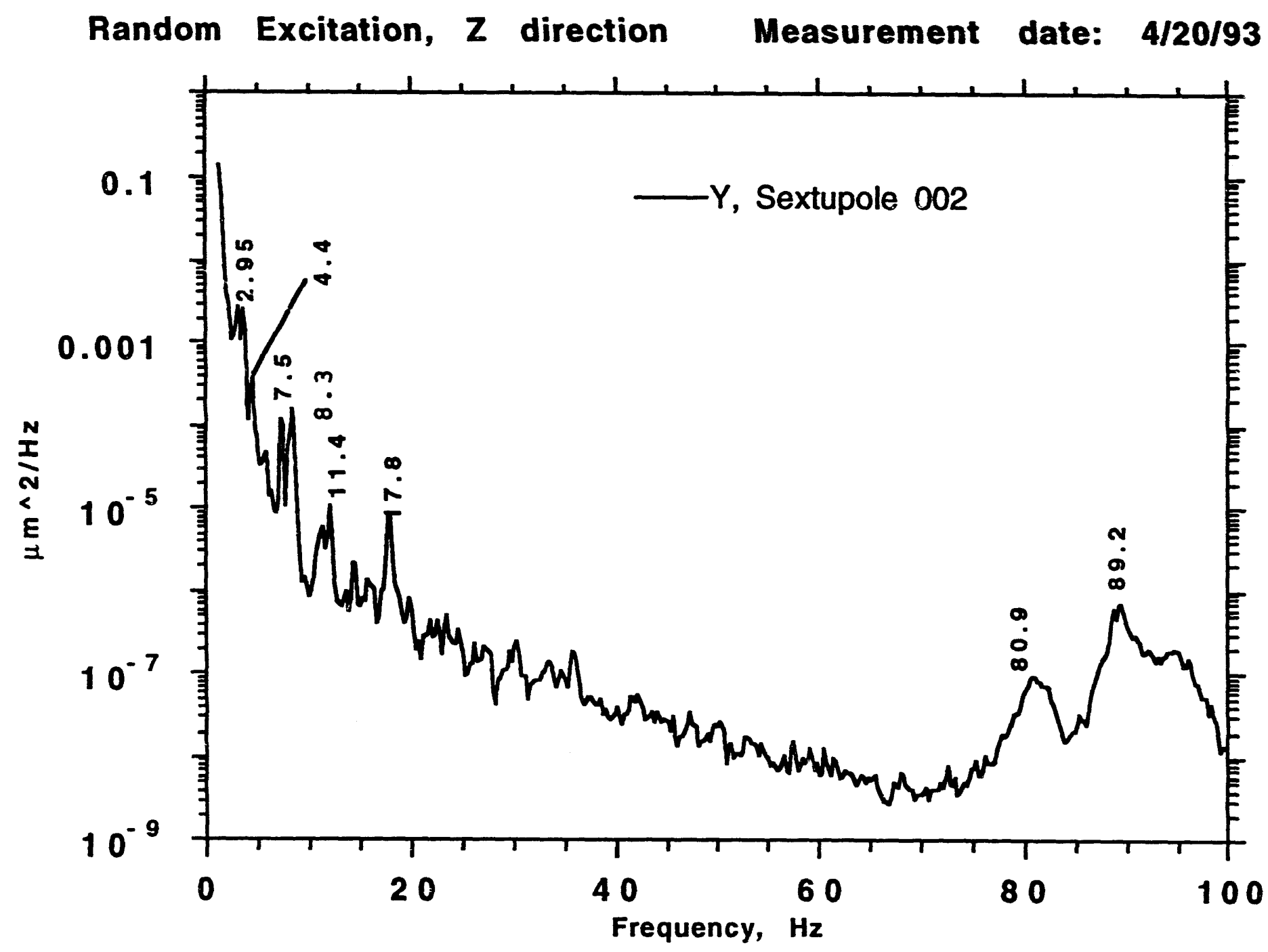




\section{SYNCHROTRON GIRDER RESPONSE \\ Girder \# B2C61}

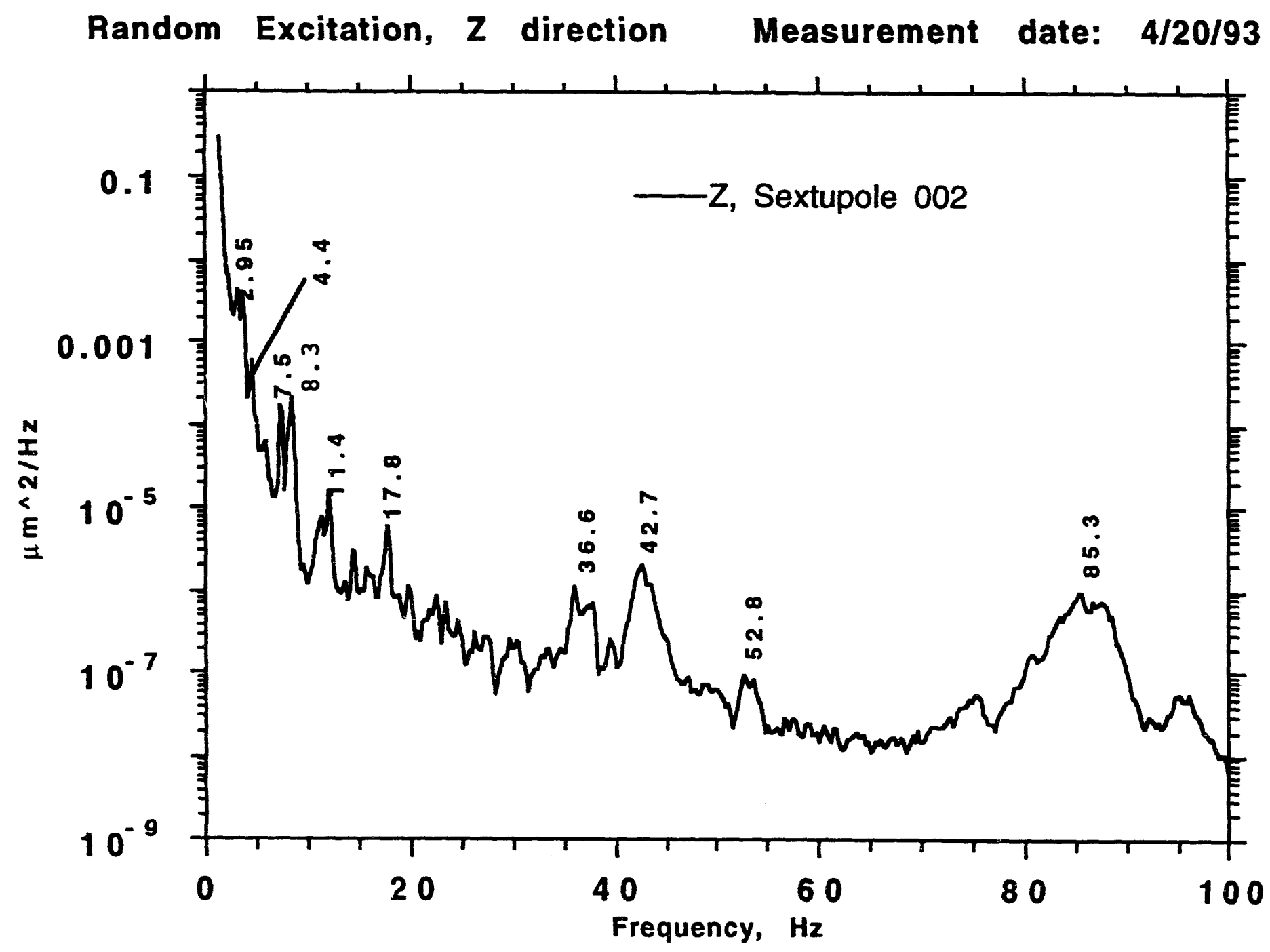




\section{SYNCHROTRON GIRDER RESPONSE}

Girder \# B2C61

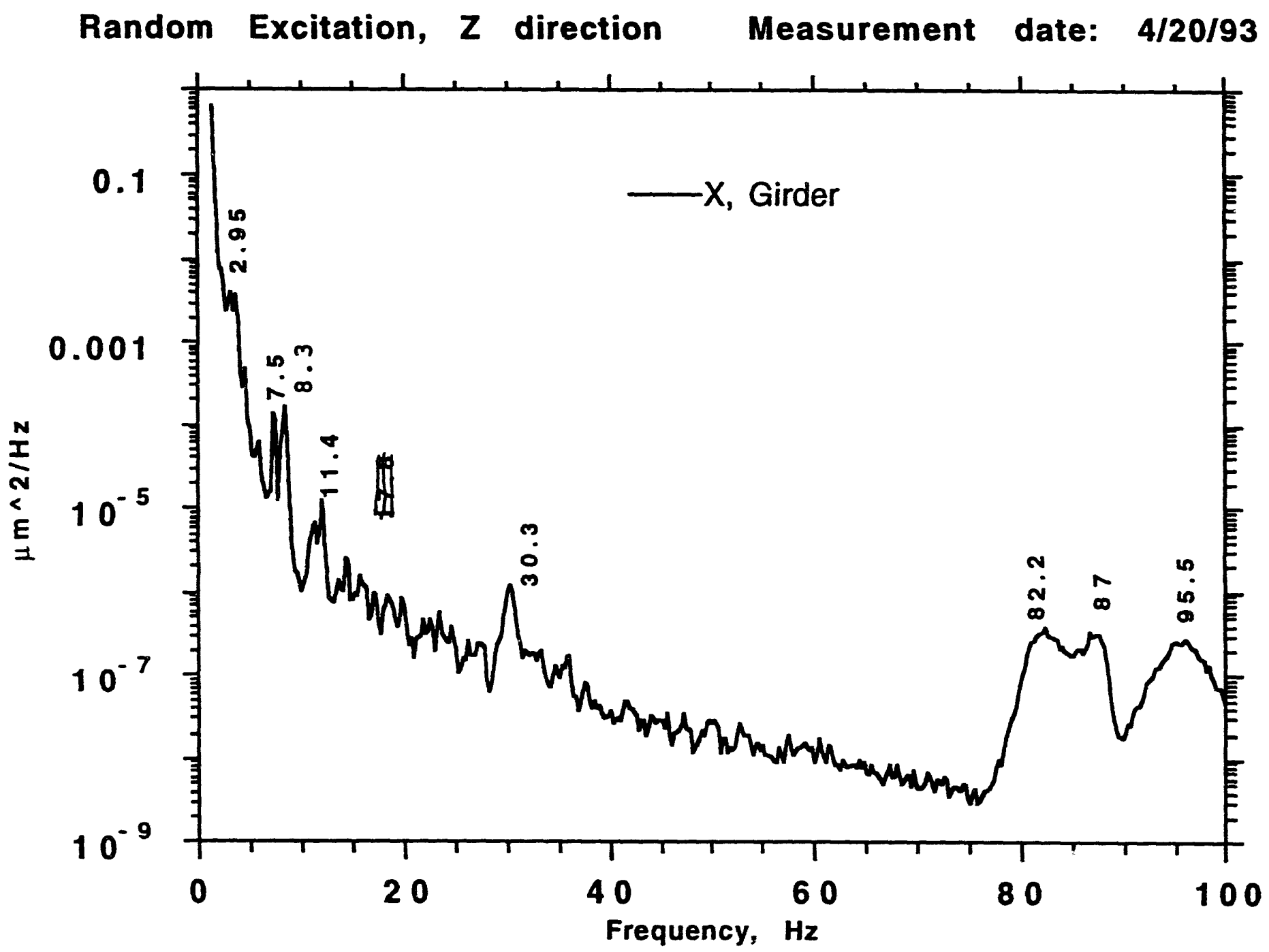




\section{SYNCHROTRON GIRDER RESPONSE}

Girder \# B2C61

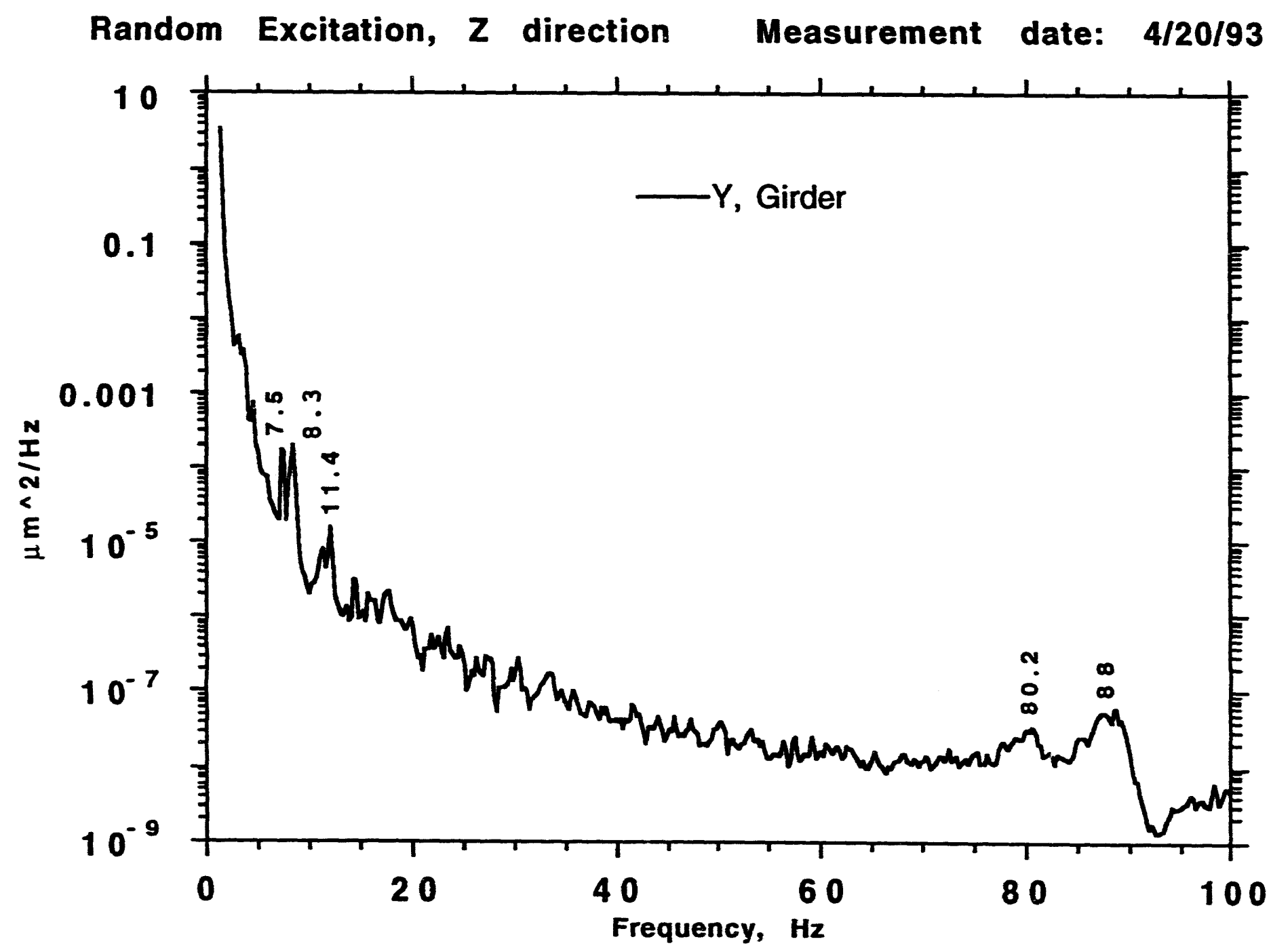




\section{SYNCHROTRON GIRDER RESPONSE}

Girder \# B2C61

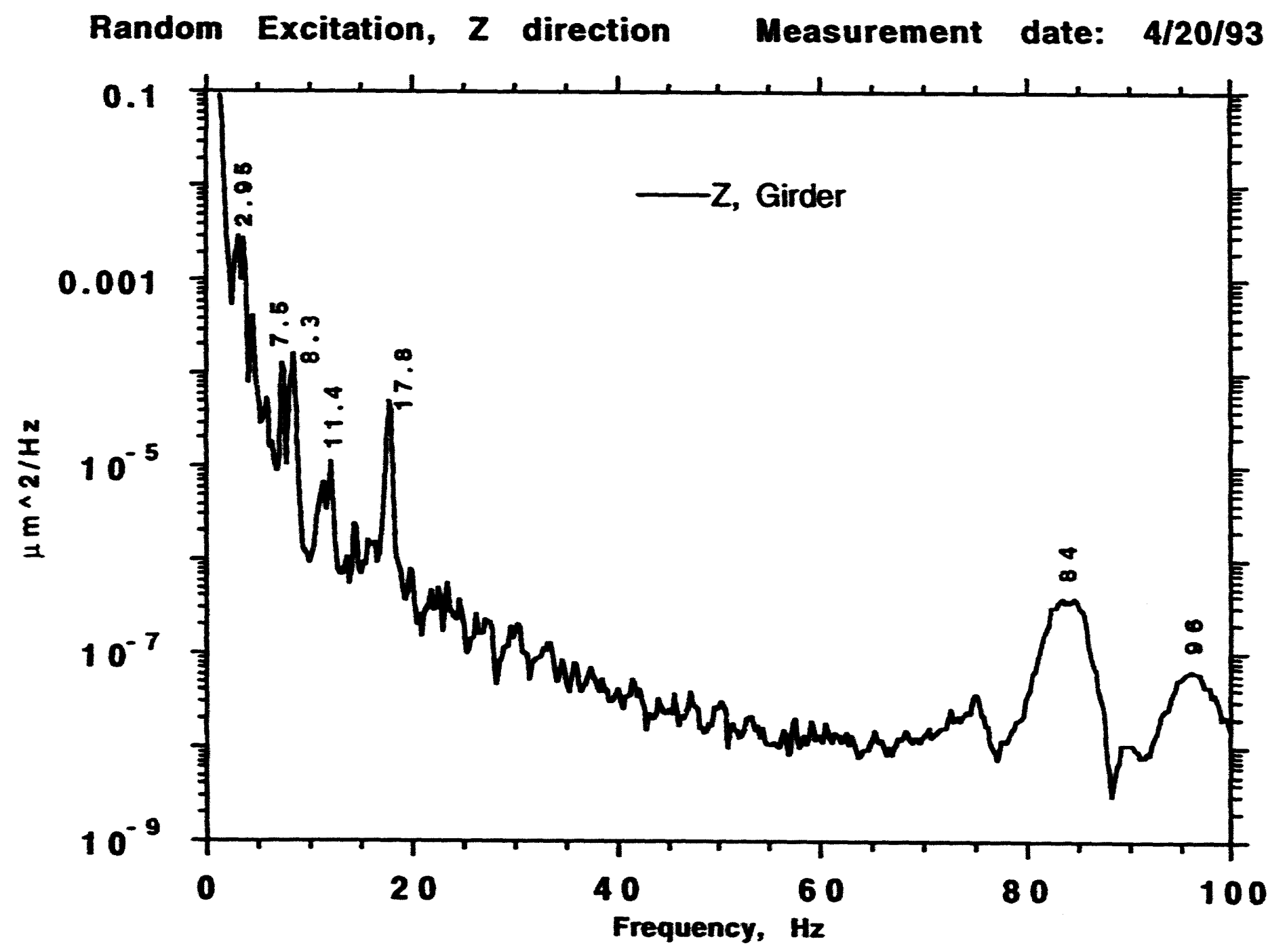




\section{SYNCHROTRON GIRDER RESPONSE}

Girder $82 \mathrm{C61}$

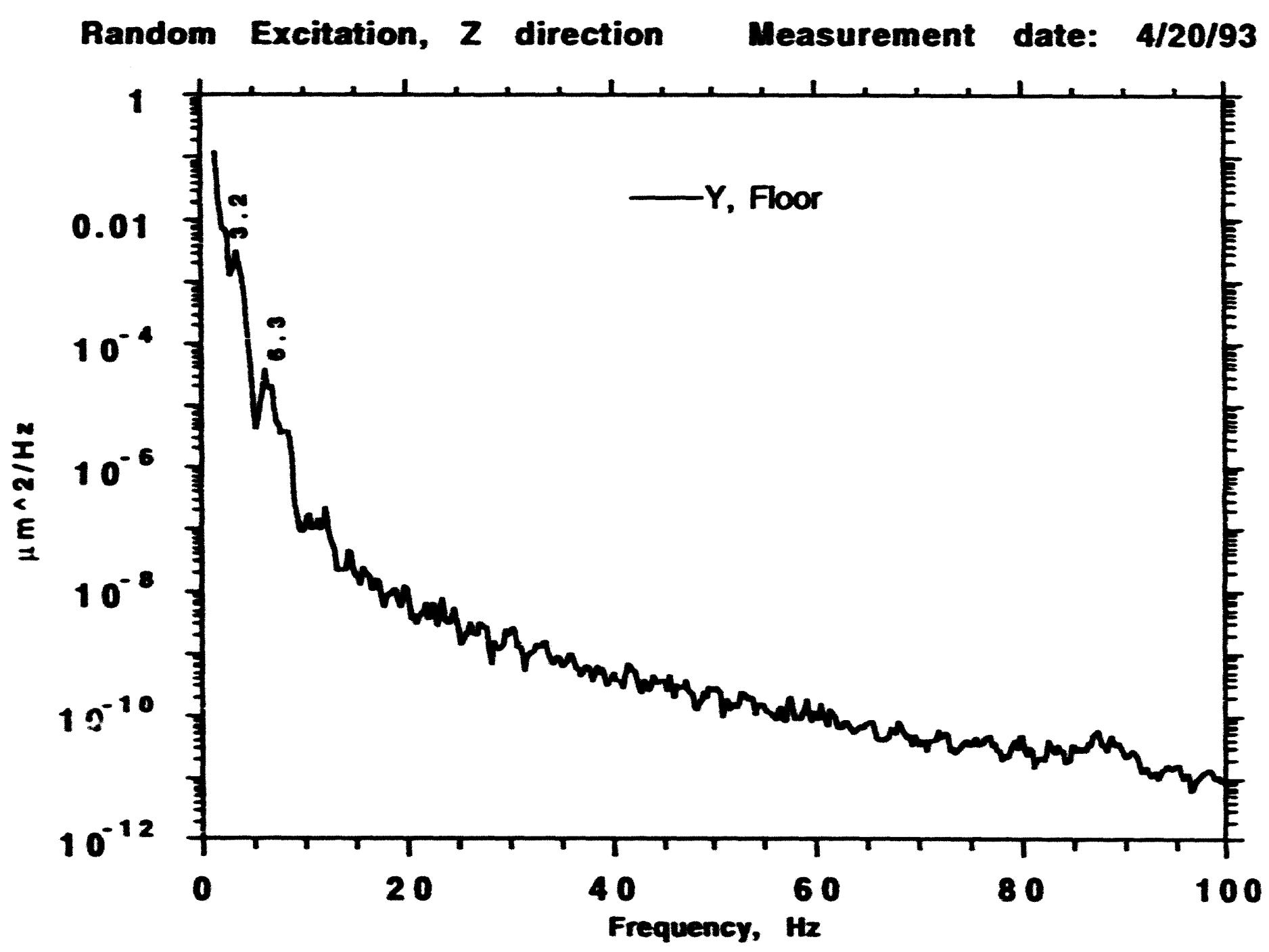


SWCHROTRON GARDER RESPONSE

Girder B2C61

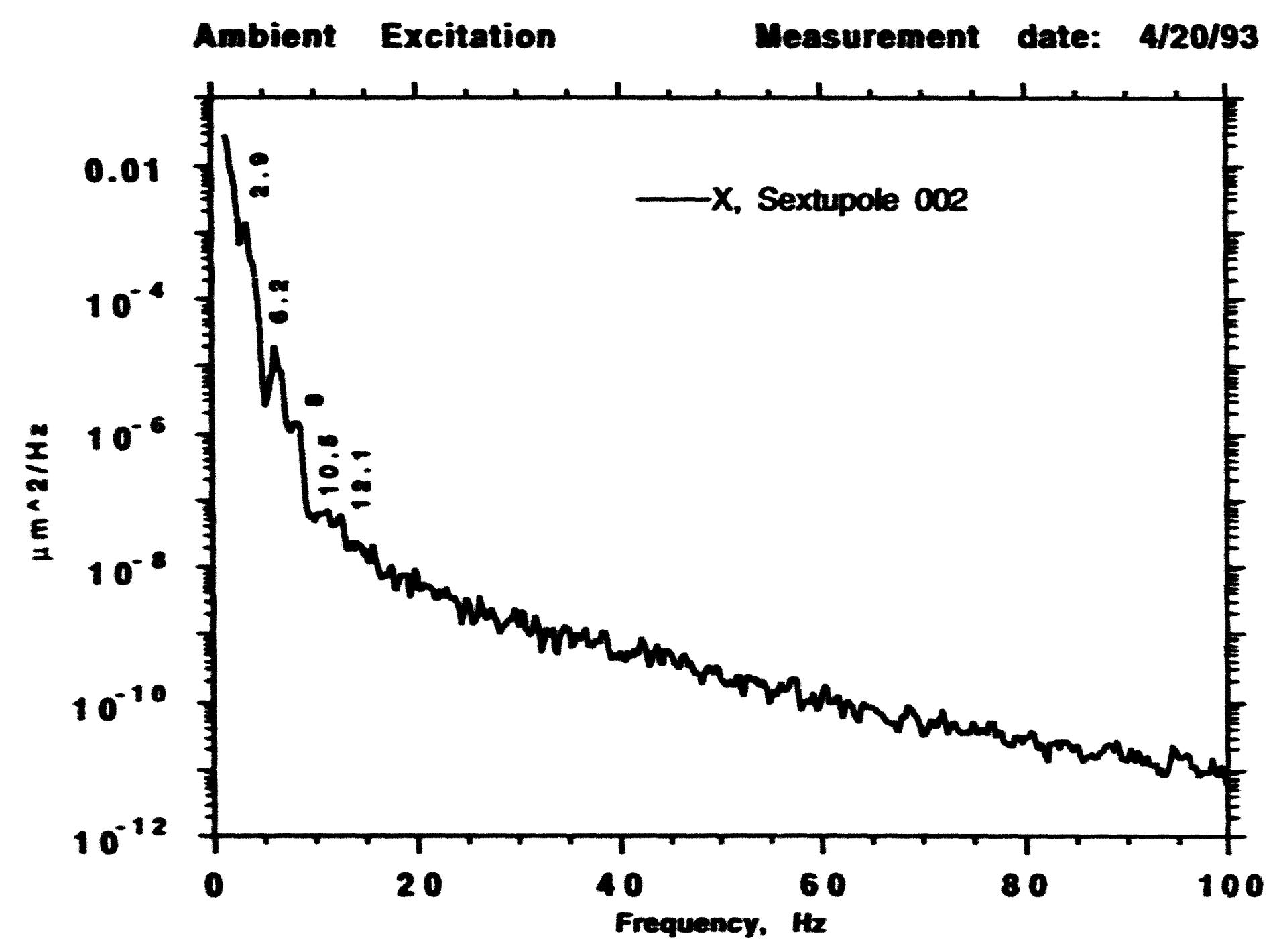

I 
SYNCHROTRON GIRDER RESPONSE

Girder B2C61

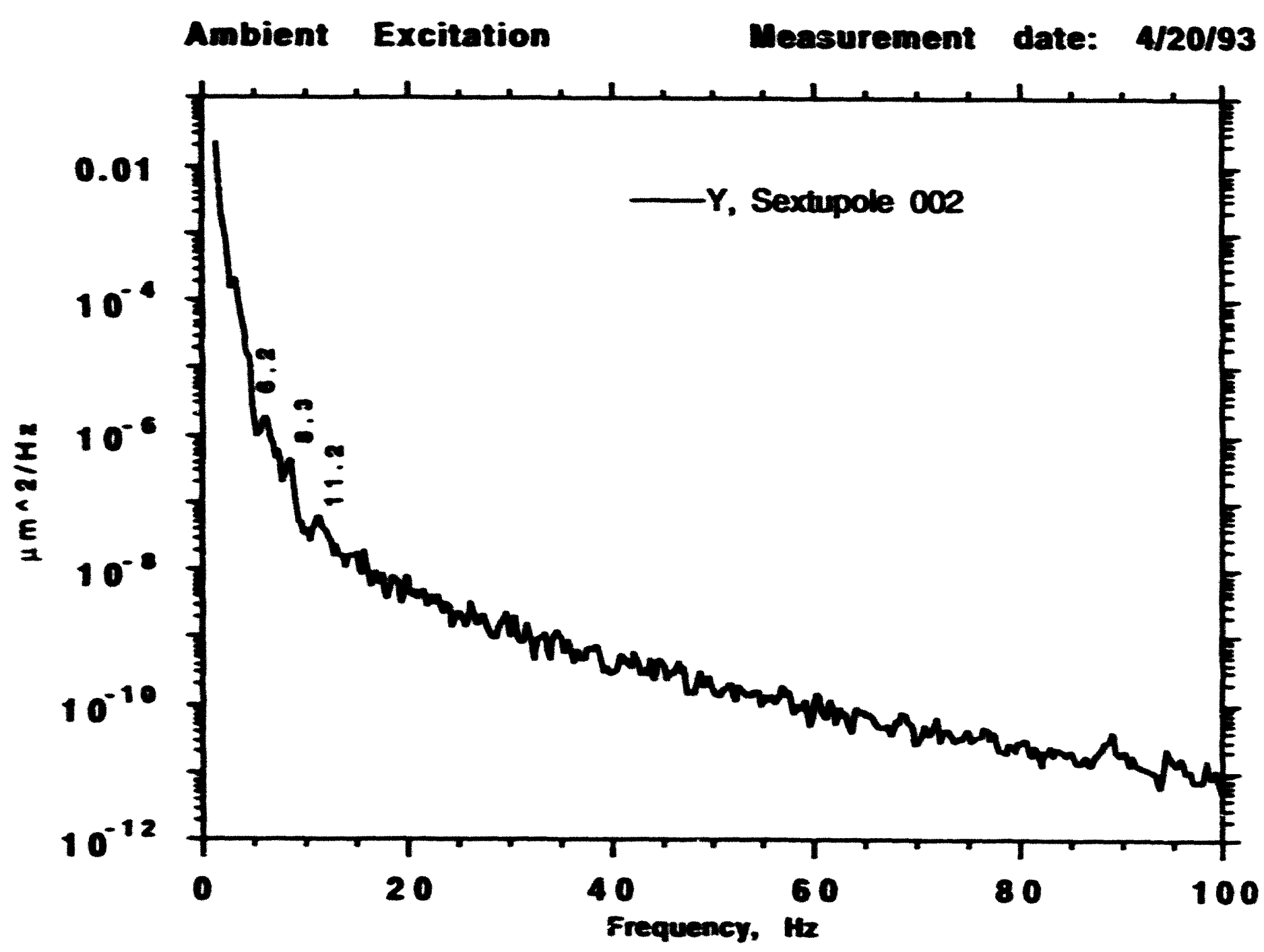

足 
SWNCHROTRON GIRDER RESPONSE

Girder B2C61

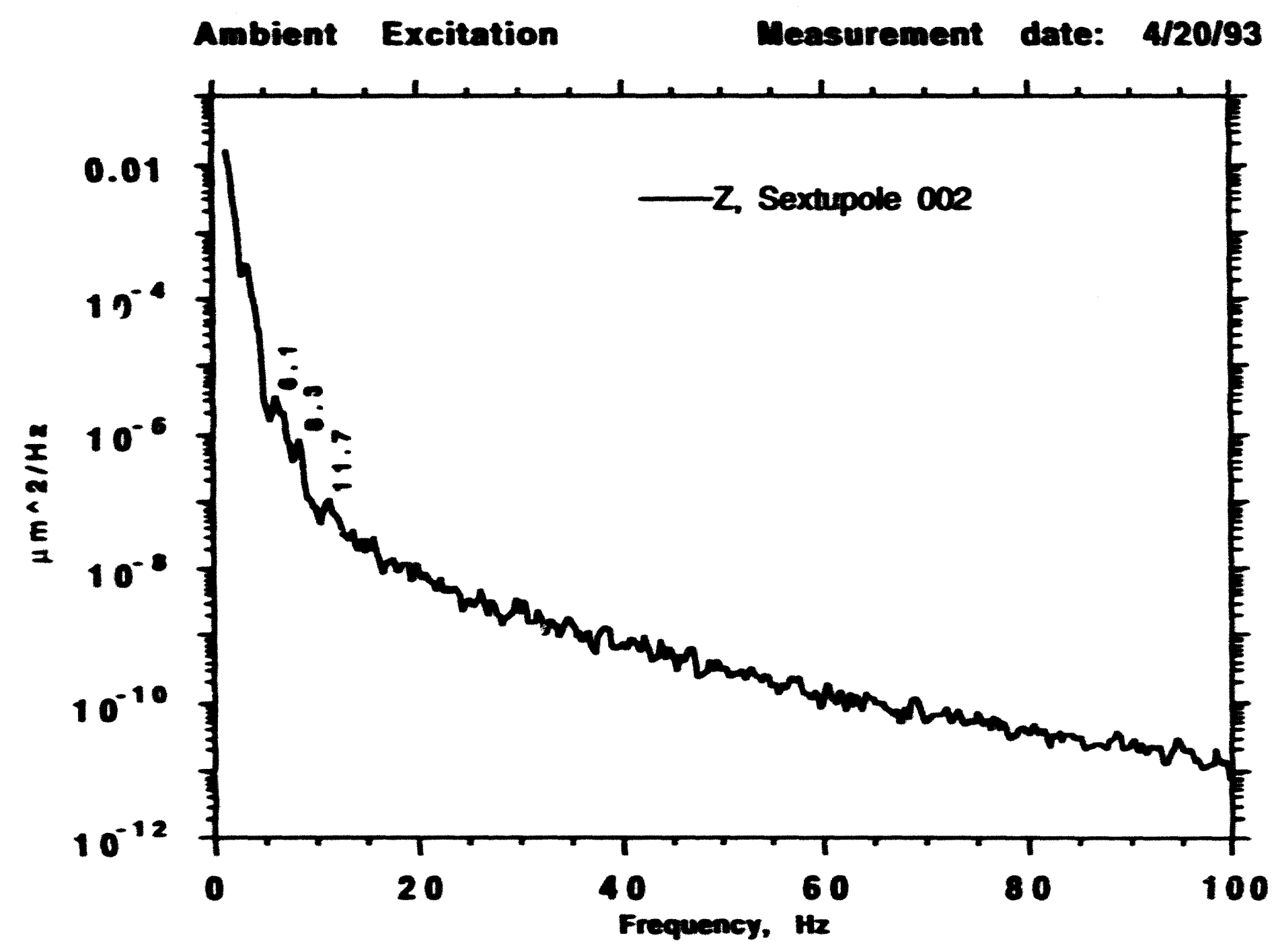


SYNCHROTRON GIRDER RESPONSE

Girder B2C61

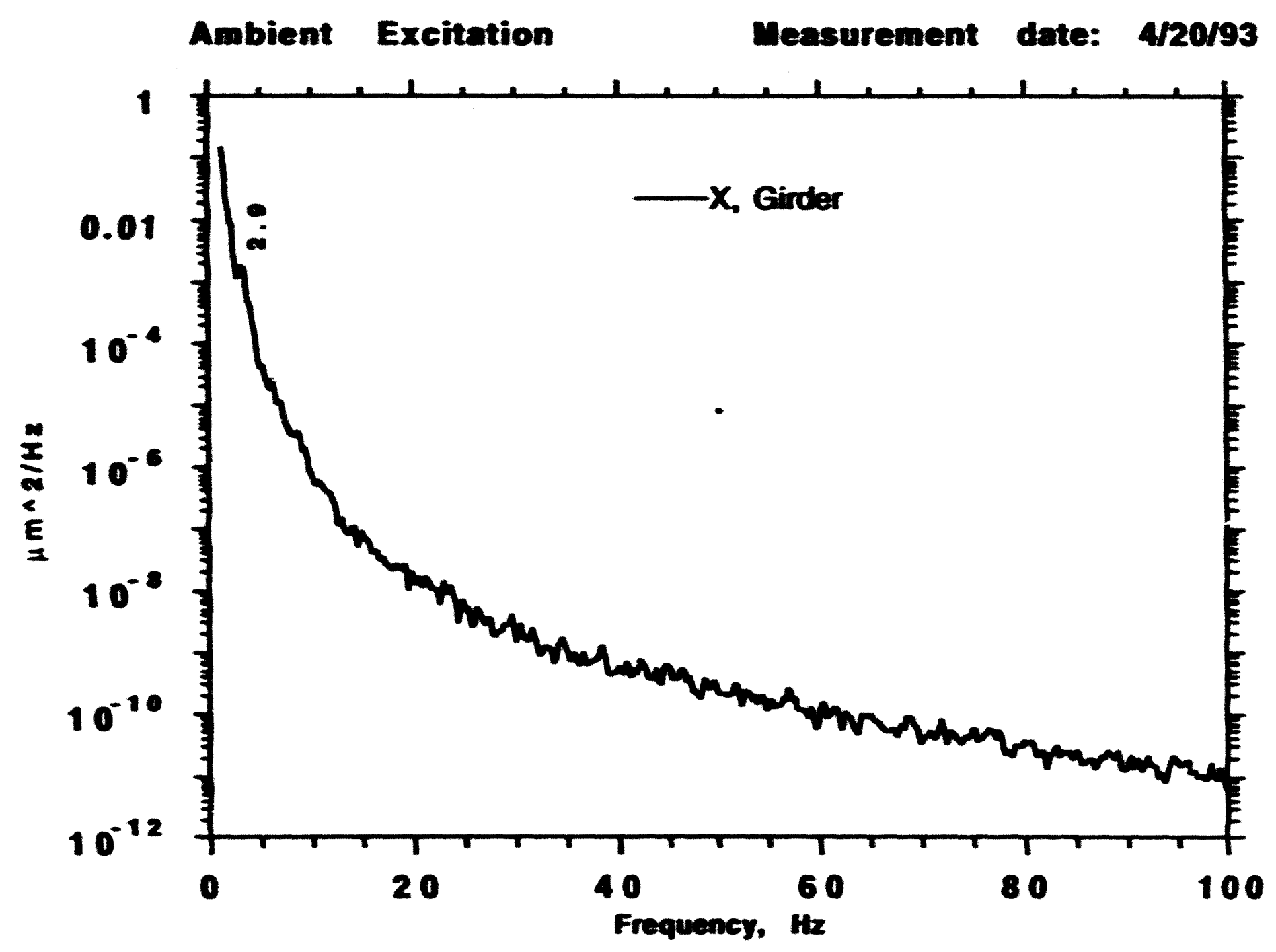

ป 
SYNCHROTRON GIRDER RESPONSE

Girder B2C61

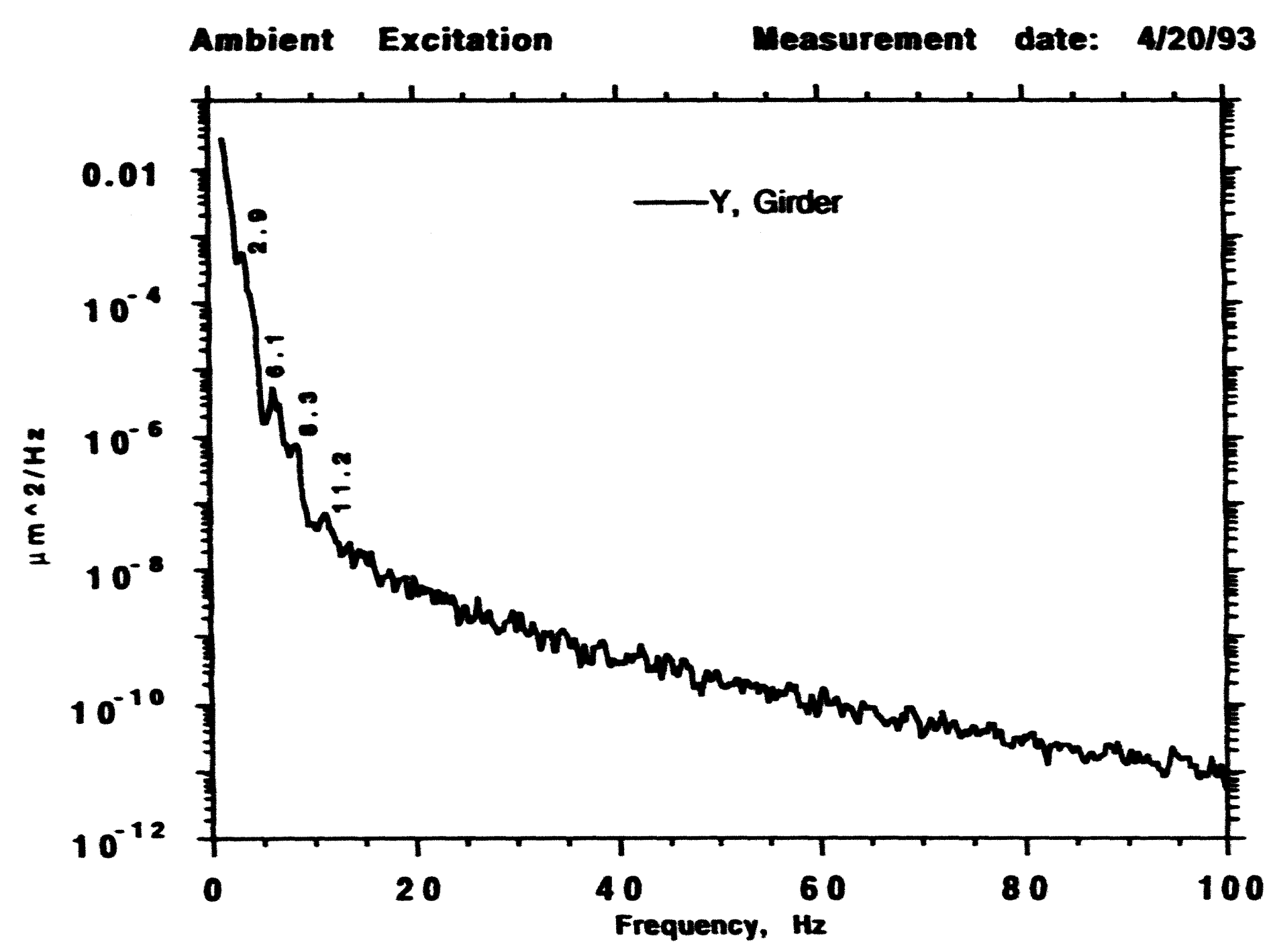




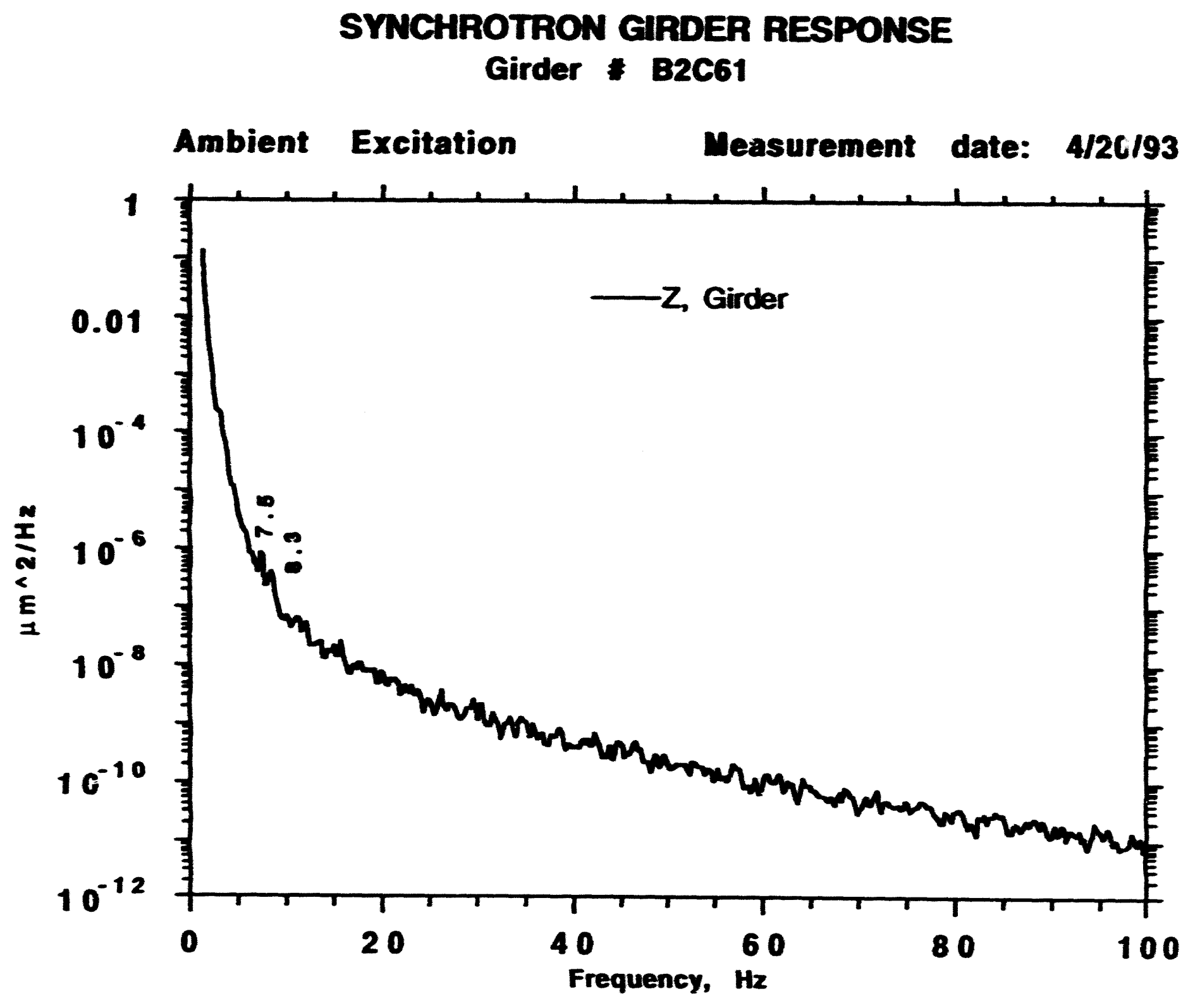

0
1
1 
SYNCHROTRON GIRDER RESPONSE

Girder \# B2C61

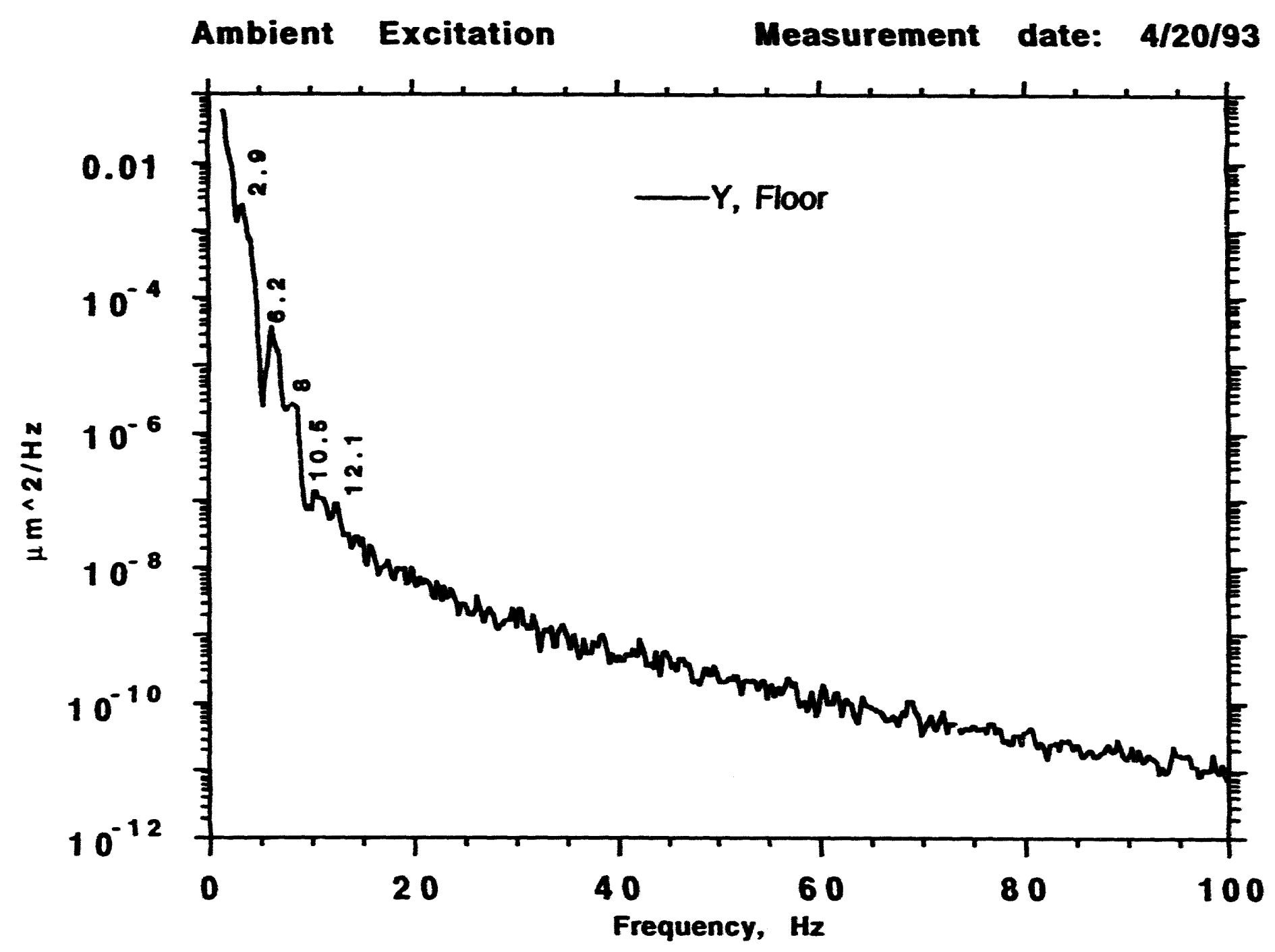


Appendix E: Floor/girder response 
E-1

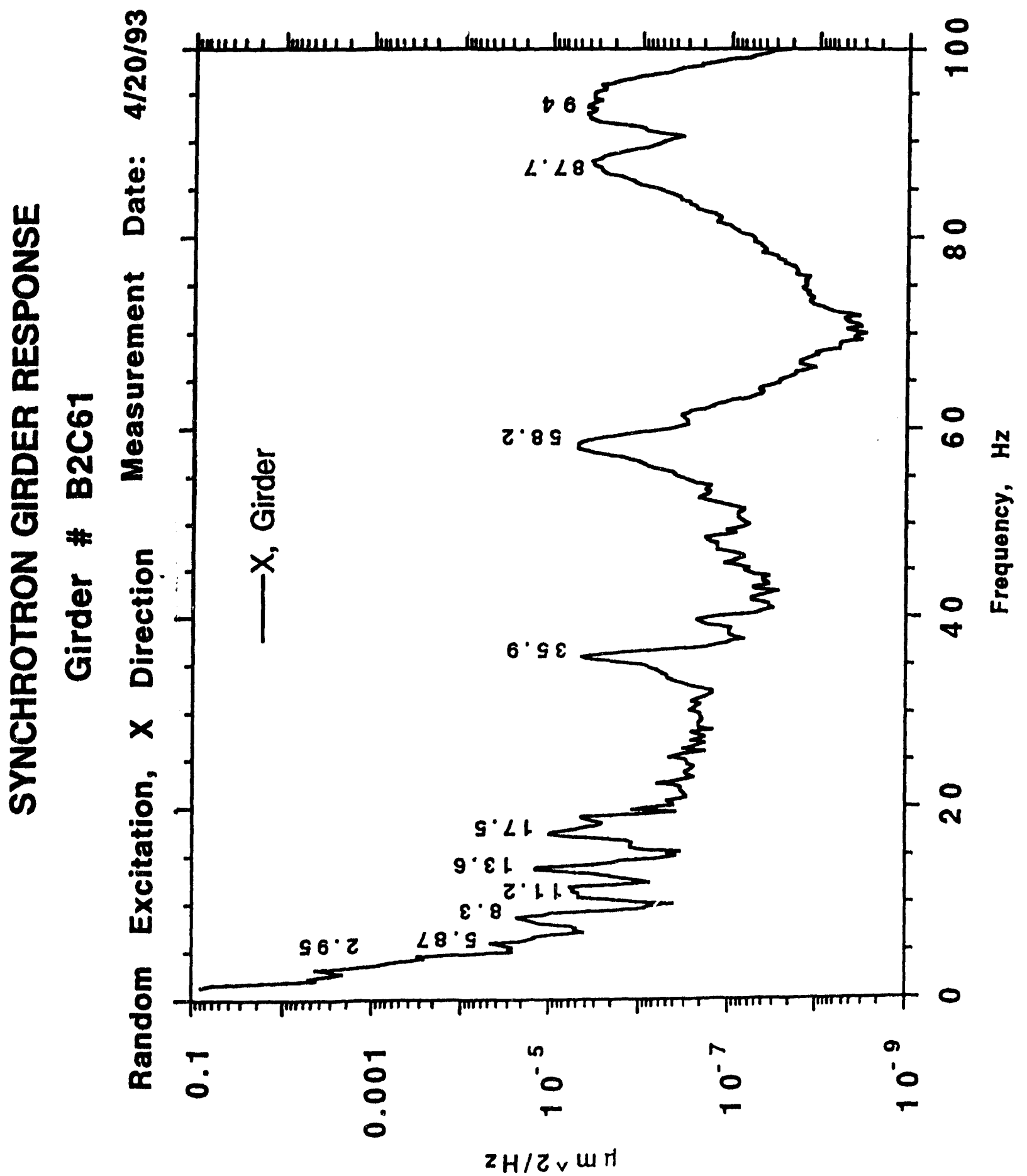




\section{SYNCHROTRON GIRDER RESPONSE}

Girder \# B2C61

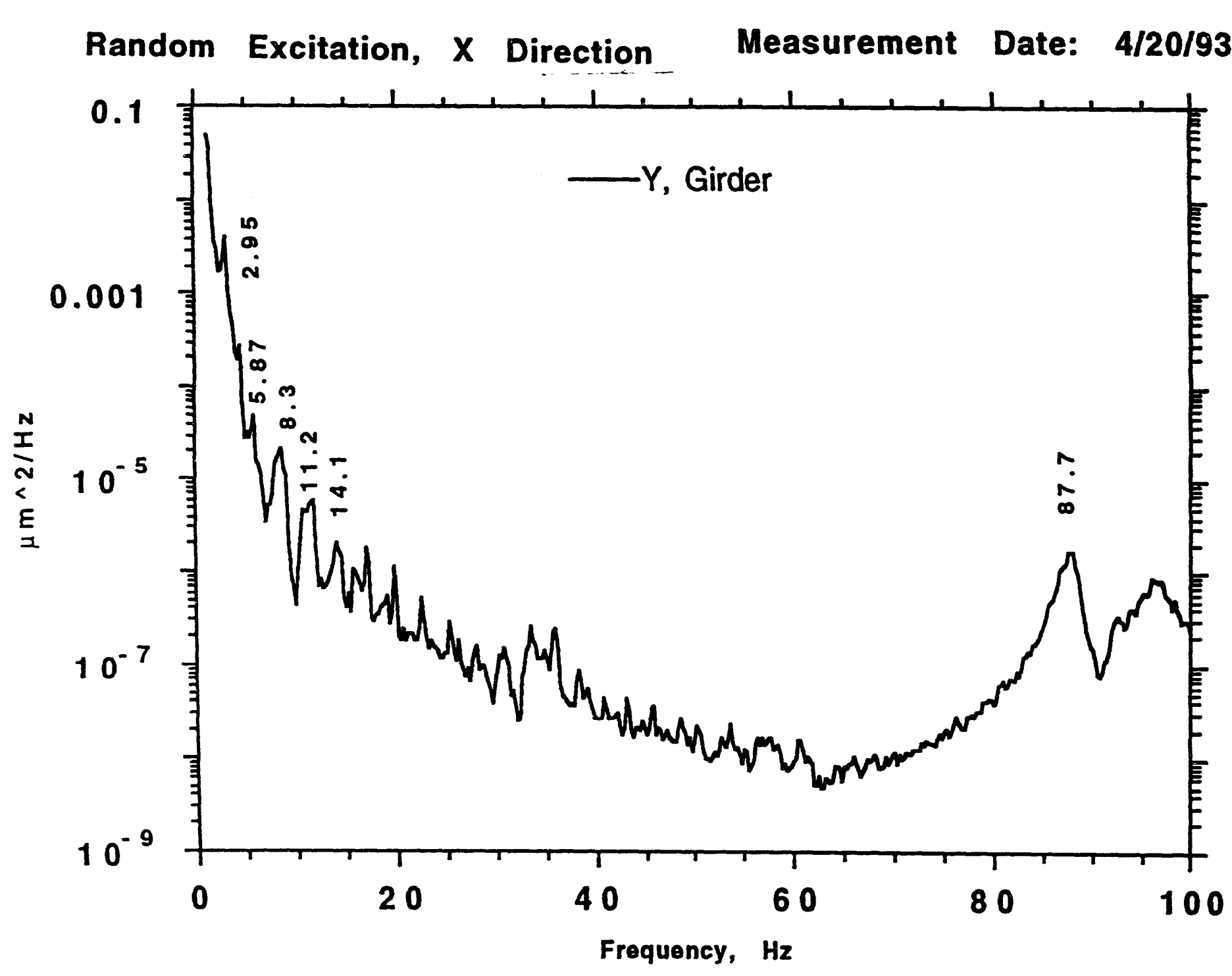




\section{SYNCHROTRON GIRDER RESPONSE}

Girder \# B2C61

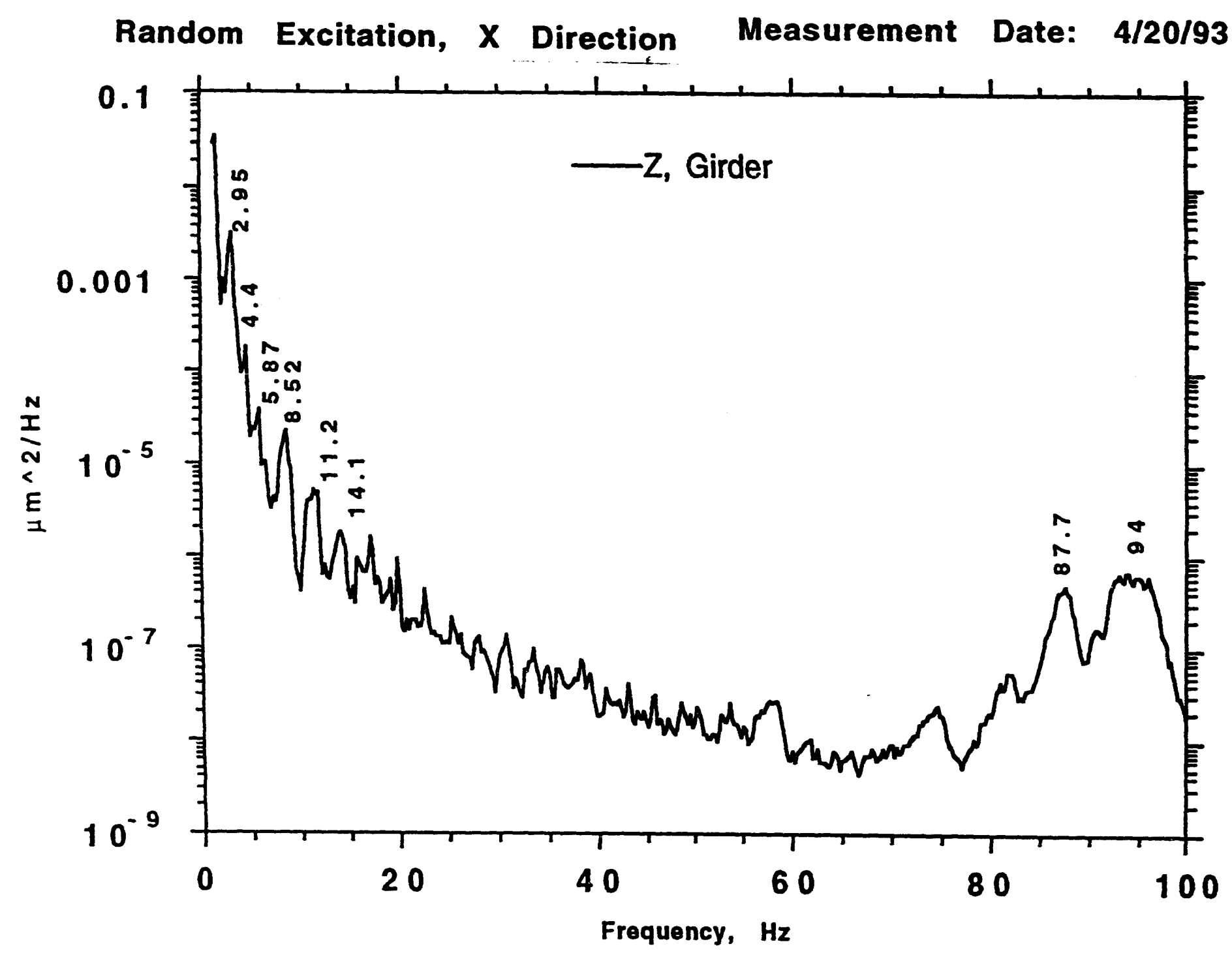




\section{SYNCHROTRON GIRDER RESPONSE}

Girder \# B2C61

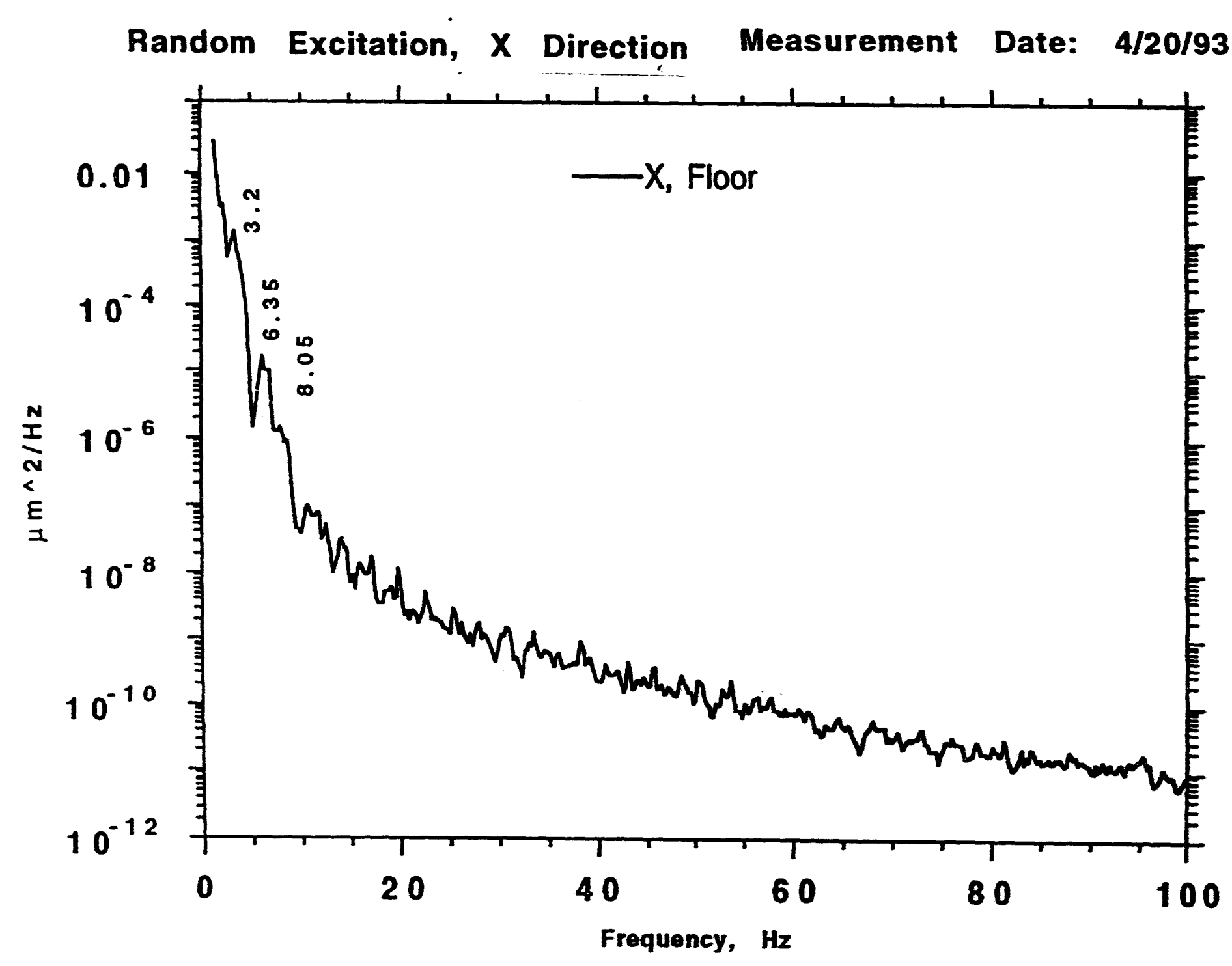




\section{SYNCHROTRON GIRDER RESPONSE}

Girder \# B2C61

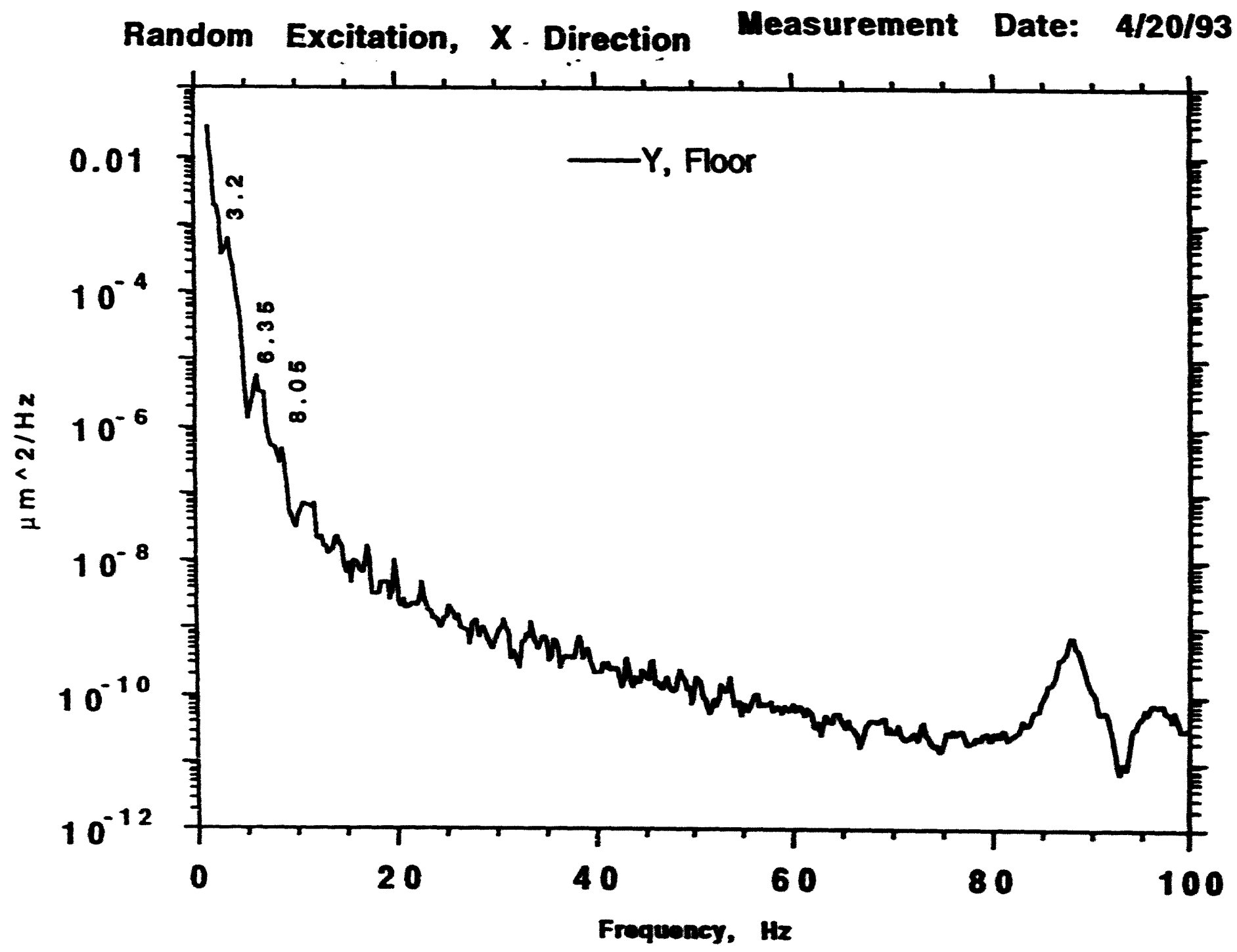




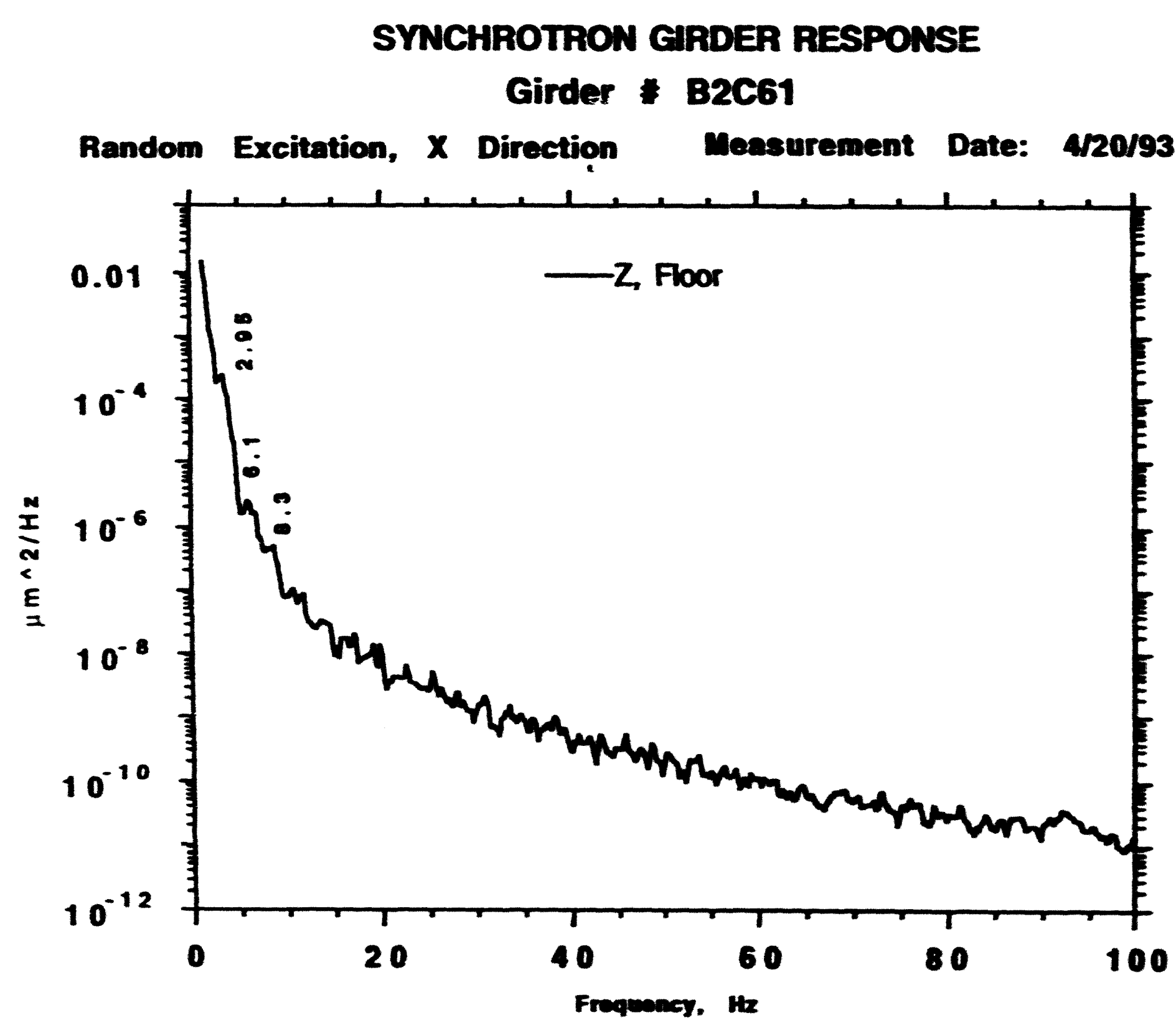

5 
SYNCHROTRON GRDER RESPOUSE

Girder B2C61

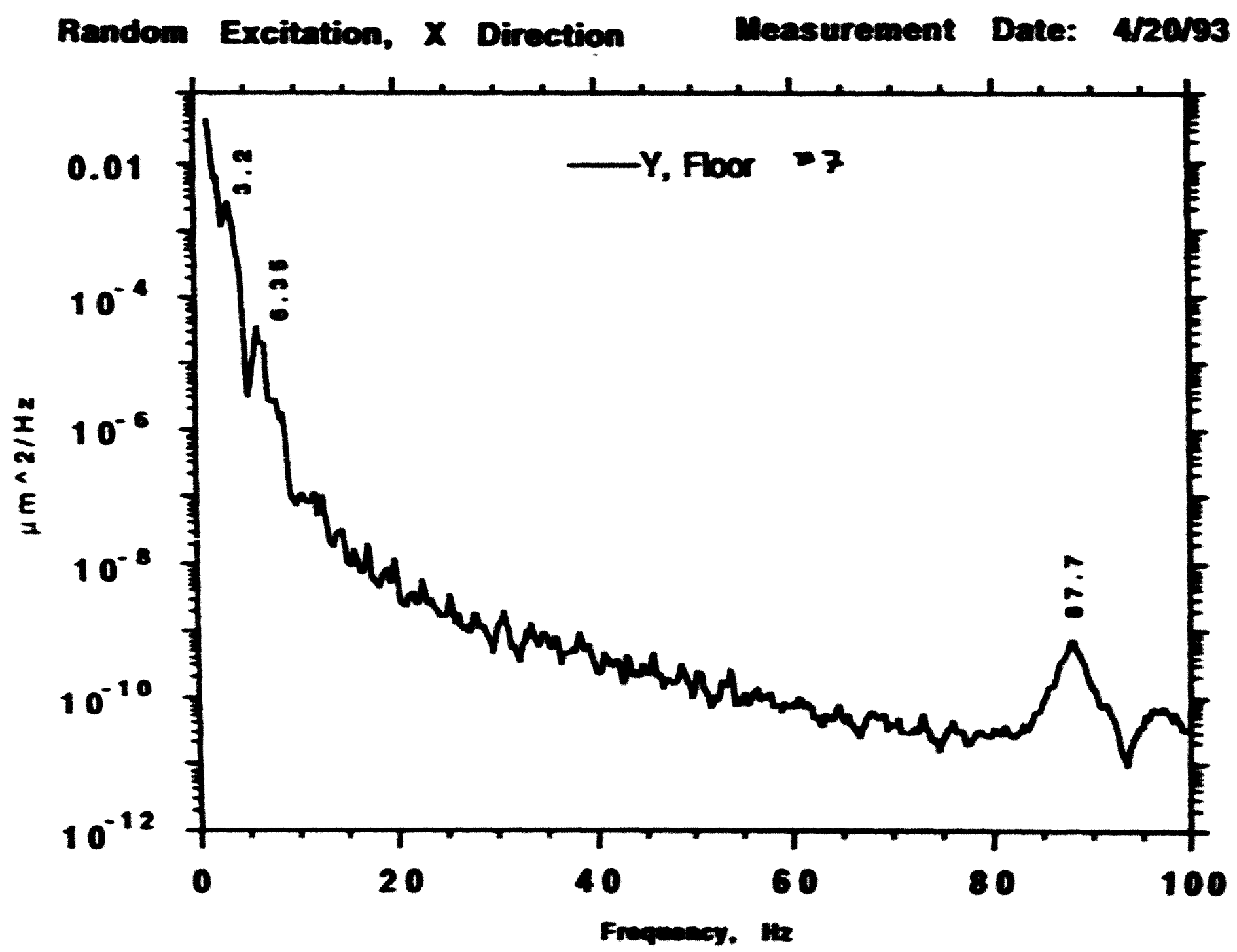



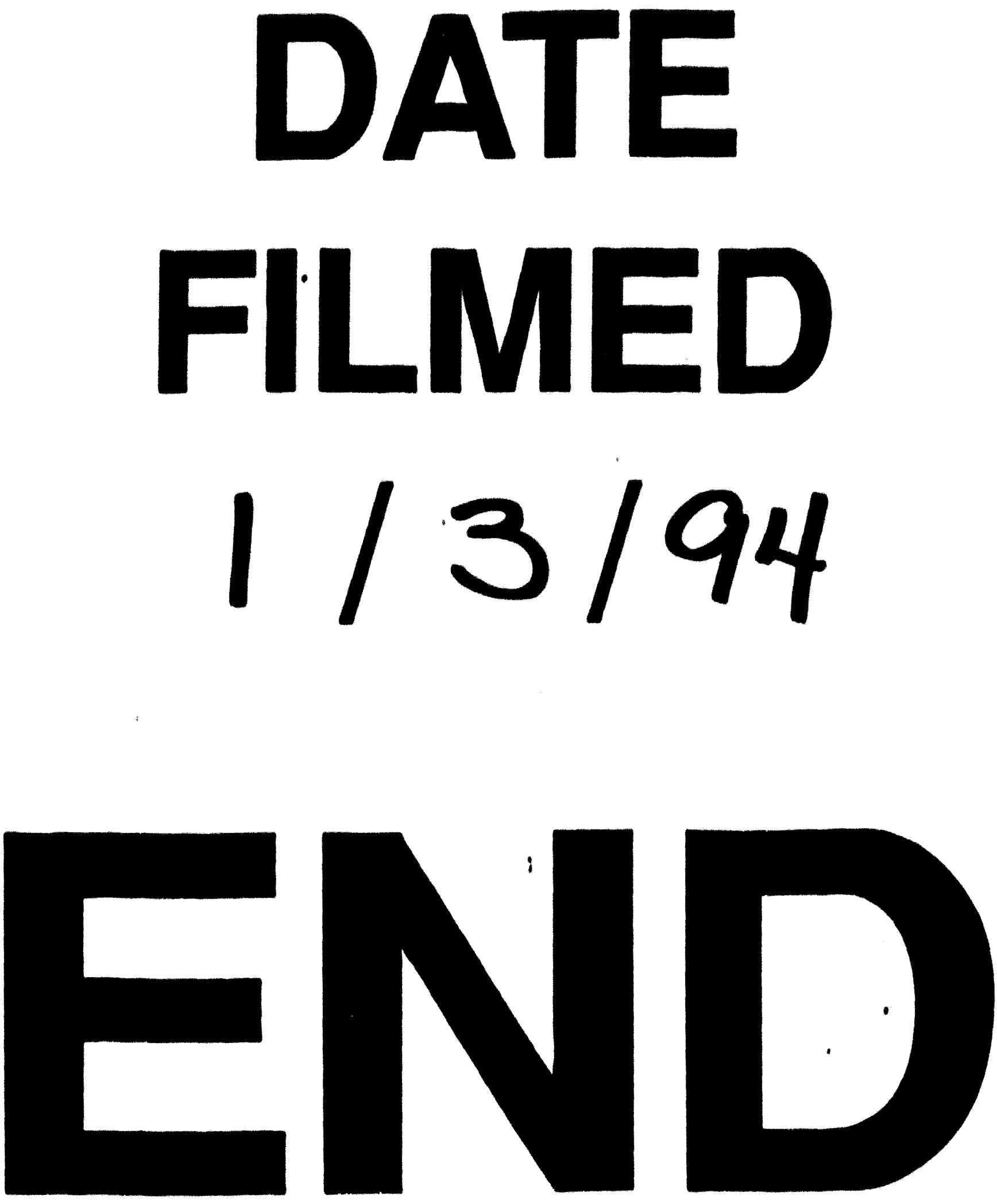

$\mid$ 